\title{
Mechanism of Germ Cell Specification in Early Zebrafish Embryogenesis
}

\author{
Doctoral Thesis
}

Dissertation for the award of the degree

"Doctor rerum naturalium (Dr. rer. nat)"

in the GGNB program: Genes and Development

at the Georg August University Göttingen

Faculty of Biology

Submitted by

Roshan Priyarangana Perera

Born in Sri Lanka

Göttingen, June 2020 


\section{Members of the Thesis Committee}

\section{Supervisor}

Dr. (PD). Roland Dosch (Reviewer)

Institute for Human Genetics, Georg August University Göttingen

\section{Second member of the thesis committee}

Professor. Markus T.Bohnsack (Reviewer)

Institute for Molecular Biology, Georg August University Göttingen

Third member of the thesis committee

Dr. Achim Dickmanns

Department for Structural Biology, Georg August University Göttingen

\section{Examination board members}

Professor. Gregor Bucher

Department of Developmental Biology, Georg August University Göttingen

Dr. Nico Posnien

Department of Developmental Biology, Georg August University Göttingen

Dr. Ufuk Günesdogan

Department of Developmental Biology, Georg August University Göttingen

Date of Oral examination: 


\section{Affidavit}

Herewith I declare that I prepared the PhD thesis "Mechanism of Germ Cell Specification in Early Zebrafish Embryogenesis" on my own and with no other sources and aids than quoted.

04.06.2020

Roshan Perera 


\section{Acknowledgement}

I wish to express my sincere gratitude to my supervisor, Dr. Roland Dosch, the one who convincingly guided and encouraged me to be professional and do the right things even when the road got more tough than I expected.

Besides my supervisor, I would like to thank my thesis committee: Prof. Markus Bohnsack and Dr. Achim dickmann, for their encouragement, insightful comments, and constructive questions.

I thank my fellow labmates in the Dosch Lab: Dr. Pritesh Krishnakumar, Dr. Alexander Goloborodko, Nadia Rostam, Hazem Khalifa and Vranda Garg for nice discussions, arguments, for the sleepless nights we were working together in the lab, and for all the fun we have had in the last four years.

Words cannot express how grateful I am for you Alaa Shaikhqasem. Thank you for your help and useful advice! I am incredibly fortunate to have a friend like you. I could not have done my Ph.D. without you. Also, I would like to thank Piotr Neumann, Tim Garbers, and Florian Hamann for your support for my studies.

I specially thank my friend Leoni Witte: You are a wonderful friend. You inspired me during a difficult time when I needed words of encouragement, and I appreciate your, kindness, support, and generosity shared with me during the last four years. Also, my deep appreciation goes to my friends Dolma Choezom, Pradhipa Karuna, and Esther Essel who helped me in numerous ways during various stages of my Ph.D.

I am indebted to all my rotation students, Lukas Amann, Aishwarya Tagat, Indrani Mukarji, and Florian Fink for their valuable contribution to my research project.

My thanks also go out to Gudrun Krach, Mona Honemann, and Katja Ditter who were always so helpful and provided me with their kind assistance. In addition, I thank to Dr. Julia Gross allowing me to do cell culture experiments.

This work would not have been possible without financial support from Deutscher Akademischer Austausch Dienst/German Academic Exchange Service. Therefore, I express my heartfelt thanks to the for funding thought out my Ph.D. Special thanks go to Göttingen Graduate Center for Neurosciences, Biophysics, and Molecular Biosciences (GGNB)for their enormous support during my Ph.D.

I wish to acknowledge the support and great love of my Mother, Father, and other family members. They kept me going on and this work would not have been realized without their support. 


\section{List of Abbreviations}

${ }^{\circ} \mathrm{C}$

A

aa

amol

bp

BSA

C

C. elegans

cDNA

CMV

Co-IP

C-terminus

DAZ

$\mathrm{dH}_{2} \mathrm{O}$

$D m$

DNA

dNTP

dpf

E. coli

EDTA

eGFP

EGTA

et al.

fw

g

G
Degrees Celsius

Adenine

Amino acids

Attomolar

Base pairs

Bovine serum albumin

Cytosine

Caenorhabditis elegans

Complementary DNA

Cytomegalovirus

Co-immunoprecipitation

Carboxy-terminus

Deleted in azoospermia

Distilled water

Drosophila melanogaster

Deoxyribonucleic acid

Deoxynucleotide triphosphate

Days post fertilization

Escherichia coli

Ethylenediaminetetraacetic acid

Enhanced green fluorescent protein

Ethylene glycol tetraacetic acid

Et alii

Forward

Gram

Guanine 


\begin{tabular}{|c|c|}
\hline GFP & Green fluorescent protein \\
\hline h & Hour \\
\hline HMM & Hidden Markov models \\
\hline hpf & Hours post fertilization \\
\hline IP & Immunoprecipitation \\
\hline $\mathrm{Kb}$ & Kilo base pairs \\
\hline kDa & Kilodalton \\
\hline LB & Lysogeny broth \\
\hline M & Molar \\
\hline $\mathrm{mg}$ & Milligram \\
\hline $\min$ & Minute \\
\hline miRNA & Micro RNA \\
\hline $\mathrm{ml}$ & Millilitre \\
\hline $\mathrm{mM}$ & Millimolar \\
\hline mRNA & Messenger RNA \\
\hline $\mathrm{n}$ & Number \\
\hline ng & Nanogram \\
\hline $\mathrm{nl}$ & Nanolitre \\
\hline N-terminus & Amino-terminus \\
\hline ORF & Open reading frame \\
\hline PAGE & Polyacrylamide gel electrophoresis \\
\hline PBS & Phosphate-buffered saline \\
\hline PBT & Phosphate-buffered saline Triton X-100 \\
\hline PCR & Polymerase chain reaction \\
\hline pg & Picogram \\
\hline PGC & Primordial germ cell \\
\hline $\mathrm{pH}$ & Potentium hydrogenium \\
\hline piRNA & Piwi-interacting RNA \\
\hline
\end{tabular}




\begin{tabular}{|c|c|}
\hline RNA & Ribonucleic acid \\
\hline RNase & Ribonuclease \\
\hline RNP & Ribonucleo-protein granule \\
\hline rpm & Rounds per minute \\
\hline RRM & RNA recognition motif \\
\hline RT-qPCR & Reverse transcription quantitative real-time PCR \\
\hline rv & Reverse \\
\hline S & Second \\
\hline SDS & Sodium dodecyl sulfate \\
\hline $\mathrm{SH} 3$ & SRC Homology 3 \\
\hline $\mathrm{T}$ & Thymine \\
\hline TAE & Tris-Acetate-EDTA \\
\hline Taq & Thermus aquaticus \\
\hline TBE & Tris-Borate-EDTA \\
\hline Tris & Tris(hydroxymethyl)aminomethane \\
\hline$U$ & Units \\
\hline UAS & Upstream activation sequence \\
\hline UTR & Untranslated region \\
\hline UV & Ultraviolet \\
\hline V & Volt \\
\hline Zf & Zebrafish \\
\hline$\mu g$ & Microgram \\
\hline$\mu l$ & Microlitre \\
\hline$\mu \mathrm{m}$ & Micrometer \\
\hline$\mu \mathrm{M}$ & Micromolar \\
\hline
\end{tabular}




\section{Abstract}

Reproduction is an essential feature of life that ensures the perpetuation and survival in many multicellular organisms while passing the genetic information to the next generation. The zebrafish germline is specified during early embryogenesis by maternal RNP granules collectively called germ plasm. It has been discovered that the zebrafish bucky ball gene, which is a key gene responsible for germ plasm formation and encodes a novel vertebratespecific protein with unknown biochemical function. Overexpression experiments revealed that Buc induces ectopic primordial germ cells (PGCs) in vivo. So far, only the Oskar protein in Drosophila shows equivalent activities. Remarkably, we recently revealed that Oskar induces ectopic PGCs in zebrafish, albeit Oskar does not share sequence homology with Buc. Moreover, zebrafish Vasa is also sufficient to induce PGCs in vivo suggesting that Buc and Vasa act in the same molecular process involving in germ cell specification. This conserved activity between Oskar and Buc suggests that both proteins share a similar biochemical interactome, whose molecular identity is unknown.

In this study, I characterize the interaction of Buc and the RNA helicase Vasa investigating their binding motifs. I identified a peptide encoding amino acids 372-394 in Buc as Vasa Binding Motif (VBM) and a peptide encoding amino acids 600-625 in Vasa as Buc Binding Motif (BBM) using bimolecular fluorescence complementation (BiFC). Further, I show that the Buc-VBM is highly conserved in vertebrates. Moreover, Buc and Vasa directly interact in vitro and independent of RNA. The Buc-VBM encodes an intrinsically disordered peptide, but CD spectroscopy reveals that a crowding agent induces formation of an $\alpha$-helix. Fascinatingly, I discovered that Buc activates Vasa ATPase activity annotating the first biochemical function of Buc as a helicase activator. Collectively, these results propose a model in which the activity of Vasa helicase is a central regulator of PGC formation, which is tightly controlled by the germ plasm organizer Buc. 


\section{Table of Contents}

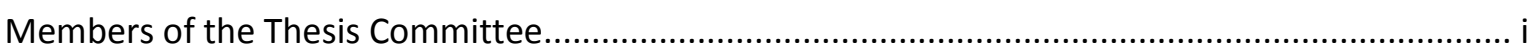

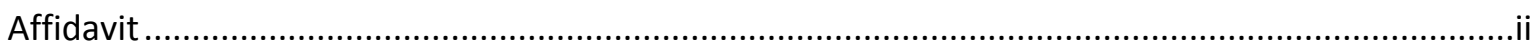

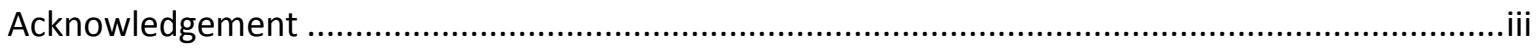

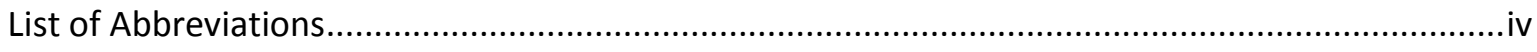

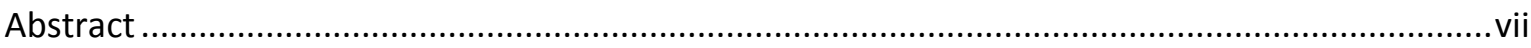

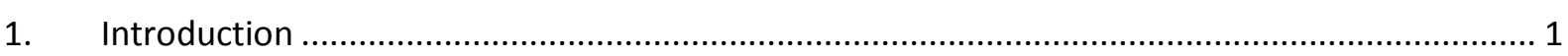

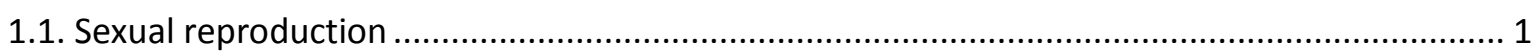

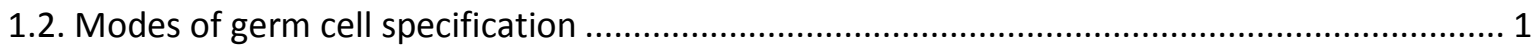

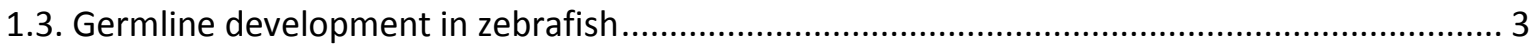

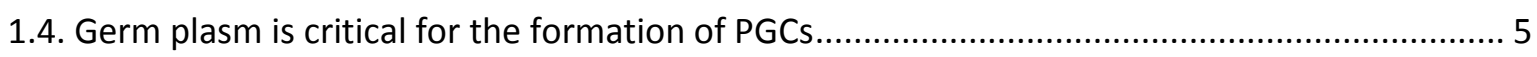

1.5. Roles of known germ plasm components during germ cell specification................................... 6

1.6. Bucky ball is a novel vertebrate specific germ plasm organizer protein................................... 8

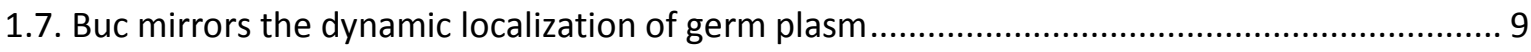

1.8. Buc induces ectopic germ cells after injection of mRNA into a somatic cell ............................. 11

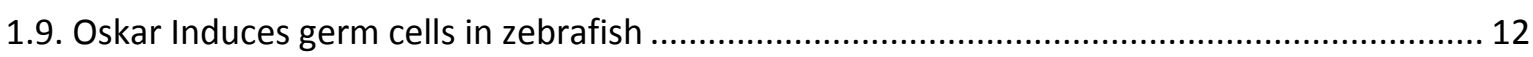

1.10. Buc and Oskar share conserved biochemical interaction during germ cell specification....... 14

1.11. The RNA helicase Vasa is a key player in germ cell specification........................................... 14

1.12. Structural characteristics of Dead box helicases.................................................................... 14

1.13. Buc interacts with zebrafish Vasa during germ cell specification ........................................... 16

1.14. Drosophila Oskar interacts with zebrafish Vasa in vitro ...................................................... 17

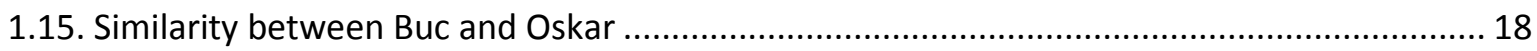

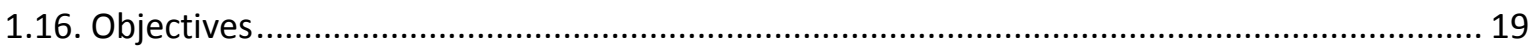

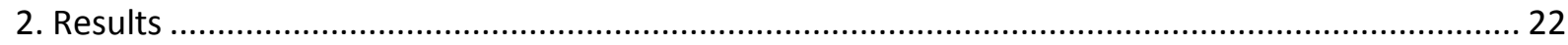

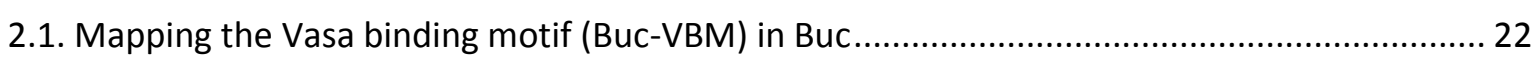

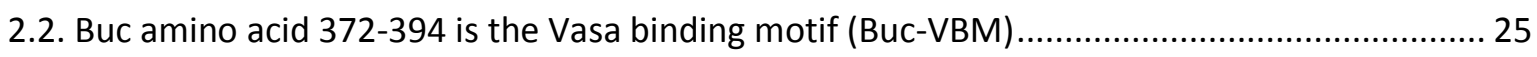

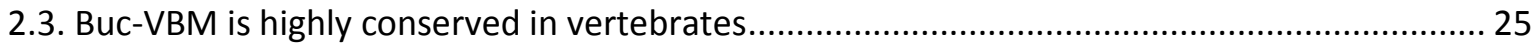

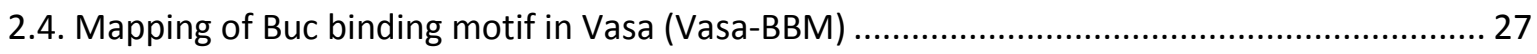

2.5. Buc-VBM and Vasa-BBM are required for their interaction .................................................. 31

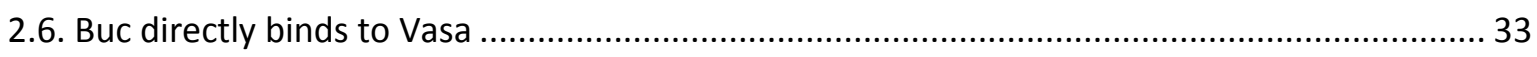

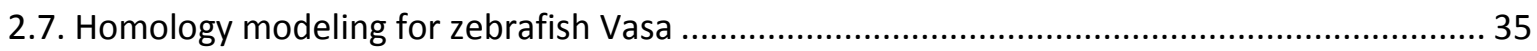

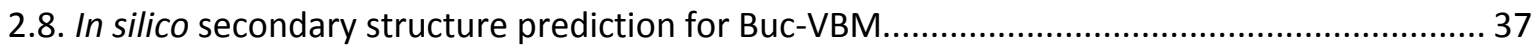

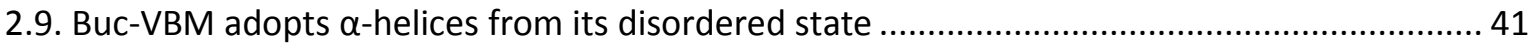

2.10. Buc-VBM is a novel activator of zebrafish Vasa helicase activity........................................... 43

2.11. Buc-Vasa binding motifs and intrinsically disordered region is not sufficient for germ cell

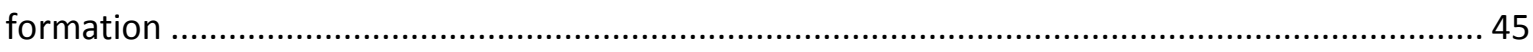

2.12. Buc-VBM and Vasa-BBM act as dominant negative proteins during germ cell specification. 47 
2.13. RNA activates zebrafish Vasa helicase activity.

2.14. Amino acid D379 in Buc is required for interaction with Vasa

2.15. Homology model predicts three residues in the Vasa-BBM potentially forming an interface during interaction with Buc 51

2.16. Vasa mutant for ATPase activity induces ectopic germ cells ...................................... 54

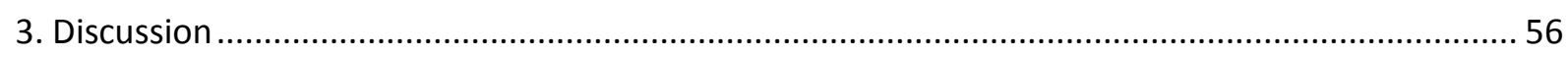

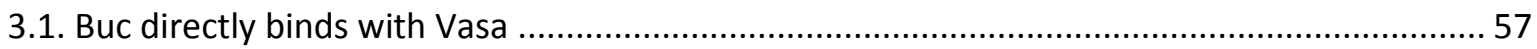

3.2. The novel interaction motifs in Buc and Vasa.............................................................. 58

3.3. Buc and Oskar share structural similarities............................................................ 59

3.4. Helicase activity of DEAD box protein is modulated by protein cofactors ............................62 62

3.5. The Oskar LOTUS domain and Buc-VBM stimulate the helicase activity of Vasa ....................62 62

3.6. Post-translational modification of Vasa and Buc is important for germ line development ..... 63

3.7. Importance of C-terminal region of Buc during germ cell specification ...............................64

3.8. Is the helicase activity of Vasa necessary for germ cell formation? ...................................67 67

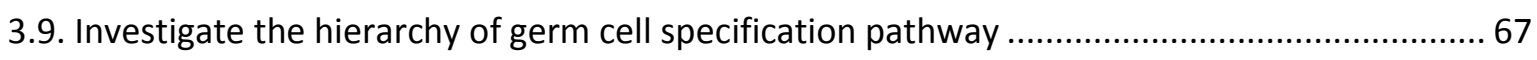

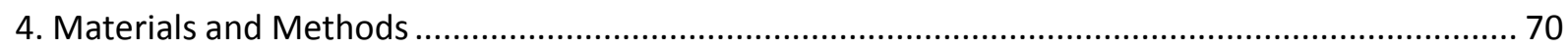

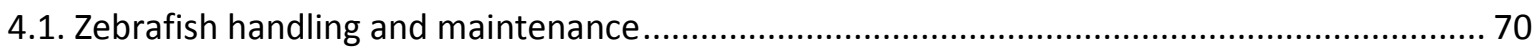

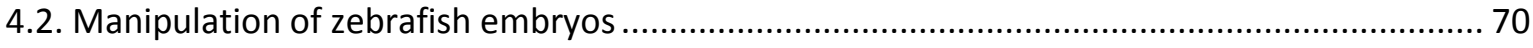

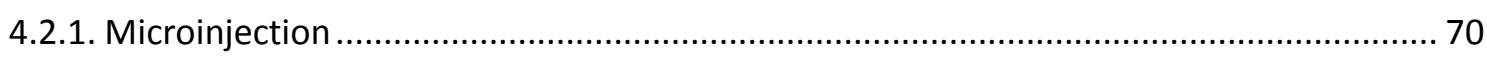

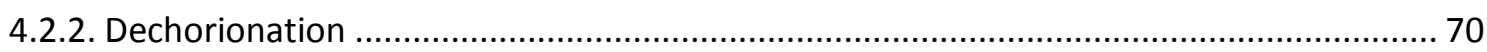

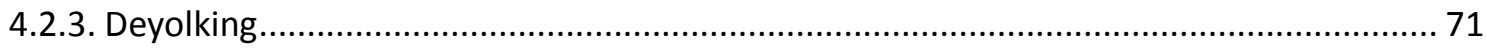

4.2.4. Preparation of embryo lysates.................................................................... 71

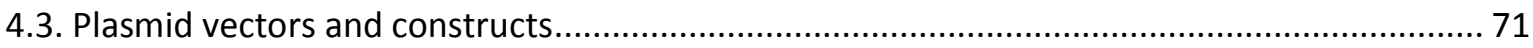

4.3.1. Plasmid vectors for In-Fusion cloning ............................................................... 71

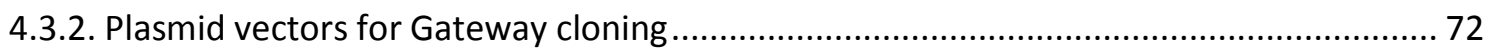

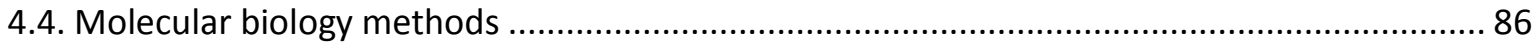

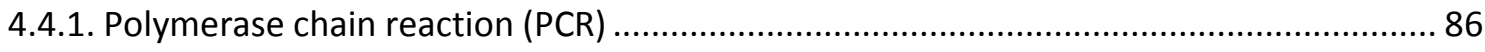

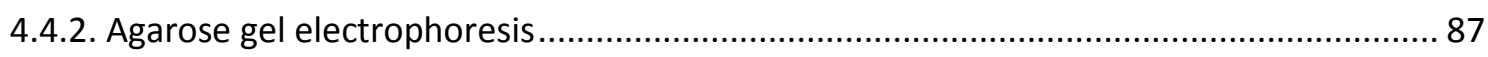

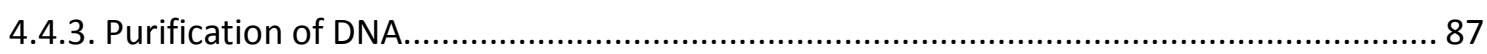

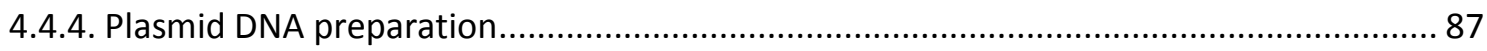

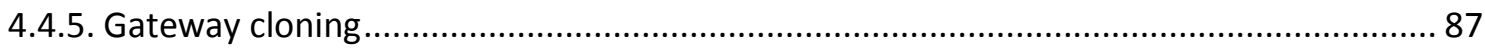

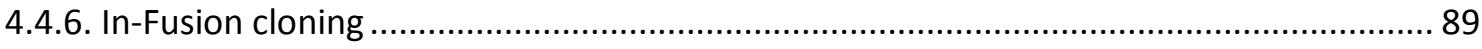

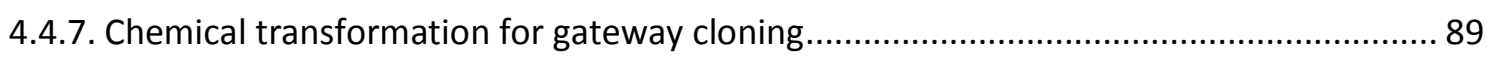

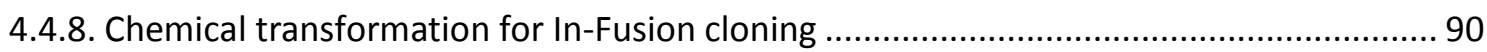

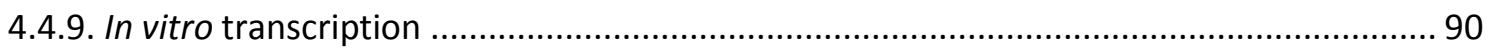

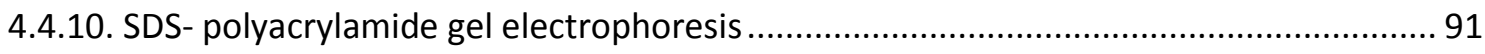

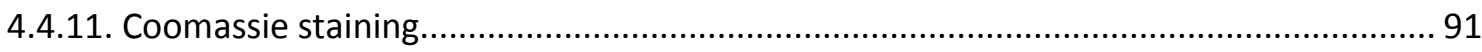

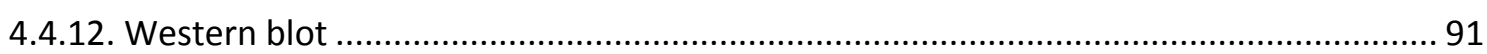

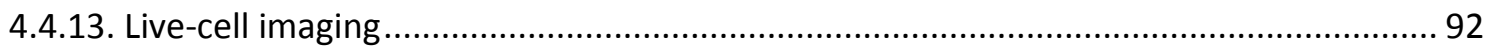




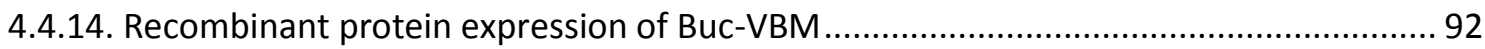

4.4.15. Recombinant protein expression of Vasa $(227-670)$ aa................................................... 93

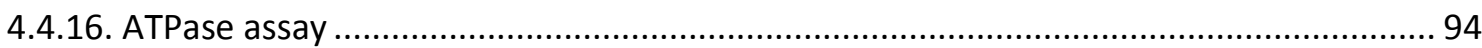

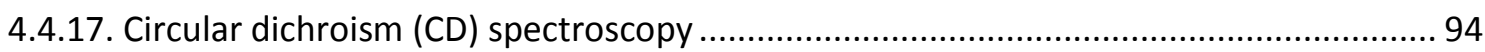

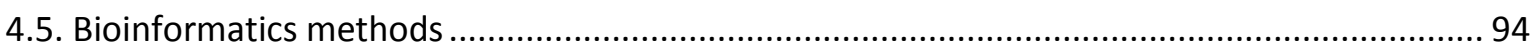

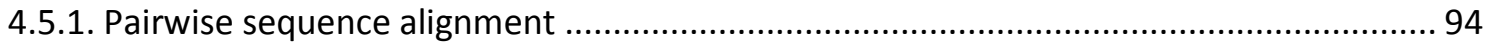

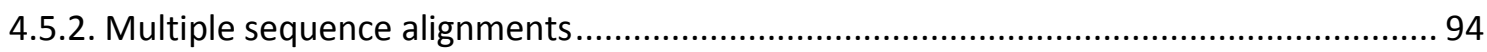

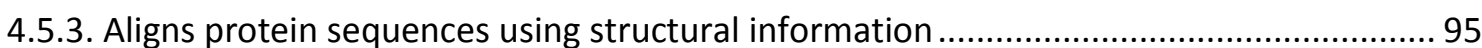

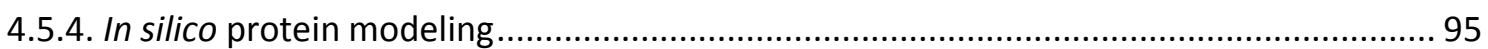

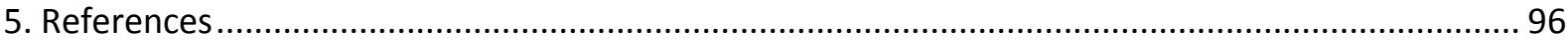

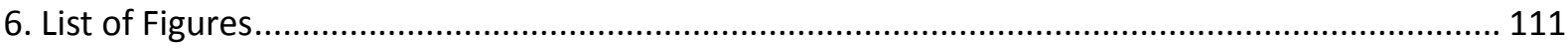

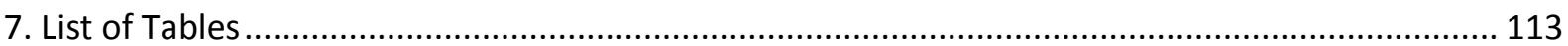

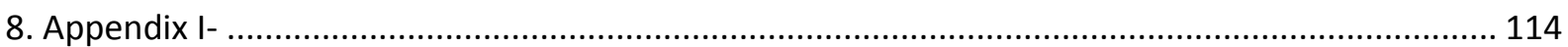

8.1. Pairwise sequence Alignment of Drosophila Vasa and zebrafish Vasa ................................... 114

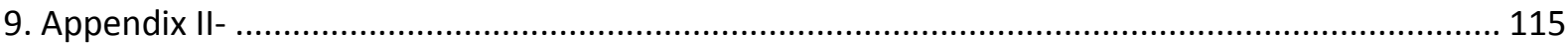

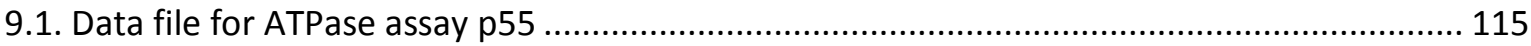

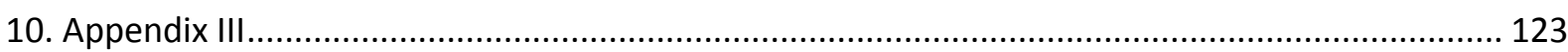

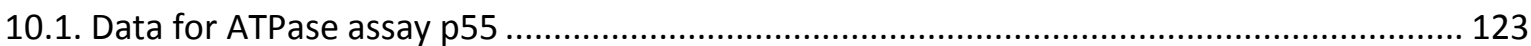




\section{Introduction}

Reproduction is an essential feature of life that ensures the perpetuation and survival in many multicellular organisms while passing the genetic information from one generation to the next (Santos \& Lehmann, 2004; Marlow, 2010; Jostes \& Schorle, 2018; Cieri et al., 2018).

\subsection{Sexual reproduction}

Sexual reproduction needs two parents to produce female and male germ cells i.e. eggs and sperm. During fertilization, egg and sperm fuse and form a zygote. The zygote undergoes embryonic development to form an offspring of the same species, but is genetically a combination of the parents (Dudgeon et al., 2017).

All germ cells arise from primordial germ cells (PGCs) (Dudgeon et al., 2017). In many animals, a population of PGCs segregates from the somatic cells during early embryonic development (Dudgeon et al., 2017). However, the mechanism of PGC specification is diverse among animal species (Santos \& Lehmann, 2004; Marlow, 2015). Nevertheless, two distinct mechanisms specify PGCs.

\subsection{Modes of germ cell specification}

The first mechanism is the induction mode of germ cell specification (Figure. 1). There, a subset of pluripotent embryonic cells is specified to PGCs by cell-cell communication through zygotic signaling molecules (Santos \& Lehmann, 2004; Carr, R. M., Oranu, A., \& Khungar, 2016; Marlow, 2015; Gustafson \& Wessel, 2010; Saitou \& Yamaji, 2010). This mode of germ cell specification can be seen in mammals such as mice and humans and some insects like Gryllus bimaculatus (cricket) (Extavour \& Akam, 2003; Nakamura \& Extavour, 2016; Krishnakumar \& Dosch, 2018; Marlow, 2015) 
A

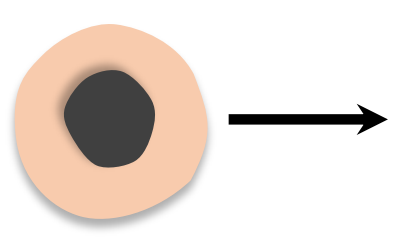

B

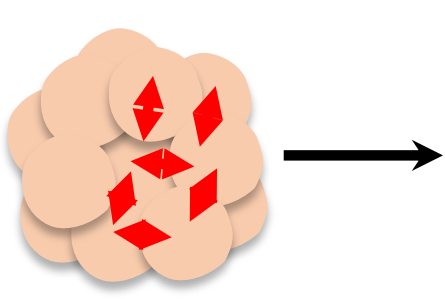

C

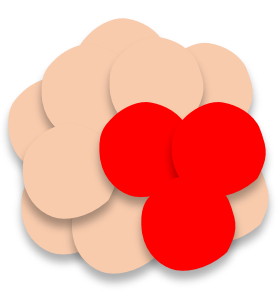

Figure 1 : Induction mode of germ cell specification.

A) Zygote (brown) and the nucleus (black). (B) Early embryogenesis with several blastomeres (light brown) and a subset of blastomeres sends inductive signals to each other to specify germ cells (red arrows). (C) Later stage of early embryo with specified PGCs (red)

The second mechanism is called inherited mode of germ cell specification (Figure. 2). In this mode, the newly fertilized, totipotent embryo inherits a cytoplasmic determinant referred as germ plasm (Gp). Gp is already deposited in the oocyte during oogenesis. During early embryogenesis, certain cells of the embryo inherit Gp, which develop into PGCs (Santos \& Lehmann, 2004; Carr, R. M., Oranu, A., \& Khungar, 2016; Marlow, 2015; Gustafson \& Wessel, 2010; Saitou \& Yamaji, 2010). Invertebrate species like Drosophila, C. elegans and vertebrate species like Xenopus, zebrafish, and birds display inherited mode of germ cell specification (Extavour \& Akam, 2003; Krishnakumar \& Dosch, 2018; Marlow, 2015).

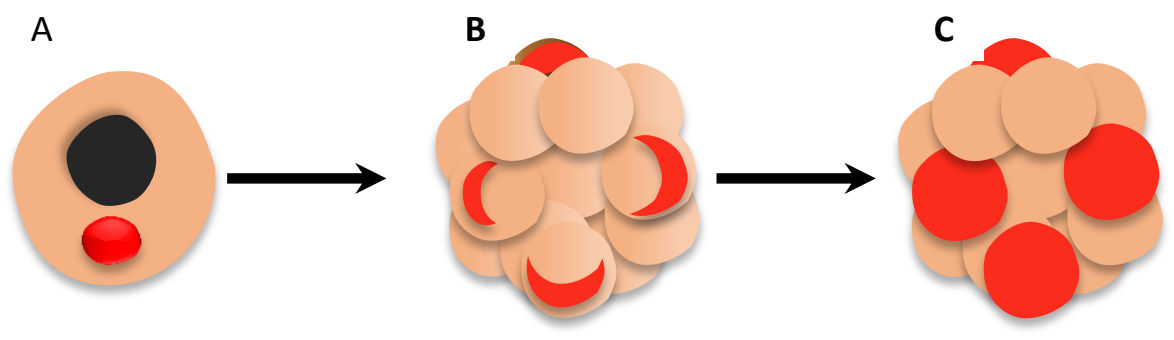

Figure 2: Inherited mode of germ cell specification.

(A) Oocyte (brown) with nucleus (black) and maternally inherited germ plasm (red). (B) Early embryogenesis with several blastomeres (brown). During the early embryogenesis, germ plasm (red) asymmetrically distribute among few blastomeres. (C) Later stage of early embryo with specified PGCs (red).

Regardless of the mode of specification, PGCs give rise to a continual supply of germ cells showing stemness properties like totipotency and immortality, that are distinct from those of somatic cells (Nakamura et al., 2019; Juliano et al., 2010). In addition PGCs express many 
conserved germ line genes such as Vasa, Nanos and Piwi among different species suggesting that the development of PGCs uses similar molecular mechanisms during evolution (Extavour \& Akam, 2003; Juliano et al., 2010; Liang et al., 1994). However, the molecular function of these conserved genes in PGC specification is poorly understood. In the past, Drosophila, C. elegans, and the mouse have been used as model organisms to understand the molecular mechanisms of germ cell specification. In this thesis, I used zebrafish as a model organism to investigate vertebrate germ cell specification as zebrafish combines the advantages of forward genetics as in Drosophila or $C$. elegans but is a vertebrate like the mouse.

\subsection{Germline development in zebrafish}

In zebrafish, PGCs are specified by the inheritance of maternal germ plasm (Figure. 3A). During oogenesis germ plasm is assembled in a huge ribonucleo-protein granule (RNP) also known as Balbiani body. The Balbiani body is assembled vegetal to the oocyte nucleus at the beginning of oogenesis (stage IB). Later during oogenesis in stage III oocytes, the Balbiani body is dispersed at the vegetal pole (Dosch, 2015; Kloc et al., 2014; Bontems et al., 2009; Heim et al., 2014; O.H. Kaufman and F.L. Marlow, 2017). The germ plasm comprises of maternally supplied RNAs, proteins, cell organelles like endoplasmic reticulum (ER), Golgi and mitochondria (Raz, 2003; Kloc et al., 2004; O.H. Kaufman and F.L. Marlow, 2017; Kloc et al., 2014; Marlow, 2015). After fertilization, vegetally localized germ plasm moves to the animal blastodisc during cytoplasmic streaming (Dosch, 2015).

Subsequent germ plasm positioning during early embryonic development has been demonstrated tracing molecular germ plasm markers such as vasa, nanos3, and dazl mRNA or Buc protein (Raz, 2000; Yoon et al., 1997; Braat et al., 1999; Hashimoto et al., 2004; Köprunner et al., 2001; Riemer et al., 2015). These molecular markers revealed that germ plasm localizes forming four spots at the distal end of the cleavage furrows after the first two cell cleavages (Figure. 3D). 


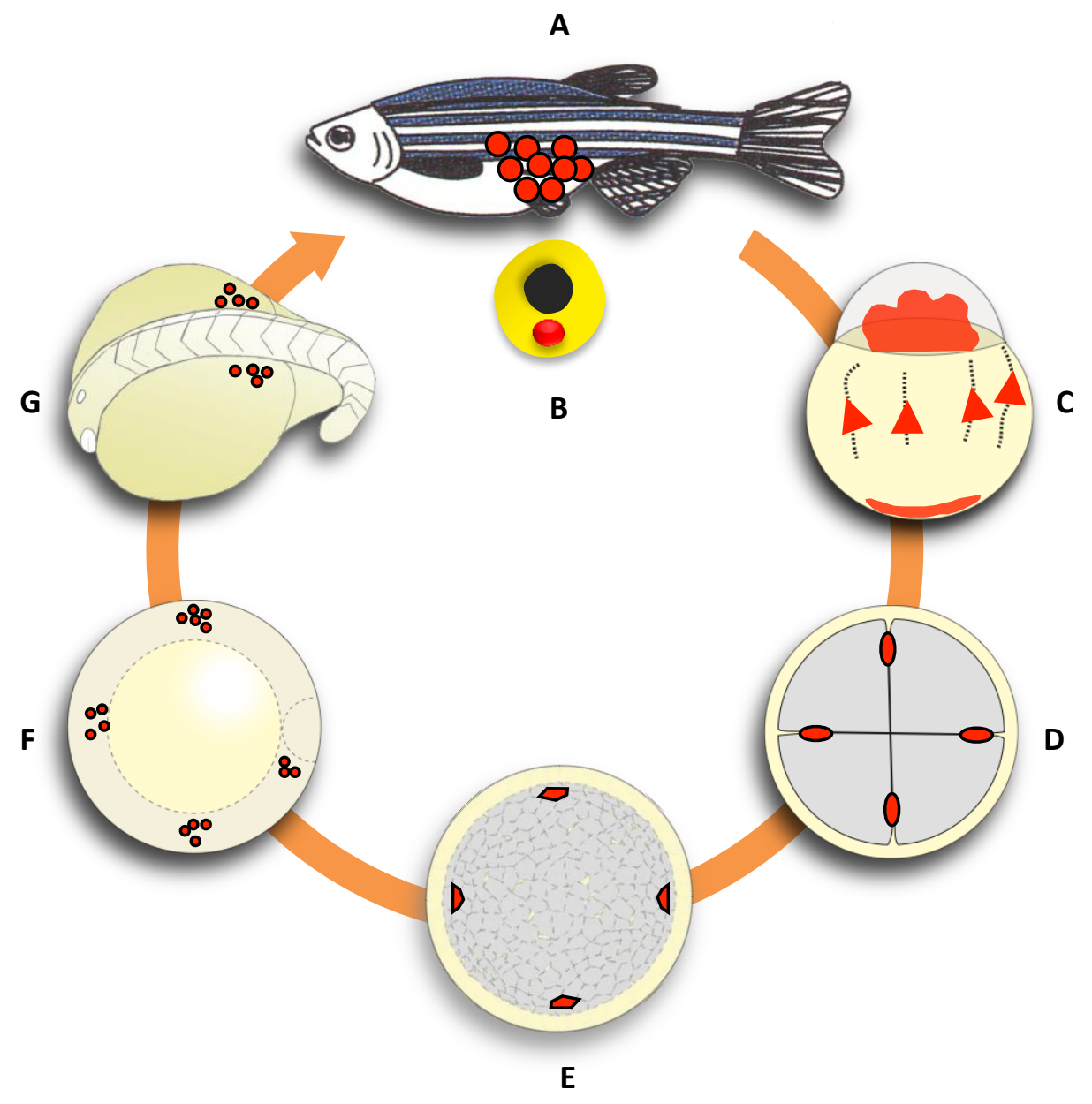

Figure 3: Germ line development of zebrafish.

(A) A female zebrafish with symbolized oocytes (red circles). (B) Stage I oocyte (yellow) with germ plasm/Balbiani body (red) vegetal to the nucleus (black). (C) Lateral view of fertilized egg, animal to the top. Red arrows indicate the migration of vegetal germ plasm to the animal blastodisc during cytoplasmic streaming. (D) Four-cell (grey) stage embryo, animal view. During the first two cleavages, germ plasm localizes to the distal region of the four cleavage furrows (red spots). However, no further germ plasm spots are formed during later stages. (E) $1 \mathrm{k}$-cell embryo ( $3 \mathrm{hpf}$ ), animal view. This is approximately after the $10^{\text {th }}$ cell division with around 1000 cells. Only four out of 1000 cells inherited germ plasm during asymmetric cleavage. These blastomeres are specified as PGCs (red). (F) Embryo in the gastrula stage with four PGCs clusters. These PGCs then start to migrate during further embryonic development. The dotted line on the right side denotes the shield, the zebrafish Spemann's organizer. (G) Dorsolateral view of embryo at 15-somite stage, head to the left and back to the top. Migrating PGCs form two clusters at either side of the midline, where the prospective gonads form. During sexual maturation, PGCs eventually differentiate and mature into sperm in males, or oocytes in females (A) to start another generation 
During subsequent cell divisions, germ plasm is asymmetrically inherited to one of the two daughter cells forming four cells with germ plasm at the 1'000-cell stage (3 hpf) (Figure. 3E). The other cells differentiate into somatic cells (Raz, 2003; Hashimoto et al., 2004; Riemer et al., 2015). After this embryonic stage of PGCs specification, germ plasm is symmetrically inherited between daughter cells during cell division forming four PGCs clusters (Figure. 3F). These PGCs then migrate to the prospective gonads (Figure. 3G) where they differentiate into mature male and female germ cells (Raz, 2000; Hashimoto et al., 2004; Dosch, 2015). Taken together, the inheritance of germ plasm into a subset of blastomeres during early embryogenesis is critical for the formation of PGCs, but also for the correct development of somatic tissues like neurons or the cardiovascular system.

\subsection{Germ plasm is critical for the formation of PGCs}

Intriguingly, many studies have shown that germ plasm plays a pivotal role for the formation of PGCs. For example, Ultraviolet (UV) irradiation of the vegetal pole of early anuran amphibian embryos Rana and Xenopus, led to complete absence of PGCs in the descendent tadpoles (Ikenishi et al., 1974; Smith, 1966; Tanabe \& Kotani, 1974). Further, surgically removal of vegetal germ plasm from Xenopus fertilized eggs caused to form sterile tadpoles (Buehr \& Blackler, 1970) demonstrating that germ plasm is essential for PGC development.

By contrast, microinjection of aspirated and purified germ plasm into UV-irradiated fertilized Rana eggs (Wakahara, 1977) and transplantation of Xenopus blastomeres containing microinjected germ plasm form PGCs in tadpoles (Ikenishi et al., 1986) demonstrating that germ plasm is sufficient to induce PGC development. Also, transplantation of EGFP labeled Xenopus germ plasm into animal blastomeres induced viable ectopic PGCs (Tada et al., 2012).

Similarly, after transplantation of Drosophila posteriorly localized pole plasm into the anterior end, recipient embryos form ectopic PGCs which are morphological and functionally similar to that of endogenous PGCs (IIImensee \& Mahowald, 1974). In addition, removal of 
cytoplasm containing germ plasm components at the ends of the cleavage furrows of fourcell stage zebrafish embryos resulted in a severe reduction in the number of germ cells (Hashimoto et al., 2004). Collectively, these results suggest that maternally deposited germ plasm factors are indispensable for the formation of PGCs in different animal species. These data also initiated several studies to identify the molecular nature of germ plasm components, which are necessary for the formation of PGCs.

\subsection{Roles of known germ plasm components during germ cell specification}

Germ plasm components required for germ plasm assembly and PGCs formation are well studied in Drosophila. For instance, Oskar, which is originally discovered from a genetic screen for maternal-effect posterior group genes in Drosophila is recognized as a gene necessary to assemble the germ plasm and germ cells at the posterior pole of the oocyte (Figure. 4B) (Lehmann \& Nüsslein-Volhard, 1986; Ephrussi, Anne; Lehmann, 1992). Further, it has been discovered that the overexpression of oskar mRNA to the anterior pole of the oocyte was sufficient to form ectopic germ plasm and functional germ cells at the anterior pole of the oocyte (Figure. 4C) (Smith et al., 1992; Ephrussi et al., 1991).

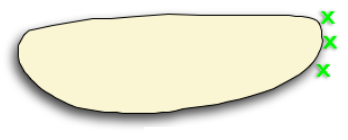

A

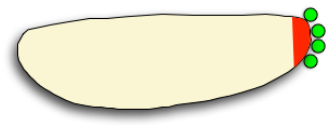

B

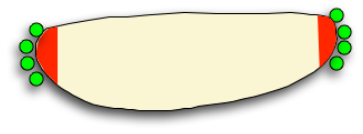

C

Figure 4: Drosophila Oskar is sufficient and necessary for germ cell formation.

All embryos are at stage 5 and shown in lateral view, anterior to the left. (A) Oskar mutant embryos do not assemble germ plasm and therefore do not form PGCs (green x). (B) In wild-type, Oskar assembles germ plasm at the posterior pole (red) and induces PGCs. (C) Ectopic expression of Oskar at the anterior pole forms extra germ plasm and thus induces ectopic PGCs.

In addition, the other germ plasm components such as Vasa, Staufen, Tudor and Valois have also been identified as essential genes for the assembly of germ plasm and germ cell formation in Drosophila (Schüpbach \& Wieschaus, 1986). Drosophila Vasa mutants fail to assemble germ plasm and hence, no PGCs form (Styhler et al., 1998; Lasko \& Ashburner, 
1988; Tomancak et al., 1998; Schupbach \& Wieschaus, 1986). Further, Vasa has been recognized as a translational regulator for gurken mRNA in oocytes and Mei-P26 mRNA in the ovary (Johnstone \& Lasko, 2004; Tomancak et al., 1998; Liu et al., 2009).

Moreover, Drosophila Vasa interacts with Piwi-interacting RNAs, piRNAs, to maintain the genome integrity of germ cells by silencing transposons (Pek et al., 2012; Xiol et al., 2014). Drosophila females mutant for tudor also abolish germ cell formation due to the failure of germ plasm assembly (Boswell \& Mahowald, 1985). The Drosophila RNA binding protein Staufen, which was identified in a genetic screen for the posterior group genes, is essential for the localization of osk mRNA to the posterior pole of the oocyte (Schüpbach \& Wieschaus, 1986; Ephrussi et al., 1991; Kim-Ha et al., 1991; St Johnston et al., 1991). These data conclude that the germ plasm components are essential for the assembly of germ plasm and also ochestrate germ cell formation.

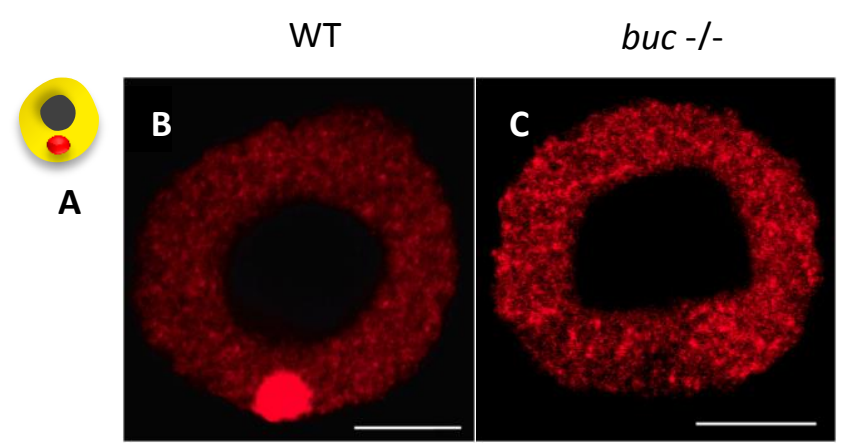

Figure 5: Buc is required to organize germ plasm in zebrafish.

(A) A cartoon of a lateral view of a stage I oocyte (yellow) showing germ plasm (Balbiani body; red) vegetal to the nucleus (dark grey). (B) Lateral view of stage I wild-type oocyte. Fluorescent in-situ hybridization against germ plasm marker, nanos3 mRNA highlights aggregated germ plasm (red) vegetal to the nucleus (black circle). (C) Buc mutants are unable to organize germ plasm but instead nanos3 mRNA is dispersed ubiquitously in the ooplasm (red patches). Scale bar is $500 \mu \mathrm{m}$. This image modified from (Bontems et al., 2009). 


\subsection{Bucky ball is a novel vertebrate specific germ plasm organizer protein}

The germ plasm organizer bucky (buc) ball gene was discovered in a forward genetic maternal-effect screen in zebrafish (Dosch et al., 2004; Wagner et al., 2004). Oocytes produced by buc mutant females do not have a properly aggregated Balbiani body; instead, the RNP granules are ubiquitously dispersed in the ooplasm (Figure 5). This interprets buc as the first gene which is necessary for Balbiani body formation in vertebrates (Dosch et al., 2004; Bontems et al., 2009). Moreover, buc mutant oocytes and eggs exhibited a defect in animal-vegetal polarity (Dosch et al., 2004; Florence L. Marlow and Mary C. Mullins, 2008) (Figure 6). Therefore, embryos with polarity defects do not develop beyond the one-cell stage (Dosch et al., 2004).
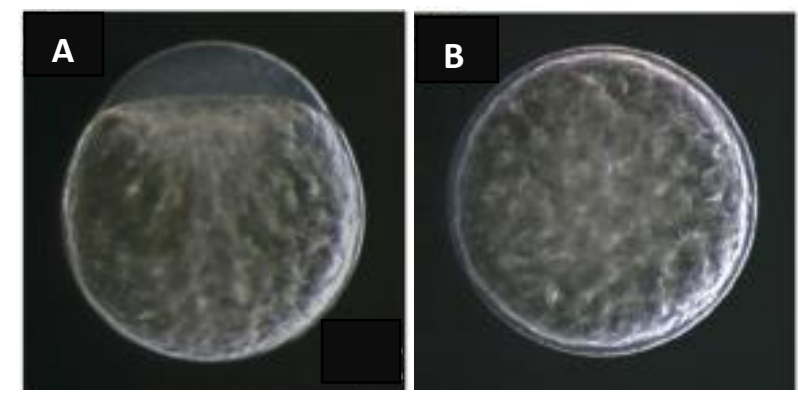

Figure 6: Phenotype of buc wild-type and buc mutant embryos.

buc mutants show a defect in embryonic animal-vegetal polarity. (A) In the wild-type embryo, the blastodisc is formed at the animal pole due to cytoplasmic streaming. (B) The buc mutant embryo does not form blastodisc at the animal pole, instead cytoplasm radially accumulates around the central yolk. Embryos at 30 (minutes post fertilization) mpf are shown with animal to the top. This image modified from Dosch et al. (2004).

Mapping the isolated buc mutation to the genome and positional cloning of the Buc cDNA revealed a novel gene in zebrafish (Bontems et al., 2009). Examining the conservation of the buc gene by BLAST searches and synteny identified homologous sequences for Buc in all vertebrate classes such as fish, amphibian, birds and mammals (Bontems et al., 2009). Further, alignment of 15 Buc related proteins from different vertebrates discovered two highly conserved N-terminal sequence motifs within the conserved BUVE motif (Krishnakumar et al., 2018) (Figure. 7). 
In addition to BUVE motif, the same sequence alignment identified another conserved motif, which shows higher sequence conservation corresponding to amino acid 372-394 (Figure 7). However, the function of this highly conserved motif in Buc is still unknown. Therefore, Buc represents a novel vertebrate specific protein with powerful biological activities whose biochemical function still needs to be uncovered (Bontems et al., 2009).

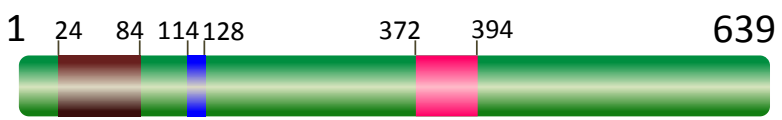

Figure 7: Schematic representation of evolutionary conserved motifs in Buc.

Buc protein (green bar) contains three conserved motifs. The N-terminal BUVE motif (amino acid 23 - 136) comprises two sub motifs encoding amino acid 24-84 (brown box) and 114-128 (blue box) and the highly conserved motif encoding amino acid 372-394 (pink) adopted from (Krishnakumar et al., 2018)

\subsection{Buc mirrors the dynamic localization of germ plasm}

Using a novel anti-Buc antibody, we labeled the germ plasm of zebrafish (Riemer et al., 2015). In wild-type oocytes, anti-Buc antibody specifically recognized the Balbiani body (Figure $8 \mathrm{~A}$ ) but not the mutant buc $P^{106}$ (Figure 8B).

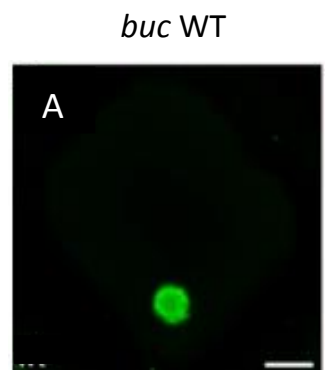

buc $P^{106}$

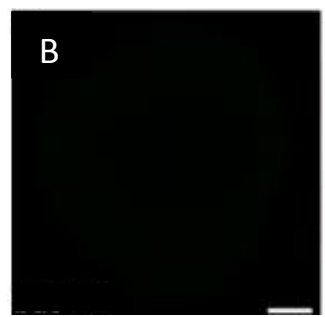

vasa

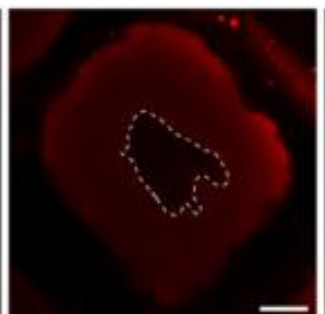

vasa

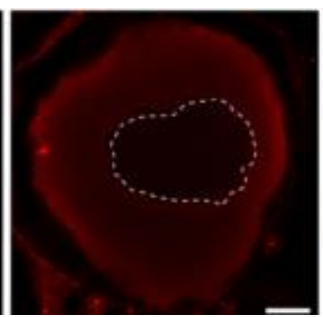

merge

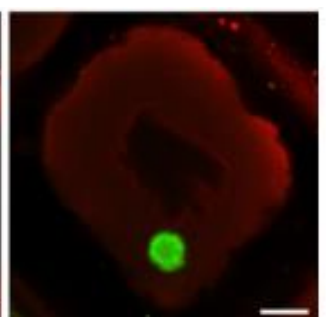

merge

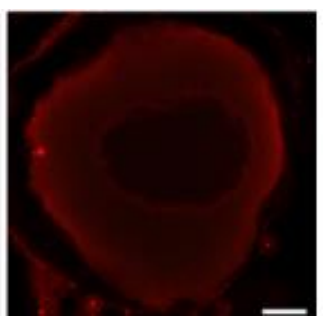

Figure 8: Anti-Buc antibody specifically recognize Buc.

Upper and lower panel shows the lateral view of stage IB oocytes, animal to the top. (A) Anti Buc antibody recognizes the Buc protein in the Balbiani body (green) but not mutant BucP ${ }^{106}$ (B). (C) Besides, perinuclear Vasa is not changed. Nuclear is demarcated in dashed line. Scale bar $10 \mu \mathrm{m}$. (adopted from (Riemer et al., 2015). 
Further, dynamic of germ plasm localization have been investigated labeling germ plasm using anti-Buc antibody (Figure 9) (Riemer et al., 2015).
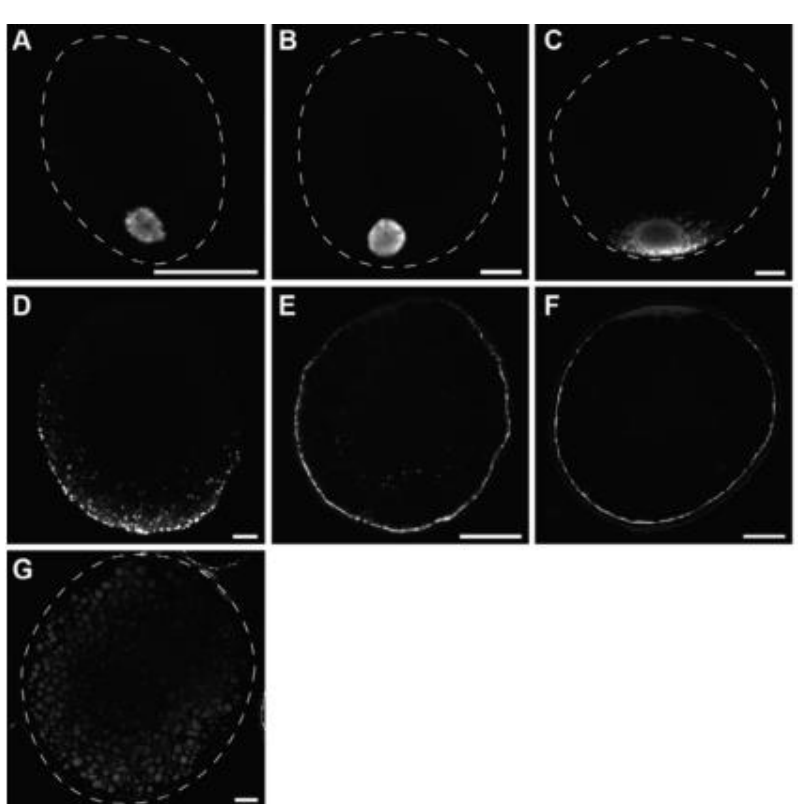

Figure 9: Dynamic of Buc localization during oogenesis

Images represent the lateral view of stage IA (A), early IB (B), late IB (C, D), early II (E), late II (F) and III (G), animal to the top. Buc protein is labeled with anti-Buc antibody (white). During oogenesis, Buc localizes with the germ plasm to the vegetal pole. Stippled line is denoted outline of oocytes. Scale bar $10 \mu \mathrm{m}$. This image modified from (Riemer et al., 2015)

In addition, overexpression of buc-GFP mRNA into early oocyte revealed that Buc localizes to the Balbiani body and later in oogenesis, Buc actively localize more to the vegetal pole of the oocyte. Similarly, overexpression of buc-GFP mRNA into once-cell stage embryos divulged that Buc-GFP aggregate in the four cleavage furrows at the eight-cell stage (Bontems et al., 2009). Fascinatingly, Buc-GFP transgenic fish are reminiscent of the overexpression results. (Figure 10) (Riemer et al., 2015). Therefore, Buc mirrors the dynamic of germ plasm localization during oogenesis and early embryogenesis, which is consistent with its function as a germ plasm organizer. 

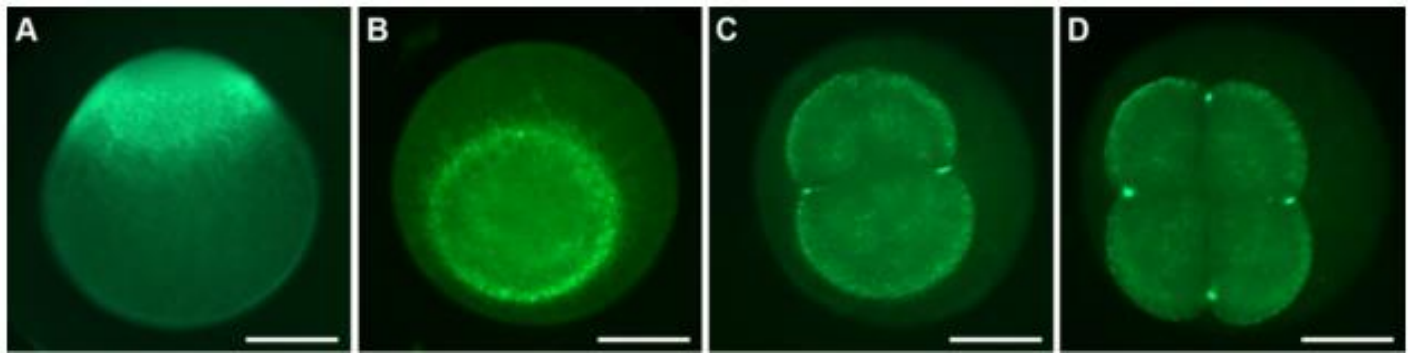

Figure 10: Buc-GFP transgenic embryos mimics the dynamics of germ plasm localization.

One-cell stage embryos show fluorescent blastodics (A: lateral view, B: animal view). At the two-cell stage (B: animal view) and four-cell stage (D: animal view) germ plasm localize to the distal cleavage furrow. Scale bar $200 \mu \mathrm{m}$. ( adopted from (Riemer et al., 2015).

\subsection{Buc induces ectopic germ cells after injection of mRNA into a somatic cell}

As mentioned above, buc-GFP overexpression leads to its aggregation at four spots at the eight-cell stage. Beyond the eight-cells stage, the spots remain as four foci until high stage suggesting that Buc specifies germ cells in vertebrates (Bontems et al., 2009). Indeed, the germ cell induction assay (16-cell assay) revealed that Buc expression in a somatic cell induces ectopic formation of germ cells during early embryogenesis (Bontems et al., 2009) (Figure 11). Such an in vivo activity was hitherto not described for any vertebrate gene. 
A

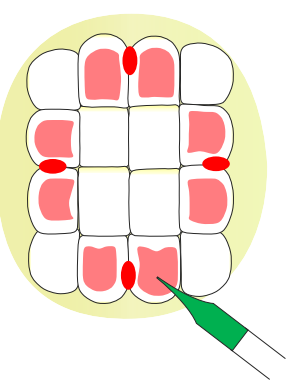

$A^{\prime}$

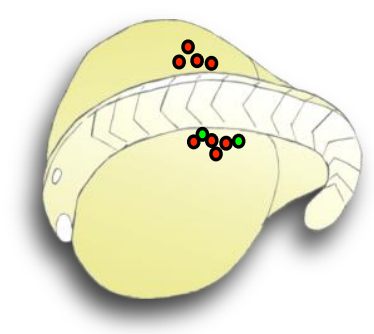

B

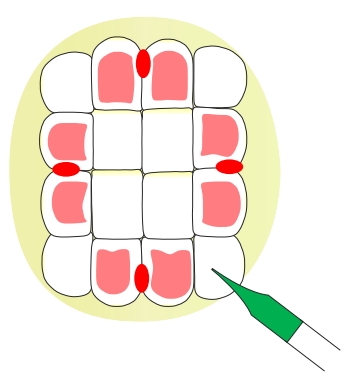

$B^{\prime}$

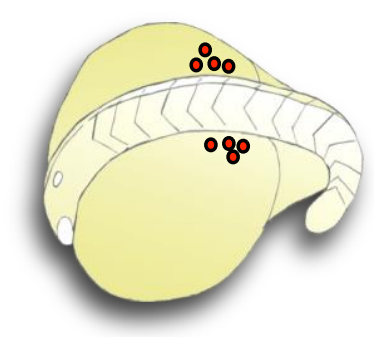

C

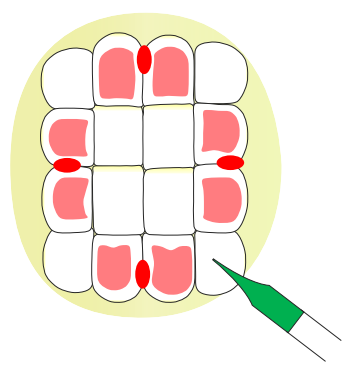

$\mathbf{C}^{\prime}$

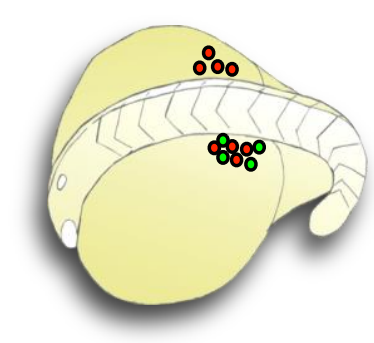

Figure 11: Schematic illustration of germ cell induction assay.

Upper panel (A-B) shows embryos at the 16-cell stage (animal view) with localized germ plasm (red oval) together with potential to form future PGCs (middle blastomeres in light red). After injection (green needle tip), embryos were examined for germ cells after 15-18 somite stage. Lower panel $\left(A^{\prime}-C^{\prime}\right)$ illustrates the activity of the germ cell reporter (green) in an 18-somite stage embryo (dorsolateral view, anterior to the left). (A) Injection of PGC reporter (GFPnanos3-3'UTR mRNA; citation) into a middle cell as a positive control and ( $\left.A^{\prime}\right)$ embryos show a fraction of endogenous germ cells positive for PGC reporter (green circles) next to unlabeled germ cells (red circle; invisible in the experiment). (B) Injection of reporter RNA into a somatic, corner blastomere as a negative control leads to embryos ( $\mathrm{B}^{\prime}$ ) without fluorescent PGCs but endogenous germ cells (red circles). (C) Injection of reporter RNA together with buc mRNA into a somatic, corner blastomere leads to embryos $\left(C^{\prime}\right)$ whose somatic cells are reprogrammed into extra PGCs (green circles) in addition to the endogenous germ cells (red).

\subsection{Oskar Induces germ cells in zebrafish}

After localization to the posterior pole of the oocyte, Drosophila oskar mRNA is translated into two isoforms, Long (IOskar) and Short Oskar (sOskar) (Vanzo \& Ephrussi, 2002). However, only sOskar is responsible for the assembly of germ plasm and formation of germ cells whereas IOskar anchors oskar mRNA and sOskar protein the posterior pole (Vanzo \& Ephrussi, 2002).

As stated, Buc is a vertebrate specific novel protein without having any sequence homology for other proteins with known functions (Riemer et al., 2015). Thus, understanding the 
molecular function of Buc in germ cell specification is a great challenge. Thus, finding a protein in which the genetic function is evolutionary conserved provide an alternative approach to investigate function of a novel protein (Jensen et al., 2003). Therefore, our lab used an innovative approach in which Oskar was used as a functional homolog without related amino acid sequence to investigate the function of Buc in germ cell specification (Bontems et al. 2009).

Fascinatingly, overexpression of Drosophila sOskar mRNA (amino acid 139-606) induces ectopic germ cells in the zebrafish germ cell induction assay (Figure 12C), whereas mutant sOskar (osk ${ }^{84}$, amino acid 139-254) was unable to induce ectopic germ cells (Krishnakumar et al., 2018). This result is reminiscent of the overexpression of wild-type (amino acid 1-639) and mutant buc (buc ${ }^{p 43}$ amino acid 1-362) mRNA. In this assay, wild-type buc mRNA induced ectopic germ cells but not mutant buc (Bontems et al., 2009; Krishnakumar et al., 2018). Both osk ${ }^{84}$ and buc ${ }^{p 43}$ mRNA have the identical nucleotide sequence to wild type with only one nucleotide change generating a premature STOP codon (Kim-Ha et al., 1991; Bontems et al., 2009). This reveals that Buc and sOskar but not their RNA is sufficient for germ cell induction.

Surprisingly, Oskar and Buc do not share sequence homology although they perform the same biological activity (Krishnakumar et al., 2018). Therefore, these results reveal for the first time the functional equivalence of two germ plasm organizers in germ cell specification without sequence homology (Krishnakumar et al., 2018). More importantly, these results suggest that sOskar and Buc use a similar biochemical mechanism to induce germ cells in zebrafish.

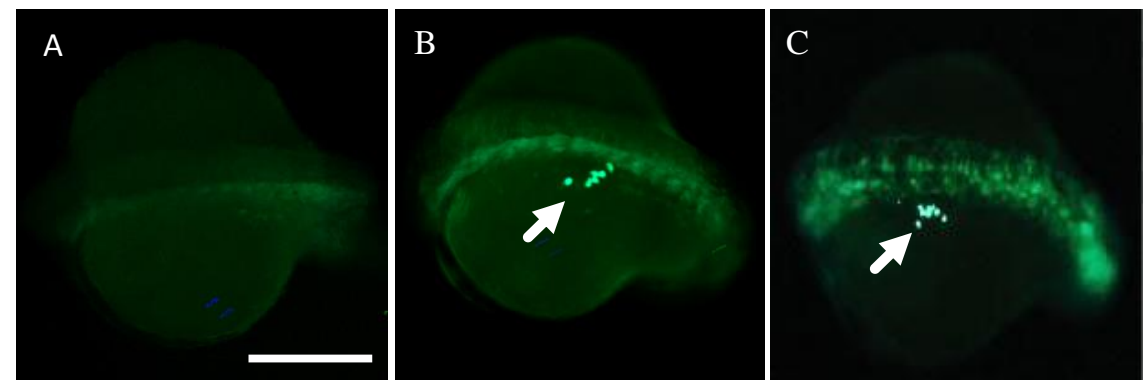

Figure 12: Buc and Oskar in germ cell induction assay.

Dorsolateral view of living embryos at 15-18 somite stage, anterior to the left. (A) Injection of PGC reporter (GFP-nano3-3' UTR) into a corner blastomere does not induce extra germ cells. Co-injection of reporter together buc (B) or soskar mRNA (C) induces extra fluorescent germ cells (green, white arrow). 


\subsection{Buc and Oskar share conserved biochemical interaction during germ cell specification}

As described previously, proteins with similar amino acid sequences share a similar biological function. Surprisingly, we showed functional equivalence between Drosophila sOskar and zebrafish Buc even though they do not share sequence homology. Nonetheless, this result postulates that both sOskar and Buc likely share similar biochemical interactions during the formation of germ cells. Oskar binds to Smaug, Valois and Vasa proteins (Dahanukar et al., 1999; Anne, 2010; Breitwieser et al., 1996). Especially the direct Vasa - Oskar binding has been a major focus, because this interaction is essential for germ plasm assembly and germ cell specification (Breitwieser et al., 1996; Jeske et al., 2015).

\subsection{The RNA helicase Vasa is a key player in germ cell specification}

Vasa was originally discovered from a genetic screen for maternal-effect genes in Drosophila required for the formation of germ cells (pole cells) and abdominal segments (Schupbach \& Wieschaus, 1986). Thereafter, Vasa has been identified as a conserved germ cell specific protein in variety of animal taxa such as sponges, cnidarians, flatworms, annelids, nematodes, echinoderms, tunicates, mollusks, insects, crustaceans, fish, amphibians, reptiles, birds, and mammals (Hickford et al., 2011). As a result of this conservation, Vasa is used nowadays as a universal marker for germ cell lineage (Gustafson \& Wessel, 2010; Hickford et al., 2011; Raz, 2003). Functionally, Vasa, aka DDX4 (DEAD [Asp-Glu-Ala-Asp] box polypeptide 4), is an ATP-dependent RNA helicase belonging to the DEAD box protein family (Linder, 2006; Hickford et al., 2011).

\subsection{Structural characteristics of Dead box helicases}

DEAD box family RNA helicases form the largest helicase family and are found in all three kingdoms of life i.e. Bacteria, Archea and, Eukaryotes (Jankowsky, 2011). Essentially, these helicases participate in nearly all aspects of RNA metabolism such as transcription, translation initiation, ribosome biogenesis, splicing, RNA editing, RNA export from the nucleus, and RNA degradation. In these processes, RNA helicases remodel higher-order RNA 
secondary or tertiary structures and RNA-protein complexes in an ATP dependent manner (Jankowsky, 2011; Linder, 2006; Linder \& Lasko, 2006; Popovic et al., 2019; Linder \& Jankowsky, 2011).

DEAD box RNA helicases share a structurally highly conserved helicase core, which comprises of almost two identical RecA like domains. Each RecA like domain resembles that of bacterial recombination protein RecA and is connected by a flexible linker, which supports conformational changes of the helicase core during their catalytic activity (Linder, 2006; Jankowsky, 2011; Linder \& Jankowsky, 2011). In addition, this protein family shows variable $\mathrm{C}$ - and N-terminal extensions on either sides of the helicase core which facilitates binding for additional protein-protein interactions (Linder \& Jankowsky, 2011; Sloan \& Bohnsack, 2018). The helicase core possesses at least 12 characteristic sequence motifs, which provide binding sites for ATP and RNA (Figure. 13) (Sloan \& Bohnsack, 2018; Linder \& Jankowsky, 2011; Linder \& Lasko, 2006). Motifs I, la, Ib, II, and III are located in the N-terminal RecA-like domain (NTD) while motifs IV, V, and VI are located in the C-terminal domain (CTD) (Caruthers \& McKay, 2002; Hickford et al., 2011). The motif II, which harbors the conserved four amino acids, Asp-Glu-Ala-Asp, led to the designation "DEAD box" for this protein family (Linder \& Jankowsky, 2011).

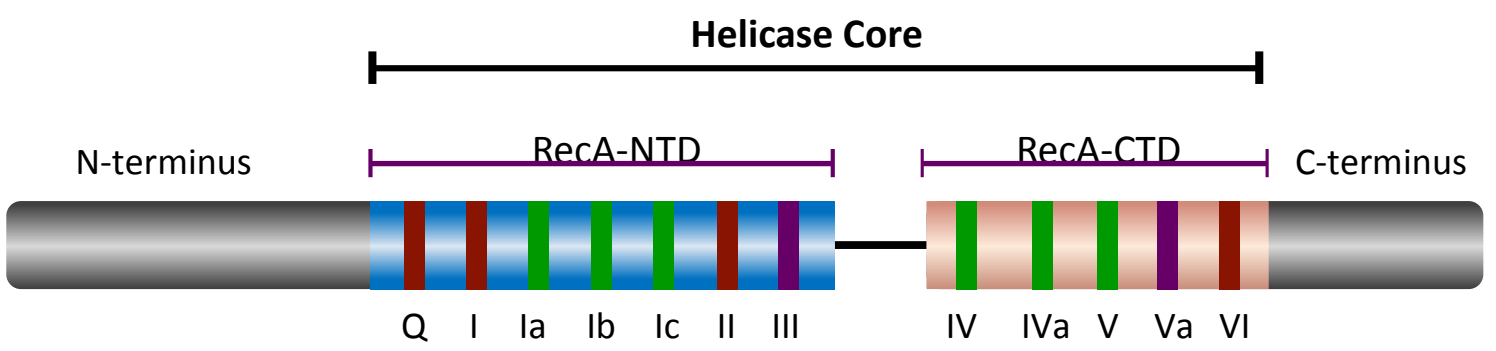

Figure 13: Schematic illustration of general features of DEAD box RNA helicase.

Dead box RNA helicases comprise a highly conserved helicase core. The Helicase core contains two RecA-like domains, RecA-NTD (Blue) and RecA-CTD (Salmon), which are connected with a flexible linker sequence (black line). Either side of the helicase core has $\mathrm{N}$ - and C-terminal flanking sequences (grey). Conserved sequence motifs located in each RecA-like domain are shown as horizontal colored boxes according to their primary functions, such as ATP binding and hydrolysis motifs (brown), RNA binding motifs (green) and motifs for communication between ATP binding and duplex unwinding (purple). This image modified from (Jankowsky, 2011). 


\subsection{Buc interacts with zebrafish Vasa during germ cell specification}

It has been shown that the Oskar-Vasa interaction is required to promote efficient oskar mRNA translation and phosphorylation of Oskar protein (Breitwieser et al., 1996). Further, it has also been reported that the LOTUS domain of Oskar directly binds the RecA like Cterminal domain of Drosophila Vasa. The binding of the LOTUS domain stimulates the ATPase activity of Vasa (Jeske et al., 2017, 2015)

Analysis of the Buc interactome using mass spectrometry discovered that zebrafish Vasa is highly enriched in the Buc-GFP pull-down assay (Krishnakumar et al., 2018). This enrichment suggests that Buc and Vasa might also interact with each other. Co-immunoprecipitiation indeed indicates that Buc and Vasa interact in vivo during germ cell specification confirming the mass spectrometry data (Figure. 14) (Krishnakumar et al., 2018). Further, it has been demonstrated that Buc and Vasa localization overlaps in the germ plasm during early embryogenesis including the period of germ cell specification (Krishnakumar et al., 2018). In chicken, it has been shown that overexpression of the Vasa homologue, Cvh reprograms embryonic stem cells to PGCs (Lavial et al., 2009). This data raised the hypothesis that Vasa has a critical role during germ cell specification in zebrafish. Indeed, overexpression of vasa mRNA in the zebrafish germ cell induction assay revealed that Vasa also induces ectopic germ cells identifying Vasa as the second protein with a germ cell specification activity (Krishnakumar et al., 2018). 


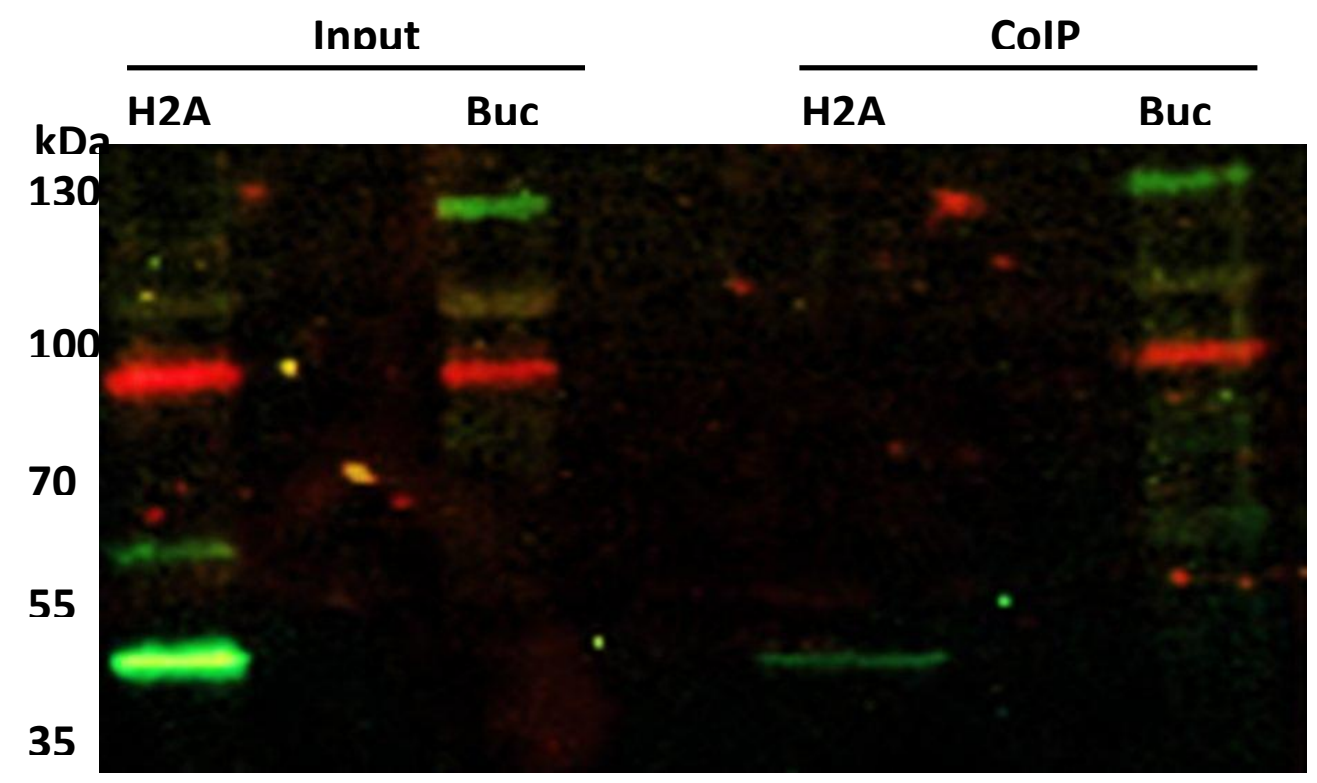

Figure 14: Buc interacts with Vasa during germ cell specification.

Western-blot after co-immunoprecipitation has been performed using Buc-GFP transgenic embryos. H2A-GFP transgenic line used as a control. Embryos at $3 \mathrm{hpf}$ have been collected from both fish line. GFP pulldown assay has been performed after lysing cells. Input samples show the expression of H2A-GFP (42 kD, green band), Buc (130 kD, green band) and Vasa (80 kD, red band) presence in the sample. However, co-immunoprecipitation samples show only Buc-GFP pulldown Vasa but not H2A-GFP.

\subsection{Drosophila Oskar interacts with zebrafish Vasa in vitro}

With aforementioned findings, it is reasonable to hypothesize that ectopic germ cell formation by sOskar is mediated through zebrafish Vasa. To explore that, the coding sequence of sOskar fused to GFP (sOskar-GFP) and zebrafish Vasa were transcribed and translated in a cell free system followed by GFP pulldown assay. Results showed that sOskar interacts with zebrafish Vasa in vitro (Figure 15) supporting to the aforementioned hypothesis (Krishnakumar et al., 2018). 


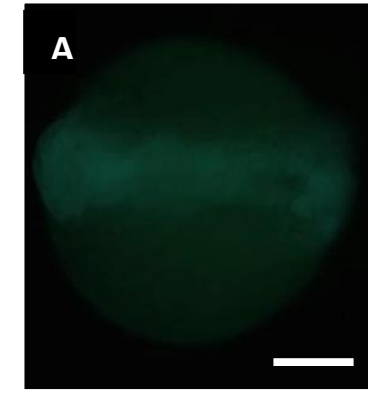

Hermes

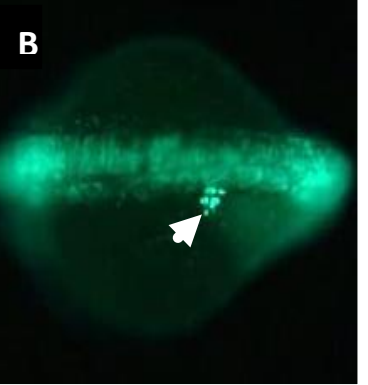

Vasa

Figure 15: Vasa induce ectopic germ cell in zebrafish.

Dorsolateral view of embryo at 15-18-somite stage, animal to the left (A) Injection of Buc interacting protein, Hermes (Heim et al., 2014) into the corner blastomere in 16 cell stage embryos does not induce germ cells. (B) Injection of vasa mRNA into the corner cell induces ectopic germ cells (white arrow). Scale bar is $100 \mu \mathrm{m}$.

\subsection{Similarity between Buc and Oskar}

Buc is recognized as a novel vertebrate specific protein while Oskar was exclusively found in invertebrates like Drosophila (Bontems et al., 2009). Although Buc and Oskar do not share sequence homology, it seems that they have common biochemical functions. For instance, both proteins organize germ plasm and play a crucial role in germ cell formation. Mutant alleles of both proteins end up with a failure in germ plasm assembly and polarity defects in oocytes and embryos. In addition, Buc and Oskar can induce ectopic germ cells. Moreover, both Buc and Oskar interact with Vasa in vivo and in vitro (Jeske et al., 2015; Krishnakumar et al., 2018; Jeske et al., 2017). In addition, Buc and Oskar are composed of low complexity sequences hence predicted as intrinsically disordered proteins (Figure 16). 
A

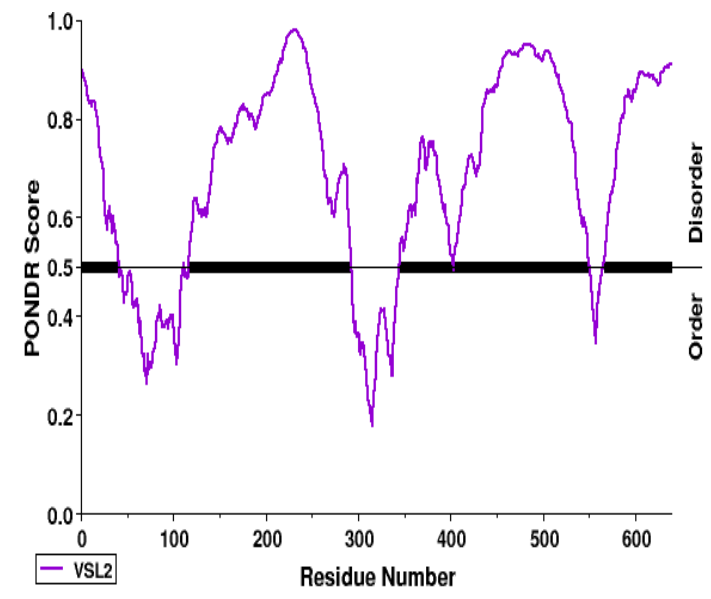

B

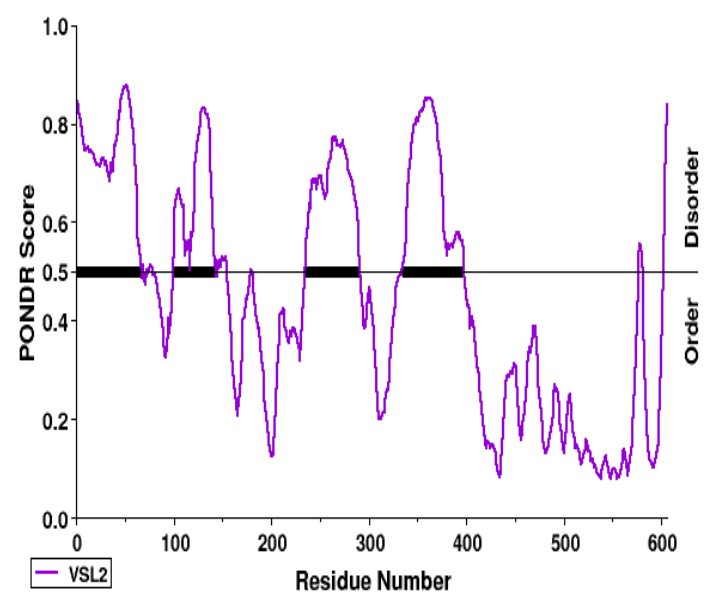

Figure 16: Buc and Oskar are intrinsically disordered proteins.

Intrinsically disordered properties of Buc (A) and Oskar (B) predicted using PONDR protein disorder predictor using VSL2 algorithm. Y-axis represents the generated disordered PONDR score. X-axis represents the number of amino acids for a given protein. Threshold value between ordered and disordered protein considered as 0.5 (thick horizontal black line). Distribution of the disordered and ordered properties are representing in purple colour. Protein regions above the threshold value are ordered while regions below the threshold value are disordered.

\subsection{Objectives}

Overexpression of Oskar in zebrafish induces ectopic germ cell formation. However, Buc and Oskar do not share sequence homology (Krishnakumar et al., 2018). This shows for the first time that two evolutionary distance proteins with no sequence homology perform equivalence biochemical function in germ cell specification (Krishnakumar et al., 2018). Therefore, we speculated Buc and Oskar have a conserved interactome in germ cell specification. To that end, it is revealed that Buc and Oskar interact with Vasa, a highly conserved germ plasm component across species, during germ cell specification. Interaction between Oskar and Vasa is exclusively characterized by different aspects. For example, it has been discovered that the LOTUS domain of Oskar physically binds to RecA like C-terminal domain of Drosophila Vasa (Jeske et al., 2017). Moreover, it has also been shown that Oskar LOTUS domain stimulates Vasa ATPase activity upon their interaction (Jeske et al., 2017). As Buc and Oskar are equivalent in function, I hypothesized that Buc and zebrafish Vasa are likely to perform similar biochemical function during germ cell specification. Thus far, there 
is no known protein motif in Buc which interacts with Vasa or vice versa. Furthermore, no known biochemical activity is annotated to Buc apart from Buc as a scaffolding protein bringing together RNA binding proteins such as Hermes (Heim et al., 2014). Therefore, the aim of this work is to characterize the Buc and Vasa interaction during germ cell specification with following objectives.

\section{Determine whether Buc and Vasa directly bind during germ cell specification}

In vitro co-immunoprecipitation data and in vivo BiFC data clearly show that Buc interacts with Vasa during germ cell specification. So far, there are no studies characterizing Buc and Vasa interaction during zebrafish germ cell specification. Therefore, there is no any known protein motif has been identified in Buc which might interact with Vasa or vice versa. To that end, in this study, I will map Buc and Vasa binding motifs namely, Buc-Vasa binding motif (Buc-VBM) and Vasa-Buc binding motif (Vasa-BBM).

\section{Investigate the structural changes upon Buc-Vasa interaction}

From the recently published data, it is known that the LOTUS domain of Oskar interacts with RecA-like C-terminal domain of Drosphophila Vasa, and activates the Vasa ATPase activity upon interaction (Jeske et al., 2017). Consistent with this notion, I hypothesize that Buc induces zebrafish Vasa ATPase activity. Moreover, as described Buc and Oskar do not share sequence homology. Therefore, it is reasonable to postulate that both proteins perform equivalent functions adopting structural similarity. Importantly, it has been identified that eLOTUS domain of Oskar adopts a secondary structure $\alpha$ helix from its disordered state during interaction with Drosophila Vasa. Thus, I will investigate whether Buc performs the same structural changes during interaction with zebrafish Vasa.

\section{Investigate regulation of Buc-Vasa interaction in vivo}

It has been shown that the Dosophila Vasa localization to the posterior pole is dependent on Oskar protein (Breitwieser et al., 1996). Recently, it has been revealed that Oskar directly bind to Vasa and activates its ATPase activity (Jeske et al., 2017). In addition, it has been shown that Vasa mutant (vasa 4617) unable interact with Oskar as wild-type counterpart and also vasa $\Delta 617$ embryos do not show germ cells (Johnstone \& Lasko, 2004). These data suggest that the Oskar is upstream to Vasa during germ cell specification. As Buc and Oskar 
are equivalence in function, probably Buc could be upstream to Vasa during germ cell specification. Thus, it is necessary to understand regulation of Buc and Vasa interaction during germ cell specification. Therefore, after identification of Buc-VBM and Vasa-BBM, first I will investigate requirement these motifs during germ cell specification in vivo generating mutations and deletion constructs for Buc and Vasa interaction motifs. Finally, these data will facilitate to understand the hierarchy of germ cell specification pathway in zebrafish. 


\section{Results}

\subsection{Mapping the Vasa binding motif (Buc-VBM) in Buc}

Previously, we showed that Buc interacts with Vasa during the period of germ cell specification using co-immunoprecipitation. Therefore, I started to map the Buc and Vasa interaction domains using the same approach. To that end, I systematically truncated Buc and fused with GFP to the C-terminus of each fragment. Each GFP fusion fragment and fulllength of Vasa proteins were transcribed and translated in vitro followed by GFP-pull-down assay. In my results, I observed that all Buc constructs I investigated interact with Vasa suggesting the lack of specificity in Buc and Vasa interaction with in vitro GFP-pull-down assay. This interaction pattern remains the same even after I performed many optimization for example, changing washing buffer, salt concentration of buffers, detergent type and its concentration, incubation time of GFP beads with samples Therefore, I switched to bimolecular fluorescence complementation assay (BiFC) as an alternative approach to investigate Buc and Vasa interaction in vivo. The BiFC assay is based on the reconstitution of an intact fluorescent protein complex if two complementary non-fluorescent fragments are brought in close proximity by two interacting proteins upon their expression in living cells (Kerppola, 2006, 2008) (Figure 17).

A

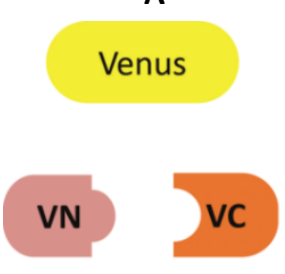

B

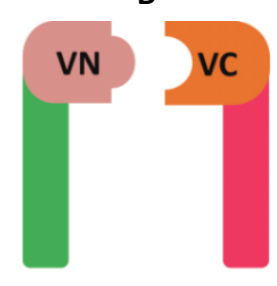

C

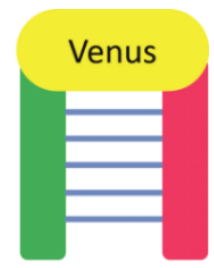

Figure 17: Schematic illustration of bimolecular fluorescence complementation assay (BiFC) assay.

(A) Venus protein (yellow) is split into two non-fluorescent parts, Venus $\mathrm{N}$-terminus (VN; rose) and Venus C-terminus (VC; orange). (B) Target proteins are fused with VN- and VC-fragments (protein A; green and protein B; pink). (C) Association of protein $A$ and $B$ (horizontal blue lines) reconstitute a functional Venus protein forming a bimolecular fluorescent complex. This image modified from (Roshan Perera and Roland Dosch, submitted 2020).

Therefore, I fused Venus protein C-terminus (VC) to the both $\mathrm{N}$-and C-terminus of Buc and Venus $\mathrm{N}$-terminus (VN) to the $\mathrm{N}$ - and C-terminus of Vasa. After co-injection of Buc and Vasa 
constructs into one-cell stage embryos, Buc-VC + Vasa-VN combination showed higher number of fluorescent positive embryos (79.4\%) (Figure 18B) while VC-Buc + VN-Vasa combination showed a smaller number of fluorescent positive embryos (48.6\%) (Figure 18E). Compared to above combinations, neither Buc-VC + VN-Vasa combination (Figure 18C) nor VC-Buc + Vasa-VN combination (Figure 18D) showed fluorescent embryos at $3 \mathrm{hpf}$. Collectively, these results suggest that the BiFC assay as a promising alternative to investigate the Buc and Vasa interaction in vivo. Besides, the results also provide some clues suggesting that the Buc and Vasa interaction motifs probably located in the C-terminal region of both proteins as the Buc-VC and Vasa-VN combination showed the highest number of fluorescent positive embryos. Therefore, I continued domain-mapping experiments fusing VC and VN fragments to the C-terminus of truncated Buc and Vasa constructs.

A

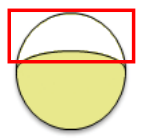

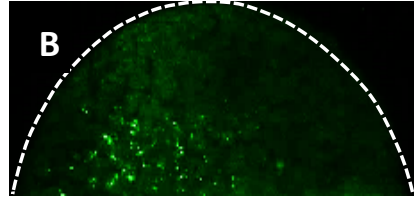
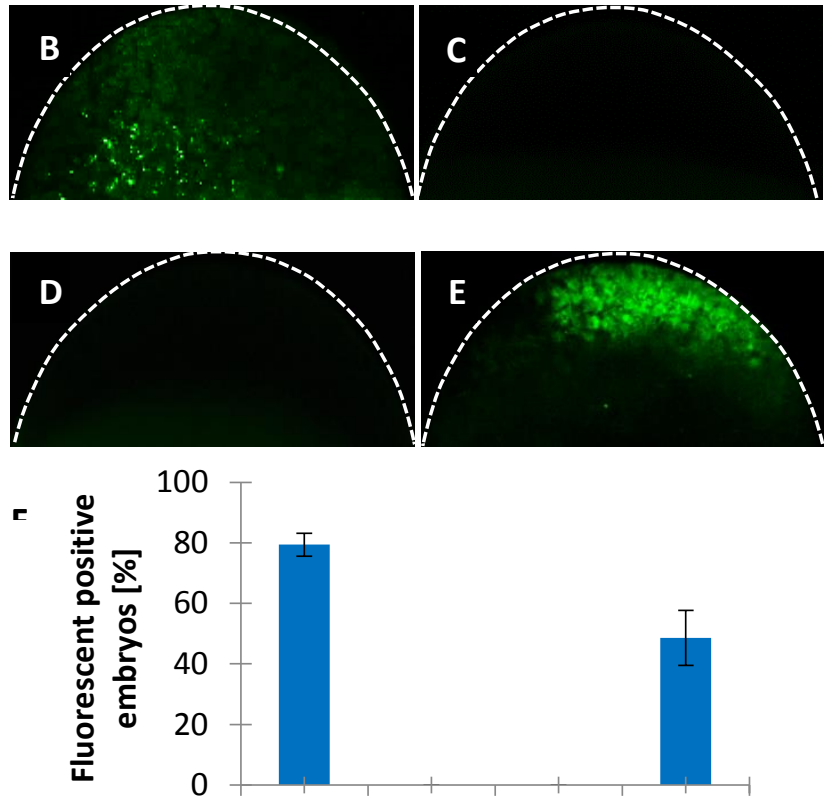

Buc-VC Buc-VC VC-Buc VC-Buc

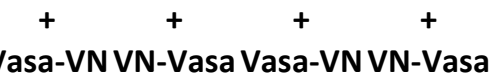

Figure 18: Buc and Vasa interact in vivo during germ cell specification.

(A) A cartoon illustrates the lateral view of embryos at $3 \mathrm{hpf}$, animal to the top. Confocal imaging area boxed in red. (B) Co-injection of Buc-VC and Vasa-VN showed fluorescent signal at the blastodisc (79.4 $\pm 3.8 \%, n=80)$. Co-injection of BucVC with VN-Vasa $(0 \pm 0 \%, n=73)$ in (C) and VC-Buc with Vasa-VN $(0 \pm 0 \%, n=80)$ in (D) did not show a fluorescent signal. (E) Co-injection of VC-Buc with VN-Vasa showed fluorescence in the blastodisc (48.9 $\pm 9.6 \%, n=66)$. (F) Quantification of fluorescent positive embryos based on the different combination of BiFC Buc and Vasa constructs. The data presented are averaged from three independent experiments. Y-axis represents percentage of fluorescent positive embryos from three independent experiments. X-axis represents injected constructs. Error bars represent standard deviation of the mean. Scale bar $100 \mu \mathrm{m}$. 
Initially, I cloned BiFC expression vectors using traditional restriction enzyme-based cloning method. This method become troublesome, if I need to clone many truncated Buc and Vasa construct during interaction domain mapping. In recent years, recombination-based Gateway ${ }^{\circledast}$ cloning vectors have been developed to investigate robust and efficient gene functions (Kwan et al., 2007; Villefranc et al., 2007; Miles \& Verkade, 2014). Therefore, to reduce the workload, I developed four Venus-BiFC Gateway adapted destination vectors (Roshan Perera and Roland Dosch submitted, 2020) (Figure. 19). These vectors are not only suitable for transcription of mRNA, but also allow to rapidly investigating interactions in tissue culture systems (Dr. Lukasz Smorag, Institute of Human Genetics, personal communication).

A

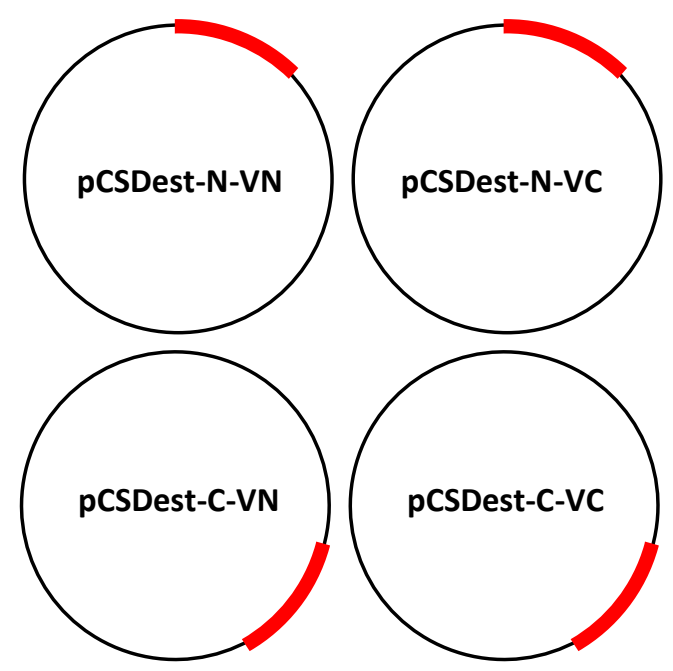

B

pCSDest-N-

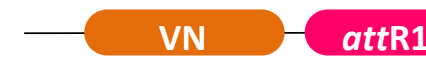

$\operatorname{ccd} B$ attR2

pCSDest-N-

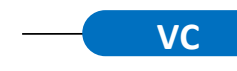

\section{attR1}

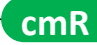

$\operatorname{ccdB}$

\section{attR2}

pCSDest-C-

\section{attR1}

$\mathrm{cm}$

ccdB

$\operatorname{attR2}$

\section{VN}

pCSDest-C-

\section{attR1}

$\mathrm{cm}$

$\operatorname{ccdB}$

$\operatorname{attR2}$

VC

Figure 19: Expression Vector maps and key core elements of BiFC gateway destination vectors.

(A) Vector maps of four different BiFC gateway destination vectors, pCSDest N-VN, pCSDest N-VC, pCSDest C-VN, pCSDest C-VC. Black line represents the vector backbone and region colored in red represent the core features of the vector described in B. (B) The vectors comprise all features of the core cassette of gateway pCSDest destination vector for recombination reaction (Villefranc et al. 2007). The core gateway cassette contains two attR sites; attR1 and attR2 (pink) flanking the bacterial toxin gene $c c d B$ (purple) for selection and the chloramphenicol resistance gene, $c m R$ (green). Venus VN (orange) and VC (blue) are fused to the N-terminus of the core cassette to generate pCSDest N-VN or pCSDest N-VC while VN and VC are fused to the C-terminus of the core cassette to generate pCSDest C-VN or pCSDest C-VC BiFC destination vectors. 
As described, Buc is predicted as an intrinsically disordered protein (Figure 16). Thus, after expressing recombinant protein in Escherichia coli, Buc is found in the insoluble pellet fraction but not in the soluble fraction. The recombinant Buc protein is highly aggregated and therefore, present in the pellet fraction as IDPs have a higher tendency to form hydrogen bonds, many electrostatic interactions via charged amino acids between proteins (Linding et al., 2004). Therefore, it is a great challenge to use full length of recombinant Buc protein to find out its structural features and biochemical functions. Alternatively, isolating a small motif, which interacts with Vasa, provides a promising approach to discover structural features and biochemical functions of Buc - Vasa interaction.

\subsection{Buc amino acid $372-394$ is the Vasa binding motif (Buc-VBM)}

To identify the region of Buc that interacts with Vasa, I performed bimolecular fluorescence complementation (BiFC) assay as described in Figure 17 and 18. There, Buc protein was systematically truncated and Venus C-terminal (VC) half was fused to the C-terminus of Buc full-length (Buc-VC) and to the truncated protein fragment (Figure 20A). The Venus $\mathrm{N}$ terminal (VN) half was fused to the C-terminus of full length of Vasa protein (Vasa-VN). At the beginning, Buc protein was divided into $\mathrm{N}$-terminal (amino acids 1-362) and C-terminal (amino acids 363-639) halves. I co-injected mRNA encoding Buc full-length, truncated Buc constructs and Vasa-VN into one-cell stage zebrafish embryos and incubated at $28.5^{\circ} \mathrm{C}$. Then embryos were imaged at $3 \mathrm{hpf}$ using fluorescence microscope. At $3 \mathrm{hpf}$, only the C-terminal region of Buc showed fluorescence signal in the blastodisc (Figure 20F) narrowing the Vasa binding motifs to amino acids 363-639 of Buc.

\subsection{Buc-VBM is highly conserved in vertebrates}

Previously, three conserved motifs of Buc have been predicted after multiple sequence alignment of vertebrate Buc orthologs (Krishnakumar et al., 2018). Of these three motifs, the central domain corresponding to amino acid 372-394, is predicted as a highly conserved domain among Buc orthologs. As the C-terminal region of Buc that I used for the BiFC assay 
encompasses the highly conserved central domain, I next examined whether this motif is involved in the interaction with Vasa.

A

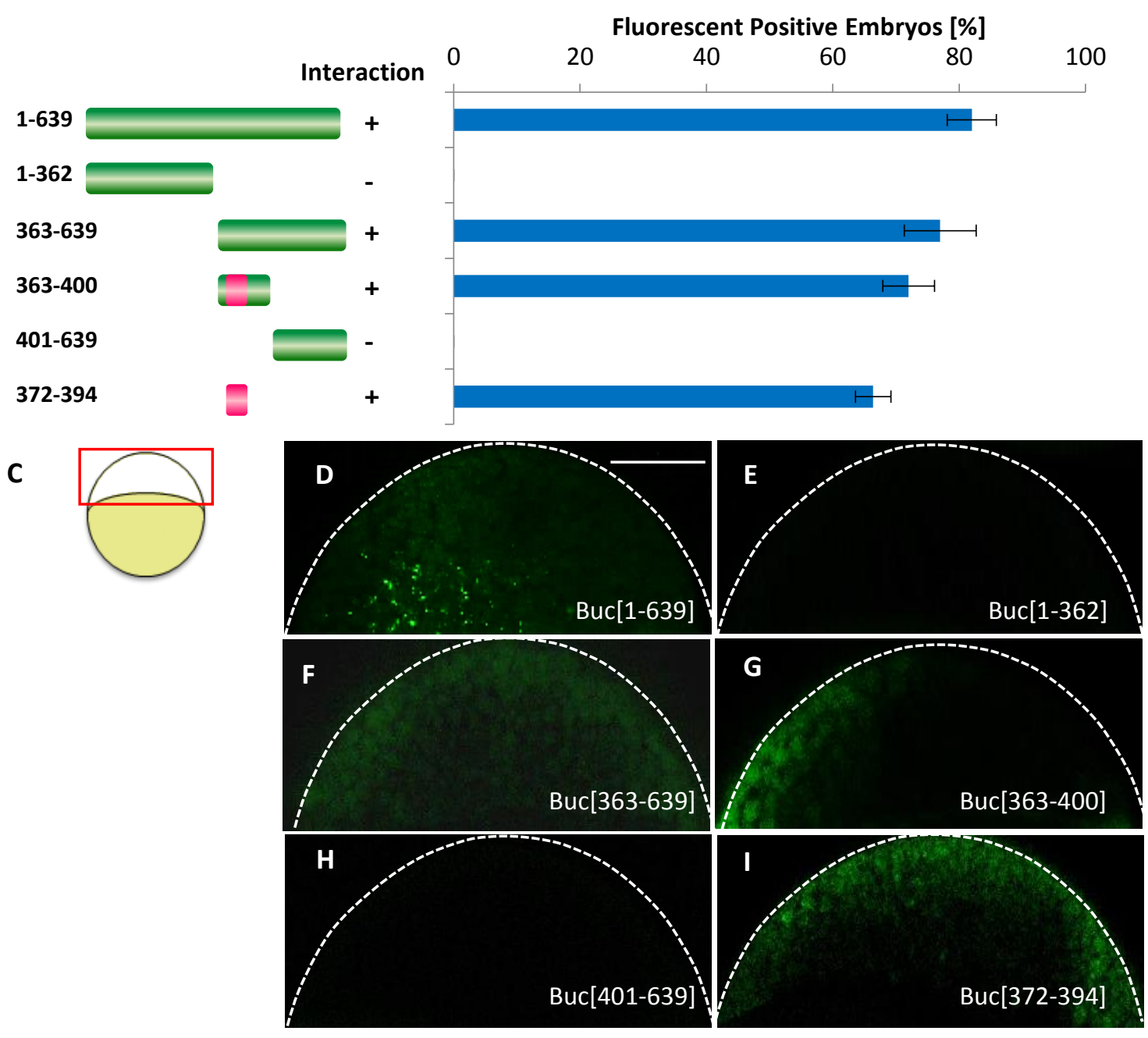

Figure 20: Identification of Buc-Vasa binding motif (Buc-VBM).

(A) Schematic illustration of systematic truncation of Buc (green). Numbers left to the colored bars indicate the corresponding amino acids. Interaction shown as ' + ', while no interaction shown as ' - '. Buc-VBM highlighted in pink. (B) Quantification of fluorescent positive embryos based on the different combination of BiFC Buc and Vasa constructs. The data presented are averaged from three independent experiments. X-axis represents the percentage of fluorescent positive embryos and Y-axis represents injected constructs. Error bars represent standard deviation of the mean. (C) A cartoon illustrates the lateral view of embryos at $3 \mathrm{hpf}$, animal to the top. Confocal imaging area boxed in red. This region is outlined with a white dashed line in panel (D - I). All Buc constructs were co-injected with wild-type(full-length??) Vasa. (D) Injection of wild-type Buc shows fluorescent signal $(82 \pm 3.9 \%, n=58)$. (E) However, Buc [1-362] and Vasa did not show fluorescent signal $(0 \pm 0 \%, n=105)$. (F) After injection, Buc [363-639] shows fluorescent signal (77 $\pm 5.7 \%, n=79)$. (G) Further, Buc [363-400] also shows fluorescence $(72 \pm 4.1 \%, n=79)$. (H) Buc [401-639] did not show a fluorescent signal $(0 \pm$ $0 \%, n=77)$. (I) Buc [372-394] shows fluorescent signal $(66 \pm 2.8 \%, n=69)$. Scale bar $100 \mu \mathrm{m}$. 
Next, I split the C-terminal region of Buc into two where the first fragment encompasses the highly conserved central domain encoding amino acid 363-400 while the second fragment bearing the remaining sequence encoding amino acid 401-639 (Figure 20A). Of these two fragments, only the fragment encoding amino acid 363-400 showed fluorescence signal in the blastodisc (Figure 20G). As this fragment bearing the highly conserved centennial (amino acid 372-394), I studied the interaction taking only the highly conserved central domain with Vasa-VN. Indeed, I observed fluorescent signal in the blastodiscs at $3 \mathrm{hpf}$ suggesting that the highly conserved central domain is sufficient to interact with Vasa (Figure 20I).

\subsection{Mapping of Buc binding motif in Vasa (Vasa-BBM)}

To investigate the Buc binding domain in Vasa, I applied the same approach as described above for Buc. Essentially, the first 277 amino acids of Vasa are predicted to be intrinsically disordered; only amino acids 278-715, which included the helicase core (HC) with the conserved Rec-A like domains, are structured. Therefore, I cloned Vasa-IDR (amino acid 1277) and Vasa HC (amino acid 278-715) fusing Venus VN half to the C-terminus of Vasa constructs (Figure 21A). After injection, at $3 \mathrm{hpf}$, I only found a positive BiFC signal using Vasa-HC with Buc but not with the Vasa-IDR (Figure 21F). These results indicate that Vasa-HC (amino acid 278-715) containing the RecA like domains interact with Buc.

It has been shown that the LOTUS domain of Oskar interacts with C-terminal RecA-like domain of Drosophila Vasa (Jeske et al., 2017). To examine whether Buc also interacts with the zebrafish Vasa RecA like domain, I divided the Vasa-HC (amino acid 278-715) into the Nterminal helicase core ( $\mathrm{N}-\mathrm{HC}$; amino acid 278-495) and the C-terminal helicase core (C-HC; amino acid 496-715). The N-HC includes the N-terminal Rec-A like domain (RecA-NTD) while the C-HC includes the Rec-A like C-terminal domain (RecA-CTD; amino acids 496-623) together with an extended sequence (denoted as ' $\mathrm{e}$ ') at the C-terminus of the RecA-CTD (eRecA-CTD; amino acids 624-715) (Figure 21A). After performing BiFC assay, I observed that the Vasa C-HC (amino acid 496-715) interacts with Buc (Figure 21H) but not the N-HC (amino 
acid 278-495) (Figure 21G). This result suggests that zebrafish Vasa RecA-like C-terminal domain interacts with Buc as Drosophila Vasa RecA-like C-terminal domain binds to the Oskar LOTUS domain.

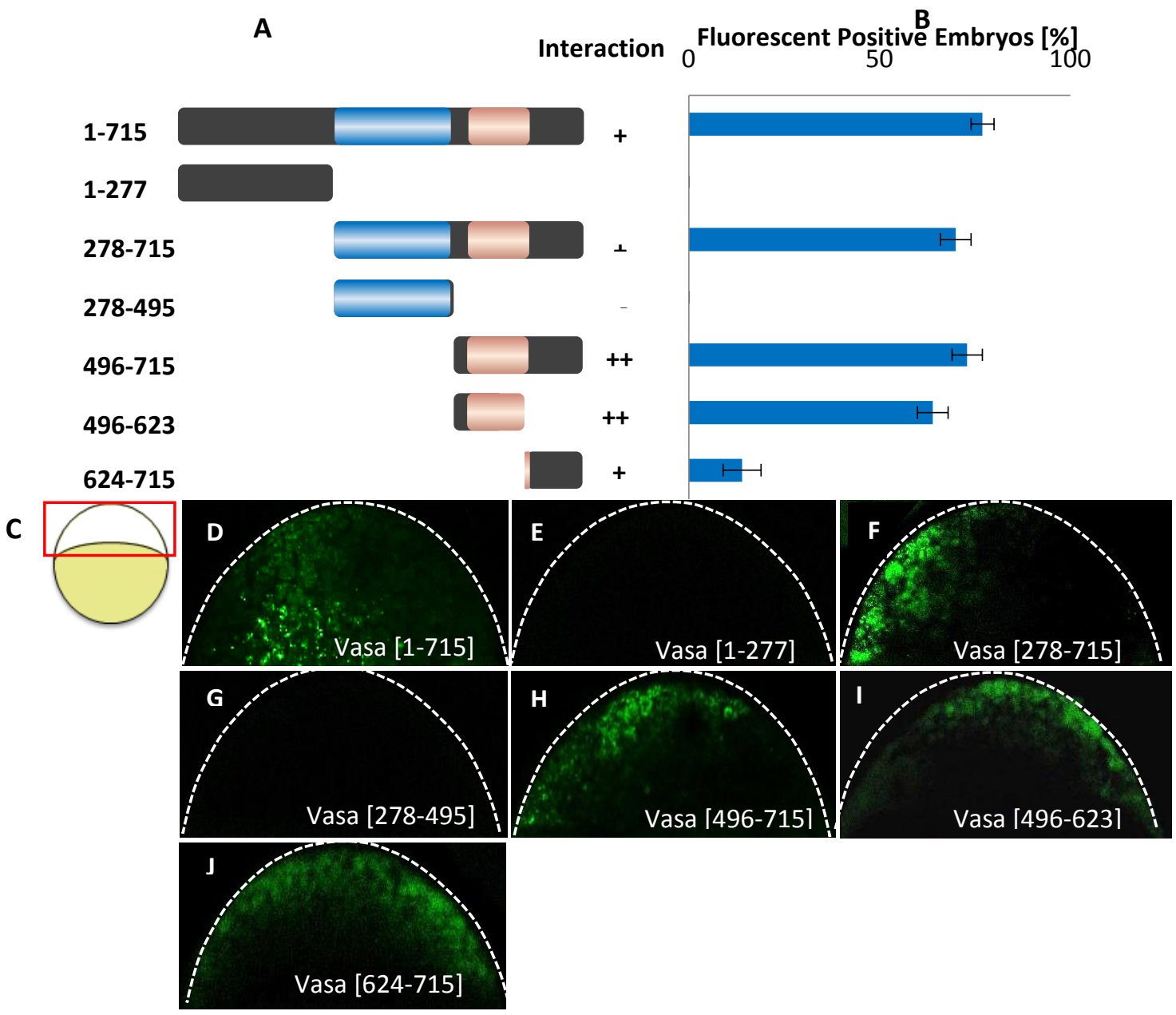

Figure 21: The Vasa-Buc binding motif (Vasa-BBM) is located in C-terminal region of Vasa.

(A) Schematic illustration of systematic truncations of Vasa (dark grey) with helicase core containing $\mathrm{N}$-terminal (blue) and C-terminal (light rose) RecA-like domains. Numbers left to the colored bars indicate the corresponding amino acids. (B) Quantification of fluorescent positive embryos based on the different combination of BiFC Buc and Vasa constructs. The data presented are averaged from three independent experiments. $X$-axis represents percentage of fluorescent positive embryos and $\mathrm{Y}$-axis represents injected constructs. Error bars represent standard deviation of the mean. Average fluorescent positive embryos $\geq 60 \%$ denoted as ' ++ ', average fluorescent positive embryos $\leq 40 \%$ as ' + ', and embryos with no fluoresce denoted as ' - '. (C) A cartoon illustrates the lateral view of embryos at $3 \mathrm{hpf}$, animal to the top. Confocal imaging area boxed in red. This region is outlined with a white dashed line in the panels D - J. (D) Injection of wild-type Vasa shows a fluorescent signal $(77 \pm 3.0 \%, n=96)$. (E) Vasa (1-277) did not show a fluorescent signal $(0 \pm 0 \%, n=43)$. (F) However, Vasa (278-715) showed fluorescence $(70 \pm 4.0 \%, n=85)$. (G) Further, Vasa (278-495) did not show a fluorescent signal $(0 \pm 0 \%, n=57)$. (H) Besides, Vasa $(496-715 ; 73 \pm 4.0 \%, n=77)$ showed fluorescent signal. (I) Vasa $(496-623 ; 64 \pm 4.0 \%$, $n=76)$ showed fluorescent signal, whereas in (J) Vasa (624-715: $14 \pm 5.0 \%, n=55)$ showed a smaller number of fluorescent embryos. Scale bar $100 \mu \mathrm{m}$. 
However, the structure solved for the LOTUS-Vasa complex revealed that the $\alpha 2$ helix of the Drosophila Vasa RecA-like C-terminal domain interfaces with $\alpha 2$ helix and $\alpha 5$ helix of the Oskar LOTUS domain during LOTUS -Vasa interaction (Jeske et al., 2017). As structural data for both Buc and Vasa are not available, I continued domain mapping using the BiFC assay to isolate a small peptide of Vasa, which is responsible for the interaction with Buc.

For this, I split the zebrafish Vasa C-terminal Helicase Core, C-HC (amino acid 496-715) into two fragments including the RecA-like C-terminal domain (amino acids 496-623) and the eRecA-CTD (amino acids 624-715) (Figure 21A). Both constructs generated fluorescent embryos upon injection with Buc in the BiFC assay. However, more fluorescent positive embryos were observed with RecA-like C-terminal domain (64\%) than with eRecA-C-terminal domain (14\%) (Figure 21B). The reason for this difference is not clear, but the Buc interaction domain in Vasa could spread over the RecA-C-terminal domain and eRecA- C-terminal domain.

I therefore changed my mapping strategy and continued by removing 50 amino acids from both the $\mathrm{N}$-and the C-terminus of C-terminal helicase core (C-HC; amino acid 496-715) (Figure 22A). With this approach, I could narrow down the Vasa-BBM to amino acid 600-665 (Figure 22A). After deleting amino acids 665 to 615 from the C-terminus of Vasa-HC, the number of fluorescent embryos dramatically dropped from $71 \%$ to $24 \%$ (Figure $A$ and $B$ ). This observation is similar to the results described above, where I observed a reduction of fluorescent embryos after splitting Vasa-Helicase Core into RecA-C-terminal domain and eRecA- C-terminal domain (Figure 21B). Taken together, these data suggest that the Bucbinding interface in Vasa is located between amino acids 600-665. 
A

B

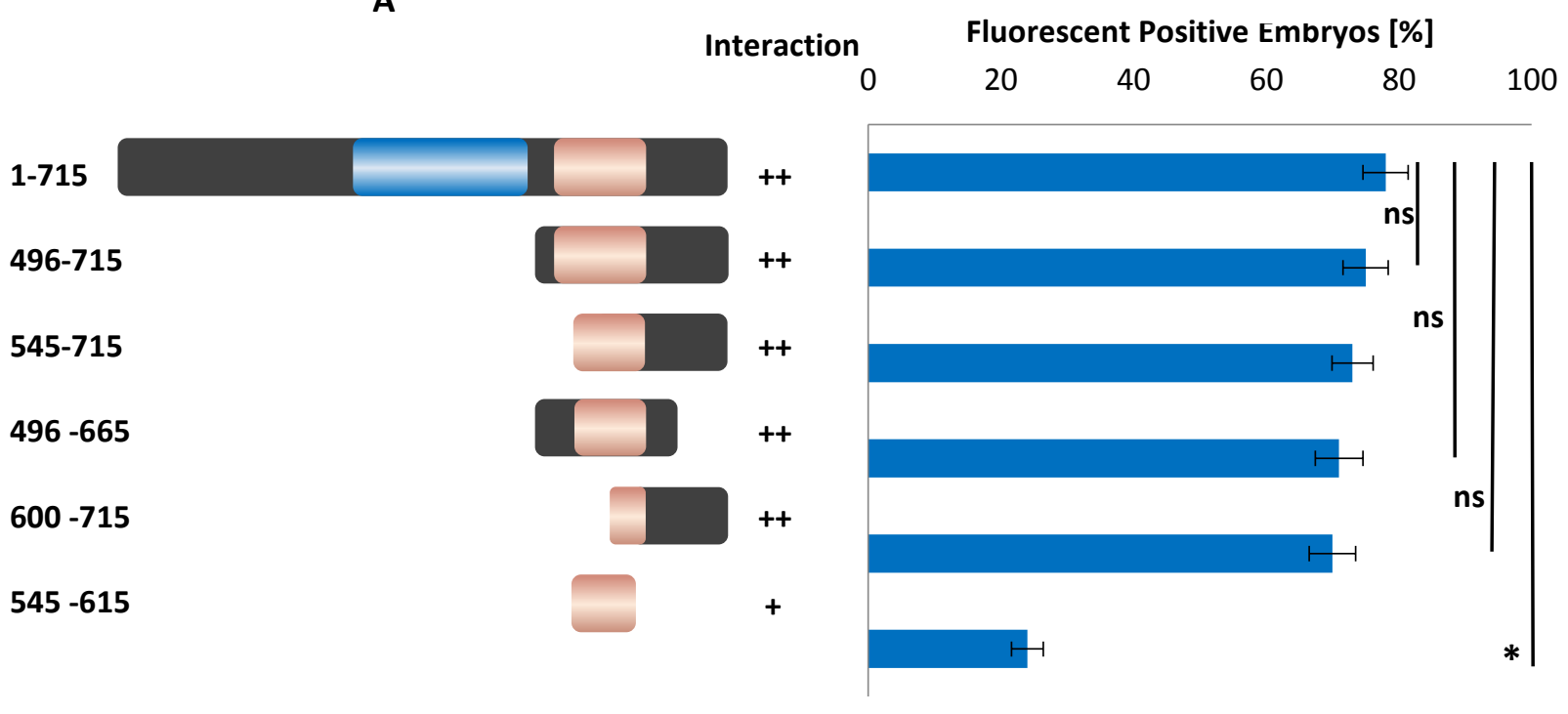

Figure 22: Vasa-BBM is potentially located in between amino acid 600-665.

(A) Schematic illustration of systematic truncations of Vasa (dark grey) with helicase core containing N-terminal (blue) and C-terminal (light rose) RecA-like domains. Numbers left to the colored bars indicate the corresponding amino acids. (B) Quantification of fluorescent embryos based on the different combination of BiFC Buc and Vasa constructs. The data presented are averaged from three independent experiments. X-axis represents percentage of average fluorescent positive embryos and Y-axis represents injected constructs. Error bars represent standard deviation of the mean. Fluorescent positive embryos $\geq 60 \%$ denoted as ' ++ ' and fluorescent positive embryos $\leq 40 \%$ shown as ' + '. Test statistics: Student's t-test, $* \mathrm{P}<0.05$. All Vasa constructs were co-injected with wild-type, full-length Buc. Wild-type Vasa (positive control: $78 \pm 3.4 \%, n=61)$, Vasa [496-715]; $(75 \pm 3.4 \%, n=85)$, Vasa [545-715]; $(73 \pm 3.1 \%, n=63)$, Vasa [496-665]; $(71 \pm 3.6 \%$, $n=58)$ and Vasa [600-665]; $(70 \pm 3.5 \%, n=58)$ constructs show $\geq 60 \%$ fluorescent embryos, while Vasa [545-615]; $(24 \pm 2.4 \%, n=53)$ shows $\leq 40 \%$ fluorescent embryos.

After I isolated the Buc interaction motif in amino acids 600-665 of Vasa, I again changed my mapping strategy to further narrow down the Buc binding site. I started to remove ten amino acids form the C-terminus of Vasa (600-665) to generate Vasa (amino acid 600-655), Vasa (amino acid 600-645), Vasa (amino acid 600-635), and Vasa (amino acid 600-625) (Figure 23A). After injection of these four constructs, I could narrow down the Buc interaction site in Vasa to a small peptide of amino acids 600-625 (Figure 23A and B). 


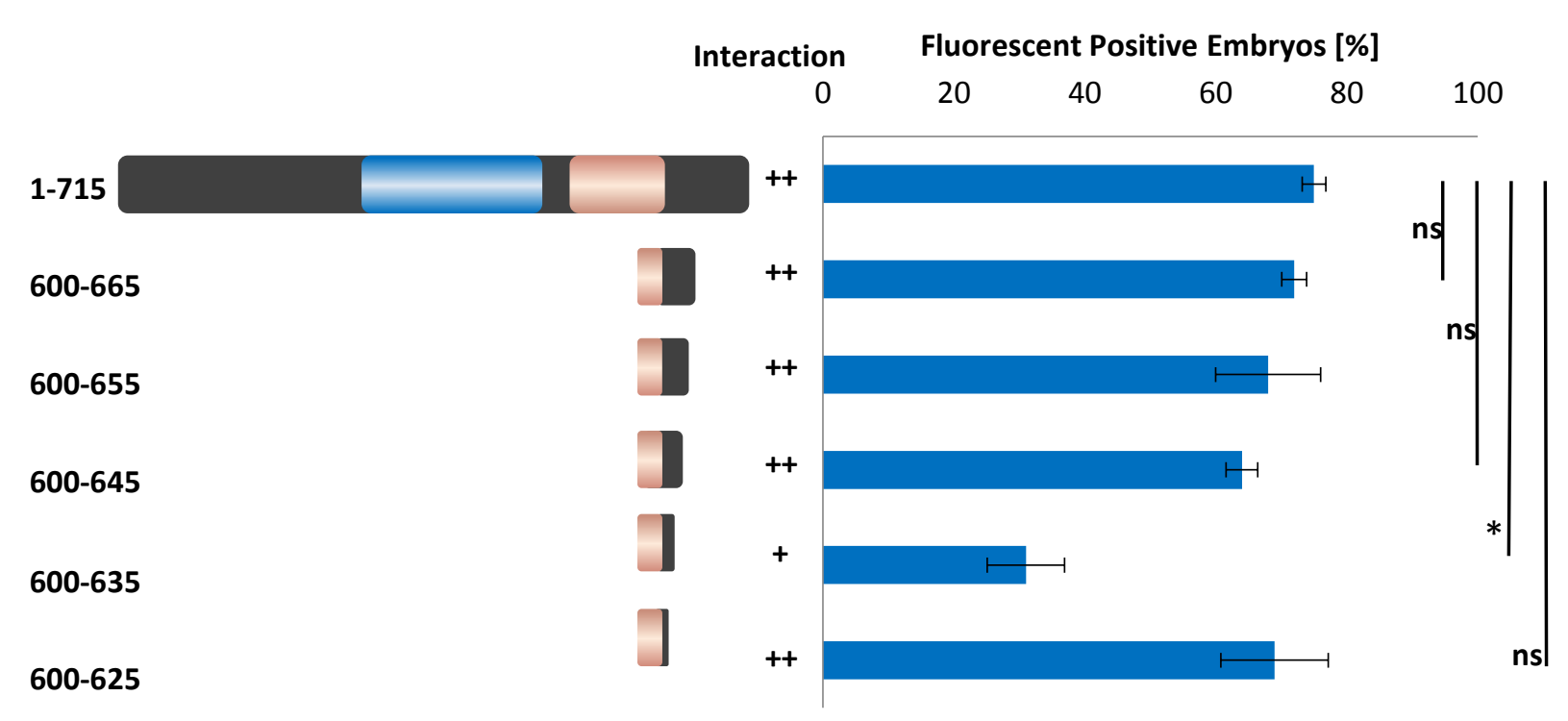

Figure 23: Vasa-BBM is located in amino acids 600-625.

(A) Schematic illustration of systematic truncation of Vasa (dark grey) with helicase core containing N-terminal (blue) and Cterminal (light rose) RecA-like domains. Numbers left to the colored bars indicate the corresponding amino acids. (B) Quantification of fluorescent positive embryos based on the different combination of BiFC Buc and Vasa constructs. The data presented are averaged from three independent experiments. The X-axis represents the percentage of fluorescent embryos and the $\mathrm{Y}$-axis shows the injected constructs. Error bars represent standard deviation of the mean. Average fluorescent positive embryos $\geq 60 \%$ denoted as ' ++ ' and average fluorescent positive embryos $\leq 40 \%$ shown as ' + '. Test statistics: Student's t-test, ${ }^{*} \mathrm{P}<0.05$. All the Vasa constructs were co-injected with wild-type Buc. There, wild-type Vasa $(75 \pm 1.8 \%, n=68)$, Vasa [600-665]; $(72 \pm 1.9 \%, n=80)$, Vasa [600-655]; $(68 \pm 8.0 \%, n=81)$ and Vasa [600-645]; $(64 \pm 2.4 \%, n=67)$ show $\geq 60 \%$ fluorescent embryos while Vasa [600-635]; $(31 \pm 5.9 \%, n=90)$ shows $\leq 40 \%$ fluorescent embryos. Interestingly, Vasa [600-625]; $(69 \pm 8.2 \%, n=64)$ shows $\geq 60 \%$ fluorescent embryos.

\subsection{Buc-VBM and Vasa-BBM are required for their interaction}

After identification of the binding motifs, I checked whether the identified Buc-VBM and Vasa-BBM are required for their interaction or whether there are still other binding motifs present in both proteins. For that, I generated a deletion construct of full-length of Buc, which lacks the amino acids 372-394 (Buc $\triangle V B M$ ), and a construct of the full-length of Vasa, which lacks the amino acid 600-625 (Vasa $\triangle B B M$ ) (Figure 24A). After injection, both deletions did not show any fluorescent signal with the wild-type binding partner in the BiFC assay (Figure 24D-F) suggesting that Buc-VBM and Vasa-BBM are essential for the Buc-Vasa interaction. 

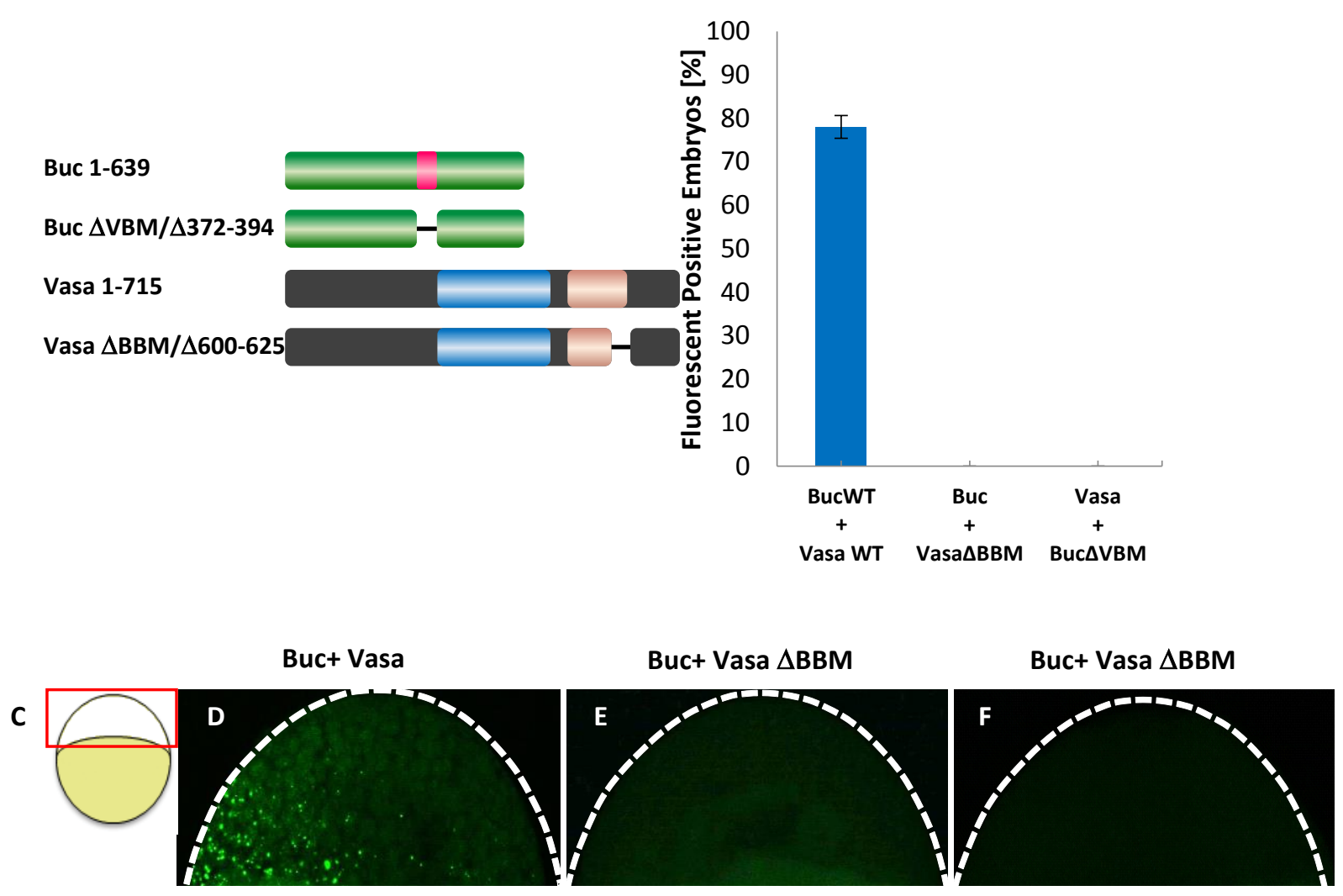

Figure 24: Buc-VBM and Vasa-BBM are required for Buc and Vasa interaction.

(A) Schematic representation of wild-type Buc, Buc $\triangle \mathrm{VBM}$, Vasa $\triangle B B M$, and wild-type Vasa. Numbers left to the colored bar represent the corresponding amino acids. (B) Quantification of fluorescent positive embryos based on the different combination of BiFC Buc and Vasa constructs. The data presented are averaged from three independent experiments. The $\mathrm{Y}$-axis represents the percentage of fluorescent embryos and the $\mathrm{X}$-axis represents the injected constructs. Error bars represent standard deviation of the mean. (C) A cartoon illustrates the lateral view of embryos at $3 \mathrm{hpf}$, animal to the top. Confocal imaging area boxed in red. This region is outlined with a white dashed line in panel (D - F). Scale bar $100 \mu \mathrm{m}$. (D) Wild-type Buc and Vasa show fluorescent positive embryos $(78 \pm 2.6, \mathrm{n}=72)$. However, wild-type Buc with Vasa $\triangle B B M$ $(0 \pm 0 \%, n=74)$. in $(E)$ and wild-type Vasa with Buc $\triangle V B M$ in $(F)$ did not show fluorescent positive embryos $(0 \pm 0 \%, n=71)$.

However, the deletion constructs might not be stable when expressed in vivo. As a specific antibody for the VC and VN fragments is not available, I fused the GFP protein to the Cterminus of all proteins to generate Buc-GFP, Buc $\triangle$ VBM-GFP, Vasa-GFP and Vasa $\triangle B B M-G F P$. I then expressed equal amount of RNA (200 ng/ $\mu$ l) in early zebrafish embryos and compared their fluorescence (Figure 25B-F). My results indicated that all the constructs are expressed in vivo and the level of fluorescence is comparable suggesting similar levels of translation and stability. Moreover, despite both Buc $\triangle \mathrm{VBM}$ and Vasa $\triangle \mathrm{BBM}$ expressed in vivo, they do 
not bind to wild-type Vasa or Buc respectively. Therefore, my findings indicate that BucVBM and VasaBBM are required for their interaction during germ cell specification in early zebrafish embryogenesis. Nevertheless, it is a compelling question whether the deletion constructs are still folding into their native conformation without the binding motifs. I therefore aimed to obtain additional structural information about the Buc and Vasa interaction to perform "minimally invasive surgery" targeting point mutations on these proteins rather deleting entire stretch of binding motifs to further characterize their interaction during germ cell specification.

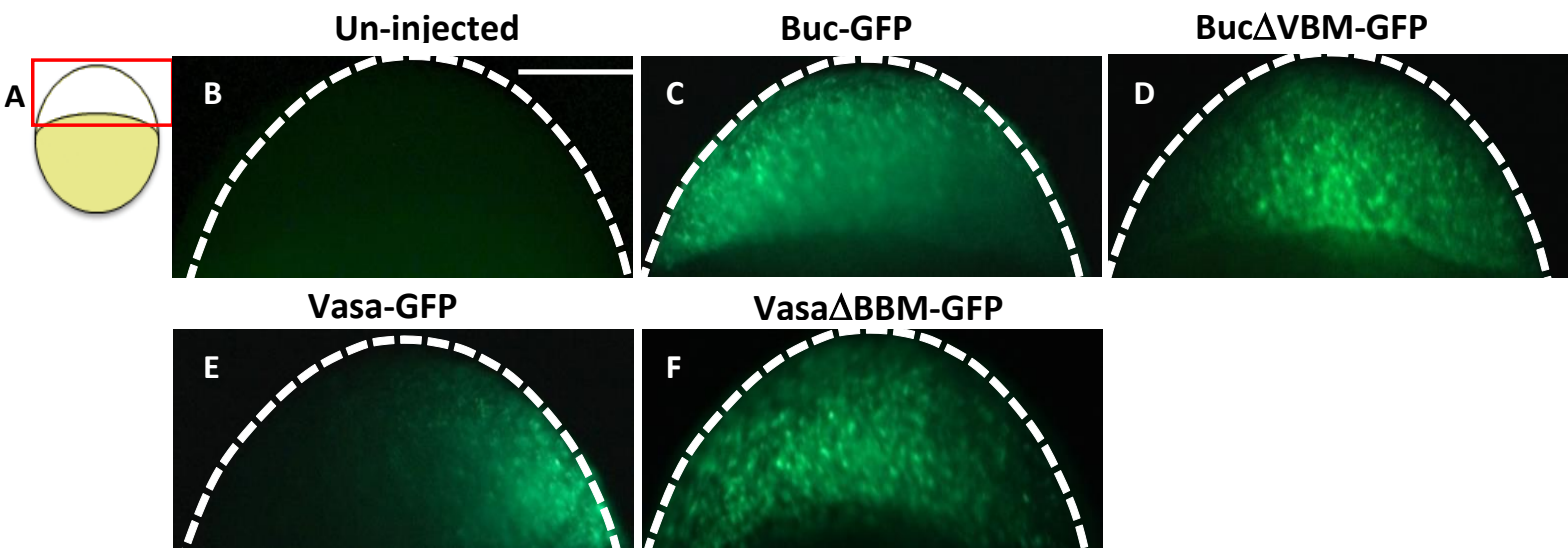

Figure 25: Buc $\triangle$ VBM-GFP and Vasa $\triangle B B M$ are expressed in vivo.

A) A cartoon illustrates the lateral view of embryos at $3 \mathrm{hpf}$, animal to the top. Confocal imaging area boxed in red. This region is outlined with a white dashed line in panel (B-F). (B-F) All the constructs; Buc-GFP, Buc $\triangle V B M-G F P$, Vasa-GFP and Vasa $\triangle B B M$ showed fluorescent signal compared to the un-injected control. Scale bar $200 \mu \mathrm{m}$

\subsection{Buc directly binds to Vasa}

With the BiFC assay, I identified the Vasa-binding motif of Buc in amino acids 372-394 and the Buc binding motif of Vasa in amino acids 600-625 using BiFC. The fluorescent signal produced in the BiFC assay is dependent on the proximity of the non-fluorescent Venus fragments suggesting that Buc and Vasa bind directly to each other. However, under in vivo conditions like I used them in my experiments with a complex mixture of proteins, the possibility exists that the Venus fragment come close to each other and emit fluorescence, although there is no direct contact between two target proteins (Miller et al., 2016). I 
therefore aimed to investigate whether Buc directly interacts with Vasa in vitro using GST pull-down assays with purified Buc and Vasa-fragments.

I fused glutathione-S-transferase (GST) to the N-terminus of the Buc-VBM (amino acid 363400). I also generated an N-terminal GST-fusion of Vasa (amino acid 227-670). After expression in E. coli, I purified both proteins by GST affinity chromatography. Purified GSTVasa treated with PreScission Protease enzyme to remove the GST-tag. After this treatment, I performed a GST pull-down assay incubating GST-Buc-VBM with Vasa. Coomassie Blue staining of the SDS-PAGE after GST-pulldown indicated that Buc-VBM directly interacts with Vasa (Figure 26). Increasing the concentration of Vasa in the binding assay did not pull-down more Vasa suggesting that the Buc and Vasa probably having a weak or transient interaction.

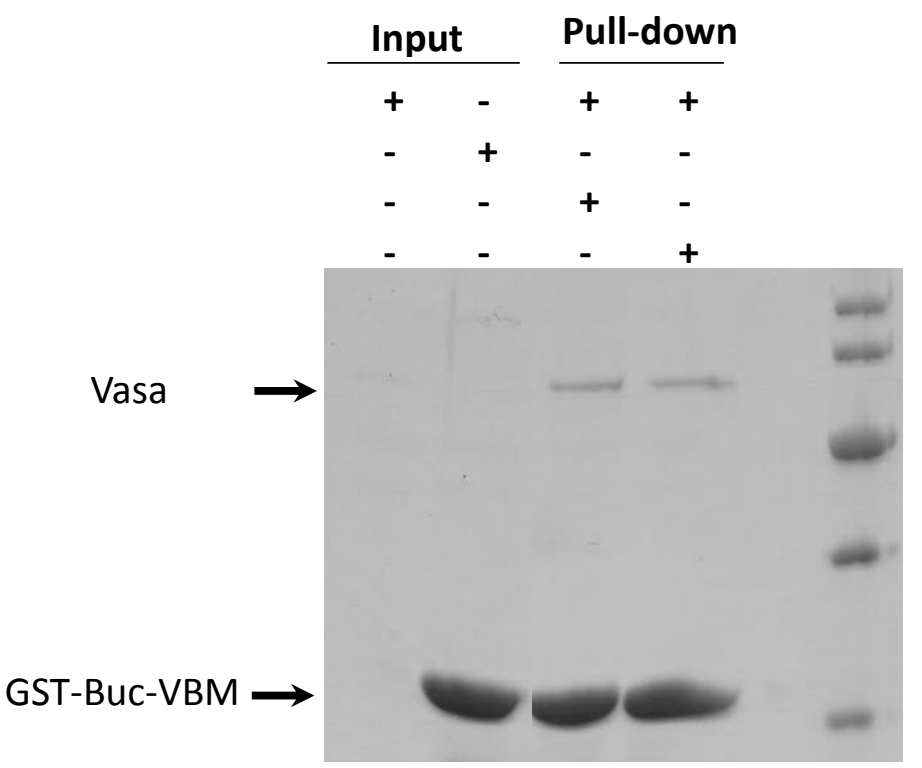

\author{
Vasa (227-670) \\ GST-Buc-VBM \\ 20 nmol Vasa \\ $30 \mathrm{nmol}$ Vasa \\ $\mathrm{kDa}$ \\ 60
}

50

40

30

Figure 26: Buc directly interacts with Vasa.

SDS-PAGE (15\%) stained with Coomassie Brilliant Blue. GST pull-down assay performed with recombinant GST-Buc-VBM (expected molecular weight approximately $32 \mathrm{kDa}$ ) and Vasa (residues 227-670; expected molecular weight approximately $56 \mathrm{kDa}$. Protein markers (in $\mathrm{kDa}$ ) are indicated on the right. In the pull-down assay, GST-Buc-VBM pulls down Vasa suggesting that both fragments directly bind to each other. Further, there was no obvious difference observed in Buc-VBM and Vasa with increasing molar concentration of Vasa. 


\subsection{Homology modeling for zebrafish Vasa}

As Drosophila Vasa and zebrafish Vasa are highly conserved in their sequence, I planned to examine the secondary structure of zebrafish Vasa using the conservation of Drosophila Vasa as a template. To that end, I aligned zebrafish Vasa encoding amino acids 227-670 which encompass the conserved helicase core with Drosophila Vasa encoding amino acids 200-623, whose crystal structure was already solved (Sengoku et al., 2006) (Figure 27). The alignment revealed that the secondary structures of zebrafish and Drosophila Vasa are highly conserved (Figure 27). More importantly, this result allowed me to perform homology modeling of zebrafish Vasa using the I-Tasser webserver ( https://zhanglab.ccmb.med.umich.edu/I-TASSER/; Roy, Kucukural and Zhang, 2010).
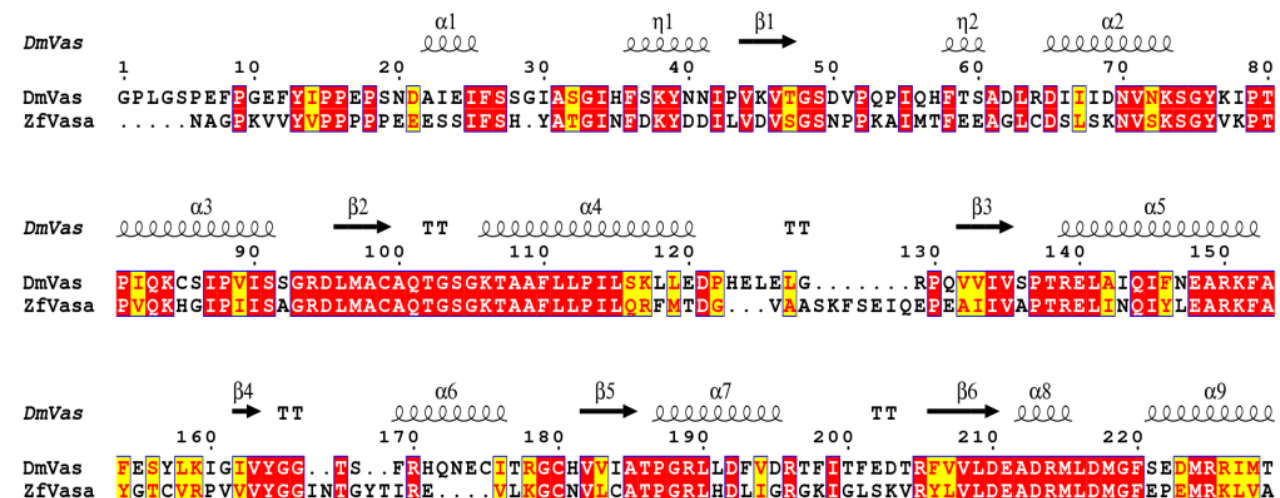

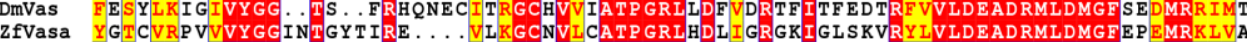
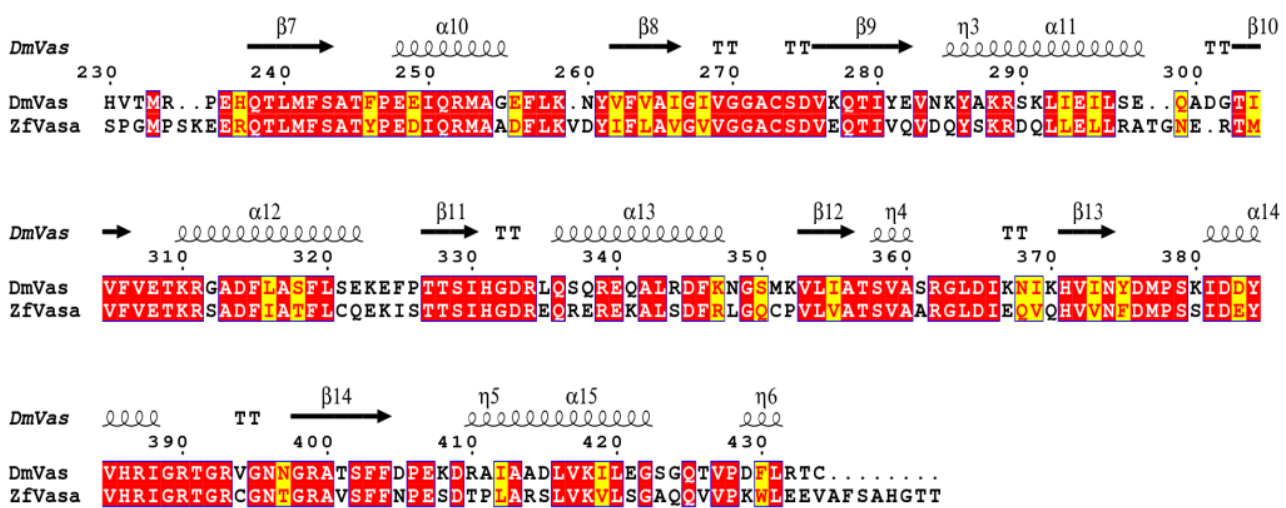

Figure 27: Pairwise amino acid sequence alignment of Drosophila and zebrafish Vasa.

Protein sequences were aligned using the T-Coffee multiple alignment server (Di Tommaso et al., 2011). For the alignment, I used partial sequences of Drosophila Vasa (DmVas; amino acids 200-623), whose structure is already solved, and zebrafish Vasa (ZfVasa; amino acids 227-670), which I used for purification. Identities are boxed in red, similarities of physico-chemical properties are boxed in yellow. Secondary structures indicated above the alignment were predicted using Espript 3.0 based on the Drosophila crystal structure PDB ID: 2DB3(Sengoku et al., 2006). Spirals indicate $\alpha$-helices and arrows indicate $\beta$-sheets. 
The I-Tasser program predicted four homology models. From these, I selected the final model with the highest C-score. The C-score calculates a confidence score as a significance of threading template alignments for the query sequence and the structure assembly. Usually, the $\mathrm{C}$-score is in the range of from -5 to 2 , where the higher $\mathrm{C}$-score predicts the best model. After homology modeling, I selected a model with a C-score of -0.87 (Table 1 ) as the final model for zebrafish Vasa. I then aligned the selected structure against the published structure for Drosophila Vasa using PyMol software (The PyMOL Molecular Graphics System, Version 2.2.3 Schrödinger, LLC and Sengoku et al., 2006). The structural alignment shows that the predicted secondary structure of zebrafish Vasa is nearly identical to Drosophila Vasa (Figure 28).

Table 1. C-score values for predicted zebrafish Vasa homology model

\begin{tabular}{|l|l|}
\hline Model & C-score \\
\hline 1 & -0.87 \\
\hline 2 & -1.02 \\
\hline 3 & -2.57 \\
\hline 4 & -3.37 \\
\hline
\end{tabular}

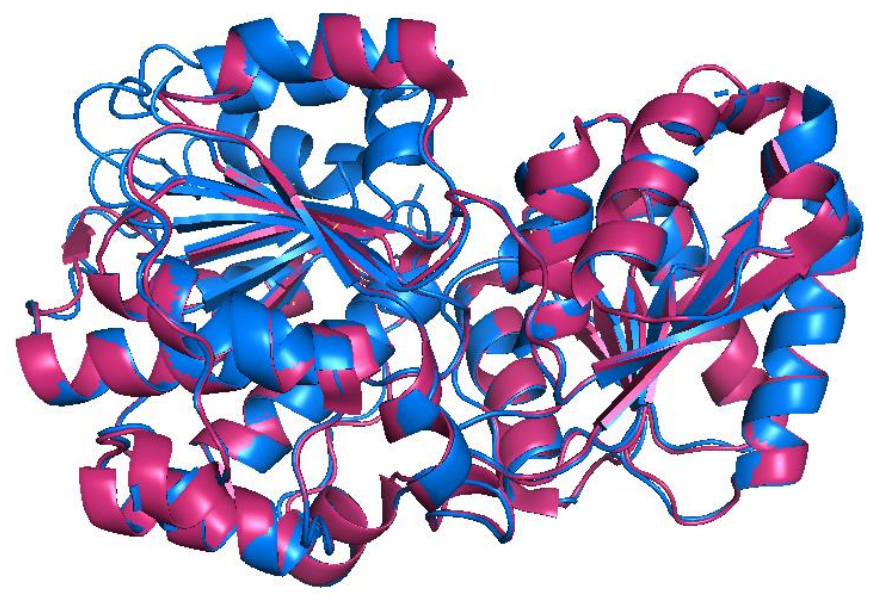

Figure 28: Alignment of a predicted zebrafish Vasa structure with the structure solved for Drosophila Vasa.

Both Drosophila Vasa (pink) and zebrafish Vasa (blue) structures were aligned using PyMol tool. The ribbon model shows that the two sequences have a nearly identical secondary structure. 


\subsection{In silico secondary structure prediction for Buc-VBM}

As mentioned previously, the amino acid composition of Buc is predicted to form an intrinsically disordered protein (Figure 16). The algorithm predicts that the Buc-VBM (amino acid 363-400) is less disordered than the rest of the protein. Previously, we showed the functional equivalence between Buc and Drosophila Oskar protein despite there is no sequence similarity between the two proteins (Krishnakumar et al., 2018). This functional equivalence could be explained by two alternative mechanisms known as convergent or divergent evolution. (Krishnakumar et al., 2018)

In the convergent evolution model, two proteins evolved independently from unrelated ancestors and by selection, constraints developed a common interactome to specify germ cells in two different species. In the divergent evolution model, two proteins evolved from a common ancestor and by selection constraints maintained a common interactome to specify germ cells (Krishnakumar et al., 2018; Wake et al., 2011).

As Oskar and Buc are essential for fertility, which is key for evolution, convergence of Buc and Oskar is less likely. However, the unrelated sequence of both proteins is supported, if both proteins form similar structures, as observed in some proteins (Bayer et al., 1998). This model therefore hypothesizes Buc and Oskar form the same structure during their interaction with Vasa in the absence of sequence homology.

Interestingly, the crystal structure for the Drosophila Oskar LOTUS-Vasa complex revealed that the $\alpha 2$ helix of the Drosophila Vas-RecA-like C-terminal domain interfaces with the $\alpha 2$ and $\alpha 5$-helix of the LOTUS domain (Jeske et al., 2017). The $\alpha 2$-helix is part of the trihelical bundle of the winged helix -turn-helix $(\mathrm{HTH})$ core of the LOTUS domain, while the $\alpha 5$-helix is found in the C-terminal region of the LOTUS domain (Figure 29). 


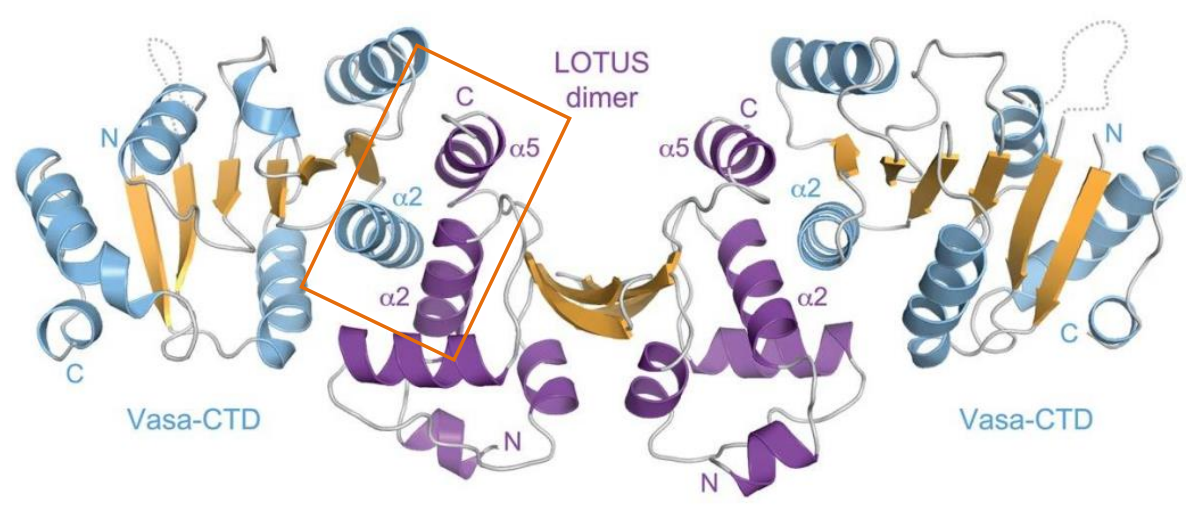

Figure 29: Illustration of Drosophila Vasa and Oskar LOTUS domain binding interface.

The LOTUS dimer of Oskar (middle) is shown in purple color and the RecA like C-terminal domain of Drosophila Vasa in light blue color. The interaction interface boxed in orange. During the interaction, the $\alpha 2$-helix of Vasa RecA like Cterminal domain interfaces with the $\alpha 2$-helix and $\alpha 5$-helix of the Oskar LOTUS domain. This image is modified from (Jeske et al., 2017).

Remarkably, the crystal structure did not show any secondary structure in the C-terminal region forming the $\alpha 5$-helix in the LOTUS-Vasa complex suggesting that this C-terminal extension of the LOTUS domain adopts an induced secondary structure upon interaction with Vasa (Jeske et al. 2017) (Figure 30).
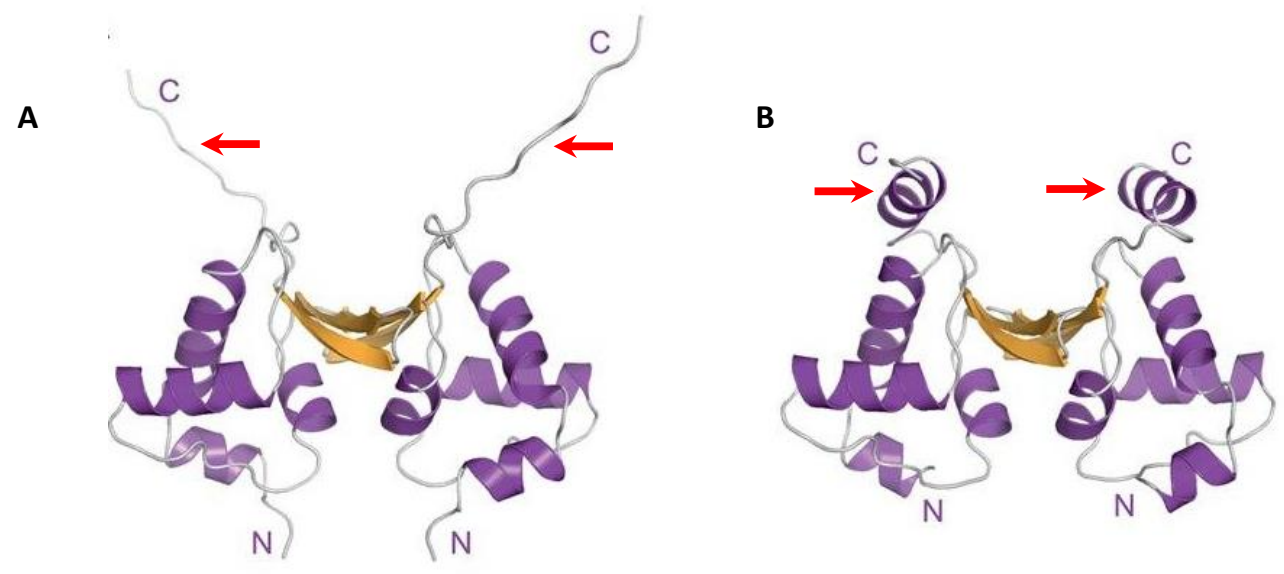

Figure 30: C-terminal extension of Drosophila Oskar LOTUS domain (eLOTUS) adopts an $\alpha$-helix during interaction with Drosophila Vasa.

(A) Apo LOTUS dimer (Protein Data Bank [PDB] 5A48) (Jeske et al., 2015). Red arrows point disordered C-terminal eLOTUS domain of each monomer. (B) After interaction with Drosophila Vasa, each monomer of eLOTUS domain adopts an $\alpha$-helix from its disordered state (red arrow). This image is modified from (Jeske et al., 2017) 
To support the hypothesis that the Buc-Vasa complex would share structural identity with Oskar-Vasa during their interaction, I performed in silico secondary structure predictions for Buc-VBM (amino acid 363-400). For this prediction, I applied three different algorithms, namely CFSSP, PEP2D, and Jnet, which are predominantly used in structural biology to predict protein secondary structures (Ashok Kumar, 2013; Singh et al., 2019; Cole et al., 2008; Drozdetskiy et al., 2015).

The CFSSP algorithm predicts $\alpha$-helices, $ß$-sheets and turns for a given protein sequence by calculating the relative occurrences of each amino acid compared to known protein secondary structures solved with X-ray crystallography (Ashok Kumar, 2013). The PEP2D algorithm is based on a random forest classification algorithm (Singh et al., 2019). For a submitted query peptide, the position-specific scoring matrix (PSSM) is calculated after aligning the given sequence with the known sequences obtained from PDB. This PSSM uses the occurrence of amino acids in secondary structures to calculate for each residue the probability values to predict $\alpha$-helices, $ß$-sheets, and turns. Inet is a sophisticated, artificial neural network (ANN) prediction algorithm (Cole et al., 2008; Drozdetskiy et al., 2015). In Inet, the query sequence is searched against UniProt reference clusters to align with position-specific iterative basic local alignment search tool (PSI-BLAST) to generate a PSSM. An HHMer program produces a probabilistic HMM profile using alignment scores. Thereafter, the Jnet algorithm uses the generated PSSM and HMM profiles to predict the final secondary structures.

When I submitted the Buc-VBM (amino acid 363-400), the three algorithms predicted variable positions for $ß$-sheets and turns (Figure 31A-C) indicating that the formation of these secondary structures is less likely. However, all algorithms independently predicted two $\alpha$-helices (Figure 31A-C), which share similar amino acids. The first $\alpha$-helix $(\alpha 1)$ consisted Glutamic acid (E), Arginine (R), Glutamine (Q) and Serine (S) (Figure 31A-C) while the second $\alpha$-helix ( $\alpha 2$ ) consisted Arginine (R), Aspartic acid (D), Glutamic acid (E) and Methionine (M) (Figure 31A-C). These results make the Vasa-binding motif in Buc an interesting object for further experiments to investigate its structure. 

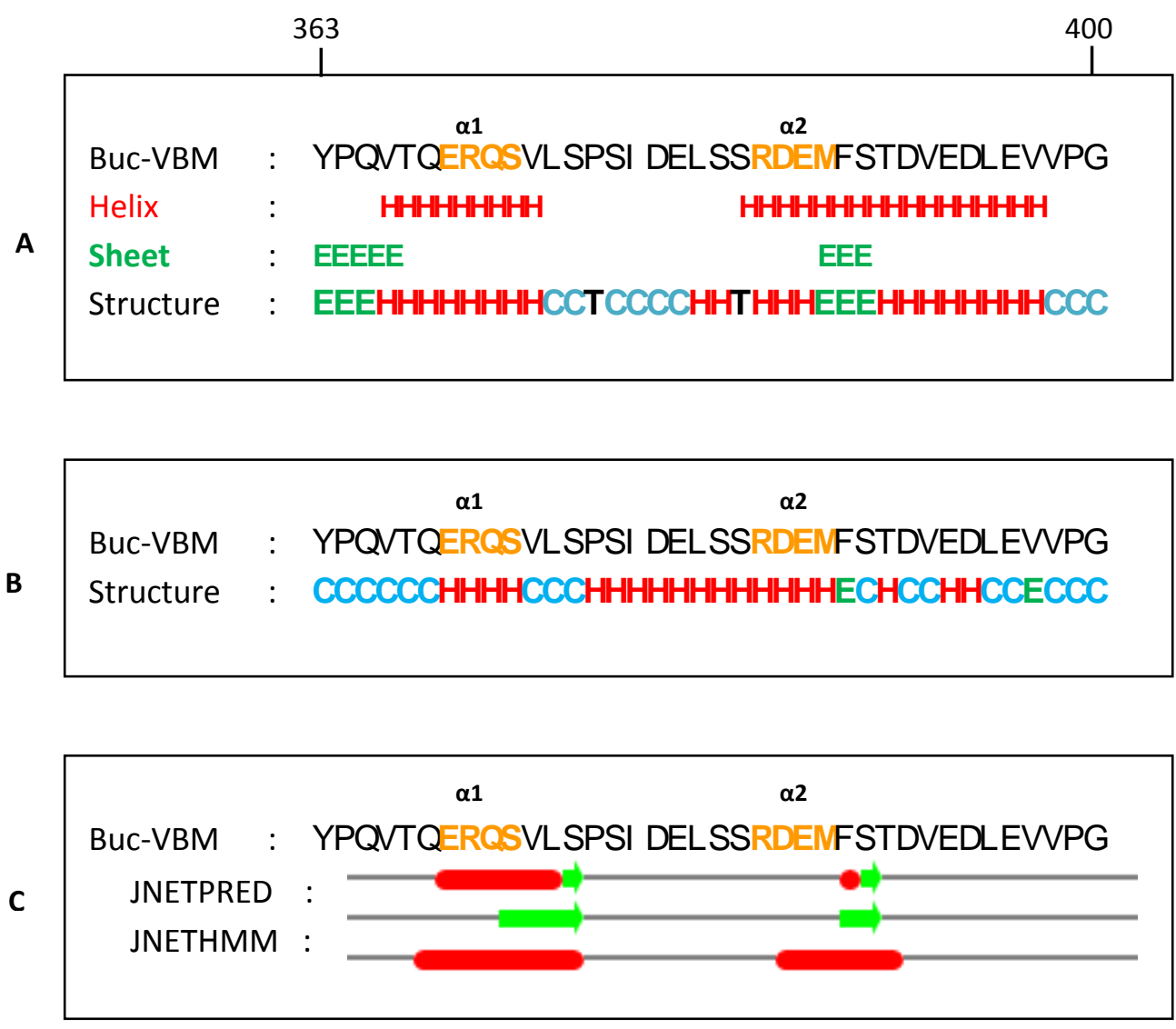

Figure 31: In silico secondary structure prediction for Buc-VBM.

In all three algorithms, predicted $\alpha$-helices denoted as ' $\mathrm{H}$ ' in red, $ß$-sheets as ' $E$ ' in green and turns as $\mathrm{T}$ in black. The consensus secondary structure is shown at the bottom. Note that random coils in the consensus structure are denoted as ' $C$ ' in light blue. The first (363) and last (400) amino acid sequence of the Buc-VBM is displayed on top of the sequence of panel A, which is common for the panel B and C. (A) Secondary structure prediction using the CFSSP algorithm. The algorithm predicted two $\alpha$-helices, $\alpha 1$ and $\alpha 2$. The $\alpha 1$ contains amino acid ERQS (orange) and $\alpha 2$ contains amino acid RDEM (orange). It also predicted two ß-sheets, which contains amino acid YPQ from the N-terminus and MFS from the Cterminus. (B) Secondary structure prediction using PEP2D algorithm. The Buc-VBM amino acid sequence is displayed on top. The algorithm predicted two $\alpha$-helices, $\alpha 1$ and $\alpha 2$, which shares the same amino acids predicted by CFSSP algorithm. (C) Secondary structure prediction using the Jnet algorithm. Buc-VBM sequence is displayed on top. The algorithm predicted two $\alpha$-helices, $\alpha 1$ and $\alpha 2$, which shares the same amino acids predicted by CFSSP algorithm and Jnetpred program within the Jnet algorithm predicted two ß-sheets (green box; amino acid RQSV from the N-terminus and EMF from the C-terminus). JnetHMM shows the prediction of $\alpha$-helices by the HMMER profile network in the Jnet algorithm. 


\subsection{Buc-VBM adopts $\alpha$-helices from its disordered state}

To confirm the in silico predicted secondary structures of Buc-VBM experimentally, I applied circular dichroism (CD) spectroscopy. CD-spectroscopy uses light absorption to measure the difference in absorbance of right- and left-handed circular-polarized light when it passes through optically active molecules (Norma J. Greenfield, 2012). Predominantly, amide groups of the polypeptide backbone are optically active and their CD spectrum changes with the local conformation of a secondary structure in the protein.

Structural elements such as $\alpha$-helices, $\beta$-sheets, $\beta$-turn, and random coils have characteristic CD spectra over corresponding wavelength $\alpha$-helices show negative CD spectra at $222 \mathrm{~nm}$ and $208 \mathrm{~nm}$ and positive spectra at $193 \mathrm{~nm}$. Proteins with antiparallel $\beta$ - sheets exhibit negative spectra at $218 \mathrm{~nm}$ and positive bands at $195 \mathrm{~nm}$. Disordered proteins have considerably very low CD above $210 \mathrm{~nm}$ and negative spectra near $195 \mathrm{~nm}$ (Figure 32A). (Norma J. Greenfield, 2012).

To perform CD-spectroscopy, I used purified Buc-VBM (amino acid 363-400) after removing the GST moiety by treatment with PreScission Protease. Next, I rebuffered the protein by dialysis to provide appropriate conditions for CD-spectroscopy. At native, aqueous conditions and neutral $\mathrm{pH}, \mathrm{I}$ calculated the molar ellipticity $\left([\theta] \mathrm{deg} \times \mathrm{cm}^{2} / \mathrm{dmol}\right)$ for Buc-VBM. It showed a strong negative molar ellipticity at $200 \mathrm{~nm}$, low negative molar ellipticity at 210$230 \mathrm{~nm}$, and $190 \mathrm{~nm}$ (Figure 31B). Therefore, the CD profile of Buc-VBM is typical for disordered proteins, which lack any secondary structure (Fig. 32A).

To assess whether a disordered protein has the propensity to form stable secondary structures, the organic solvent trifluoroethanol (TFE) with its crowding properties has been used to induce secondary structures in short peptides (Laureto et al., 2001). TFE disrupts hydrogen bond formation between water molecules in the solvent shell and the amide proton of the peptide backbone. Consequently, intramolecular hydrogen bond formation is strengthened and the stability of secondary structures increases (Walgers et al., 1998; 
Kaczka et al., 2014). In the presence of $30 \%$ as well as 50\% TFE, I observed that Buc-VBM displays the typical CD spectra for $\alpha$-helices (Figure 32B). These results suggest that Buc-VBM is prone to adopt an ordered conformation from its disordered nature, when it loses its hydration shell as it occurs during protein-protein interaction. Together, my in silico secondary structure predictions and CD-spectroscopy data provide strong support for the hypothesis that the Buc-Vasa interaction show structural similarities to the binding of the eLOTUS domain of Oskar with Drosophila Vasa RecA-like C-terminal domain.

A

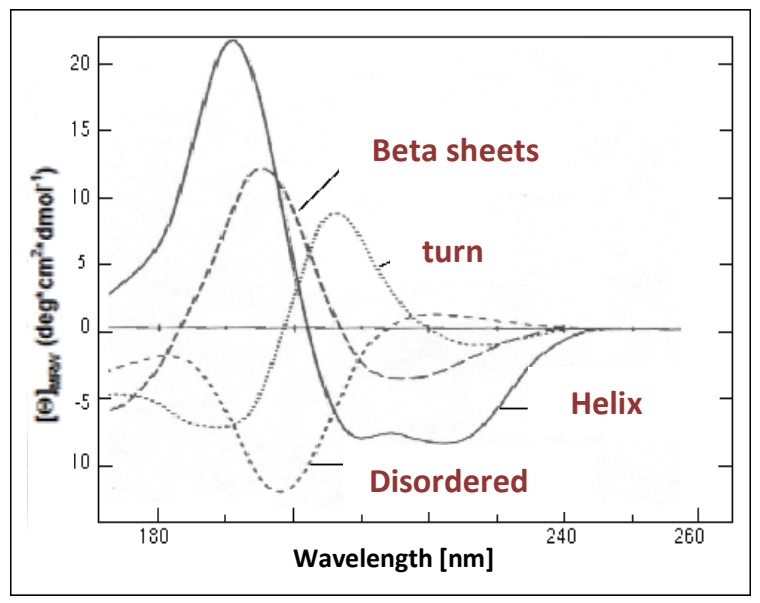

B

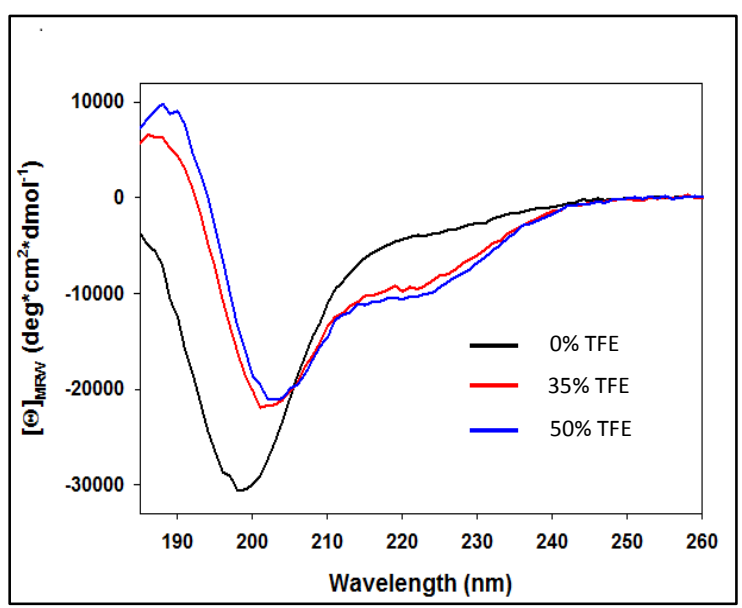

Figure 32: Secondary structure prediction for Buc-VBM (amino acid 363-400) using CD-spectroscopy.

The $\mathrm{Y}$-axis represents the molar ellipticity $([\theta])$ which is $C D$ corrected for concentration. The $\mathrm{X}$-axis represents the corresponding wavelength. (A) A representation of reference CD-spectra (Norma J. Greenfield, 2012) CD-spectra for characteristic secondary structures are labelled. $\alpha$-helices show negative CD spectra at $222 \mathrm{~nm}$ and $208 \mathrm{~nm}$ and positive spectra at $193 \mathrm{~nm}$. Proteins with antiparallel $\beta$ - sheets exhibit negative spectra at $218 \mathrm{~nm}$ and positive bands at $195 \mathrm{~nm}$. Disordered proteins have very low CD spectra above $210 \mathrm{~nm}$ and negative spectra near $195 \mathrm{~nm}$. (B) Represents the CDspectra for Buc-VBM. In the native aqueous conditions and neutral pH, the CD spectra calculated for Buc-VBM was typical for a disordered protein (black line). After addition of different concentration of TFE (35\%-red line and 50\%-blue line), BucVBM shows CD-spectra characteristic for $\alpha$-helices from its disordered state with the TFE crowding agent. 


\subsection{Buc-VBM is a novel activator of zebrafish Vasa helicase activity}

My data show that Buc directly binds to Vasa, but also raise the question what are the biochemical consequences of this interaction. It has been previously shown that the helicase activity of DEAD box proteins is modulated by protein cofactors. Fascinatingly, it has been discovered that the Oskar LOTUS domain stimulates the ATPase activity of Drosophila Vasa upon their interaction albeit Oskar is not belonging to a canonical protein family of putative helicase regulating cofactors (Jeske et al., 2017). Therefore, I next aimed to check whether Buc-VBM could modulate the ATPase activity of zebrafish Vasa during their interaction. To quantify the Vasa activity, I used an in vitro NADH/LDH coupled ATPase assay (Kiianitsa et al., 2003).

In principle, this assay monitors the reduction of NADH absorbance at $340 \mathrm{~nm}$, which is directly proportionate to the rate of ATP hydrolysis. For each cycle of ATP hydrolysis by RNA helicases, phosphoenolpyruvate (PEP) is converted to pyruvate by pyruvate kinase (PK), which is coupled to the regeneration of ATP. Subsequently, pyruvate is reduced to lactate by lactate dehydrogenase (LDH) while oxidizing $\mathrm{NADH}$ to $\mathrm{NAD+}$, which I measured at $340 \mathrm{~nm}$. (Figure 33). 


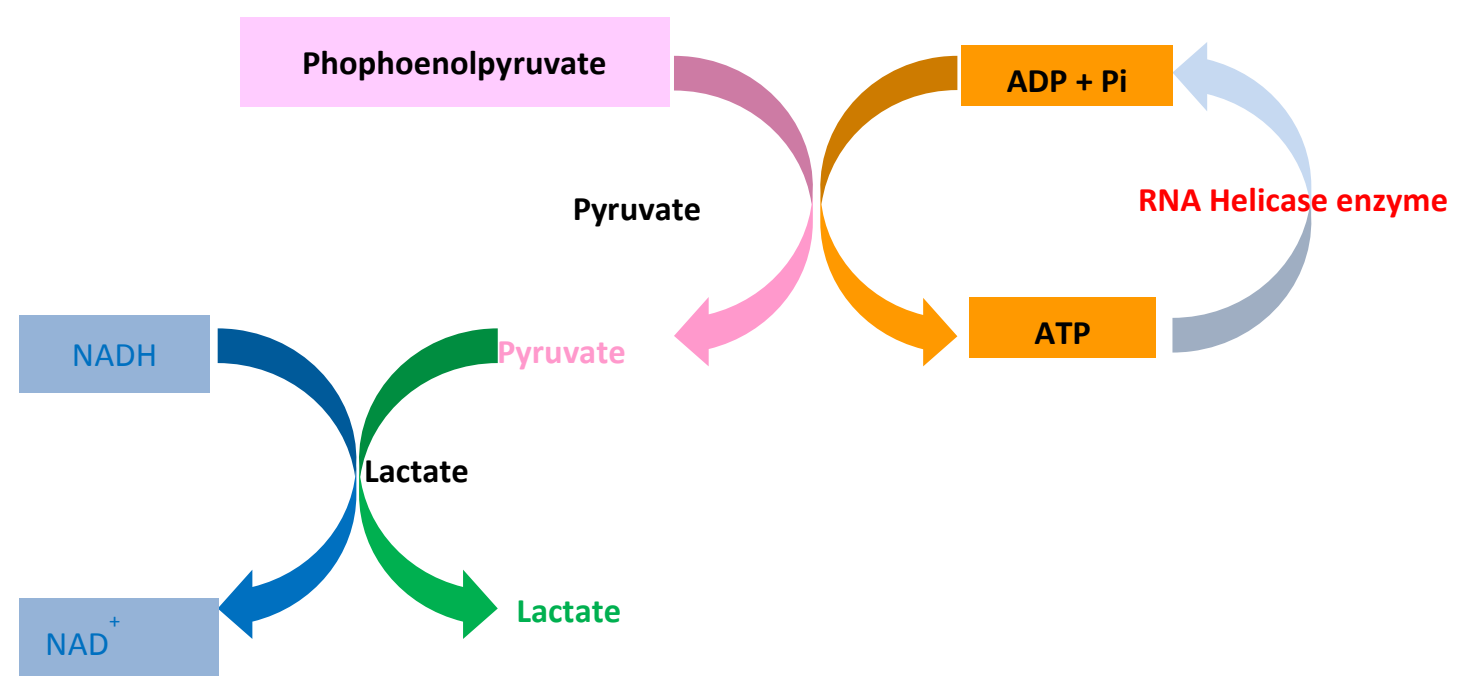

Figure 33: Schematic representation of NADH/LDH coupled ATPase assay.

This assay monitors the reduction of NADH absorbance at $340 \mathrm{~nm}$, which is directly proportional to the rate of ATP hydrolysis. For that, substrates such as Phophoenolpyruvate (pink box), NADH (light blue box) ATP (orange box) are mixed with pyruvate kinase/lactate dehydrogenase enzyme mixture. For each cycle of ATP hydrolysis by RNA helicases, phosphoenolpyruvate is converted to pyruvate by pyruvate kinase, which is coupled to the regeneration of ATP. Consequently, pyruvate is reduced to lactate by lactate dehydrogenase (LDH) while oxidizing NADH to NAD+, which measured at $340 \mathrm{~nm}$.

In control experiments, I observed a mild ATPase activity after incubating $5 \mu \mathrm{M}$ Vasa together with $2.5 \mathrm{mM}$ ATP compared to samples incubated with only $100 \mu \mathrm{M}$ Buc (Figure 34). Remarkably, ATPase activity was strongly induced when I added $100 \mu \mathrm{M}$ Buc-VBM to Vasa. These results suggest that Buc-VBM functions as a novel cofactor, which stimulates the ATPase activity of zebrafish Vasa (Figure 34). More importantly, this discovery reveals for the first time a biochemical function for the Buc protein during germ cell specification. 


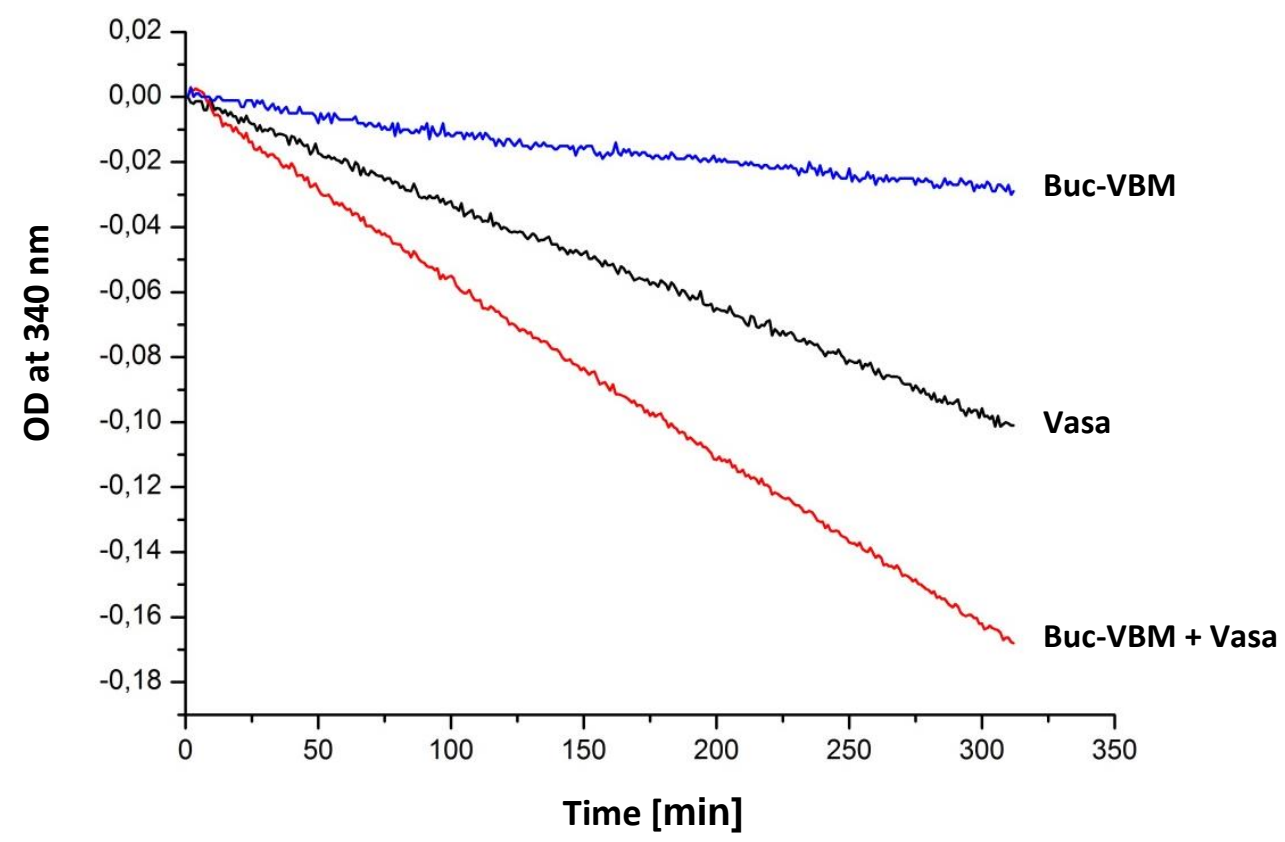

Figure 34: Buc-VBM activates Vasa helicase activity.

Y-axis denotes the reduction of NADH absorption, which is directly proportional to the rate of ATP hydrolysis. The absorbance was measured at $340 \mathrm{~nm}$. X-axis denotes the incubation time in minutes. The data presented are averaged from three independent experiments. Incubation of $100 \mu \mathrm{M}$ Buc-VBM with $2.5 \mathrm{mM}$ ATP showed very mild ATPase activity (Negative control, blue line). A higher background ATPase activity was observed after incubation of $5 \mu \mathrm{M}$ Vasa with $2.5 \mathrm{mM}$ ATP (black line). Fascinatingly, ATPase activity was approximately doubled when $100 \mu \mathrm{M}$ Buc-VBM was added to $5 \mu \mathrm{M}$ Vasa, and $2.5 \mathrm{mM}$ ATP. Data sheet is attached in the appendix I.

\subsection{Buc-Vasa binding motifs and intrinsically disordered region is not sufficient for germ cell formation}

Previously, we showed that wild-type Buc and Vasa induce ectopic germ cells when inject into a somatic cell in 16-cell stage of embryos. With this assay, I wanted to check whether the Buc-VBM or Vasa-BBM is sufficient to induce ectopic germ cells. To test for germ cell induction, I injected mRNA of wild-type Buc, Buc-VBM and Vasa-BBM into a somatic cell of 16-cell stage embryos. In my results, except wild-type Buc, Buc-VBM and Vasa-BBM did not show ectopic germ cells at $15-18$ somite stage embryos (Figure 35A-C). It has been shown that Buc, Oskar, and Vasa are intrinsically disordered proteins. Therefore, these proteins aggregate through the IDRs. Thus, it is arguable that the induction of ectopic germ cells is dependent on IDR of the proteins in addition to Buc and Vasa binding motifs. To test this, in the same assay I injected mRNA of mutant Buc encoding amino acids 1-601 (BucP ${ }^{106}$ ), which 
lacks the terminal 38 amino acids yet retains most of the IDRs. As a control, I injected mRNA of an unrelated protein called human fused in sarcoma (hFUS), which is strongly intrinsically disordered, into a somatic cell of a 16-cell stage zebrafish embryo. In my results, neither hFUS (Figure 35D) nor BucP ${ }^{106}$ did induce ectopic germ cells (Figure 35E), even though Buc $^{\mathrm{p} 106}$ contains the Buc-VBM (amino acid 363-400). Collectively, these results postulate that neither aggregation of these proteins via their IDRs nor interaction through their binding motifs are sufficient to form ectopic germ cells.

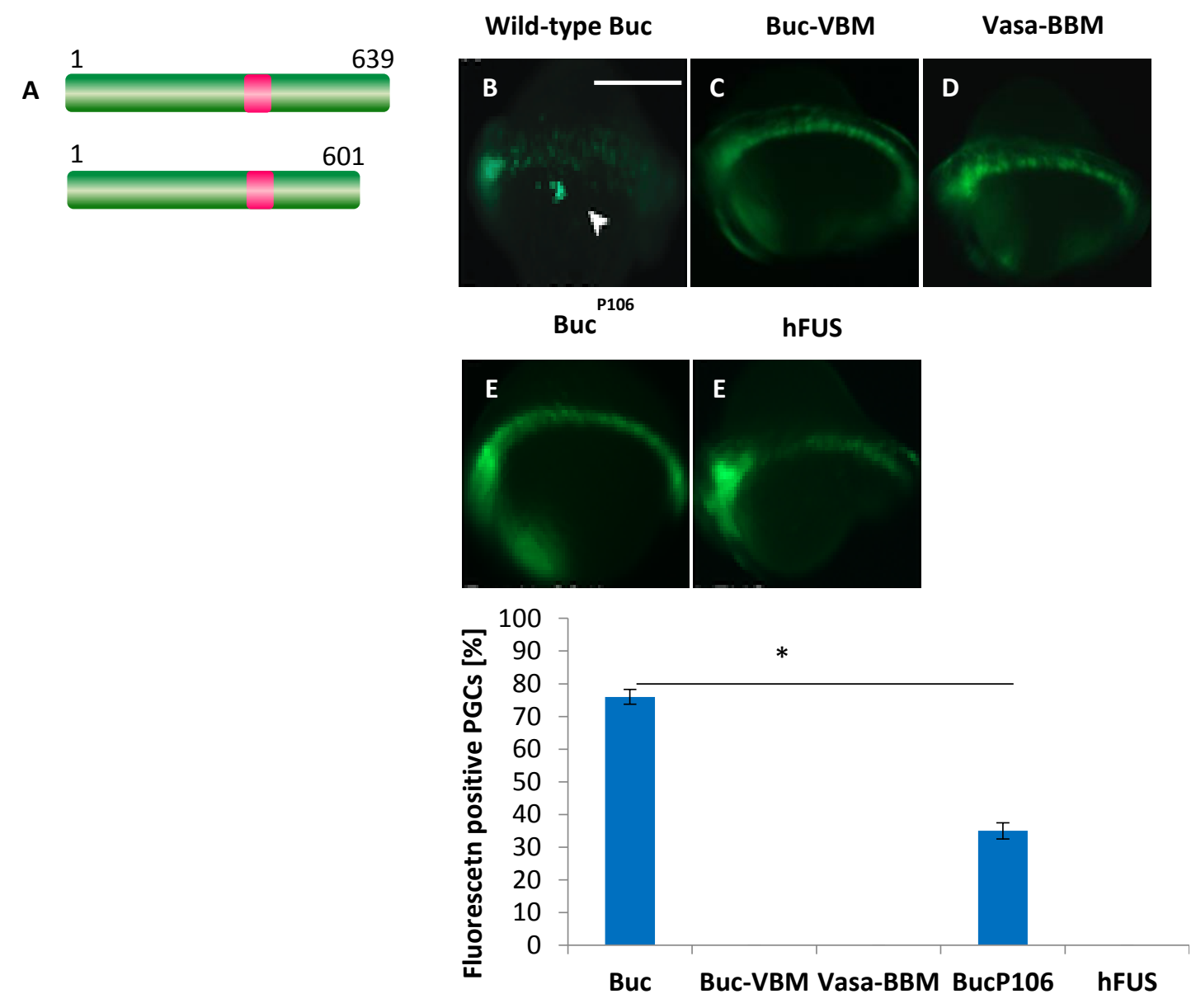

Figure 35: Buc-Vasa binding motifs and its intrinsically disordered regions are not sufficient for germ cell formation.

(A) A cartoon representing wild-type Buc (top) and mutant BucP ${ }^{106}$ (bottom). Reporter RNA, nanos3-3'-UTR GFP and all the other RNAs are injected into a somatic cell (corner cell) of a 16-cell stage embryo. (B) Wild-type Buc (amino acid 1-639) induced ectopic germ cells (76.6 $\pm 2.3 \% ; n=60$ ) (C) Buc-VBM (amino acid 363-400;0 $00 \%, n=26$ ), (D) Vasa-BBM (amino acid $600-625 ; 0 \pm 0 \%, n=35$ ), (E) mutant BucP ${ }^{106}$ (amino acid 1-601; 35.9 $\pm 2.6 \% ; n=60$ ) and (F) hFUS did not induce ectopic germ cells. (F) Quantification of fluorescent positive PGCs. The data represent three independent experiments. The Y-axis shows the percentage of fluorescent positive PGCs containing embryos and the X-axis indicates the injected constructs. Error bars represent standard deviation of the mean. Test statistics: Student's t-test. ${ }^{*} \mathrm{P}<0.05$. Scale bar $100 \mu \mathrm{m}$. Note that, I performed the experiments related to figure 35B,E and $\mathrm{F}$ and published in (Krishnakumar et al., 2018). 


\subsection{Buc-VBM and Vasa-BBM act as dominant negative proteins during germ cell specification}

Unexpectedly, the injection of mutant Bucp106 (aa1-601) with the VBM does not induce the formation of germ cells (Fig. 35). This result raises the hypothesis that a second interaction of Buc might be required to specify germ cells in addition to having IDRs and Buc and Vasa binding motifs. As a result of this hypothesis, I further speculated that the Buc-VBM acts as a dominant negative protein by inhibiting endogenous Vasa and thus PGC specification. To test this, I injected mRNA of Buc-VBM into one-cell embryos of Buc-GFP transgenic fish. Compared to un-injected controls, I observed a reduction in the number of germ cells in embryos injected with Buc-VBM (Figure 36B and C)

A

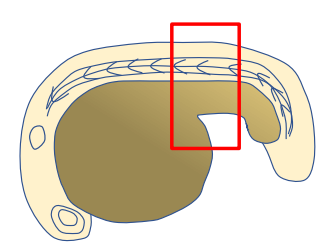

B

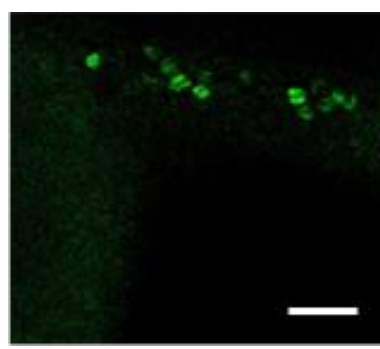

C
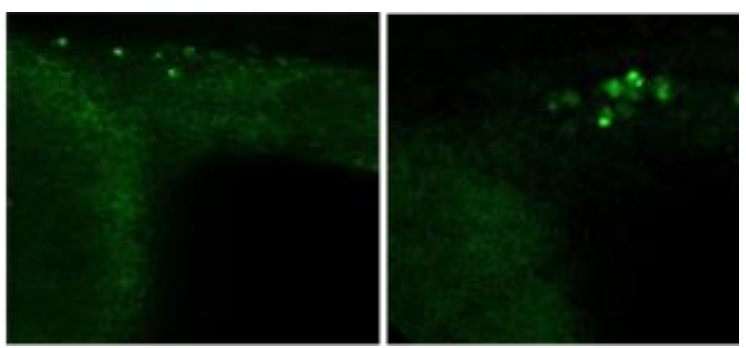

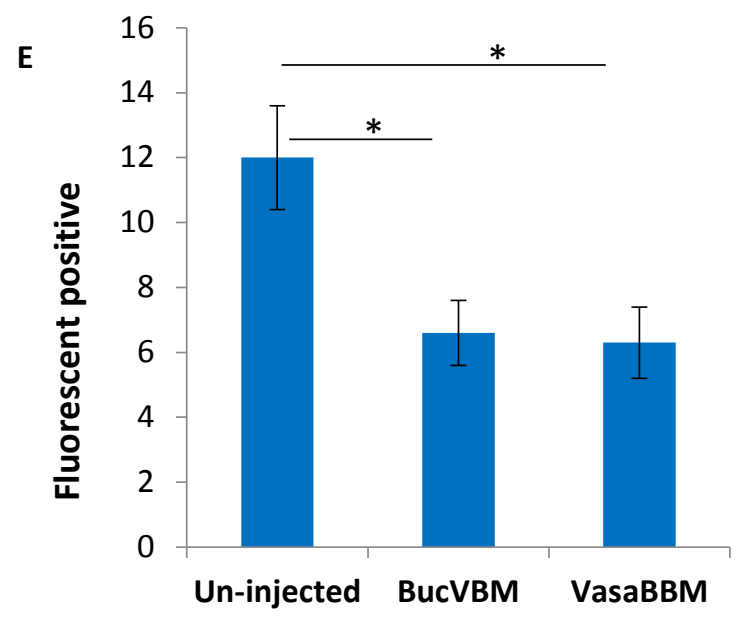

Figure 36: Buc-GFP larvae showed less germ cell after injection of Buc-VBM and Vasa-BBM into Buc-GFP embryos.

(A) A cartoon represents the lateral view of 15-18-somite stage zebrafish larvae, animal to the left. Imaging area boxed in red. (B) Un-injected Buc-GFP embryos showing germ cells $(12 \pm 1.6, n=15)$. (C) Injection of Buc-VBM showed reduction

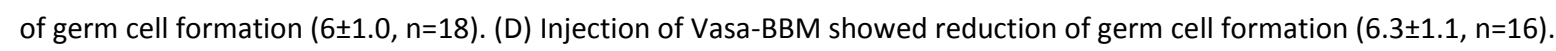
(E) Quantification of number of germ cells. The data presented are averaged from three independent experiments. Therefore, Y-axis represents average germ cell count and X-axis represents injected constructs, Buc-VBM and Vasa-BBM. Error bars represent standard deviation of the mean. Test statistic: Student's t-test. * $\mathrm{P}<0.05$. Scale bar $50 \mu \mathrm{m}$ 
The reduced number of germ cells suggests a dominant-negative effect, which can be explained by an additional molecular interaction, which is required during germ cell specification. In this model, Buc-VBM can interact with endogenous Vasa, but this dimer is not able to bind to a third molecule, which renders the complex inactive. This model predicts that Vasa-BBM would also generate an inactive complex together with endogenous Buc and eventually lead to a reduced number of germ cells. To verify this hypothesis, I overexpressed Vasa-BBM, which should bind to endogenous Buc and form inactive complexes. Indeed, Vasa-BBM overexpression in Buc-eGFP transgenic embryos also showed a reduction of germ cells supporting the hypothesis that Buc-VBM acts as a dominantnegative molecule (Figure 36D). Taken together, these data show that both binding motifs lack another necessary interaction essential for germ cell specification.

\subsection{RNA activates zebrafish Vasa helicase activity}

Recently, it was shown that Oskar binds to nanos RNA (Jeske et al., 2015) in vivo. We previously showed that full length Buc but not a Buc deletion mutant with amino acids 1-362 interacts with nanos3 - 3' UTR (Krishnakumar et al. 2018) in vivo. These data suggest that the C-terminal region of Buc has a potential RNA interaction motif. Moreover, binding of an RNA molecule is necessary to complete the closed conformation of ATPase active site in Vasa to increase the ATPase activity (Jankowsky, 2011). Therefore, I analyzed the ATPase activity of Vasa in the presence of RNA.

After incubation of $50 \mu \mathrm{M}$ ssRNA (polyA $(8)$ ) with Vasa, I observed higher ATPase activity compared to the control without RNA (Figure 37, red vs. green line). As Buc-VBM stimulates the Vasa ATPase activity, I examined ATPase activity in the presence of Buc-VBM together with ssRNA. Fascinatingly, ATPase activity was even stronger in the presence of Buc-VBM and ssRNA compared to the Vasa helicase core with ssRNA (Figure 37, black vs. red line). This result suggests that RNA enhances the activity of the Buc-Vasa complex. Moreover, the interaction with RNA provides an explanation for the reduction of germ cells upon 
overexpression of Buc-VBM and Vasa-BBM, because these peptides might not interact with RNA in vivo.

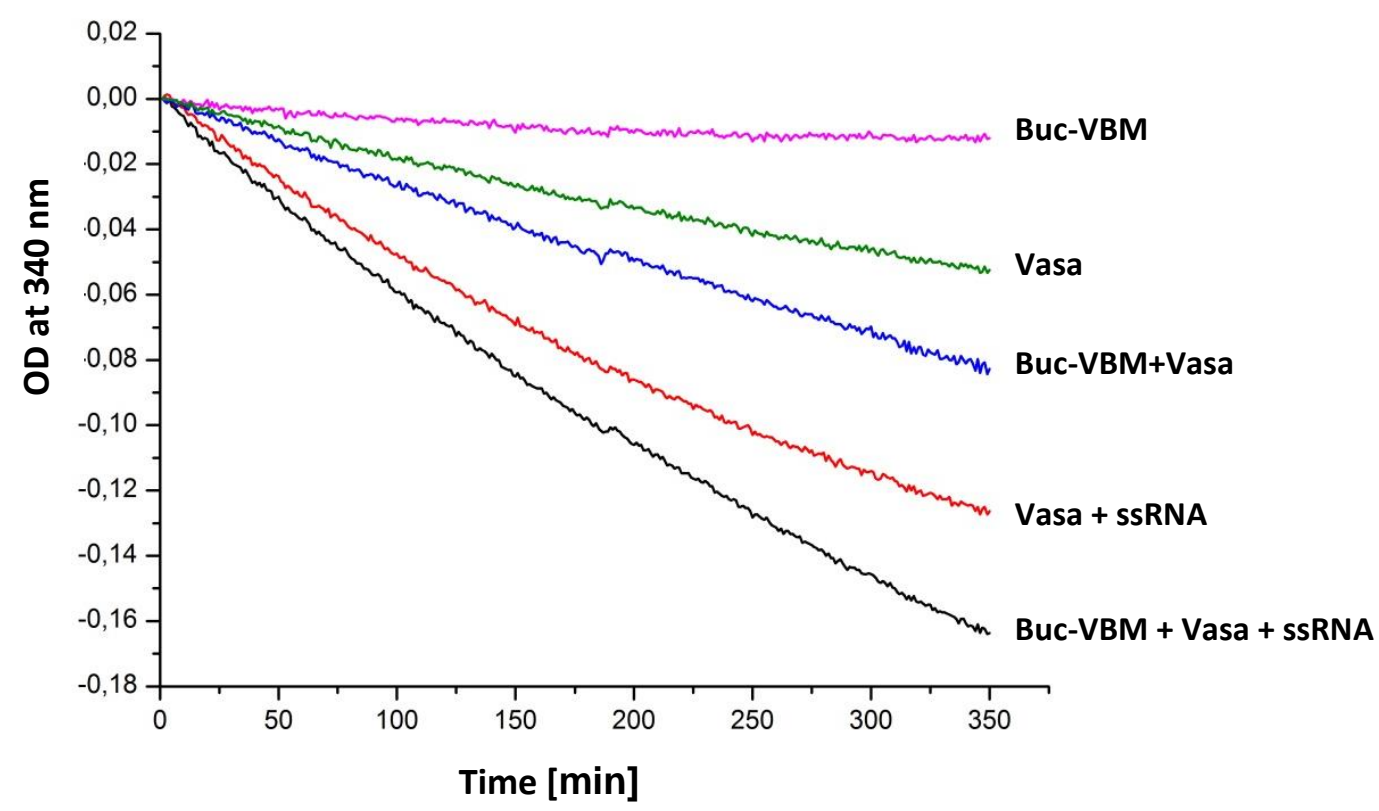

Figure 37: RNA enhances ATPase activity of Vasa.

Buc-VBM activates Vasa helicase activity. Y-axis denotes the reduction of NADH absorption, which is directly proportional to the rate of ATP hydrolysis. The absorbance was measured at $340 \mathrm{~nm}$. X-axis denotes the incubation time in minutes. The data presented are averaged from three independent experiments. Incubation of $100 \mu \mathrm{M}$ Buc-VBM with 2.5 mM ATP showed mild ATPase activity (pink line). Incubation of $5 \mu \mathrm{M}$ Vasa with $2.5 \mathrm{mM}$ ATP demonstrated relatively higher ATPase activity (green line). Incubation of $5 \mu \mathrm{M}$ Vasa with $50 \mu \mathrm{M}$ ssRNA showed enhanced ATPase activity (red line) compared to $5 \mu \mathrm{M}$ Vasa with $100 \mu \mathrm{M}$ Buc-VBM (blue line). Vasa ATPase activity was further enhanced in the presence of ssRNA and Buc-VBM (black line).

\subsection{Amino acid D379 in Buc is required for interaction with Vasa}

My results in Figure 24 showed that Buc $\triangle V B M$ and Vasa $\triangle B B M$ do not interact in vivo with wild-type Vasa and Buc, respectively. Nevertheless, these deletion constructs might not fold into their functional structure without these interaction motifs. Thus, point mutations in the interface of the Buc and Vasa binding site would allow a more meaningful conclusion whether Buc and Vasa interaction is required for germ cell specification. However, there is no structural information available for the Buc-VBM and Vasa interaction. As the Oskar eLOTUS domain interacts with Drosophila Vasa RecA-like C-terminal domain, I aligned the peptide sequence of Buc-VBM and Oskar eLOTUS domain to check whether there are any 
conserved residues between these two peptides. The alignment revealed three amino acids of Buc-VBM, Aspartate (D379), Glutamate (E386), and Serine (S389), which are conserved between two sequences (Figure 38A).

When I compared the position of these three residues with the predicted secondary structures for Buc-VBM, I discovered that D379 is part of the loop region connecting the two predicted $\alpha$-helices, while E386 and S389 are part of the second $\alpha$-helix (Figure 38B).

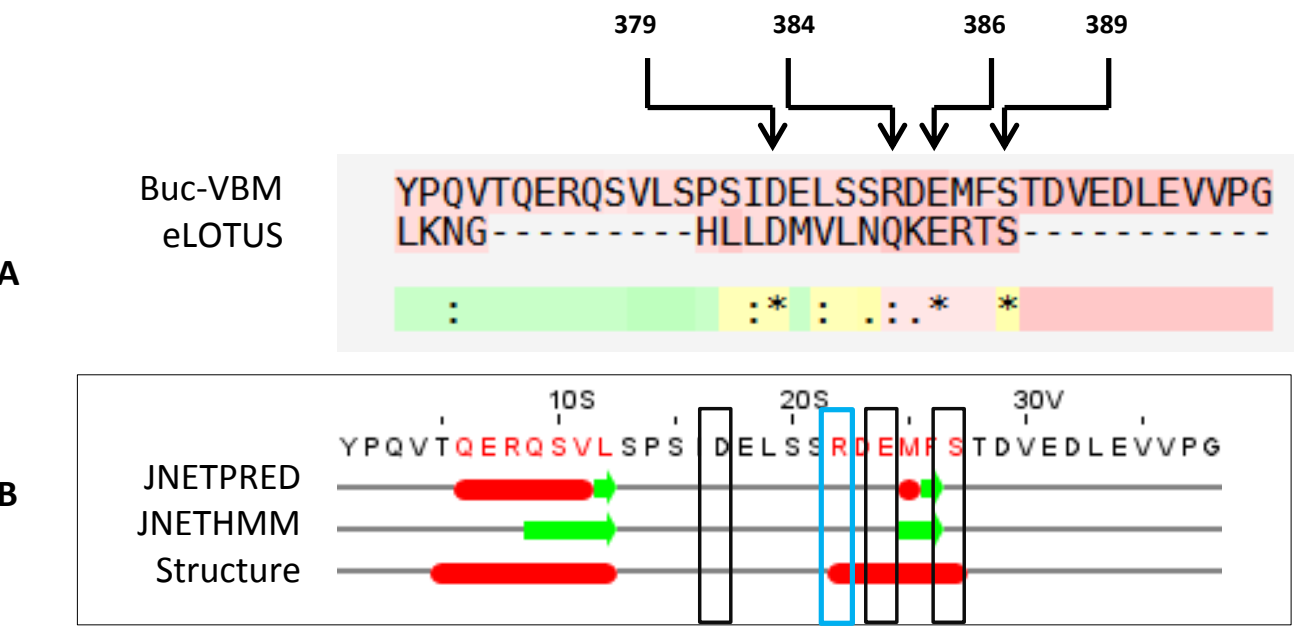

Figure 38: Alignment of Buc-VBM with Oskar eLOTUS domain.

A) Alignment of Buc-VBM and eLOTUS domain revealed that D379, E384 and S386 are conserved between two sequences (black stars and positions of the amino acids are labeled on top of the sequence). In the alignment, an * (asterisk) indicates positions with a fully conserved residue. A ' $:$ ' (colon) denotes conservation based on strongly similar properties - scoring > 0.5 in the PAM 250 matrix. A '? (dot) denotes conservation based on weakly similar properties - scoring $=<0.5$ in the PAM 250 matrix. (B) Representation of the three amino acids D379, E386 and S389 in JPred secondary structure prediction (black boxes) and a non-conserved amino acid R384 (light blue box) that I used as a control during Buc-VBM mutation experiments.

To study the role of these three amino acids for the interaction with Vasa in BiFC assay, I attempted to create point mutations for D379L (Aspartate to Leucine), E386L (Glutamate to Leucine) and S389A (Serine to Alanine) in in full-length Buc. Unfortunately, the site directed mutagenesis failed in many attempts, so I generated these changes in Buc-VBM. As a control, I mutated a non-conserved amino R384G (Arginine to Glycine). Then I fused the Venus-VC fragment to the C-terminus of each mutant construct to examine their activitiy in 
the BiFC assay. After co-injection of D379L, E386L and S389A together with wild-type Vasa, I observed that these mutants Buc showed a reduced fluorescence in the BiFC assay compared to wild-type Buc or Buc R384G (Figure 39).

Among the three mutants, BucD379L had the weakest activity compared to BucE386L and BucS389A (Figure 39). These results suggest that D379 may be a key residue in Buc-VBM for the interaction with Vasa. It seems that the predicted loop region of the Buc-VBM contributes in forming the Buc-Vasa binding interface.

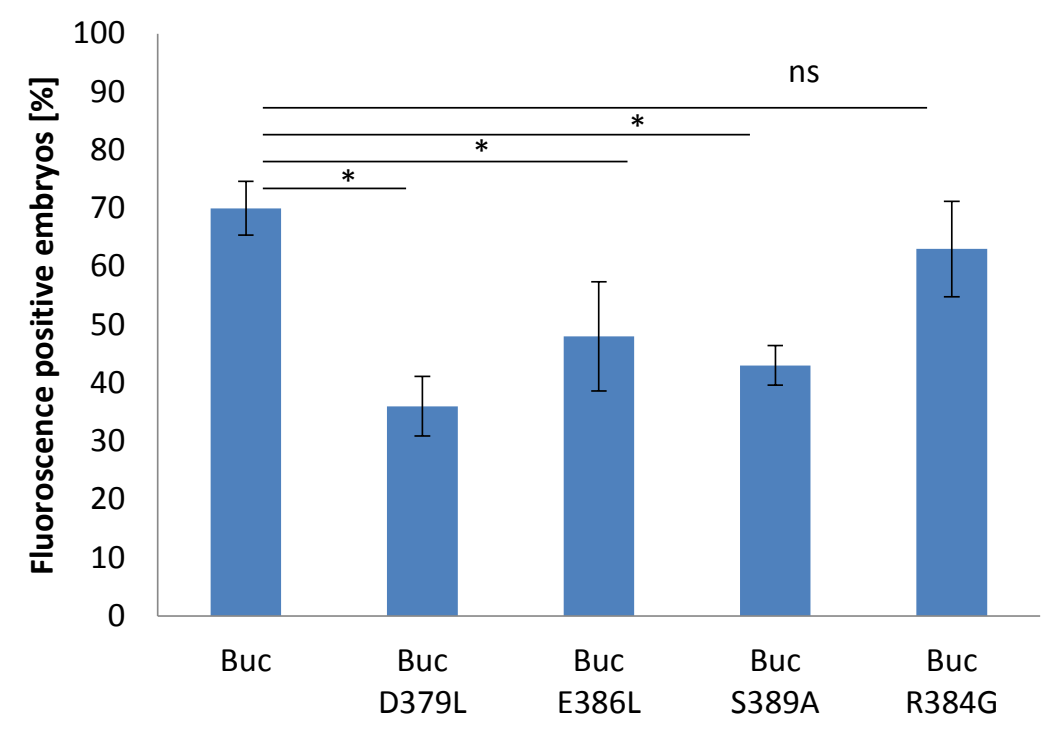

Figure 39: Buc D379L mutation reduces the interaction with Vasa.

The data presented are averaged fluorescent positive embryos from three independent experiments. Therefore, $Y$-axis represents the average fluorescent positive embryos. X-axis represents the injected mutant Buc constructs. Test statistics: Student's t-test, $* P<0.05$. Buc mutants including Buc D379L (36 $\pm 5.1, n=86), E 386 L(48 \pm 9.4, n=77)$, and S389A $(43 \pm 3.4, n=$ $50)$, showed reduction of fluorescent positive embryos compared to wild-type Buc $(70 \pm 4.6, n=69)$ and $R 382 \mathrm{G}(63 \pm 8.2, n=$ 63). Test statistics: Student's t-test, * $\mathrm{P}<0.05$.

\subsection{Homology model predicts three residues in the Vasa-BBM potentially forming an interface during interaction with Buc}

I used the predicted Vasa model as a template to investigate key amino acid residues important to interact with Buc. The predicted Vasa model reveals that the Vasa-BBM consists of one $\alpha$-helix and a ß-sheet with a flexible loop between them (Figure 40). Surface 
representation revealed that three amino acids, S607, S608, 1609, of the flexible loop are exposed on the protein surface (Figure 40B). This suggests that these amino acids have a great probability to interact with Buc-VBM.
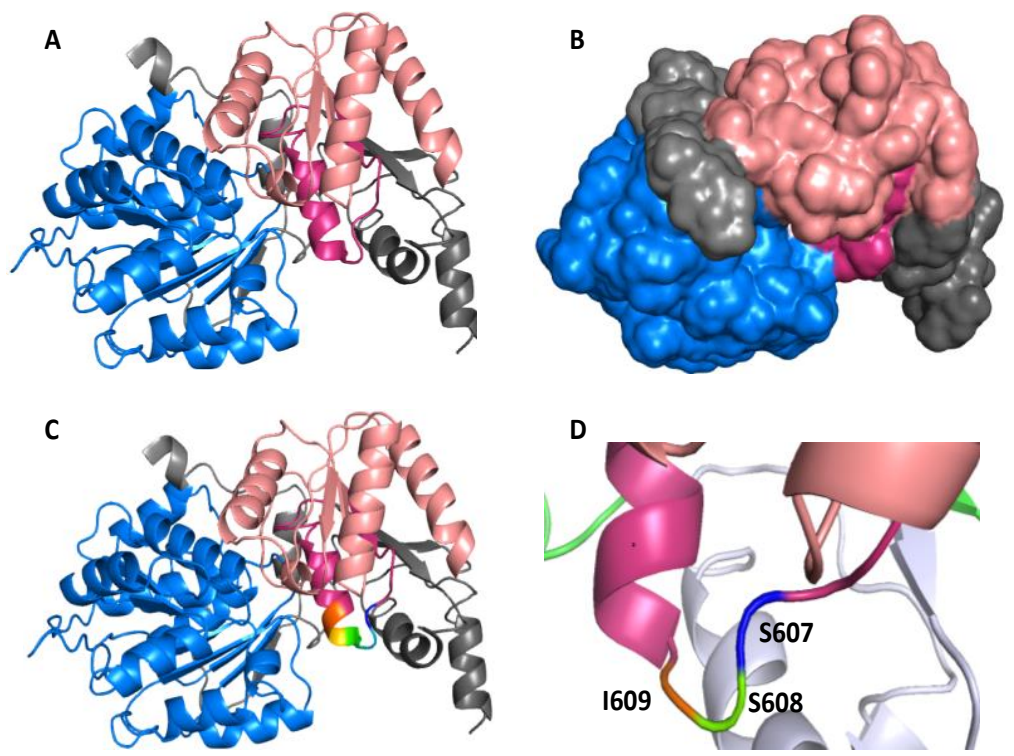

Figure 40: Secondary Structure of Vasa-BBM with exposed amino acids.

(A) Homology model predicted for zebrafish Vasa with RecA like N-terminal domain (blue), RecA like C-terminal domain (light rose) and flanking amino acids (grey). The Vasa-BBM is shown in dark pink. (B) Surface representation of predicted Vasa model showing the exposed region of Vasa-BBM (dark pink). (C) Model highlighting the exposed amino acids in rainbow colors magnified in D. (D) Magnification of exposed amino acids, S607 (blue), S608 (green) and 1609 (orange).

In silico mutagenesis analysis using the PyMol tool (The PyMOL Molecular Graphics System, Version 2.2.3 Schrödinger, LLC, Sengoku et al., 2006) identified that mutating S607A (Serine to Alanine), S608A (Serine to Alanine), and I609Q (Isoleucine to Glutamine) greatly disturbs the structural stability of the flexible loop. This makes these residues primary candidates to address their function to maintain the Vasa-Buc binding-interface. After generation of these mutations in full-length Vasa, I injected mRNA of these variants to perform a BiFC-assay with Buc. While VasaS607A and VasaS608 showed a strongly reduced binding with Buc, Vasal609Q mutant completely lost its interaction with Buc (Figure 41). 


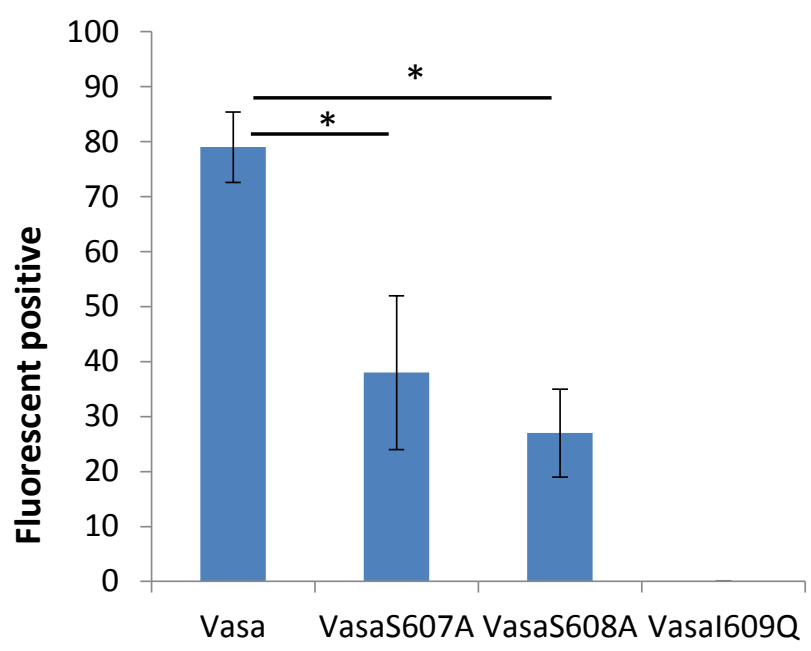

Figure 41: Vasal609Q is critical for interaction with Buc.

The data presented are averaged fluorescent positive embryos from three independent experiments. Therefore, $Y$-axis represents the average fluorescent positive embryos. X-axis represents the injected mutant Vasa constructs. Test statistics: Student's t-test, * $P<0.05$. Compared to wild-type Vasa $(79 \pm 6.4, n=64)$, Vasa S607A $(38 \pm 14.0, n=55)$ and Vasa S608A $(27 \pm 8.0 \mathrm{n}=33)$ showed a reduced number of fluorescence. Fascinatingly, Vasa $16090(0 \pm 0, n=61)$ did not show fluorescent embryos at $3 \mathrm{hpf}$.

To analyze whether the Vasal609Q decreases the stability, I generated a fusion of this Vasa variants with GFP. Overexpression of the same amount of RNA (200 ng/ $\mu$ l) of these Vasa variants shows similar expression levels in vivo as wild-type Vasa-GFP (Figure 42C and D). These data are consistent with the conclusion that Vasa 1609 is not required for the stability of Vasa, but is a critical amino acid for the binding to Buc.

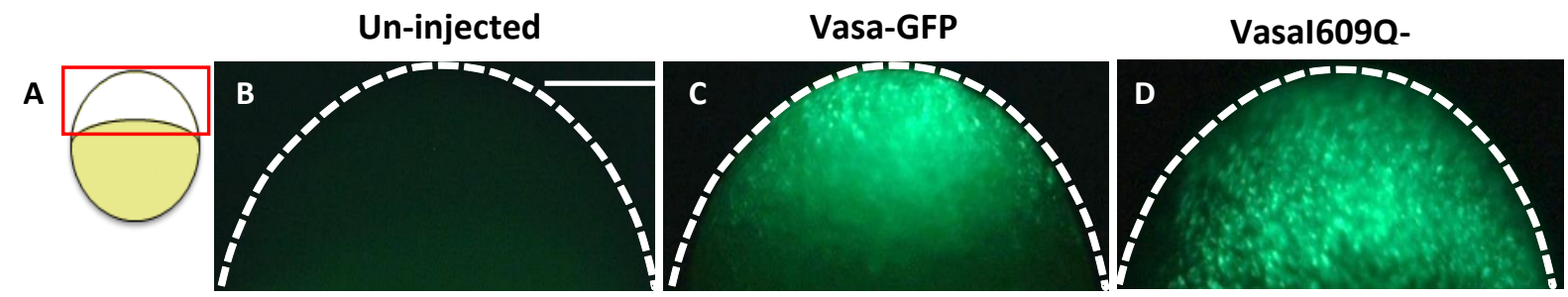

Figure 42: The I609Q mutation does not reduce stability of Vasa in vivo.

(A) A cartoon illustrates the lateral view of embryos at $3 \mathrm{hpf}$, animal to the top. Confocal imaging area boxed in red. This region is outlined with a white dashed line in panel (B-D). (B) Vasal609 showed a similar fluorescent signal in the blastodisc as Vasa-GFP when compared to an un-injected control. Scale bar $200 \mu \mathrm{m}$. 


\subsection{Vasa mutant for ATPase activity induces ectopic germ cells}

I discovered that Vasa directly bind to Buc and that this interaction stimulates the ATPase activity of Vasa in vivo. It has previously been shown that the T546A mutant of Drosophila Vasa abolishes the ATPase activity (Sengoku et al., 2006). However, this variant was only tested in vitro. Moreover, in the year 2006 only RNA was known to stimulate the ATPase activity of Vasa, but it was not discovered yet, that Oskar is required to induce full ATPase activity (Jeske et al., 2017; Sengoku et al., 2006). Further, mutants of Drosophila Vasa of the residues F504E and F508E do not bind to eLOTUS domain of Oskar losing the ability to be activated (Jeske et al., 2017). Taken together, none of these publications addressed whether the ATPase activity is required for germ cell specification in vivo.

To investigate whether the ATPase activity of Vasa is required for germ cell specification, I generated a similar T546A variant of zebrafish Vasa, whose ATPase activity is abolished. Sequence alignment of zebrafish Vasa and Drosophila Vasa indicated that Drosophila Vasa Threonine 546 (T546) is conserved in zebrafish Vasa as T585 (Figure 43A). I mutated T585A (Threonine to Alanine) and injected into a corner somatic cell in 16-cell stage of embryo. Interestingly, I observed that zebrafish mutant Vasa T585A induced ectopic germ cells comparable to wild-type Vasa (Figure 43B and C), suggesting that germ cell induction by Vasa is independent of its ATPase activity. 


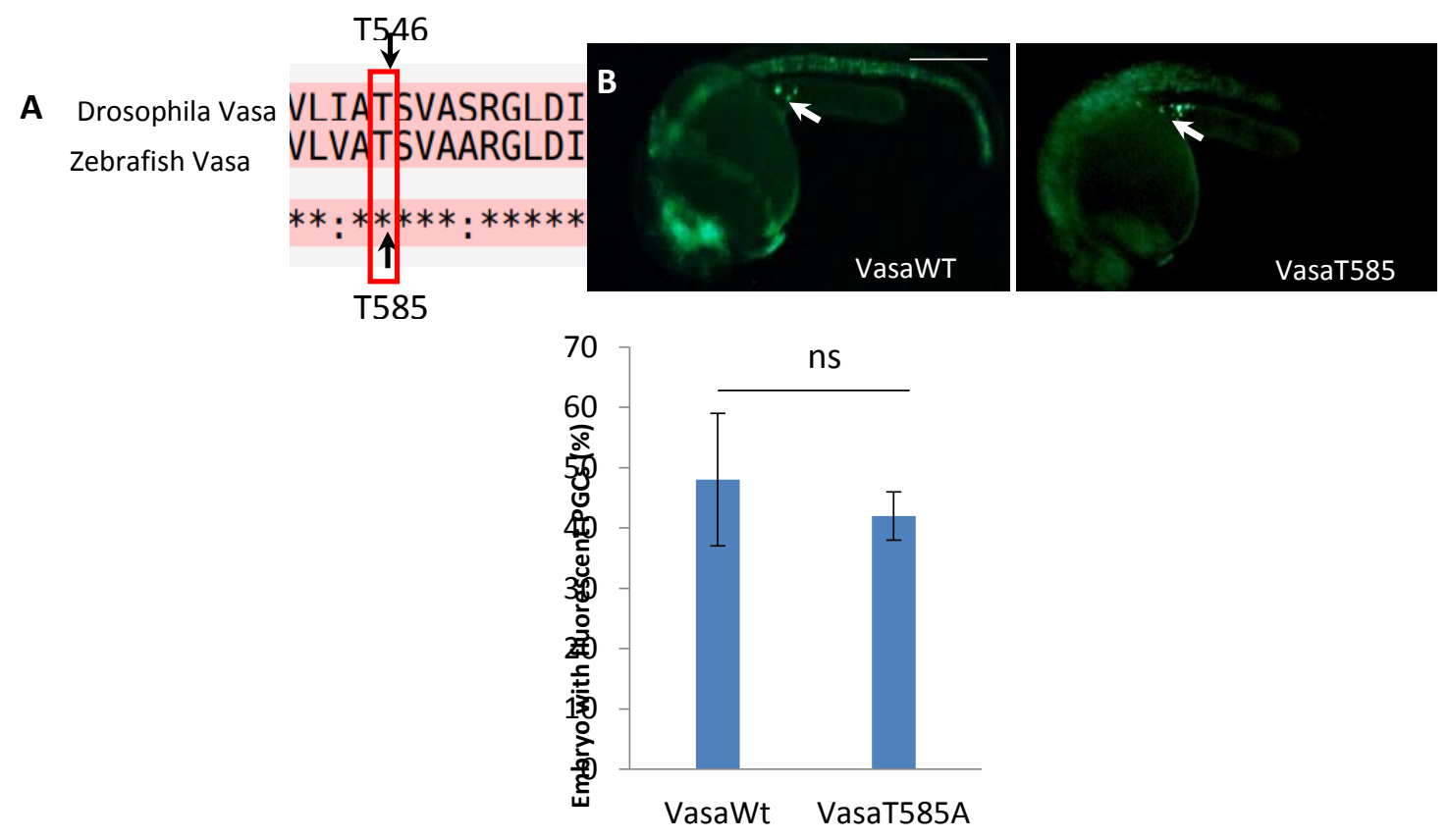

Figure 43: Vasa helicase activity is not required for germ cell induction.

(A) Illustrates partial pair-wise sequence alignment of Drosophila Vasa (amino acid 542-555) and zebrafish Vasa (amino acid 581-594). The conserved Drosophila Vasa T546 and zebrafish Vasa T585 are highlighted by a red rectangle and the corresponding amino acid position is labelled on the sequence. Full sequence alignment is attached in appendix II (B) After 24 hpf, both wild-type Vasa and VasaT585A induced ectopic fluorescent germ cells (white arrow). (C) Quantification of fluorescent germ cells after injection of wild-type Vasa and VasaT585A into a somatic cell at 16-cell stage embryos. The data presented are averaged germ cells from three independent experiments. Therefore, $\mathrm{Y}$-axis represents average fluorescent PGCs and $\mathrm{X}$-axis represents injected constructs. Error bars represent Standard deviation of the mean. Test statistics: Student's t-test. $* \mathrm{P}<0.05$. 


\section{Discussion}

Buc has been recently identified as the first vertebrate protein that organizes in vivo the aggregation of germ plasm. Mutants show that Buc is necessary for germ plasm formation and mRNA injections demonstrate that it is sufficient for the formation of primordial germ cells (Bontems et al., 2009). Zebrafish Buc is specific to vertebrates, but its amino acid sequence encodes a completely novel protein without any known motifs or domains (Bontems et al., 2009). Although Buc is the first vertebrate protein, which has this fascinating activity of inducing germ cells in vivo, its amino acid sequence did not provide any biochemical mechanism, by which it exerts this important function.

Instead of initiating a laborious biochemical approach with Buc, which is also hampered by the insolubility of recombinant protein, we developed an "evolutionary" approach to address the biochemical function of Buc. We recently demonstrated that Buc has an equivalent biological function with the Drosophila germ plasm organizer Oskar i.e. both proteins specify the development of primordial germ cells in zebrafish (Krishnakumar et al., 2018). This is quite remarkable, because Buc and Oskar do not show any sequence homology and an Oskar homolog is not present in the zebrafish genome (Krishnakumar et al., 2018). This publication described for the first time two proteins with equivalent biological function in vivo in the absence of sequence similarity.

To explain this novel phenomenon at the biochemical level, we proposed that Buc and Oskar share a conserved interactome. The evolutionary conserved RNA helicase Vasa has been recognized as a critical component because its interaction is conserved between Buc and Oskar (Jeske et al., 2017, 2015; Krishnakumar et al., 2018). Although, structural and functional details are available for Oskar binding to Vasa, no study investigated the consequence of zebrafish Buc and Vasa interaction during germ cell specification. Thus, the aim of my thesis was to characterize the Buc-Vasa complex and investigate the consequence of their interaction for germ cell specification. 


\subsection{Buc directly binds with Vasa}

The amino acid composition of Buc predicts an intrinsically disordered protein (IDP). Thus, after expression of recombinant Buc protein in Escherichia coli, Buc was found in the insoluble pellet, but not in the soluble fraction. It seems that recombinant Buc protein is highly aggregated in the pellet fraction, because IDPs have a higher tendency to form hydrogen bonds and many electrostatic interactions via charged amino acids (Linding et al., 2004). Hence, recombinant Buc is not available to examine its structural features and biochemical functions. I therefore chose an alternative approach. There, I isolate a small motif, which interacts with Vasa, which then allows me to study the structural features and biochemical functions of the Buc - Vasa interaction.

Previously, it has been shown that Buc interacts with Vasa both in vitro and in vivo (Krishnakumar et al., 2018) using co-immunoprecipitation and the BiFC assay. Coimmunoprecipitation can also be performed using in vitro translated protein in a cell free system or by extraction of cell lysates. However, these samples are complex as they also contain other proteins in addition to the protein to be examined. Thus, coimmunoprecipitation cannot discriminate whether an interaction is direct or not. In the BiFC assay, reconstitution of fluorophore is dependent on their close proximity of two complementary fragments (Kerppola, 2006a, 2008). Therefore, the fluorescent signal detected by the BiFC assay does not reflect whether two proteins interact directly or not. Pull-down assay with recombinant and purified protein is one of the best alternative approaches to show the direct interaction of two proteins. Thus, pulling-down recombinant GST tagged Buc-VBM with Vasa confirmed that Buc directly binds to Vasa in vitro validating the in vivo BiFC results. However, addition of excess amount of Vasa while increasing its concentration did not show strong band for Vasa after Coomassie staining. This suggests that the interaction between Buc and Vasa is weak or transient. We discovered Buc interacts with nanos mRNA (Krishnakumar et al., 2018) and I observed a strong ATPase activity of Vasa in the presence of Buc-VBM and ssRNA. Thus, I speculate that the interaction between Buc and Vasa probably stabilize in the presence of RNA. Nonetheless, the possibility exists that a contamination of the same molecular weight as Vasa is present in the Coomassie stained gel, which might mediate the interaction between Vasa and Buc. Although this possibility is very 
unlikely, one way to test it, would be to cut the Vasa band and sequence the isolated protein by mass-spectrometry. A contaminating protein would then appear by additional sequences.

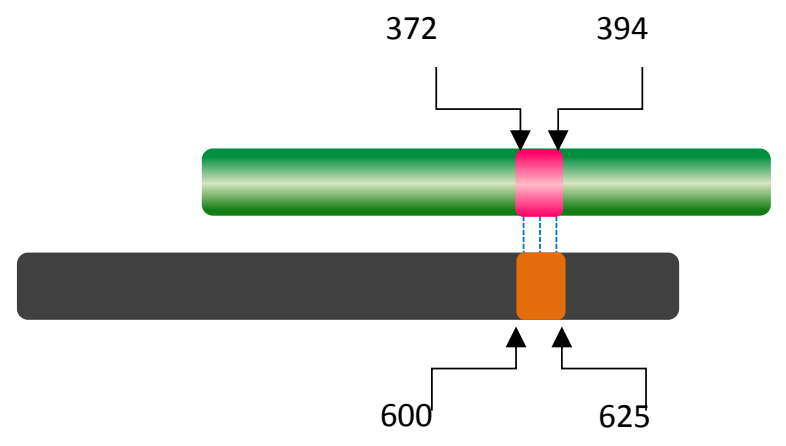

Figure 44: The novel binding motifs in Buc and Vasa.

Full-length of Buc colored in green while Vasa is colored in dark grey. The isolated novel Buc-Vasa binding motif (amino acid 372-394) boxed in pink and Vasa-Buc binding motif (amino acid 600-625) boxed in orange. The direct binding of Buc and Vasa shows in dashed line in blue.

\subsection{The novel interaction motifs in Buc and Vasa}

Based on the BiFC assay, I isolated two novel interaction motifs, Buc-VBM (amino acid 372394) and Vasa-BBM (amino acid 600-625) (Figure 44). The amino acid 372-394 in Buc identifies a highly conserved motif in all Buc orthologs (Figure 45). However, for the first time I discovered that this region is responsible to interact with Vasa. Therefore, this conservation actually suggests that other vertebrates with a conserved Buc protein in their genome also use the interaction with Vasa to specify germ cells.

Using structural predictions and mutagenesis experiments, I discovered that the D379 in Buc-VBM is critical for the interaction with Vasa. As I could not generate a D379L mutation in full-length Buc, it was not possible to study the activity of this point mutation in the germ cell induction assay. A loss of germ cell inducing activity of the D379L mutant would suggest that direct binding of Buc to Vasa is central to induce germ cell development. This model is also consistent with the widespread evolutionary conservation of Vasa as a canonical germline marker (Gustafson \& Wessel, 2010; Juliano et al., 2010). Most importantly, the identification of D379 as a candidate residue in Buc, which is critical for the interaction with Vasa opens new avenues to investigate germ cell development in vertebrates. For instance, it is now theoretically possible to examine the endogenous role of the Buc-Vasa interaction by using CRISPR-Cas9 mediated mutagenesis in zebrafish. Such an experiment would allow 
to confirm in vivo whether Buc is indeed an activator of the Vasa's ATPase activity the context of an entire vertebrate. Similarly, the Vasa-BBM was previously not recognized as an interaction surface for other proteins. Furthermore, Vasa I609Q completely reduced its interaction with Buc. This result suggests that compared to the other direct Buc binding protein such as Rbpms2 (Hermes) (Heim et al., 2014), binding of direct binding of Buc to Vasa is key to induce germ cell development.

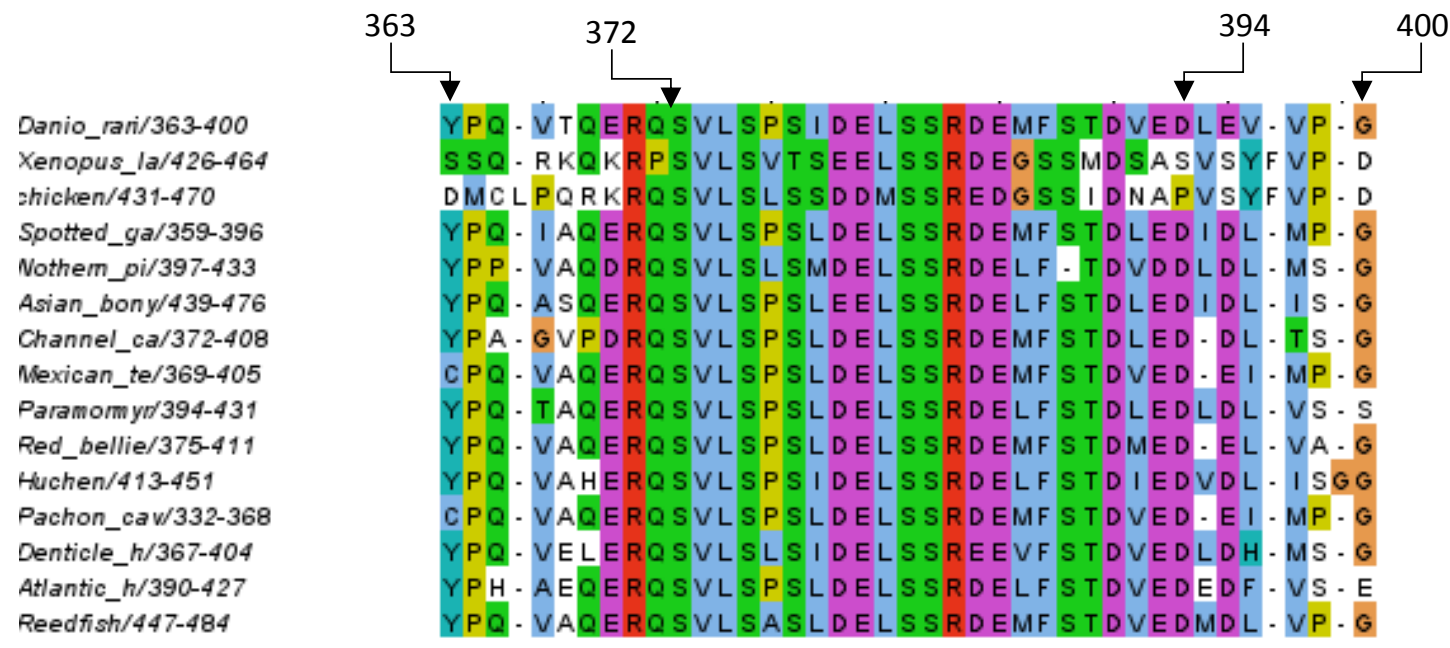

Figure 45: The Buc-VBM is highly conserved among vertebrates.

Alignment of Buc orthologs revealed that the Buc-VBM (amino acid 363-400: labelled on the top of the sequence) is conserved highly conserved among Buc orthologs. Amino acids are colored based on the ClustalX color code. Hydrophobic amino acids are [ Alanine (A), Isoleucine (I), Leucine (L), Methionine (M), Phenylalanine (F), Tryptophan (W), Valine (V)] colored in blue. Positively charge amino acids [(Lysine $(K)$ and Arginine(R)] are colored in red. Negatively charge amino acids [(Aspartic acid (D) and Glutamic acid (E)] are colored in magenta. Polar amino acids (Asparagine (N), Glutamine (Q), Serine $(\mathrm{S})$ and Threonine $(\mathrm{T})$ ] colored in green. Aromatic amino acids (Histidine $(\mathrm{H})$ and Tyrosine $(\mathrm{Y})$ ] colored in cyan. Cystine colored in pink. Glycine colored in orange. Proline colored in yellow

\subsection{Buc and Oskar share structural similarities}

Previously, we showed the functional equivalence between Buc and Drosophila Oskar protein despite no sequence similarity between these two proteins (Krishnakumar et al., 2018). Functional equivalence could be explained by convergent or divergent evolution. In the convergence model, two proteins might have evolved independently to adopt the same function but originate from two independent ancestors. These two genes start their evolution with different genetic mechanisms and selection constraints, but during further 
evolution develop a conserved and common interactome to induce germ cells in two different species. In the divergent model, both proteins might have evolved different sequences from a common ancestor, but maintained a conserved interactome for germ cell specification. The conservation of the common interactome is a response to a similar evolutionary pressure to maintain fertility (Wake et al., 2011; Cusanovich et al., 2014).

Do my results support the convergent or the divergent evolution model of germ plasm organizers? The crystal structure solved for the Drosophila Oskar LOTUS-Vasa complex revealed that the $\alpha 2$ helix of the Drosophila Vasa RecA like C-terminal domain interfaces with $\alpha 2$ helix and $\alpha 5$ helix of the LOTUS domain (Jeske et al., 2017). The $\alpha 2$ helix of the LOTUS domain is one of the trihelical bundle helices of the winged HTH core while the $\alpha 5$ helix is found in the C-terminal region of the LOTUS domain (Figure 29). Remarkably, the crystal structure of the LOTUS domain did not show any secondary structure for the Cterminal region of LOTUS domain suggesting that it is disordered. However, the $\alpha 5$ helix in the LOTUS domain forms as soon as it binds to Vasa suggesting that the secondary structure of the C-terminal extension is induced, when it interacts with Vasa (Figure 30) (Jeske et al., 2017).

To support the stated hypothesis, that Buc and Oskar would share structural identity during their interaction with Vasa, I performed in sillico secondary structure prediction for Buc-VBM as an alternative approach to crystallization. I used CFSSP, PEP2D and Jnet, which are the three predominantly used algorithms in structural biology to predict protein secondary structures. In addition, I performed CD-spectroscopy to measure secondary structures of the Buc-VBM in the presence and absence of the crowding agent Trifluroethanol (TFE). Together, in silico predictions and CD-spectroscopy data revealed that Buc-VBM has a disordered state with a high probability to form $\alpha$-helices in increasing concentrations of crowding agents. Therefore, the Buc-Vasa complex shares some structural identity during their interaction just like the Drosophila Oskar-Vasa complex (Figure 46). 


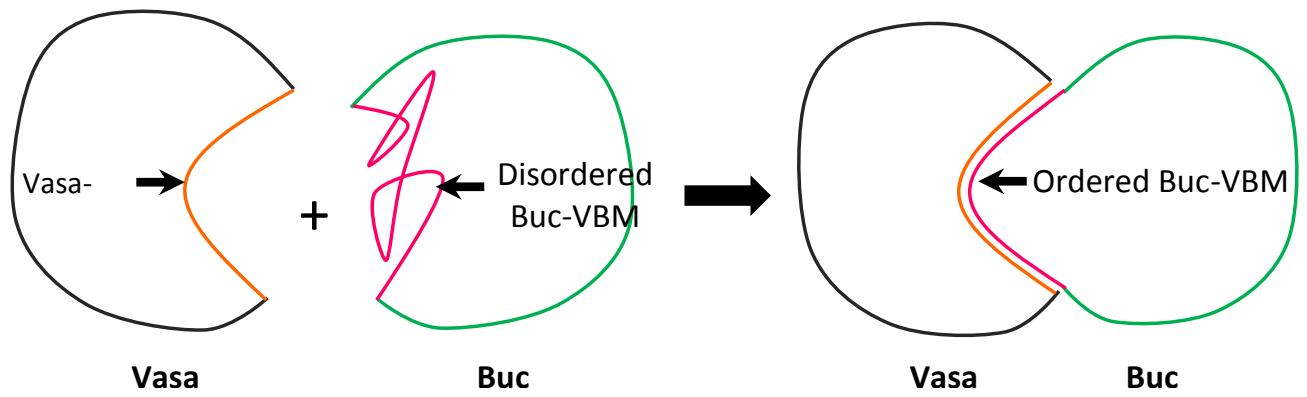

Figure 46: Buc-VBM adopts secondary structure from its disordered state.

. (A) Representation of Vasa and Buc interaction. The Vasa-BBM is colored in orange. Rest of the Vasa sequence colored in dark grey. The disordered Buc-VBM is colored in pink. Rest of the Buc sequence is colored in green (B) The disordered BucVBM adopts ordered structures after interaction with Vasa-BBM.

Indeed, my data showed that amino acids in the predicted helix of Buc (E386 and S389) or in the turn stabilizing the helix (D379) are critical in Buc to interact with Vasa. Remarkably, 1609 in Vasa seems to be a key residue for the interaction with Buc. These findings raise a number of open questions e.g. is there a conserved residue like 1609 in Drosophila Vasa? Which residues in Buc and zebrafish Vasa are indeed involved in stabilizing the contact between the two proteins. However, in depth understating of Buc and Vasa interaction is difficult due to lack of structural data. Therefore, co-crystallization of recombinant Buc-VBM together with Vasa would provide better insight to understand the Buc and Vasa interaction.

During my thesis, I tried to co-crystalize the Buc-Vasa complex. There, I initially performed pre-crystallization tests to find out the appropriate protein concentration of Buc-VBM (amino acid 363-400) and Vasa (amino acid 227-670) for crystallization screening. After setting up the protein crystallization screen, I could not get crystals for the Buc-VBM and Vasa complex. Thereafter, started to optimize the crystallization screen changing various parameters such as buffer condition, protein concentration, addition of additives, $\mathrm{pH}$ and temperature. However, I did not observe any crystal growth after those optimizations. Nevertheless, one way to optimize the co-crystallization is based on an alternative optimization strategy, which is known as the seeding method. For this, I plan to use crystal particles of the DEAH helicase, ctPrp22 (Hamann et al., 2019) due to evolutionary conservation of DEAD and DEAH-box helicase. Therefore, I expect that addition of crystal particles of ctPrp22 crystals into the crystallising solution of Buc-VBM-Vasa complex could probably facilitate crystal growth. 


\subsection{Helicase activity of DEAD box protein is modulated by protein cofactors}

It has been shown that certain RNA binding proteins act as cofactors to stimulate helicase activity of DEAD box helicases (Rogers et al., 2001). For example, eukaryotic translation initiation factor, elF4B stimulates the helicase activities of elF4A and elF4F while elF4H also stimulates the helicase activity of elF4A (Rogers et al., 2001). In addition, yeast cytoplasmic nucleoporin, Gle1 bound to a small molecule, inositol hexakisphosphate (IP6), stimulates the ATPase activity of Dbp5p at the nuclear pore during mRNA export (Bolger \& Wente, 2011).

Moreover, G-patch domain containing proteins have been extensively recognized as cofactors, which stimulate ATPase activity of DEAH RNA helicases (Sloan \& Bohnsack, 2018). For instance, G-patch protein Pfa1p stimulates ATPase activity of the DEAH helicase, Prp43p during yeast ribosome biogenesis (Lebaron et al., 2009). Further, Spp2 acts as a cofactor to accelerate the ATPase activity of Prp2, a DEAH helicase during remodelling the spliceosome complex (Warkocki et al., 2015). These data show that protein cofactors are required to modulate helicase activity of DEAD/H helicases.

\subsection{The Oskar LOTUS domain and Buc-VBM stimulate the helicase activity of Vasa}

Remarkably, it has been shown that the Oskar LOTUS domain stimulates the ATPase activity of Drosophila Vasa upon their interaction albeit Oskar does not belong to any protein family of the known putative helicase regulating cofactors like the mentioned G-patch proteins (Jeske et al., 2017). This study in Drosophila therefore reports for the first-time the active form of Vasa since its discovery (Schupbach \& Wieschaus, 1986). Consistent with this finding, I demonstrated that Buc-VBM indeed activates zebrafish Vasa ATPase activity in vitro. This discovery assigns the first biochemical function to Buc as a novel cofactor, which stimulates the ATPase activity of zebrafish Vasa. 
This result also solves an open question about the role of Vasa in germline development. In some organisms like Drosophila, the Vasa protein is restricted to the germline. By contrast, in other species like zebrafish, Vasa is ubiquitous and then gets restricted to the germline during later embryogenesis. According to my data, the expression of Vasa is not a molecular marker for the prospective germline, but rather its activity. Unfortunately, the product of "active" Vasa is not known. Instead, my data suggest that the expression or localization of the Vasa activator like Buc or Oskar is currently the best molecular marker to predict the future germ cells of the embryo. Unfortunately, Buc and Oskar seem to be restricted to a few species and change their amino acid sequence rapidly, which makes the identification of homologous proteins in other species difficult (Krishnakumar \& Dosch, 2018).

\subsection{Post-translational modification of Vasa and Buc is important for germ line development}

Arginine methylation has been identified as an important post-translational modification (PTM) involved in various biological functions such as RNA processing, protein-protein interactions, DNA repair and gene regulation (Zhu et al., 2019). It has been shown that Vasa undergoes both asymmetric (aDMA) and symmetric dimethylation (sDMA) of arginine by protein methyltransferases (PRMTs) and that this methylation is conserved across phyla (Kirino et al., 2010; Vourekas et al., 2010).

It has been recently reported that in prmt5-/- zebrafish the SDMA of Vasa is disrupted, which resulted in defects in germ cell development during early embryonic development in addition to failure of gonad differentiation in adult zebrafish (Zhu et al., 2019). The defect in germ cell development could be caused by reduced or inhibited interaction between Buc and Vasa as prmt5-/- zebrafish disrupt the sDMA of Vasa. Further, it has been shown that RNA binding protein are the major targets of PRMTs (Pahlich et al., 2006).

As zebrafish Vasa indeed is an RNA binding protein, disruption of sDMA of Vasa would probably have an effect on RNA metabolism, such as mRNA transcription, splicing, transport, translation, and turnover. With my data I showed that Buc stimulates zebrafish Vasa helicase 
activity. Therefore, I believe, that regulation of RNA metabolism involve in germ cell specification pathway is taking place upon Vasa activation by Buc. As a consequence, disruption of SDMA of Vasa probably inhibit its downstream functions related to RNA metabolism, thus, defect in germ cell development.

Moreover, recently it has been shown that the C-terminal region of Buc is a target of sDMAs, which is essential for interaction with Tdrd6a (Roovers et al., 2018). The interaction with Tdrd6a is critical to regulate the solubility, accumulation and mobility of Buc within the germ plasm thereby maintaining liquid-liquid phase separation of germ plasm and eventually PGC formation during early embryogenesis (Roovers et al., 2018).

\subsection{Importance of C-terminal region of Buc during germ cell specification}

Previously I showed that mutant BucP ${ }^{106}$ (amino acid 1-601), which lacks the terminal 38 amino acids and Buc-VBM (amino acid 372-394) do not induce ectopic germ cells in germ cell induction assay. Moreover, we showed that Buc interacts with nanos3 mRNA (Krishnakumar et al., 2018). Physiochemical properties revealed that the terminal 38 amino acids are positively charge as the region is rich in arginine and Lysine. Therefore, we speculated that this region is has a high potential to interact with RNA. Therefore, I hypothesized that in the first step, Buc interacts with RNA. Then Buc binds to Vasa through their Buc-Vasa binding motifs. Thereafter, Buc presents the interacted RNA molecule to Vasa as Vasa involves in RNA processing. Further, the RNA processing will be regulated through triggering Vasa helicase activity stimulated by Buc (Figure 47). My results revealed indeed helicase activity of Vasa is higher in the presence Buc-VBM and RNA (Figure 37) supporting the above hypothesis. Therefore, mapping the RNA binding motif in Buc will provide more details to understand the role of Buc in germ cell specification. 


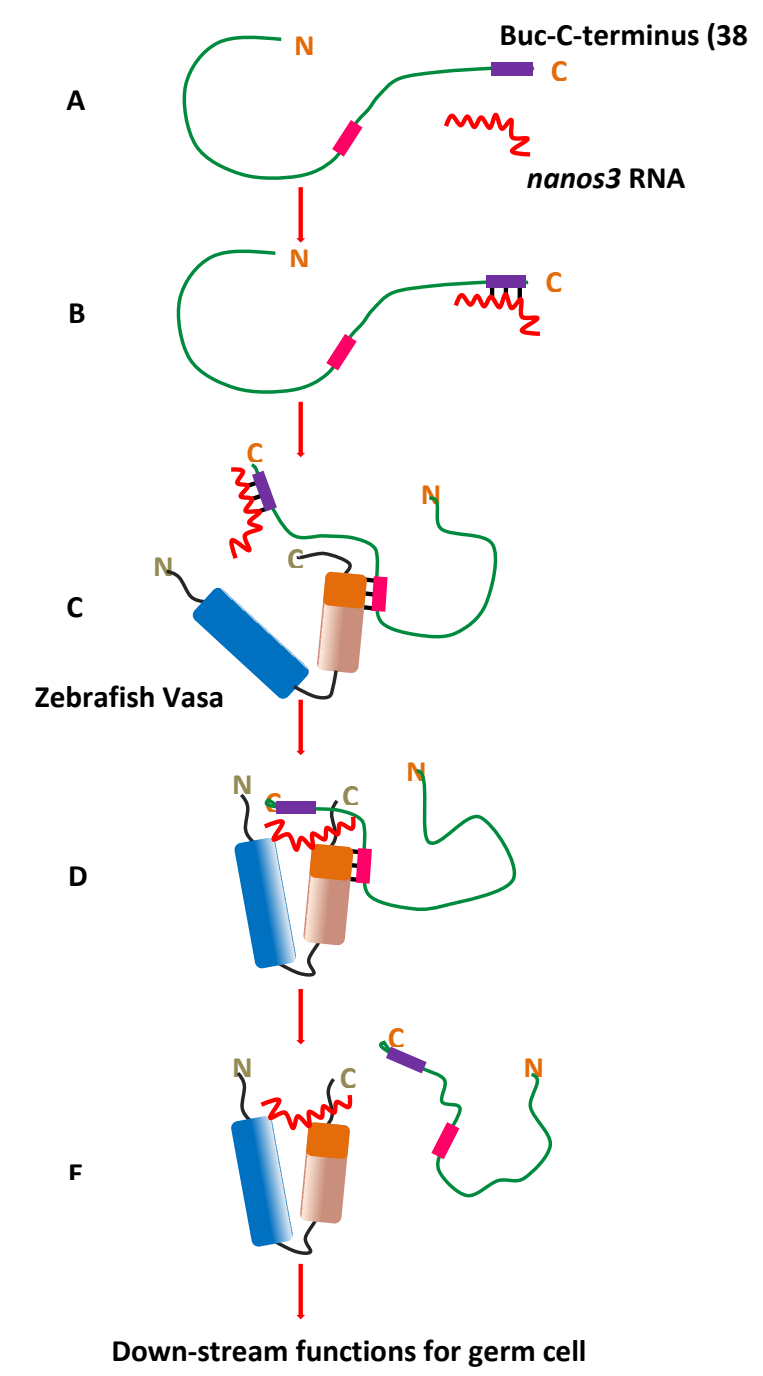

Figure 47: Hypothetical model illustrating potential role of Buc, Vasa, RNA germ cell specification.

(A) Buc protein shown as green line. The Buc-VBM boxed in pink and the terminal 38 amino acids boxed in purple. nanos3 RNA shown in red wavy line. The $\mathrm{N}$-and C-terminus of Buc denoted as ' $\mathrm{N}$ ' and ' $\mathrm{C}$ ' in orange color. (B) nanos3 RNA binds to the C-terminal region of Buc. (C) Vasa protein with its open conformation. The RecA like N-terminal domain of Vasa boxed in blue while RecA like C-terminal domain boxed in rose. The $\mathrm{N}$-and C-terminus of Vasa denoted as ' $\mathrm{N}$ ' and ' $\mathrm{C}$ ' in light brown color. Buc carries nanos3 RNA and bind to Vasa through the Buc-VBM (pink) in Buc and Vasa-BBM (orange) in Vasa. (D) Buc presents the nanos3 RNA to Vasa and Vasa complete its closed conformation. (E) Buc released from the Vasa and Vasa perform its downstream functions to promote germ cell specification.

Considering the interaction of RNA and Tdrd6a with Buc, I speculate that these two interactions probably play a role to establish the specificity of Buc and Vasa interaction during germ cell specification. Because, the mutant BucP ${ }^{106}$ lacks the terminal 38 amino acids. According to the hypothesis, now Buc ${ }^{\mathrm{p} 106}$ cannot interact with RNA or Tdrd6a. As a result, BucP ${ }^{106}$ lacks its specificity for Vasa, even though BucP ${ }^{106}$ has the Vasa binding motif, 
thus resulting in defects in germ cell specification (Figure 48). Further, the defect in PGC formation in $\operatorname{tdrd} 6 a^{-/-}$mutants has been explained by the insufficient accumulation of germ plasm mRNP granules in the absence of Tdrd6a (Roovers et al., 2018). Therefore, Tdrd6a may have other function in addition to regulating Buc-Vasa interaction specificity.

A

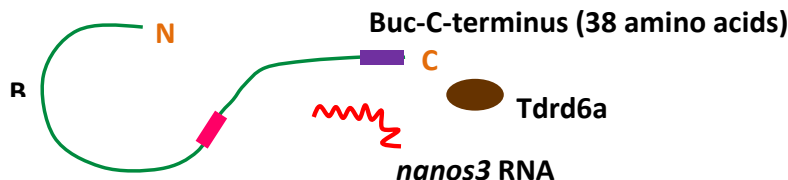

B
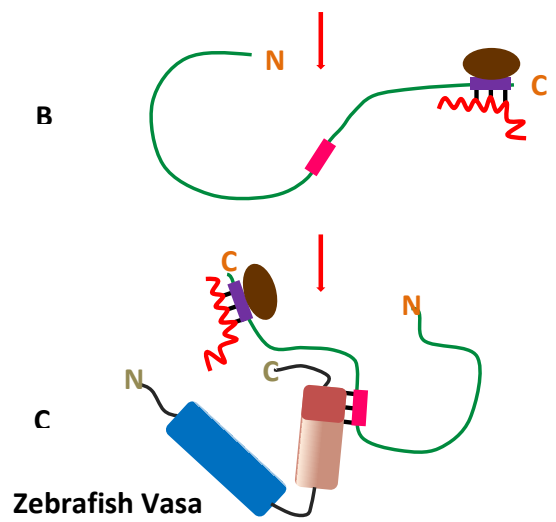

D
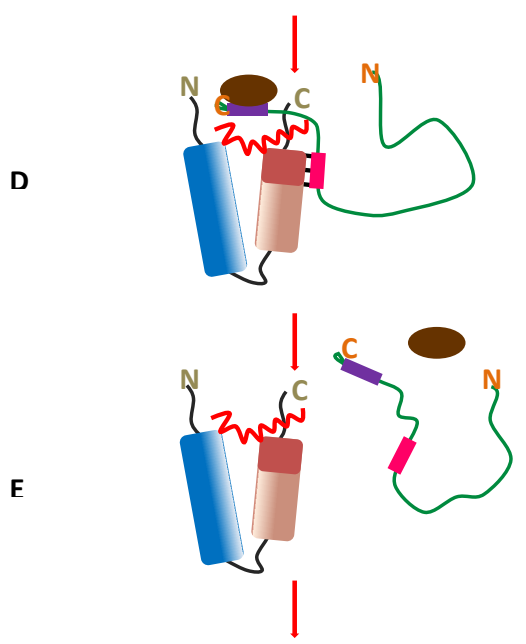

Down-stream functions for germ cell specification

Figure 48: Hypothetical model illustrating potential role of Buc, Vasa, RNA and Tdrd6a during germ cell specification.

(A) Buc protein shown as green line. The Buc-VBM boxed in pink and the terminal 38 amino acids boxed in purple. nanos3 RNA shown in red wavy line and Tdrd6a shown in brown oval. The $\mathrm{N}$-and C-terminus of Buc denoted as ' $\mathrm{N}$ ' and ' $\mathrm{C}$ ' (B) Tdrd6a and nanos3 RNA binds to the C-terminal region of Buc. (C) Vasa protein with its open conformation. The RecA like N-terminal domain of vasa boxed in blue while RecA like C-terminal domain boxed in rose. The $\mathrm{N}$-and C-terminus of Vasa denoted as ' $\mathrm{N}$ ' and ' $\mathrm{C}$ ' in light brown color. Buc carries nanos3 RNA and Tdrd6a and bind to Vasa through the Buc-VBM and Vasa-BBM. (D) Buc presents the nanos3 RNA to Vasa and Vasa complete its closed conformation. (E) Buc and Tdrd6a are released from the Vasa and Vasa perform its downstream functions. 


\subsection{Is the helicase activity of Vasa necessary for germ cell formation?}

I found that Buc-VBM stimulates Vasa helicase activity. Surprisingly, I showed that a Vasa mutant for helicase activity, Vasa T585A induced ectopic germ cells. This finding contradicts the conclusion that germ cell formation requires Vasa's helicase activity. For this conclusion I would first need not to confirm that VasaT585A's has no helicase activity in in vitro assays as performed in Figure 34. Therefore, first, I would need to verify that the Vasa T585A mutation participates in ATP hydrolysis in zebrafish even though it looks conserved with Drosophila T546, which was already shown to abolish Vasa's ATPase activity (Sengoku et al., 2006). Interestingly, the Vasa mutant for helicase activity has never been used in vivo to examine its function in the germline. The reason might be that the helicase activity is not required for germ cell specification.

Alternatively, recently two mutations, $\mathrm{K} 295 \mathrm{~N}$ located in the flanking sequence upstream to the RecA like $\mathrm{N}$-terminal domain and E400Q mutation in conserved DEAD motifs located in the RecA like N-terminal domain in Drosophila Vasa, have been show to interfere with helicase activity (Durdevic \& Ephrussi, 2019; Xiol et al., 2014). I found that these two amino acids are indeed conserved as $\mathrm{K} 326 \mathrm{~N}$ and $\mathrm{E} 435 \mathrm{Q}$ in the RecA like $\mathrm{N}$-terminal domain of zebrafish Vasa. These mutations in Vasa need to be studied first by in vitro ATPase assay followed by germ cell induction assay in zebrafish to investigate importance of helicase activity during germ cell specification.

\subsection{Investigate the hierarchy of germ cell specification pathway}

The investigation of zebrafish germ cell specification pathway after fertilization is technically challenging. For example, it is impossible to investigate protein function after fertilization by genetics, bsince all proteins are produced maternally during oogenesis. One can therefore not mutate an allele in a way, that it loses the maternal contribution of Buc and Vasa during early embryogenesis. Therefore, it needs an alternative approach to remove endogenous protein. It has been reported that endogenous protein can be achieved by using deGradFP (Caussinus et al., 2012) or Trim-away system (Clift et al., 2018). 
deGradFP system contains a Ubquitin binding element and a GFP nanobody. After GFP nanobody binds to GFP moiety of GFP-tagged protein, the protein complex is targeted to ubiquitin-proteasome pathway for protein degradation (Caussinus et al., 2012) Buc-GFP transgenic fish which were generated in the buc mutant background can be used in dGradFP system. To do so, dGradFP mRNA can be injected into one-cell stage Buc-GFP embryos and depletion of Buc can be monitored by checking the GFP signal. If there is no GFP signal after $24 \mathrm{hpf}$ compared to uninjected embryos, it means Buc was degraded and no germ cells have been formed. To confirm no detectable germ cells, embryos can be fixed and stained for germ cell markers, such as Vasa.

Nevertheless, until now no Vasa-GFP transgenic fish line has been generated which rescues the vasa mutant phenotype similar to the Buc-GFP line (cite Riemer). Therefore, the trimaway system provides a great opportunity to degrade maternally provided Vasa during early embryogenesis. The Trim-Away system is based on an E3 ubiquitin ligase called tripartite motif-containing protein 21 (TRIM21). TRIM21 is known to bind antibody-bound pathogens through the Fc domain of bound antibody and then to target them to the ubiquitinproteasome pathway for protein degradation (Clift et al., 2018). To perform the trim-away experiment, anti-Vasa antibody would be injected into one-cell stage embryos. After $24 \mathrm{hpf}$, embryos can be fixed and stained with Buc antibody to check germ cell formation. If germ cells are detected after depletion of Vasa and no germ cells are detected after depleting Buc, it would suggest that Buc is upstream to Vasa and it is necessary for germ cell formation (Figure 48). Nontheless, my results propose for the first time that Buc acts as a novel regulator of Vasa activity thereby integrating this novel protein into known pathways of germline development. 


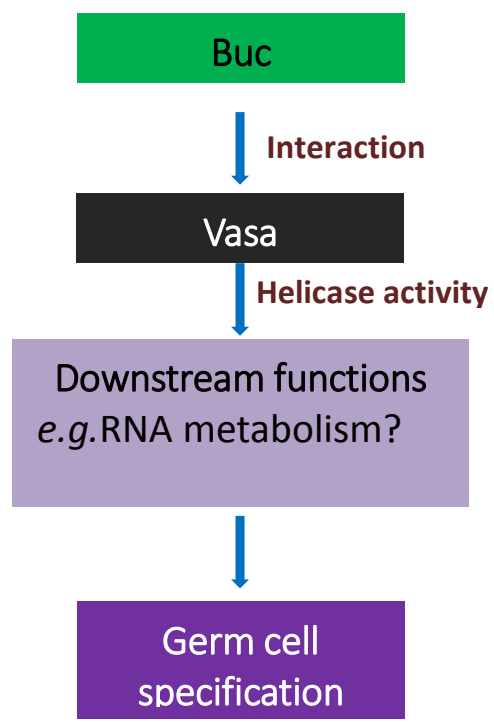

Figure 49: Hypothetical hierarchy of germ cell specification.

According to the data, Buc binds with Vasa. Then Buc stimulates Vasa's helicase activity. The helicase activity 'regulates the downstream functions such as RNA processing during germ cell specification. 


\section{Materials and Methods}

\subsection{Zebrafish handling and maintenance}

Zebrafish (Danio rerio) were used as a model organism. Experiments were performed using $A B^{*}$ TLF zebrafish (wild-type) and Buc-GFP transgenic zebrafish line (Stephan 2015). All the fish were maintained and fed according to the standard protocol (Westerfield, 2000).

\subsection{Manipulation of zebrafish embryos}

\subsubsection{Microinjection}

Pulled $1.0 \mathrm{~mm}$ OD injection needles with a micropipette puller device with following settings, heat $=515$, pull $=228$, vel $=80$, time $=250$. Agarose $(1.5 \%$ in $1 \mathrm{X}$ E3 medium; $(5 \mathrm{mM}$ $\mathrm{NaCl}, 0.17 \mathrm{mM} \mathrm{KCl}, 0.33 \mathrm{mM} \mathrm{CaCl} 2,0.33 \mathrm{mM} \mathrm{MgSO} 4,0.00001 \%$ methylene blue)) plates were prepared placing a mold with wedge-shaped troughs to make grooves in the plates, which facilitates holding embryos during microinjection. Capped sense RNA were diluted in nuclease-free water and $0.025-0.05 \%$ phenol red and $0.1 \mathrm{M} \mathrm{KCl}$. Calibrated the needles in which it injects approximately $0.5 \mathrm{nl}$ of working solution. One-cell stage embryos were injected with $1 \mathrm{nl}$ of diluted RNA with indicated RNA concentration.

\subsubsection{Dechorionation}

Embryos were manually dechorionated using dumont (\#5) watchmaker forceps for imaging purpose. For co-immunoprecipitation experiments, embryos were enzymatically dechorionated using pronase (stock [30 mg/ml] ; Roche, Mannheim) to a final concentration $3 \mathrm{mg} / \mathrm{ml}$ in $1 X$ E3 medium. After 5-10 minutes incubation, floating chorions were decanted and embryos were washed three times with $1 X$ E3 medium. Then, embryos were transferred using fire polished Pasteur pipette. 


\subsubsection{Deyolking}

Approximately 200 dechorionated embryos were transferred into $1 \mathrm{ml}$ of cold $1 / 2$ Ginzburg Fish Ringer deyolking buffer ( $55 \mathrm{mM} \mathrm{NaCl}, 2.7 \mathrm{mM} \mathrm{CaCl} 2,1.8 \mathrm{mM} \mathrm{KCl}, 1.25 \mathrm{mM} \mathrm{NaHCO}$ ). The embryos were triturated using $200 \mu$ pipette tip once and released them back into the buffer slowly. After embryos were shaken for $5 \mathrm{~min}$ at $1100 \mathrm{rpm}$ at $4{ }^{\circ} \mathrm{C}$ to dissolve the yolk. Embryonic cells were pelleted at $1100 \mathrm{rpm}$ for 2 minute and discarded the supernatant. Resuspended the pellet using $1 \mathrm{ml}$ of wash buffer $\left(110 \mathrm{mM} \mathrm{NaCl}, 3.5 \mathrm{mM} \mathrm{KCl}, 2.7 \mathrm{mM} \mathrm{CaCl}_{2}, 10\right.$ $\mathrm{mM}$ Tris/Cl pH8.5) and sample was shaken for $1 \mathrm{~min}$ at $1100 \mathrm{rpm}$. Cells were pelleted at $1100 \mathrm{rpm}$ for 1 minute and discarded the supernatant. Two additional wash steps were performed as described.

\subsubsection{Preparation of embryo lysates}

Deyolked embryonic cell pellets were re-suspended in 2X SDS loading buffer (100 mM Tris (pH 6.8), 20 \% glycerol, 4 \% SDS, 200 mM $\beta$-mercaptoethanol, $0.02 \%$ bromophenol blue). Then samples were incubated at $95{ }^{\circ} \mathrm{C}$ for 5 minutes. Allowed to samples to cool down at room temperature. After that samples were centrifuged at $13000 \mathrm{rpm}$ for 10 minutes to remove contaminants. Supernatant was transferred and used for SDS-polyacrylamide gel electrophoresis.

\subsection{Plasmid vectors and constructs}

\subsubsection{Plasmid vectors for In-Fusion cloning}

pCS2+ multipurpose mammalian expression vector was used to clone BiFC, GFP-fusion and mCherry-fusion protein constructs. 


\subsubsection{Plasmid vectors for Gateway cloning}

Gateway entry clones were generated by using pDONR221 vector while expression clones were generated by using pCSDest or pGCS vectors, which are originated from pCS-vector backbone.

Table 2: Cloned vector and expression construct

\begin{tabular}{|c|c|c|c|}
\hline Plasmid & Vector & Insert & Stratergy \\
\hline \multicolumn{4}{|l|}{ Buc-VBM experiments } \\
\hline pCS2+ BucWT & PCS2+ & buc full-length & (Bontems et al., 2009) \\
\hline pCS2+ BucWT-VC & PCS2+ & buc full-length & $\begin{array}{l}\text { pCS2+VC empty vector used to clone the } \\
\text { construct. In-Fusion cloning primers were } \\
\text { designed selecting BamHI and Xbal sites from the } \\
\text { pCS2+VC vector and the vector was linearized } \\
\text { using the same restriction enzymes. Buc 1-639 } \\
\text { sequence was amplified with using pCS2+ BucWT } \\
\text { as a template. PCR products were recombined } \\
\text { into the linearized vector in a standard In-Fusion } \\
\text { reaction. }\end{array}$ \\
\hline pCS2+ VC-BucWT & PCS2+ & buc full-length & $\begin{array}{l}\text { pCS2+VC empty vector used to clone the } \\
\text { construct. In-Fusion cloning primers were } \\
\text { designed selecting BamHI and Xbal sites from the } \\
\mathrm{pCS} 2+\mathrm{VC} \text { vector and the vector was linearized } \\
\text { using the same restriction enzymes. Buc 1-639 } \\
\text { sequence was amplified with using pCS2+ BucWT } \\
\text { as a template. PCR products were recombined } \\
\text { into the linearized vector in a standard In-Fusion } \\
\text { reaction. }\end{array}$ \\
\hline pCS2+ Buc (1-362aa)-VC & PCS2+ & buc 1-362aa & $\begin{array}{l}\text { pCS2+VC empty vector used to clone the } \\
\text { construct. In-Fusion cloning primers were } \\
\text { designed selecting BamHI and Xbal sites from the } \\
\text { pCS2+VC vector and the vector was linearized } \\
\text { using the same restriction enzymes. Buc 1-362 } \\
\text { sequence was amplified with using pCS2+ BucWT } \\
\text { as a template. PCR products were recombined } \\
\text { into the linearized vector in a standard In-Fusion } \\
\text { reaction. }\end{array}$ \\
\hline
\end{tabular}




\begin{tabular}{|c|c|c|c|}
\hline Plasmid & Vector & Insert & Stratergy \\
\hline pCS2+ Buc (363-639aa)-VC & PCS2+ & buc $363-639$ aa & $\begin{array}{l}\text { pCS2+VC empty vector used to clone thie construct. } \\
\text { In-Fusion cloning primers were designed selecting } \\
\text { BamHI and Xbal sites from the pCS2+VC vector and } \\
\text { the vector was linearized using the same restriction } \\
\text { enzymes. Buc } 363-639 \text { sequence was amplified with } \\
\text { using pCS2+ BucWT as a template. PCR products } \\
\text { were recombined into the linearized vector in a } \\
\text { standard In-Fusion reaction. }\end{array}$ \\
\hline pDONR Buc(363-400aa) & pDONR & buc $363-400$ aa & $\begin{array}{l}\text { Designed gateway attB1FW and attB2RV primers. } \\
\text { Buc } 363-400 \text { sequence was amplified using pCS2+ } \\
\text { BucWT as a template. PCR products were } \\
\text { recombined into pDONR221 vector in a standard } \\
\text { gateway BP cloning reaction. }\end{array}$ \\
\hline pCSDest_Buc(363-400aa)-VC & pCSDest & & $\begin{array}{l}\text { Generated donor vector was recombined into } \\
\text { pCSDest-C-VC vector. }\end{array}$ \\
\hline pDONR Buc(401-639aa) & pDONR & buc $401-639$ aa & $\begin{array}{l}\text { Designed gateway attB1FW and attB2RV primers. } \\
\text { Buc } 400-639 \text { sequence was amplified using pCS2+ } \\
\text { BucWT as a template. PCR products were } \\
\text { recombined into pDONR221 vector in a standard } \\
\text { gateway BP cloning reaction.. }\end{array}$ \\
\hline pCSDest_Buc(401-639)-VC & pCSDest & & $\begin{array}{l}\text { Generated donor vector was recombined into } \\
\text { pCSDest-C-VC vector. }\end{array}$ \\
\hline pDONR Buc(372-394aa) & pDONR & buc $372-394$ aa & $\begin{array}{l}\text { Designed gateway attB1FW and attB2RV primers. } \\
\text { Buc } 372-394 \text { sequence was amplified using pCS2+ } \\
\text { BucWT as a template. PCR products were } \\
\text { recombined into pDONR221 vector in a standard } \\
\text { gateway BP cloning reaction. }\end{array}$ \\
\hline pCSDest_Buc(372-394)-VC & pCSDest & & $\begin{array}{l}\text { Generated donor vector was recombined into } \\
\text { pCSDest-C-VC vector }\end{array}$ \\
\hline
\end{tabular}




\begin{tabular}{|c|c|c|c|}
\hline Plasmid & Vector & Insert & Stratergy \\
\hline pCS2+ BucDetla(372-394aa)-VC & pCS2+ & buc $\Delta 372-394$ aa & $\begin{array}{l}\text { pCS2+VC empty vector used to clone this construct. } \\
\text { In-Fusion cloning primers were designed selecting } \\
\text { BamHI and Xbal sites in addition to the gene specific } \\
\text { overlap PCR primers. The vector was linearized using } \\
\text { the same restriction enzymes. Buc } 1-371 \text { and } 395- \\
639 \text { sequences were amplified using pCS2+ BucWT as } \\
\text { a template. Purified these PCR products were then } \\
\text { mixed in a standard overlap extension PCR reaction } \\
\text { to generate final construct. The final PCR product } \\
\text { was recombined into the linearized vector in a } \\
\text { standard In-Fusion reaction }\end{array}$ \\
\hline $\begin{array}{l}\text { pCS2+ BucDetla(372-394aa)- } \\
\text { GFP }\end{array}$ & pCS2+ & buc $\Delta 372-394$ aa & $\begin{array}{l}\text { pCS2+GFP (stop)vector used to clone this construct. } \\
\text { In-Fusion cloning primers were designed selecting } \\
\text { BamHI and Xbal sites from the pCS2+GFP vector and } \\
\text { the vector was linearized using the same restriction } \\
\text { enzymes. Buc } \triangle 372-394 \text { sequence was amplified } \\
\text { using pCS2+ BucDetla ( } 372-394 a a)-V C \text { as a template. } \\
\text { PCR products was recombined into the linearized } \\
\text { vector in a standard In-Fusion reaction. }\end{array}$ \\
\hline \multicolumn{4}{|c|}{ Buc mutant } \\
\hline pCSDest BucD379L-VC & pCSDest & buc 363-400 aa & $\begin{array}{l}\text { Designed primers with } 15-20 \text { bp of complementary } \\
\text { sequence on either side of the desired mutation. } \\
\text { Complete pCSDest Buc(363-400)-VC vector was } \\
\text { amplified in standard site directed mutagenesis PCR } \\
\text { reaction. Template plasmid was removed by Dpnl } \\
\text { restriction enzyme digestion and } 5 \mu \text { of PCR sample } \\
\text { was transformed into XL1-Blue cells and plated. } \\
\text { Screened colonies for positive mutation. }\end{array}$ \\
\hline pCSDest BucE386L-VC & pCSDest & buc $363-400$ aa & $\begin{array}{l}\text { Designed primers with } 15-20 \text { bp of complementary } \\
\text { sequence on either side of the desired mutation. } \\
\text { Complete pCSDest Buc(363-400)-VC vector was } \\
\text { amplified in standard site directed mutagenesis PCR } \\
\text { reaction. Template plasmid was removed by Dpnl } \\
\text { restriction enzyme digestion and } 5 \mu \text { of PCR sample } \\
\text { was transformed into XL1-Blue cells and plated. } \\
\text { Screened colonies for positive mutation. }\end{array}$ \\
\hline
\end{tabular}




\begin{tabular}{|c|c|c|c|}
\hline Plasmid & Vector & Insert & Stratergy \\
\hline pCSDest BucS389A-VC & pCSDest & buc $363-400$ aa & $\begin{array}{l}\text { Designed primers with } 15-20 \text { bp of complementary } \\
\text { sequence on either side of the desired mutation. } \\
\text { Complete pCSDest Buc(363-400)-VC vector was } \\
\text { amplified in standard site directed mutagenesis PCR } \\
\text { reaction. Template plasmid was removed by Dpnl } \\
\text { restriction enzyme digestion and } 5 \mu \text { of PCR sample } \\
\text { was transformed into XL1-Blue cells and plated. } \\
\text { Screened colonies for positive mutation. }\end{array}$ \\
\hline pCSDest BucR384G-VC & pCSDest & buc $363-400$ aa & $\begin{array}{l}\text { Designed primers with } 15-20 \text { bp of complementary } \\
\text { sequence on either side of the desired mutation. } \\
\text { Complete pCSDest Buc(363-400)-VC vector was } \\
\text { amplified in standard site directed mutagenesis PCR } \\
\text { reaction. Template plasmid was removed by Dpnl } \\
\text { restriction enzyme digestion and } 5 \mu \text { of PCR sample } \\
\text { was transformed into XL1-Blue cells and plated. } \\
\text { Screened colonies for positive mutation. }\end{array}$ \\
\hline \multicolumn{4}{|l|}{ Vasa-BBM experiments } \\
\hline pCSDest VasaWT & pCS2+ & Vasa 1-715 aa & (Krishnakumar et al., 2018) \\
\hline pCS2+ Vasa-VN & pCS2+ & Vasa 1-715 aa & $\begin{array}{l}\text { pCS2+VN empty vector used to clone the construct. } \\
\text { In-Fusion cloning primers were designed selecting } \\
\text { BamHI and Xbal sites from the pCS2+VN vector and } \\
\text { the vector was linearized using the same restriction } \\
\text { enzymes. Vasa 1-715 sequence was amplified using } \\
\text { pCSDest VasaWT as a template. PCR products were } \\
\text { recombined into the linearized vector in a standard } \\
\text { In-Fusion reaction. }\end{array}$ \\
\hline pCS2+ VN-Vasa & & Vasa 1-715 aa & $\begin{array}{l}\text { pCS2+VN empty vector used to clone the construct. } \\
\text { In-Fusion cloning primers were designed selecting } \\
\text { BamHI and Xbal sites from the pCS2+VN vector and } \\
\text { the vector was linearized using the same restriction } \\
\text { enzymes. Vasa 1-715 sequence was amplified with } \\
\text { using pCSDest VasaWT as a template. PCR products } \\
\text { were recombined into the linearized vector in a } \\
\text { standard In-Fusion reaction. }\end{array}$ \\
\hline
\end{tabular}




\begin{tabular}{|c|c|c|c|}
\hline Plasmid & Vector & Insert & Stratergy \\
\hline pCS2+ Vasa(1-277aa)-VN & & Vasa 1-277 aa & $\begin{array}{l}\text { pCS2+VN empty vector used to clone the construct. } \\
\text { In-Fusion cloning primers were designed selecting } \\
\text { BamHI and Xbal sites from the pCS2+VN vector and } \\
\text { the vector was linearized using the same restriction } \\
\text { enzymes. Vasa 1-277 sequence was amplified using } \\
\text { pCSDest VasaWT as a template. PCR products was } \\
\text { recombined into the linearized vector in a standard } \\
\text { In-Fusion reaction. }\end{array}$ \\
\hline pCS2+ Vasa(278-715aa)-VN & pCS2+ & Vasa $278-715$ aa & $\begin{array}{l}\text { pCS2+VN empty vector used to clone the construct. } \\
\text { In-Fusion cloning primers were designed selecting } \\
\text { BamHI and Xbal sites from the pCS2+VN vector and } \\
\text { the vector was linearized using the same restriction } \\
\text { enzymes. Vasa } 278-715 \text { sequence was primers using } \\
\text { pCSDest VasaWT as a template. PCR products were } \\
\text { recombined into the linearized vector in a standard } \\
\text { In-Fusion reaction. }\end{array}$ \\
\hline pDONR Vasa(278-495aa) & pDONR & Vasa $278-495$ aa & $\begin{array}{l}\text { Designed gateway attB1 FW and attB2 RV primers. } \\
\text { Vasa } 278-495 \text { sequence was amplified using pCS2+ } \\
\text { pCSDest VasaWT as a template. PCR products were } \\
\text { recombined into pDONR221 vector in a standard } \\
\text { gateway BP cloning reaction. }\end{array}$ \\
\hline pCSDest Vasa(278-495aa)-VN & pCSDest & & $\begin{array}{l}\text { Generated donor vector was recombined into } \\
\text { pCSDest-C-VN vector. }\end{array}$ \\
\hline pDONR Vasa(496-715aa) & pDONR & Vasa 496-715 aa & $\begin{array}{l}\text { Designed gateway attB1 } \mathrm{FW} \text { and attB2 RV primers. } \\
\text { Vasa } 496-715 \text { sequence was amplified using pCS2+ } \\
\text { pCSDest VasaWT as a template. PCR products were } \\
\text { recombined into pDONR221 vector in a standard } \\
\text { gateway BP cloning reaction. }\end{array}$ \\
\hline pCSDest Vasa(496-715aa)-VN & pCSDest & & $\begin{array}{l}\text { Generated donor vector was recombined into } \\
\text { pCSDest-C-VN vector. }\end{array}$ \\
\hline
\end{tabular}




\begin{tabular}{|c|c|c|c|}
\hline Plasmid & Vector & Insert & Stratergy \\
\hline pDONR Vasa(496-623aa) & pDONR & Vasa 496-623 aa & $\begin{array}{l}\text { Designed gateway attB1 FW and attB2 RV primers. } \\
\text { Vasa } 496-623 \text { sequence was amplified using pCS2+ } \\
\text { pCSDest VasaWT as a template. PCR products were } \\
\text { recombined into pDONR221 vector in a standard } \\
\text { gateway BP cloning reaction. }\end{array}$ \\
\hline pCSDest Vasa(496-623aa)-VN & pCSDest & & $\begin{array}{l}\text { Generated donor vector was recombined into } \\
\text { pCSDest-C-VN vector. }\end{array}$ \\
\hline pDONR Vasa(624-715aa) & pDONR & Vasa 624-715 aa & $\begin{array}{l}\text { Designed gateway attB1 FW and attB2 RV primers. } \\
\text { Vasa 624-715 sequence was amplified using pCS2+ } \\
\text { pCSDest VasaWT as a template. PCR products were } \\
\text { recombined into pDONR221 vector in a standard } \\
\text { gateway BP cloning reaction. }\end{array}$ \\
\hline pDONR Vasa(545-715aa) & pDONR & Vasa 545-715 aа & $\begin{array}{l}\text { Designed gateway attB1 FW and attB2 RV primers. } \\
\text { Vasa } 545-715 \text { sequence was amplified using pCS2+ } \\
\text { pCSDest VasaWT as a template. PCR products were } \\
\text { recombined into pDONR221 vector in a standard } \\
\text { gateway BP cloning reaction. }\end{array}$ \\
\hline pCSDest Vasa(545-715aa)-VN & pCSDest & & $\begin{array}{l}\text { Generated donor vector was recombined into } \\
\text { pCSDest-C-VN vector. }\end{array}$ \\
\hline pDONR Vasa(496-665aa) & pDONR & Vasa 496-665 aa & $\begin{array}{l}\text { Designed gateway attB1 FW and attB2 RV primers. } \\
\text { Vasa 496-665 sequence was amplified using pCS2+ } \\
\text { pCSDest VasaWT as a template. PCR products were } \\
\text { recombined into pDONR221 vector in a standard } \\
\text { gateway BP cloning reaction. }\end{array}$ \\
\hline pCSDest Vasa(496-665aa)-VN & pCSDest & & $\begin{array}{l}\text { Generated donor vector was recombined into } \\
\text { pCSDest-C-VN vector. }\end{array}$ \\
\hline pDONR Vasa(600-715aa) & pDONR & Vasa 600-715 aa & $\begin{array}{l}\text { Designed gateway attB1 FW and attB2 RV primers. } \\
\text { Vasa 600-715 sequence was amplified using pCS2+ } \\
\text { pCSDest VasaWT as a template. PCR products were } \\
\text { recombined into pDONR221 vector in a standard } \\
\text { gateway BP cloning reaction. }\end{array}$ \\
\hline pCSDest Vasa(600-715aa)-VN & pCSDest & & $\begin{array}{l}\text { Generated donor vector was recombined into } \\
\text { pCSDest-C-VN vector. }\end{array}$ \\
\hline
\end{tabular}




\begin{tabular}{|c|c|c|c|}
\hline Plasmid & Vector & Insert & Stratergy \\
\hline pDONR Vasa(545-615aa) & pDONR & Vasa 545-615 aa & $\begin{array}{l}\text { Designed gateway attB1 } \mathrm{FW} \text { and attB2 RV primers. } \\
\text { Vasa } 545-615 \text { sequence was amplified using pCS2+ } \\
\text { pCSDest VasaWT as a template. PCR products were } \\
\text { recombined into pDONR221 vector in a standard } \\
\text { gateway BP cloning reaction. }\end{array}$ \\
\hline pCSDest Vasa(545-615aa)-VN & pCSDest & & $\begin{array}{l}\text { Generated donor vector was recombined into } \\
\text { pCSDest-C-VN vector. }\end{array}$ \\
\hline pDONR Vasa(600-655aa) & pDONR & Vasa 600-655 aa & $\begin{array}{l}\text { Designed gateway attB1 FW and attB2 RV primers. } \\
\text { Vasa } 600-655 \text { sequence was amplified using pCS2+ } \\
\text { pCSDest VasaWT as a template. PCR products were } \\
\text { recombined into pDONR221 vector in a standard } \\
\text { gateway BP cloning reaction. }\end{array}$ \\
\hline pCSDest Vasa(600-655aa)-VN & pCSDest & & $\begin{array}{l}\text { Generated donor vector was recombined into } \\
\text { pCSDest-C-VN vector. }\end{array}$ \\
\hline pDONR Vasa(600-645aa) & pDONR & Vasa 600-645 aa & $\begin{array}{l}\text { Designed gateway attB1 FW and attB2 RV primers. } \\
\text { Vasa 600-645 sequence was amplified using pCS2+ } \\
\text { pCSDest VasaWT as a template. PCR products were } \\
\text { recombined into pDONR221 vector in a standard } \\
\text { gateway BP cloning reaction. }\end{array}$ \\
\hline pCSDest Vasa(595-645aa)-VN & pCSDest & & $\begin{array}{l}\text { Generated donor vector was recombined into } \\
\text { pCSDest-C-VN vector. }\end{array}$ \\
\hline pDONR Vasa(600-635aa) & pDONR & Vasa $600-635$ aa & $\begin{array}{l}\text { Designed gateway attB1 FW and attB2 RV primers. } \\
\text { Vasa 600-635 sequence was amplified using pCS2+ } \\
\text { pCSDest VasaWT as a template. PCR products were } \\
\text { recombined into pDONR221 vector in a standard } \\
\text { gateway BP cloning reaction. }\end{array}$ \\
\hline pCSDest Vasa(595-635aa)-VN & pCSDest & & $\begin{array}{l}\text { Generated donor vector was recombined into } \\
\text { pCSDest-C-VN vector. }\end{array}$ \\
\hline pDONR Vasa(600-625aa) & pDONR & Vasa $600-625$ aa & $\begin{array}{l}\text { Designed gateway attB1 FW and attB2 RV primers. } \\
\text { Vasa } 600-625 \text { sequence was amplified using pCS2+ } \\
\text { pCSDest VasaWT as a template. PCR products were } \\
\text { recombined into pDONR221 vector in a standard } \\
\text { gateway BP cloning reaction. }\end{array}$ \\
\hline
\end{tabular}




\begin{tabular}{|c|c|c|c|}
\hline Plasmid & Vector & Insert & Stratergy \\
\hline pCSDest Vasa(600-625aa)-VN & pCSDest & & $\begin{array}{l}\text { Generated donor vector was recombined into } \\
\text { pCSDest-C-VN vector. }\end{array}$ \\
\hline pCS2+ VasaDetla(600-625)-VN & pCS2+ & Vasa $\Delta 600-655$ aa & $\begin{array}{l}\text { pCS2+VC empty vector used to clone this construct. } \\
\text { In-Fusion cloning primers were designed selecting } \\
\text { BamHI and Xbal sites in addition to the gene specific } \\
\text { overlap PCR primers. The vector was linearized using } \\
\text { the same restriction enzymes. Vasa 1-599 and 626- } \\
715 \text { sequences were amplified using pCSDest } \\
\text { VasaWT as a template. Purified these PCR products } \\
\text { were then mixed in a standard overlap extension PCR } \\
\text { reaction to generate final construct. The final PCR } \\
\text { product was recombined into the linearized vector in } \\
\text { a standard In-Fusion reaction }\end{array}$ \\
\hline pCS2+ VasaDetla(600-625)-GFP & pCS2+ & & $\begin{array}{l}\text { pCS2+GFP (stop) vector used to clone this construct. } \\
\text { In-Fusion cloning primers were designed selecting } \\
\text { BamHI and Xbal sites from the pCS2+GFP vector and } \\
\text { the vector was linearized using the same restriction } \\
\text { enzymes. Vasa delta } 600-625 \text { sequence was } \\
\text { amplified with listed primers using pCS2+ VasaDetla } \\
\text { (600-625aa)-VC as a template. PCR products was } \\
\text { recombined into the linearized vector in a standard } \\
\text { In-Fusion reaction. }\end{array}$ \\
\hline \multicolumn{4}{|l|}{ Vasa mutant } \\
\hline pCS2+Vasa S607A-VN & pCS2+ & vasa $1-715$ aa & $\begin{array}{l}\text { Designed primers with } 15-20 \mathrm{bp} \text { of complementary } \\
\text { sequence on either side of the S607A mutation using } \\
\text { pCS2+Vasa-VN vector. Complete vector was } \\
\text { amplified in standard site directed mutagenesis PCR } \\
\text { reaction. Incubated the PCR sample with Dpnl } \\
\text { restriction enzyme to remove template plasmid. } \\
\text { Then } 5 \mu \text { l of PCR sample was transformed into XL1- } \\
\text { Blue cells and plated. }\end{array}$ \\
\hline
\end{tabular}




\begin{tabular}{|c|c|c|c|}
\hline Plasmid & Vector & Insert & Stratergy \\
\hline pCS2+Vasa S608A-VN & pCS2+ & vasa $1-715$ aa & $\begin{array}{l}\text { Designed primers with } 15-20 \mathrm{bp} \text { of complementary } \\
\text { sequence on either side of the S608A mutation using } \\
\text { pCS2+Vasa-VN vector. Complete vector was } \\
\text { amplified in standard site directed mutagenesis PCR } \\
\text { reaction. Incubated the PCR sample with Dpnl } \\
\text { restriction enzyme to remove template plasmid. } \\
\text { Then } 5 \mu \text { l of PCR sample was transformed into XL1- } \\
\text { Blue cells and plated. }\end{array}$ \\
\hline pDONR Vasal609Q & pCS2+ & vasa $1-715$ aa & $\begin{array}{l}\text { Designed primers with } 15-20 \text { bp of complementary } \\
\text { sequence on either side of the } 1609 \text { Q mutation using } \\
\text { pDONR Vasa vector. Complete vector was amplified } \\
\text { in standard site directed mutagenesis PCR reaction. } \\
\text { Incubated the PCR sample with Dpnl restriction } \\
\text { enzyme to remove template plasmid. Then } 5 \mu \text { of } \\
\text { PCR sample was transformed into XL1-Blue cells and } \\
\text { plated. }\end{array}$ \\
\hline pCSDest Vasal609Q-VN & pCSDest & & $\begin{array}{l}\text { Generated donor vector was recombined into } \\
\text { pCSDest-C-VN vector. }\end{array}$ \\
\hline \multicolumn{4}{|c|}{ Recombinant protein expression vectors } \\
\hline pGEX GST-Buc (363-400aa) & pGEX & buc 363-400 aa & $\begin{array}{l}\text { pGEX GST empty vector used to clone this construct. } \\
\text { In-Fusion cloning primers were designed selecting } \\
\text { EcoRI site. The vector was linearized using the same } \\
\text { restriction enzyme. Buc } 363-400 \text { sequences were } \\
\text { amplified using pCS2+ BucWT as a template. The PCR } \\
\text { products were recombined into the linearized vector } \\
\text { in a standard In-Fusion reaction }\end{array}$ \\
\hline pGEX GST-Vasa(227-670aa) & pGEX & vasa $227-670$ aa & $\begin{array}{l}\text { pGEX GST empty vector used to clone this construct. } \\
\text { In-Fusion cloning primers were designed selecting } \\
\text { BamHI site. The vector was linearized using the same } \\
\text { restriction enzyme. Vasa 227-670 sequence was } \\
\text { amplified using pCSDest VasaWT as a template. The } \\
\text { PCR products were recombined into the linearized } \\
\text { vector in a standard In-Fusion reaction }\end{array}$ \\
\hline
\end{tabular}




\begin{tabular}{|c|c|c|c|}
\hline Plasmid & Vector & Insert & Stratergy \\
\hline \multicolumn{4}{|c|}{ Gateway adopted destination vectors } \\
\hline pCSDest C-VC & pCSDest & & $\begin{array}{l}\text { pCDest empty vector used to clone the construct. In- } \\
\text { Fusion cloning primers were designed selecting Xhol } \\
\text { site. The vector was linearized using the same } \\
\text { restriction enzymes. The Venus C-terminus(VC) } \\
\text { sequences were amplified using pCS2+ VC as a } \\
\text { template. The PCR products were recombined into } \\
\text { the linearized vector in a standard In-Fusion reaction }\end{array}$ \\
\hline pCSDest C-VN & pCSDest & & $\begin{array}{l}\text { pCDest empty vector used to clone the construct. In- } \\
\text { Fusion cloning primers were designed selecting Xhol } \\
\text { site. The vector was linearized using the same } \\
\text { restriction enzymes. The Venus N-terminus(VN) } \\
\text { sequences were amplified using pCS2+ VN as a } \\
\text { template. The PCR products were recombined into } \\
\text { the linearized vector in a standard In-Fusion reaction }\end{array}$ \\
\hline pCSDest N-VC & pCSDest & & $\begin{array}{l}\text { pCDest empty vector used to clone this construct. In- } \\
\text { Fusion cloning primers were designed selecting Clal } \\
\text { site. The vector was linearized using the same } \\
\text { restriction enzymes. The Venus C-terminus(VC) } \\
\text { sequences were amplified using pCS2+ VC as a } \\
\text { template. The PCR products were recombined into } \\
\text { the linearized vector in a standard In-Fusion reaction }\end{array}$ \\
\hline pCSDest N-VN & pCSDest & & $\begin{array}{l}\text { pCDest empty vector used to clone this construct. In- } \\
\text { Fusion cloning primers were designed selecting Clal } \\
\text { site. The vector was linearized using the same } \\
\text { restriction enzymes. The Venus N-terminus(VN) } \\
\text { sequences were amplified using pCS2+ VN as a } \\
\text { template. The PCR products were recombined into } \\
\text { the linearized vector in a standard In-Fusion reaction }\end{array}$ \\
\hline
\end{tabular}


Table 3: Primers used for gateway cloning and In-Fusion cloning

\begin{tabular}{|c|c|c|}
\hline Primer Name & Sequence $\left(5^{\prime}-3^{\prime}\right.$ direction & Cloning purpose \\
\hline BiFC-Buc-VC Forward & $\begin{array}{l}\text { ATCTCCCGGGGGATCCATGGAAGGAATAAAT } \\
\text { AACAATTCA }\end{array}$ & pCS2+ plasmid \\
\hline BiFC-Buc-VC Reverse & $\begin{array}{l}\text { TGTCCATGGTGGATCCGTATCTTGAGCCTCT } \\
\text { TTTCTTCA }\end{array}$ & pCS2+ plasmid \\
\hline BiFC-VC-Buc Forward & $\begin{array}{l}\text { CAAGACCGGTTCTAGAATGGAAGGAATAAAT } \\
\text { AАCAATT }\end{array}$ & pCS2+ plasmid \\
\hline BiFC-VC-Buc Reverse & $\begin{array}{l}\text { AGTCAGGCCTTCTAGTTAGTATCTTGAGCCT } \\
\text { СтTTTCT }\end{array}$ & pCS2+ plasmid \\
\hline Buc (1-362) aa FW & $\begin{array}{l}\text { ATCTCCCGGGGGATCCATGGAAGGAATAAAT } \\
\text { AАCAATT }\end{array}$ & pCS2+ plasmid \\
\hline Buc (1-362) aa RV & $\begin{array}{l}\text { TGTCCATGGTGGATCCGTAGCTGTAGGAATA } \\
\text { AGCACT }\end{array}$ & pCS2+ plasmid \\
\hline Buc (363-639) aa FW & $\begin{array}{l}\text { ATCTCCCGGGGGATCCATGTACCCACAAGTG } \\
\text { ACCC }\end{array}$ & pCS2+ plasmid \\
\hline Buc (363-639)aa RV & $\begin{array}{l}\text { TGTCCATGGTGGATCCGTATCTTGAGCCTCT } \\
\text { TTTCTT }\end{array}$ & pCS2+ plasmid \\
\hline pDONR Buc(401-639)FW & $\begin{array}{l}\text { GGGGACAAGTTTGTACAAAAAAGCAGGCTTA } \\
\text { ATGCATGTTTATGTTGGTGGTGGT }\end{array}$ & pCS2+ plasmid \\
\hline pDONR Buc(401-639)RV & $\begin{array}{l}\text { GGGGACCACTTTGTACAAGAAAGCTGGGTTG } \\
\text { TATCTTGAGCСTCTTTTCTTCATAGAACCT }\end{array}$ & pCS2+ plasmid \\
\hline Buc Delta(372-394)aa overlap FW & $\begin{array}{l}\text { CTCGAAGTTGTTCCAGGGCATGTTTATGTTG } \\
\text { GTGGTGGTAGGC }\end{array}$ & pCS2+ plasmid \\
\hline Buc Delta(372-394)aa overlap RV & $\begin{array}{l}\text { ACTCTGGCGCTCTTGGGTCACTTGTGGGTAG } \\
\text { TAGCTG }\end{array}$ & pCS2+ plasmid \\
\hline Buc D379L FW & $\begin{array}{l}\text { GTCTTAAGTCCATCCATACTTGAGCTCTCCT } \\
\text { CCAGAGAT }\end{array}$ & $\begin{array}{l}\text { SDM into pCDsest } \\
\text { plasmid }\end{array}$ \\
\hline Buc D379L RV & $\begin{array}{l}\text { ATCTCTGGAGGAGAGCTCAAGTATGGATGGA } \\
\text { СТTAAGAC }\end{array}$ & $\begin{array}{l}\text { SDM into pCDsest } \\
\text { plasmid }\end{array}$ \\
\hline
\end{tabular}




\begin{tabular}{|c|c|c|}
\hline Primer Name & Sequence $\left(5^{\prime}-3^{\prime}\right.$ direction & Cloning purpose \\
\hline Buc E386L FW & $\begin{array}{l}\text { GAGCTCTCCTCCAGAGATCTAATGTTCTCCACTGAT } \\
\text { GTA }\end{array}$ & $\begin{array}{l}\text { SDM into pCDsest } \\
\text { plasmid }\end{array}$ \\
\hline Buc E386L RV & $\begin{array}{l}\text { TACATCAGTGGAGAACATTAGATCTCTGGAGGAGAG } \\
\text { CTC }\end{array}$ & $\begin{array}{l}\text { SDM into pCDsest } \\
\text { plasmid }\end{array}$ \\
\hline Buc S389A_1 FW & $\begin{array}{l}\text { TCCAGAGATGAAATGTTCGCCACTGATGTAGAGGAT } \\
\text { СTC }\end{array}$ & $\begin{array}{l}\text { SDM into pCDsest } \\
\text { plasmid }\end{array}$ \\
\hline Buc S389A_1 RV & $\begin{array}{l}\text { GAGATCCTCTACATCAGTGGCGAACATTTCATCTCT } \\
\text { GGA }\end{array}$ & $\begin{array}{l}\text { SDM into pCDsest } \\
\text { plasmid }\end{array}$ \\
\hline Buc R384G FW & $\begin{array}{l}\text { CATAGATGAGCTCTCCTCCGGAGATGAAATGTTCTC } \\
\text { CACTG }\end{array}$ & $\begin{array}{l}\text { SDM into } \mathrm{pCDsest} \\
\text { plasmid }\end{array}$ \\
\hline Buc R384G RV & $\begin{array}{l}\text { CAGTGGAGAACATTTCATCTCCGGAGGAGAGCTCAT } \\
\text { CTATG }\end{array}$ & $\begin{array}{l}\text { SDM into pCDsest } \\
\text { plasmid }\end{array}$ \\
\hline BiFC- Vasa-VN FW & ATCTCCCGGGGGATCCATGGATGACTGGGAGGAAG & pCS2+ plasmid \\
\hline BiFC- Vasa-VN RV & TCACCATGGTGGATCCTTCCCATTCCTCATCGTCT & pCS2+ plasmid \\
\hline BiFC-VN-Vasa FW & GGCCACCGGTTCTAGAATGGATGACTGGGAGGAAG & pCS2+ plasmid \\
\hline BiFC-VN-Vasa RV & $\begin{array}{l}\text { AGTCAGGCCTTCTAGTTATTCCCATTCCTCATCGT } \\
\text { C }\end{array}$ & pCS2+ plasmid \\
\hline BiFC Vasa(1-277)aa FW & ATCTCCCGGGGGATCCATGGATGACTGGGAGGA & pCS2+ plasmid \\
\hline BiFC Vasa(1-277)aa RV & TCACCATGGTGGATCCAGTCATAATTGCTTTTGGA & pCS2+ plasmid \\
\hline BiFC Vasa(278-715)aa FW & ATCTCCCGGGGGATCCATGTTTGAGGAAGCAGG & pCS2+ plasmid \\
\hline BiFC Vasa(278-715)aa RV & TCACCATGGTGGATCCTTCCCATTCCTCATCG & pCS2+ plasmid \\
\hline pDONR vasa(278-495)aa FW & $\begin{array}{l}\text { GGGGACAAGTTTGTACAAAAAAGCAGGCTTAATGT } \\
\text { TTGAGGAAGCAGGACTTTGT }\end{array}$ & pDONR plasmid \\
\hline pDONR vasa(278-495)aa RV & $\begin{array}{l}\text { GGGGACCACTTTGTACAAGAAAGCTGGGTTCACAC } \\
\text { CAACAGCAAGGAAAATA }\end{array}$ & pDONR plasmid \\
\hline
\end{tabular}




\begin{tabular}{|c|c|c|}
\hline Primer Name & Sequence $\left(5^{\prime}-3^{\prime}\right.$ direction & Cloning purpose \\
\hline pDONR Vasa(496-715)aa FW & $\begin{array}{l}\text { GGGGACAAGTTTGTACAAAAAAGCAGGCTTAATGG } \\
\text { TGGGTGGAGCATGCAGT }\end{array}$ & pDONR plasmid \\
\hline pDONR Vasa(1-715)aa NS RV & $\begin{array}{l}\text { GGGGACCACTTTGTACAAGAAAGCTGGGTTTTCCC } \\
\text { АтTССTCATСGTCTGCAG }\end{array}$ & pDONR plasmid \\
\hline pDONR Vasa(496-715)aa FW & $\begin{array}{l}\text { GGGGACAAGTTTGTACAAAAAAGCAGGCTTAATGG } \\
\text { TGGGTGGAGCATGCAGT }\end{array}$ & pDONR plasmid \\
\hline pDONR Vasa(496-623)aa RV & $\begin{array}{l}\text { GGGGACCACTTTGTACAAGAAAGCTGGGTTCCCAC } \\
\text { AGCGTCCAGTTCT }\end{array}$ & pDONR plasmid \\
\hline pDONR Vasa (624-715)aa FW & $\begin{array}{l}\text { GGGGACAAGTTTGTACAAAAAAGCAGGCTTAATGG } \\
\text { GGAACACCGGTCG }\end{array}$ & pDONR plasmid \\
\hline pDONR Vasa(545-715)aa FW & $\begin{array}{l}\text { GGGGACAAGTTTGTACAAAAAAGCAGGCTTAATGA } \\
\text { САтTTСтСтGTCAAGAGAAGATCTCAAC }\end{array}$ & pDONR plasmid \\
\hline pDONR Vasa(496-665)aa RV & $\begin{array}{l}\text { GGGGACCACTTTGTACAAGAAAGCTGGGTTGAAGG } \\
\text { САACTTССTCCAGCC }\end{array}$ & pDONR plasmid \\
\hline pDONR Vasa(600-655)aa FW & $\begin{array}{l}\text { GGGGACAAGTTTGTACAAAAAAGCAGGCTTAATGG } \\
\text { TTGTGAATTTTGACATGCCC }\end{array}$ & pDONR plasmid \\
\hline pDONR Vasa(545-715)aa FW & $\begin{array}{l}\text { GGGGACAAGTTTGTACAAAAAAGCAGGCTTAATGA } \\
\text { САTTTCTCTGTCAAGAGAAGATCTCAAC }\end{array}$ & pDONR plasmid \\
\hline pDONR Vasa(545-615)aa RV & $\begin{array}{l}\text { GGGGACCACTTTGTACAAGAAAGCTGGGTTATGGA } \\
\text { САTACTCATCGATGCTGC }\end{array}$ & pDONR plasmid \\
\hline pDONR Vasa(545-655)aa RV & $\begin{array}{l}\text { GGGGACCACTTTGTACAAGAAAGCTGGGTTAACTA } \\
\text { СтTGTTGGGCСССTGAAAG }\end{array}$ & pDONR plasmid \\
\hline pDONR Vasa(600-645)aa RV & $\begin{array}{l}\text { GGGGACCACTTTGTACAAGAAAGCTGGGTTGACCA } \\
\text { GGGAGCGAGCTAATG }\end{array}$ & pDONR plasmid \\
\hline pDONR Vasa(600-635)aa RV & $\begin{array}{l}\text { GGGGACCACTTTGTACAAGAAAGCTGGGTTTGGGT } \\
\text { TAAAAAAGGACACGGCG }\end{array}$ & pDONR plasmid \\
\hline pDONR Vasa(600-625)aa RV & $\begin{array}{l}\text { GGGGACCACTTTGTACAAGAAAGCTGGGTTGTTCC } \\
\text { CACAGCGTCCAGTTCT }\end{array}$ & pDONR plasmid \\
\hline
\end{tabular}




\begin{tabular}{|c|c|c|}
\hline Primer Name & Sequence $\left(5^{\prime}-3^{\prime}\right.$ direction & Cloning purpose \\
\hline Vasa Delata(600-625) Overlap FW & $\begin{array}{l}\text { GAGCAAGTCCAGCATGTTGGTCGCGCCGTGTC } \\
\text { СтTTTTTAAC }\end{array}$ & pCS2+ plasmid \\
\hline Vasa Delata(600-625) Overlap RV & $\begin{array}{l}\text { AAAGGACACGGCGCGACCAACATGCTGGACTT } \\
\text { GCTCAATATCCAG }\end{array}$ & pCS2+ plasmid \\
\hline pCSDesVasa_S607A_FW & $\begin{array}{l}\text { GTGAATTTTGACATGCCCGCCAGCATCGATGA } \\
\text { GTATGTCCATC }\end{array}$ & $\begin{array}{l}\text { SDM into pCSDest } \\
\text { plasmid }\end{array}$ \\
\hline pCSDesVasa_S607A_RV & $\begin{array}{l}\text { GATGGACATACTCATCGATGCTGGCGGGCATG } \\
\text { TCAAAATTCAC }\end{array}$ & $\begin{array}{l}\text { SDM into pCSDest } \\
\text { plasmid }\end{array}$ \\
\hline pCSDesVasa_S608A_FW & $\begin{array}{l}\text { TGAATTTTGACATGCCCAGCGCCATCGATGAG } \\
\text { TATGTCCATCG }\end{array}$ & $\begin{array}{l}\text { SDM into pCSDest } \\
\text { plasmid }\end{array}$ \\
\hline pCSDesVasa_S608A_RV & $\begin{array}{l}\text { CGATGGACATACTCATCGATGGCGCTGGGCAT } \\
\text { GTCAAAATTCA }\end{array}$ & $\begin{array}{l}\text { SDM into pCSDest } \\
\text { plasmid }\end{array}$ \\
\hline pDONR Vasa(1-715)aa FW & $\begin{array}{l}\text { GGGGACAAGTTTGTACAAAAAAGCAGGCTTAA } \\
\text { TGGATGACTGGGAGGAAGATCAG }\end{array}$ & pDONR plasmid \\
\hline pCSDesVasa_1609Q_FW & $\begin{array}{l}\text { GAATTTTGACATGCCCAGCAGCCAGGATGAGT } \\
\text { ATGTCCATCGCATAG }\end{array}$ & $\begin{array}{l}\text { SDM into pCSDest } \\
\text { plasmid }\end{array}$ \\
\hline pCSDesVasa_1609Q_RV & $\begin{array}{l}\text { CTATGCGATGGACATACTCATCCTGGCTGCTG } \\
\text { GGCATGTCAAAATTC }\end{array}$ & $\begin{array}{l}\text { SDM into pCSDest } \\
\text { plasmid }\end{array}$ \\
\hline pGEX_Inf Buc(363-400)aa FW & $\begin{array}{l}\text { GGGATCCCCGGAATTCTACCCACAAGTGACCC } \\
\text { AAGAGC }\end{array}$ & pGEX plasmid \\
\hline pGEX_Inf Buc(363-400)aa RV & $\begin{array}{l}\text { GTCGACCCGGGAATTTTACCCTGGAACAACTT } \\
\text { CGAGATCC }\end{array}$ & pGEX plasmid \\
\hline pGEX Vasa 227-660aa FW & $\begin{array}{l}\text { GGGGCCCCTGGGATCAAATGCAGGACCCAAGG } \\
\text { TTGTTTATG }\end{array}$ & pGEX plasmid \\
\hline pGEX Vasa 227-670aa RV & $\begin{array}{l}\text { GGAATTCCGGGGATCCTATGTTGTCCCATGAG } \\
\text { САСTGAAG }\end{array}$ & pGEX plasmid \\
\hline
\end{tabular}




\subsection{Molecular biology methods}

\subsubsection{Polymerase chain reaction (PCR)}

Target DNA fragments were amplified in a standard PCR reaction (Mullis et al., 1986) as shown in table 3 and 4.

Table 4: Standard PCR reaction

\begin{tabular}{|l|c|l|}
\hline Reagent & Volume & Final concentration \\
\hline $\begin{array}{l}5 \mathrm{X} \text { Phusion HF buffer } \\
\text { (New England BioLabs, Ipswich, USA) }\end{array}$ & $10.0 \mu \mathrm{l}$ & $1 \mathrm{X}$ \\
\hline $\begin{array}{l}\text { Forward primer }(10 \mu \mathrm{M}) \\
\text { (Sigma Aldrich, Hannover) }\end{array}$ & $2.5 \mu \mathrm{l}$ & $0.5 \mu \mathrm{M}$ \\
\hline Reverse primer (10 $\mu \mathrm{M})$ & $2.5 \mu \mathrm{l}$ & $0.5 \mu \mathrm{M}$ \\
(Sigma Aldrich, Hannover) & $2.0 \mu \mathrm{l}$ & $200 \mu \mathrm{M}$ \\
\hline $\begin{array}{l}\text { dNTPs (5mM) } \\
\text { (Thermo Scientific, USA) }\end{array}$ & variable & $<250 \mathrm{ng}$ \\
\hline Template DNA & $1.0 \mu \mathrm{l}$ & \\
\hline $\begin{array}{l}\text { Phusion DNA polymerase } \\
\text { (New England BioLabs, USA) }\end{array}$ & to $50 \mu \mathrm{l}$ & \\
\hline Nuclease free water & & \\
\hline
\end{tabular}

Table 5: Standard PCR cycle condition

\begin{tabular}{|l|c|c|}
\hline \multicolumn{1}{|c|}{ Step } & Temperature & Time \\
\hline Initial denaturation & $98{ }^{\circ} \mathrm{C}$ & $3.00 \mathrm{~min}$ \\
\hline \multirow{3}{*}{$35-40$ cycles } & $98^{\circ} \mathrm{C}$ & $0.15 \mathrm{sec}$ \\
\cline { 2 - 3 } & depending on primer $\mathrm{T}_{\mathrm{m}}$ & $0.15 \mathrm{sec}$ \\
\cline { 2 - 3 } & $72{ }^{\circ} \mathrm{C}$ & $15-30 \mathrm{sec} / \mathrm{kb}$ \\
\hline Final extension & $72{ }^{\circ} \mathrm{C}$ & $10 \mathrm{~min}$ \\
\hline Hold & $20^{\circ} \mathrm{C}$ & \\
\hline
\end{tabular}




\subsubsection{Agarose gel electrophoresis}

DNA or RNA were resolved in an agarose gel with horizontal electric field (Sharp et al., 1973). Based on the expected DNA or RNA fragment size, Prepared $0.5-1 \%$ agarose gel in $1 \mathrm{X}$ TBE buffer 1x TBE buffer (90 mM Tris (pH 8.0), $90 \mathrm{mM}$ boric acid, $2 \mathrm{mM}$ EDTA) with $0.5 \mu \mathrm{g} / \mathrm{ml}$ ethidium bromide to visualize nucleic acids. Before loading on gel, DNA samples were mixed with 10X loading buffer (50 \% glycerol, $0.4 \%$ bromophenol blue) and RNA samples were mixed with gel loading buffer II (Life Technologies, Carlsbad, USA). Electrophoresis performed using $1 \mathrm{X}$ TBE buffer at 75-100 V. The $1 \mathrm{~kb}$ Plus DNA ladder (Life Technologies, Carlsbad, USA) was used to determine expected size of DNA or RNA fragments. Gels were documented using ChemiDoc gel documentation system (Bio-Rad, Munich).

\subsubsection{Purification of DNA}

DNA was purified from agarose gel or directly from PCR mixture itself using Gel and PCR clean-up kit according to the manufacturer's instructions. Concentration of purified DNA was determined with the NanoDrop 2000c spectrophotometer (Thermo Scientific, Wilmington, USA).

\subsubsection{Plasmid DNA preparation}

Pre-transformed Plasmid DNA was isolated from bacteria culture using the NucleoBond Xtra Midi Kit (Macherey and Nagel, Dueren) according to the manufacturer's instructions. The DNA concentration was was measured with the NanoDrop 2000c spectrophotometer (Thermo Scientific, Wilmington, USA).

\subsubsection{Gateway cloning}

The gateway cloning (Life Technologies, Carlsbad, USA) is developed based on the sitespecific recombination strategy used by bacteria phage $\lambda$ to integrate its DNA in the $E$. coli chromosome. There, specific recombination sites called attP in phage and attB in E. coli integrate each other to generate $a t t \mathrm{~L}$ and $a t t \mathrm{R}$ sites that flank the integrated phage DNA. 
First, designed forward and reverse primers containing attB site followed by gene specific sequence (Table 2). The attB-PCR products were recombined into an attP-site containing pDONR 221 vector in a standard gateway BP recombination reaction to generate gateway donor vectors (Table 5). Generated donor vector was recombined into appropriate destination vectors in standard gateway LR recombination reaction to generate expression clones (Table 6).

Table 6: Gateway BP recombination reaction

\begin{tabular}{|l|l|l|l|}
\hline Reaction Component & Sample & Negative control & Positive control \\
\hline attB-PCR products $(50-150 \mathrm{ng} / \mu \mathrm{l})$ & $1-7 \mu \mathrm{l}$ & $1-7 \mu \mathrm{l}$ & \\
\hline pDONR 221 vector $(150 \mathrm{ng} / \mu \mathrm{l})$ & $1.0 \mu \mathrm{l}$ & $1.0 \mu \mathrm{l}$ & $2.0 \mu \mathrm{l}$ \\
\hline pEXP7-tet positive control $(50 \mathrm{ng} / \mu \mathrm{l})$ & --- & --- & $2.0 \mu \mathrm{l}$ \\
\hline $5 X$ BP Clonase II Reaction Buffer & $2.0 \mu \mathrm{l}$ & $\mathbf{2 . 0} \boldsymbol{\mu l} \mathrm{H}_{\mathbf{2}} \mathbf{0}$ & $2.0 \mu \mathrm{l}$ \\
\hline Nuclease free water & to $10.0 \mu \mathrm{l}$ & to $10.0 \mu \mathrm{l}$ & $4.0 \mu \mathrm{l}$ \\
\hline
\end{tabular}

Table 7: Gateway LR recombination reaction

\begin{tabular}{|l|l|l|l|}
\hline Reaction Component & Sample & Negative control & Positive control \\
\hline pDONR Vector $(50-150 \mathrm{ng} / \mu \mathrm{l})$ & $1-7 \mu \mathrm{l}$ & $1-7 \mu \mathrm{l}$ & \\
\hline Destination Vector $(150 \mathrm{ng} / \mu \mathrm{l})^{*}$ & $1.0 \mu \mathrm{l}$ & $1.0 \mu \mathrm{l}$ & $2.0 \mu \mathrm{l}$ \\
\hline pENTR-gus positive control $(50 \mathrm{ng} / \mu \mathrm{l})$ & --- & ---- & $2.0 \mu \mathrm{l}$ \\
\hline $5 X$ LR Clonase II Reaction Buffer & $2.0 \mu \mathrm{l}$ & $\mathbf{2 . 0} \mu \mathrm{l} \mathrm{H20}$ & $2.0 \mu \mathrm{l}$ \\
\hline Nuclease free water & to $10.0 \mu \mathrm{l}$ & to $10.0 \mu \mathrm{l}$ & $4.0 \mu \mathrm{l}$ \\
\hline \multicolumn{5}{|l|}{ Gently mix and spin the reaction mixture, then incubate overnight at $25^{0} \mathrm{C}}$. \\
\hline
\end{tabular}




\subsubsection{In-Fusion cloning}

This is also based on site-specific recombination of gene of interest into a target vector. InFusion cloning primers were designed selecting one or two restriction enzymes from destination vector. In each case, both forward and reverse primers were containing $15 \mathrm{bp}$ overhangs in addition to the gene specific sequence. The destination vector was linearized using the same restriction enzymes used for the primer designing. PCR products was recombined into the linearized vector in a standard In-Fusion reaction as shown in table 7.

Table 8: In-Fusion cloning reaction

\begin{tabular}{|l|l|l|l|}
\hline Reaction Component & Sample & Negative control & Positive control \\
\hline PCR products $(100-200 \mathrm{ng} / \mu \mathrm{l})$ & $1-7 \mu \mathrm{l}$ & $1-7 \mu \mathrm{l}$ & $2 \mu \mathrm{l}$ of $2 \mathrm{~kb}$ control insert \\
\hline Linearized vector $(50-100 \mathrm{ng} / \mu \mathrm{l})$ & $1.0 \mu \mathrm{l}$ & $1.0 \mu \mathrm{l}$ & $1 \mu \mathrm{l}$ of pUC19 control vector \\
\hline $5 X$ In-Fusion HD enzyme premix & $2.0 \mu \mathrm{l}$ & $\mathbf{2 . 0} \boldsymbol{\mu l ~ \mathbf { H } _ { \mathbf { 2 } } \mathbf { 0 }}$ & $2.0 \mu \mathrm{l}$ \\
\hline Nuclease free water & to $10.0 \mu \mathrm{l}$ & to $10.0 \mu \mathrm{l}$ & $4.0 \mu \mathrm{l}$ \\
\hline \multicolumn{2}{|l}{ Gently mix and spin the reaction mixture. Then incubate at $\mathbf{5 0}{ }^{\circ} \mathbf{C}$ for $\mathbf{1 5}$ minutes. } \\
\hline
\end{tabular}

\subsubsection{Chemical transformation for gateway cloning}

Thawed and gently mixed $\mathrm{DH} 5 \alpha$ competent cells to ensure even distribution and aliquot 50 $\mu \mathrm{l}$ of the competent cells into a $1.5 \mathrm{ml}$ microcentrifuge tube. Added $2.5 \mu \mathrm{l}$ of the BP recombination reaction mixture to the cells and gently pipette the cells for even distribution of the reaction mixture. The cells were incubated on ice for 30 minutes followed by heatshock the competent cells for $45-60$ seconds at $42{ }^{\circ} \mathrm{C}$. Then cells were immediately transferred onto ice and kept for 2-3 minutes. After adding $450 \mu \mathrm{l}$ S.O.C medium $(0.5 \%$ Yeast Extract, 2\% Tryptone, $10 \mathrm{mM} \mathrm{NaCl}, 2.5 \mathrm{mM} \mathrm{KCl}, 10 \mathrm{mM} \mathrm{MgCl} 2,10 \mathrm{mM} \mathrm{MgSO}, 20 \mathrm{mM}$ Glucose) cells were incubated for one hour at $37{ }^{\circ} \mathrm{C}$ while horizontal shaking by $220 \mathrm{rpm}$. Spreaded $250 \mu \mathrm{l}$ of transformation reaction on a $1.5 \%$ LB-agar plate containing kanamycin (for BP recombination reaction) or ampicillin (LR recombination reaction) as a selection antibiotic and incubated the plates overnight at $37^{\circ} \mathrm{C}$. 


\subsubsection{Chemical transformation for In-Fusion cloning}

Performed same procedure described in section with few exception. Stellar ${ }^{\mathrm{TM}}$ competent cells were used instead of DH5 $\alpha$ competent cells. Plated $50-100 \mu$ l of sample on $1.5 \%$ LBagar plate containing ampicillin as a selection antibiotic and incubated the plates overnight at $37^{\circ} \mathrm{C}$.

\subsubsection{In vitro transcription}

Capped sense RNA was synthesized using the SP6 mMessage mMachine kit as described by the manufacturer (Life Technologies, Carlsbad, USA). A standard reaction was prepared as shown in the table.8. Synthesized RNA was purified with Illustra Probe Quant G-50 columns as described by the manufacturer (GE Healthcare, Little Chalfont, UK). The RNA concentration was determined with the NanoDrop 2000c spectrophotometer (Thermo Scientific, Wilmington, USA) and RNA integrity was determined by agarose gel electrophoresis

Table 9: In vitro transcription reaction set up

\begin{tabular}{|c|c|}
\hline Reaction component & Volume \\
\hline 2X NTP/ CAP & $10.0 \mu l$ \\
\hline 10X reaction buffer & $2.0 \mu l$ \\
\hline Linearized plasmid DNA & $100-1000 \mathrm{ng}$ \\
\hline SP6 RNA polymerase & $2.0 \mu \mathrm{l}$ \\
\hline Nuclease free water & To $20 \mu \mathrm{l}$ \\
\hline \multicolumn{2}{|c|}{ Gently mix the sample tube and spin the tube. } \\
\hline \multicolumn{2}{|c|}{ After incubate the sample at $42{ }^{\circ} \mathrm{C}$ for $2-3$ hours } \\
\hline After 3 hour & e sample for $15 \mathrm{~min}$ at $37^{\circ} \mathrm{C}$. \\
\hline
\end{tabular}




\subsubsection{SDS- polyacrylamide gel electrophoresis}

Zebrafish embryo cell lysate or in vitro translated proteins were resolved using SDSpolyacrylamide gel electrophoresis. Protein samples were mixed with 2x SDS loading buffer (100 mM Tris (pH 6.8), 20 \% glycerol, 4 \% SDS, 200 mM $\beta$-mercaptoethanol, $0.02 \%$ bromophenol blue), incubated for $5 \mathrm{~min}$ at $95^{\circ} \mathrm{C}$. Samples were loaded on $10-15 \%$ SDS gel based on the molecular weight of the target protein and the Page Ruler prestained protein ladder (Thermo Scientific, Wilmington, USA) was separately loaded to determine the approximate molecular weight of the proteins. Vertical electrophoresis performed in $1 x$ Laemmli buffer ( $25 \mathrm{mM}$ Tris, $250 \mathrm{mM}$ glycine, $0.01 \% \mathrm{SDS}$ ) at a constant voltage of $80 \mathrm{~V}$. After the dye front reached the seperating gel, the voltage was increased to $120 \mathrm{~V}$. Subsequently the gel was stained with Coomassie blue (section) or used the gel for western blotting.

\subsubsection{Coomassie staining}

After SDS-gel electrophoresis, the gels were washed with $\mathrm{dH}_{2} \mathrm{O}$. Thereafter, gels were incubated in Coomassie staining solution (50\% methanol, $10 \%$ glacial acetic acid, $0.1 \%$ Coomassie Brilliant Blue) for $30 \mathrm{~min}$ to $1 \mathrm{~h}$ at room temperature. The stained gels were washed three times with $\mathrm{dH}_{2} \mathrm{O}$ and incubated in a destaining solution (40\% methanol, $10 \%$ glacial acetic acid) over night at room temperature.

\subsubsection{Western blot}

After SDS-PAGE, proteins were transferred to a nitrocellulose membrane using semi-dry blotting. The nitrocellulose membrane and whatman papers were soaked in blotting buffer (39 mM glycine, $48 \mathrm{mM}$ Tris, 0.037 \% SDS, 20 \% methanol). Stacked three whatman papers and placed the nitrocellulose membrane on top of the whatman papers. Protein gel placed on the nitrocellulose membrane followed by stacking another whatman papers. The proteins were transferred for one hour at $250 \mathrm{~mA}$ and $25 \mathrm{~V}$. After, membrane was blocked using $5 \%$ skim milk in TBS (10 mM Tris (pH 8.0), $150 \mathrm{mM} \mathrm{NaCl)}$. The membrane incubated for one hour at $4{ }^{\circ} \mathrm{C}$. Then, the membrane was incubated overnight at $4{ }^{\circ} \mathrm{C}$ with diluted antibodies in $5 \%$ 
skim milk +TBST (10 mM Tris (pH 8.0), $150 \mathrm{mM} \mathrm{NaCl}, 0.05 \%$ Tween20). The membrane was washed $3 \times 5 \mathrm{~min}$ in TBST and incubated with fluorescent coupled secondary antibody for one hour at room temperature. The membrane was washed $3 \times 5$ min with TBST and fluorescent signal was detected using Odyssey CLx Infrared Imaging system (Li-Cor, Lincoln, USA) and analyzed with the Image Studio Software (Li-Cor, Lincoln, USA).

\subsubsection{Live-cell imaging}

Embryos were manually dechorionated and mounted on $1.5 \%$ agarose coated dishes filled with 1x E3 medium and imaged by stereo microscope SteREO Lumar.V12 (Carl Zeiss Microscopy, Jena), (Chapter 2.2.2). Images were processed using the software Axio Vision Rel. 4.8 (Carl Zeiss Microscopy, Jena). For imaging with LSM80, dechorionated embryos were placed on a Fluorodish (WPI, Sarasota, USA) with 1x E3. Images were analyzed using the ZEN 2011 software (Carl Zeiss Microscopy, Jena).

\subsubsection{Recombinant protein expression of Buc-VBM}

The Buc-VBM encoding amino acids 363-400 was amplified by PCR. The PCR product was cloned into pGEX-6p1 vector, which contains GST fusion tag using EcoRI restriction enzyme. The GST fusion protein was recombinantly expressed in BL21 (DE3) cells. The protein expression was induced by adding IPTG to $0.5 \mathrm{mM}$ final concentration and incubated overnight at $16^{\circ} \mathrm{C}$ while shaking at $200 \mathrm{rpm}$. Cells were harvested, resuspended and in lysis buffer disrupted using a microfluidizer (Microfluidics) in $50 \mathrm{mM} \mathrm{Tris/HCl} \mathrm{(pH} \mathrm{7.8),} 500 \mathrm{mM}$ $\mathrm{NaCl}, 5 \%(\mathrm{v} / \mathrm{v})$ glycerol and $10 \mathrm{mM}$ ethylenediaminetetraacetic acid (EDTA) + 1 protease inhibitor cOmplete ULTRA tablet/ $50 \mathrm{ml}$ buffer). The lysate was clarified by ultracentrifugation at $30000 \mathrm{~g}$ and $4{ }^{\circ} \mathrm{C}$ for 30 minutes. The clarified lysate loaded on Glutathion Sepharose column ( GE healthcare) at room temperature. The protein was subsequently eluted with $30 \mathrm{mM}$ reduced glutathione. The GST-tag was proteolytically cleaved by adding PreScission Protease ( $1 \mu \mathrm{l}$ ( 2 units) $100 \mu \mathrm{g}$ of fusion protein) in to the eluated protein and incubated overnight at $4{ }^{\circ} \mathrm{C}$. The tag was removed using a Superdex 75 gel-fltration column coupled to a Glutathione Sepharose column in $20 \mathrm{mM}$ Tris/ $\mathrm{HCl}(\mathrm{pH} 7.8)$, 
$200 \mathrm{mM} \mathrm{NaCl}, 5 \%$ glycerol and $2 \mathrm{mM} \mathrm{MgCl}_{2}$. The protein was concentrated Amicon Ultra centrifugal concentrator (Merck) and flash frozen in liquid nitrogen.

\subsubsection{Recombinant protein expression of Vasa (227-670) aa}

The Vasa encoding amino acids 227 -670 was amplified by PCR. The PCR product was cloned into pGEX-6p1 vector, which contains GST fusion tag using BamHI restriction enzyme. The GST fusion protein was recombinantly expressed in BL21 (DE3) cells. The protein expression was induced adding IPTG to $0.5 \mathrm{mM}$ final concentration together with Ethanol added $4 \%$ (V/V) , $\mathrm{K}_{2} \mathrm{HPO}_{4}$ to $30 \mathrm{mM}$ final concentration and incubated overnight at $16{ }^{\circ} \mathrm{C}$ while shaking at $200 \mathrm{rpm}$. Cells were harvested, resuspended and in lysis buffer disrupted using a microfluidizer (Microfluidics) in $50 \mathrm{mM}$ Tris/ $\mathrm{HCl}$ (pH 7.8), $500 \mathrm{mM} \mathrm{NaCl}, 5 \%(\mathrm{v} / \mathrm{v})$ glycerol and $10 \mathrm{mM}$ ethylenediaminetetraacetic acid (EDTA) + 1 protease inhibitor cOmplete ULTRA tablet/ $50 \mathrm{ml}$ buffer). The lysate was clarified by ultracentrifugation at $30000 \mathrm{~g}$ and $4{ }^{\circ} \mathrm{C}$ for 30 minutes. The clarified lysate loaded on Glutathion Sepharose column ( GE healthcare) at room temperature. Potentially bound nucleic acids were removed washing the lysate with was buffer supplemented with $2 \mathrm{M} \mathrm{LiCl}$. The protein was subsequently eluted with $30 \mathrm{mM}$ reduced glutathione. The GST-tag was proteolytically cleaved by adding PreScission Protease ( $1 \mu \mathrm{l}$ ( 2 units) $100 \mu \mathrm{g}$ of fusion protein) in to the eluated protein and incubated overnight at $4^{\circ} \mathrm{C}$. The tag was removed using a Superdex 75 gel-fltration column coupled to a Glutathione Sepharose column in $20 \mathrm{mM}$ Tris/ $\mathrm{HCl}$ (pH 7.8), $200 \mathrm{mM} \mathrm{NaCl}, 5 \%$ glycerol and 2 $\mathrm{mM} \mathrm{MgCl}$. The protein was concentrated Amicon Ultra centrifugal concentrator (Merck) and flash frozen in liquid nitrogen. 


\subsubsection{ATPase assay}

The ATPase activity of Vasa was measured with a nicotinamide adenine dinucleotide (NADH) dependent coupled enzymatic assay (Kiianitsa et al., 2003) The assay detects the reduction of the $\mathrm{NADH}$ absorption at $340 \mathrm{~nm}$ as a direct effect of the ATP consumption over time with a VICTOR Nivo Multimode Microplate Reader (PerkinElmer). The ATPase activity of Vasa was determined by mixing $5 \mu \mathrm{M}$ Vasa with and without $100 \mu \mathrm{M}$ of Buc-VBM together with $2.5 \mathrm{mM}, 250 \mathrm{nM}$ NADH, $500 \mathrm{nM}$ phosphoenolpyruvate, 6-8.3 U ml-1 pyruvate kinase and 9-14 U ml-1 lactic dehydrogenase. RNA stimulated ATPase activity of Vasa was performed by adding $50 \mu \mathrm{M}$ ssRNA (polyA $(8)$ ) into $5 \mu \mathrm{M}$ Vasa with and without $100 \mu \mathrm{M}$ of Buc-VBM with other common components listed. All reactions were performed in triplicates of $150 \mu \mathrm{l}$ in each at $25^{\circ} \mathrm{C}$.

\subsubsection{Circular dichroism (CD) spectroscopy}

CD-spectra for Buc-VBM (amino acid 363-400) was performed using a Chirascan CD spectrometer (Applied Photophysics) at far-UV spectra (185-260 nm). Initially, protein buffer was exchanged to a buffer containing $20 \mathrm{mM}$ Na-phosphate buffer to favor the CD-spectra. Measurements were recorded at room temperature using $0.1 \mathrm{mg} / \mathrm{ml}$ Buc-VBM protein concentration. The CD data are presented as molar ellipticity $\left([\theta] \operatorname{deg} \times \mathrm{cm}^{2} / \mathrm{dmol}\right.$ and plotted using QtiPlot (v.0.9.8.9).

\subsection{Bioinformatics methods}

\subsubsection{Pairwise sequence alignment}

Sequences were pairwise aligned using Needleman-Wunsch algorithm (https://www.ebi.ac.uk/Tools/psa/emboss needle/) .

\subsubsection{Multiple sequence alignments}

Multiple sequence alignment was performed using T-Coffee multiple sequence alignment server (https://www.ebi.ac.uk/Tools/msa/tcoffee/) (Notredame et al., 2000). 
4.5.3. Aligns protein sequences using structural information

Protein sequences were aligned using T-Coffee Expresso server (http://tcoffee.crg.cat/apps/tcoffee/do:expresso) (Di Tommaso et al., 2011).

\subsubsection{In silico protein modeling}

PyMol Ve 2.3 was used to visualize and structural arrangement and alignment of predicted protein models. 


\section{References}

Anne, J. (2010). Arginine methylation of SmB is required for Drosophila germ cell development. Development. 137 (17). p.pp. 2819-2828.

Ashok Kumar, T. (2013). CFSSP: Chou and Fasman Secondary Structure Prediction server. Wide Spectrum. 1 (9). p.pp. 15-19.

Bayer, P., Arndt, A., Metzger, S., Mahajan, R., Melchior, F., Jaenicke, R. \& Becker, J. (1998). Structure determination of the small ubiquitin-related modifier SUMO-1. Journal of Molecular Biology. 280 (2). p.pp. 275-286.

Bolger, T.A. \& Wente, S.R. (2011). Gle1 is a multifunctional DEAD-box protein regulator that modulates Ded1 in translation initiation. Journal of Biological Chemistry. 286 (46). p.pp. 39750-39759.

Bontems, F., Stein, A., Marlow, F., Lyautey, J., Gupta, T., Mullins, M.C. \& Dosch, R. (2009). Bucky Ball Organizes Germ Plasm Assembly in Zebrafish. Current Biology. [Online]. 19 (5). p.pp. 414-422. Available from:

http://dx.doi.org/10.1016/j.cub.2009.01.038.

Boswell, R.E. \& Mahowald, A.P. (1985). tudor, a gene required for assembly of the germ plasm in Drosophila melanogaster. Cell. 43 (1). p.pp. 97-104.

Braat, A.K., Zandbergen, T., Van De Water, S., Goos, H.J.T.H. \& Zivkovic, D. (1999). Characterization of zebrafish primordial germ cells: Morphology and early distribution of vasa RNA. Developmental Dynamics. 216 (2). p.pp. 153-167.

Breitwieser, W., Markussen, F.H., Horstmann, H. \& Ephrussi, A. (1996). Oskar protein interaction with vasa represents an essential step in polar granule 
assembly. Genes and Development. 10 (17). p.pp. 2179-2188.

Buehr, M.L. \& Blackler, A.W. (1970). Sterility and partial sterility in the South African clawed toad following the pricking of the egg. Journal of Embryology and Experimental Morphology. 23 (2). p.pp. 375-384.

Carr, R. M., Oranu, A., \& Khungar, V. (2016). piRNA Biogenesis in Drosophila Melanogaster. Physiology \& behavior. 176 (1). p.pp. 139-148.

Caruthers, J.M. \& McKay, D.B. (2002). Helicase structure and mechanism. Current Opinion in Structural Biology. 12 (1). p.pp. 123-133.

Caussinus, E., Kanca, O. \& Affolter, M. (2012). Fluorescent fusion protein knockout mediated by anti-GFP nanobody. Nature Structural and Molecular Biology. 19 (1). p.pp. 117-122.

Cieri, D., Vicario, M., Giacomello, M., Vallese, F., Filadi, R., Wagner, T., Pozzan, T., Pizzo, P., Scorrano, L., Brini, M. \& Calì, T. (2018). SPLICS: A split green fluorescent protein-based contact site sensor for narrow and wide heterotypic organelle juxtaposition. Cell Death and Differentiation. 25 (6). p.pp. 1131-1145.

Clift, D., So, C., McEwan, W.A., James, L.C. \& Schuh, M. (2018). Acute and rapid degradation of endogenous proteins by Trim-Away. Nature Protocols. [Online]. 13 (10). p.pp. 2149-2175. Available from: http://dx.doi.org/10.1038/s41596-018-0028-3.

Cole, C., Barber, J.D. \& Barton, G.J. (2008). The Jpred 3 secondary structure prediction server. Nucleic acids research. 36 (Web Server issue). p.pp. 197201. 
Cusanovich, D.A., Pavlovic, B., Pritchard, J.K. \& Gilad, Y. (2014). The Functional Consequences of Variation in Transcription Factor Binding. PLOS Genetics. $10(3)$.

Dahanukar, A., Walker, J.A. \& Wharton, R.P. (1999). Smaug, a novel RNAbinding protein that operates a translational switch in Drosophila. Molecular Cell. 4 (2). p.pp. 209-218.

Dosch, R. (2015). Next generation mothers: Maternal control of germline development in zebrafish. Critical Reviews in Biochemistry and Molecular Biology. 50 (1). p.pp. 54-68.

Dosch, R., Wagner, D.S., Mintzer, K.A., Runke, G., Wiemelt, A.P. \& Mullins, M.C. (2004). Maternal control of vertebrate development before the midblastula transition: Mutants from the zebrafish I. Developmental Cell. 6 (6). p.pp. 771-780.

Drozdetskiy, A., Cole, C., Procter, J. \& Barton, G.J. (2015). JPred4: A protein secondary structure prediction server. Nucleic Acids Research. 43 (W1). p.pp. W389-W394.

Dudgeon, C.L., Coulton, L., Bone, R., Ovenden, J.R. \& Thomas, S. (2017). Switch from sexual to parthenogenetic reproduction in a zebra shark. Scientific Reports. 7 (January). p.pp. 1-8.

Durdevic, Z. \& Ephrussi, A. (2019). Germ cell lineage homeostasis in drosophila requires the vasa RNA helicase. Genetics. 213 (3). p.pp. 911-922.

Ephrussi, Anne; Lehmann, R. (1992). Induction of germ cell fate by oskar. Nature. 358 (July). p.pp. 387-392. 
Ephrussi, A., Dickinson, L.K. \& Lehmann, R. (1991). Oskar Organizes the Germ Plasm and Directs Localization of the Posterior Determinant Nanos. Cell. 66 (1). p.pp. 37-50.

Extavour, C.G. \& Akam, M. (2003). Mechanisms of germ cell specification across the metazoans: Epigenesis and preformation. Development. 130 (24). p.pp. 5869-5884.

Florence L. Marlow and Mary C. Mullins (2008). Bucky ball functions in Balbiani body assembly and animal- vegetal polarity in the oocyte and follicle cell layer in zebrafish. Developmental Biology. 321 (1). p.pp. 40-50.

Gustafson, E.A. \& Wessel, G.M. (2010). Vasa genes: Emerging roles in the germ line and in multipotent cells. BioEssays. 32 (7). p.pp. 626-637.

Hamann, F., Enders, M. \& Ficner, R. (2019). Structural basis for RNA translocation by DEAH-box ATPases. Nucleic Acids Research. 47 (8). p.pp. 4349-4362.

Hashimoto, Y., Maegawa, S., Nagai, T., Yamaha, E., Suzuki, H., Yasuda, K. \& Inoue, K. (2004). Localized maternal factors are required for zebrafish germ cell formation. Developmental Biology. 268 (1). p.pp. 152-161.

Heim, A.E., Hartung, O., Rothhämel, S., Ferreira, E., Jenny, A. \& Marlow, F.L. (2014). Oocyte polarity requires a Bucky ball-dependent feedback amplification loop. Development (Cambridge). 141 (4). p.pp. 842-854.

Hickford, D.E., Frankenberg, S., Pask, A.J., Shaw, G. \& Renfree, M.B. (2011). DDX4 (VASA) Is Conserved in Germ Cell Development in Marsupials and Monotremes1. Biology of Reproduction. 85 (4). p.pp. 733-743. 
Ikenishi, K., Kotani, M. \& Tanabe, K. (1974). Ultrastructural changes associated with UV irradiation in the 'germinal plasm' of Xenopus laevis. Developmental Biology. 36 (1). p.pp. 155-168.

Ikenishi, K., Sakiko, N. \& Okuda, T. (1986). Direct Evidence for the Presence of Germ Cell Determinant in Vegetal Pole Cytoplasm of Xenopus laevis and in a Subcellular Fraction of It: (Xenopus laevis/germ cell determinant/germ plasm/PGC induction). Development, Growth \& Differentiation. 28 (6). p.pp. 563-568.

Illmensee, K. \& Mahowald, A.P. (1974). Transplantation of posterior polar plasm in Drosophila. Induction of germ cells at the anterior pole of the egg. Proceedings of the National Academy of Sciences of the United States of America. 71 (4). p.pp. 1016-1020.

Jankowsky, E. (2011). RNA helicases at work: Binding and rearranging. Trends in Biochemical Sciences. [Online]. 36 (1). p.pp. 19-29. Available from: http://dx.doi.org/10.1016/j.tibs.2010.07.008.

Jensen, L.J., Ussery, D.W. \& Brunak, S. (2003). Functionality of system components: Conservation of protein function in protein feature space. Genome Research. 13 (11). p.pp. 2444-2449.

Jeske, M., Bordi, M., Glatt, S., Müller, S., Rybin, V., Müller, C.W. \& Ephrussi, A. (2015). The crystal structure of the Drosophila germline inducer Oskar identifies two domains with distinct Vasa Helicase- and RNA-binding activities. Cell Reports. 12 (4). p.pp. 587-598.

Jeske, M., Müller, C.W. \& Ephrussi, A. (2017). The LOTUS domain is a conserved DEAD-box RNA helicase regulator essential for the recruitment of Vasa to the germ plasm and nuage. Genes and Development. 31 (9). p.pp. 939-952. 
Johnstone, O. \& Lasko, P. (2004). Interaction with elF5B is essential for Vasa function during development. Development. 131 (17). p.pp. 4167-4178.

Jostes, S. \& Schorle, H. (2018). Signals and transcription factors for specification of human germ cells. Stem Cell Investigation. 5 (3). p.pp. 1-5.

Juliano, C.E., Swartz, S.Z. \& Wessel, G.M. (2010). A conserved germline multipotency program. Development. 137 (24). p.pp. 4113-4126.

Kaczka, P., Winiewska, M., Zhukov, I., Rempoła, B., Bolewska, K., Łozinski, T., Ejchart, A., Poznańska, A., Wierzchowski, K.L. \& Poznański, J. (2014). The TFE- induced transient native- like structure of the intrinsically disordered $\sigma \_4^{\wedge} 704$ domain of Escherichia coli RNA polymerase. European Biophysics Journal. 43 (12). p.pp. 581-594.

Kerppola, T.K. (2008). Bimolecular fluorescence complementation (BiFC) analysis as a probe of protein interactions in living cells. Annu Rev Biophys. 37. p.pp. $465-87$.

Kerppola, T.K. (2006). Design and implementation of bimolecular fluorescence complementation (BiFC) assays for the visualization of protein interactions in living cells. Nature Protocols. 1 (3). p.pp. 1278-1286.

Kerppola, T.K. (2006). Visualization of molecular interactions by fluorescence complementation. Nature Reviews Molecular Cell Biology. 7 (6). p.pp. 449456.

Kiianitsa, K., Solinger, J.A. \& Heyer, W.D. (2003). NADH-coupled microplate photometric assay for kinetic studies of ATP-hydrolyzing enzymes with low and high specific activities. Analytical Biochemistry. 321 (2). p.pp. 266-271. 
Kim-Ha, J., Smith, J.L. \& Macdonald, P.M. (1991). oskar mRNA is localized to the posterior pole of the Drosophila oocyte. Cell. 66 (1). p.pp. 23-35.

Kirino, Y., Vourekas, A., Kim, N., De Lima Alves, F., Rappsilber, J., Klein, P.S., Jongens, T.A. \& Mourelatos, Z. (2010). Arginine methylation of vasa protein is conserved across phyla. Journal of Biological Chemistry. 285 (11). p.pp. 8148-8154.

Kloc, M., Bilinski, S. \& Etkin, L.D. (2004). The Balbiani Body and Germ Cell Determinants: 150 Years Later. Current Topics in Developmental Biology. 59. p.pp. 1-36.

Kloc, M., Jedrzejowska, I., Tworzydlo, W. \& Bilinski, S.M. (2014). Balbiani body, nuage and sponge bodies - The germ plasm pathway players. Arthropod Structure and Development. [Online]. 43 (4). p.pp. 341-348. Available from: http://dx.doi.org/10.1016/j.asd.2013.12.003.

Köprunner, M., Thisse, C., Thisse, B. \& Raz, E. (2001). A zebrafish nanos-related gene is essential for the development of primordial germ cells. Genes and Development. 15 (21). p.pp. 2877-2885.

Krishnakumar, P. \& Dosch, R. (2018). Germ Cell Specification: The Evolution of a Recipe to Make Germ Cells. In: Germ Cell. pp. 1-22.

Krishnakumar, P., Riemer, S., Perera, R., Lingner, T., Goloborodko, A., Khalifa, H., Bontems, F., Kaufholz, F., El-Brolosy, M.A. \& Dosch, R. (2018). Functional equivalence of germ plasm organizers. PLoS Genetics. 14 (11). p.pp. 1-29. 
Kwan, K.M., Fujimoto, E., Grabher, C., Mangum, B.D., Hardy, M.E., Campbell, D.S., Parant, J.M., Yost, H.J., Kanki, J.P. \& Chien, C. Bin (2007). The Tol2kit: A multisite gateway-based construction Kit for Tol2 transposon transgenesis constructs. Developmental Dynamics. 236 (11). p.pp. 30883099.

Lasko, P.F. \& Ashburner, M. (1988). The product of the Drosophila gene vasa is very similar to eukaryotic initiation factor-4A. Nature. 335 (6191). p.pp. $611-617$.

Laureto, P.P. De, Donadi, M., Scaramella, E., Frare, E. \& Fontana, A. (2001). Trifluoroethanol-assisted protein folding: fragment 53-103 of bovine $\alpha-$ lactalbumin. Biosystems. 1548. p.pp. 29-37.

Lavial, F., Acloque, H., Bachelard, E., Nieto, M.A., Samarut, J. \& Pain, B. (2009). Ectopic expression of Cvh (Chicken Vasa homologue) mediates the reprogramming of chicken embryonic stem cells to a germ cell fate. Developmental Biology. [Online]. 330 (1). p.pp. 73-82. Available from: http://dx.doi.org/10.1016/j.ydbio.2009.03.012.

Lebaron, S., Papin, C., Capeyrou, R., Chen, Y.L., Froment, C., Monsarrat, B., Caizergues-Ferrer, M., Grigoriev, M. \& Henry, Y. (2009). The ATPase and helicase activities of Prp43p are stimulated by the G-patch protein Pfa1p during yeast ribosome biogenesis. EMBO Journal. 28 (24). p.pp. 38083819.

Lehmann, R. \& Nüsslein-Volhard, C. (1986). Abdominal segmentation, pole cell formation, and embryonic polarity require the localized activity of oskar, a maternal gene in drosophila. Cell. 47 (1). p.pp. 141-152. 
Liang, L., Diehl-Jones, W. \& Lasko, P. (1994). Localization of vasa protein to the Drosophila pole plasm is independent of its RNA-binding and helicase activities. Development. 120 (5). p.pp. 1201-1211.

Linder, P. (2006). Dead-box proteins: A family affair - Active and passive players in RNP-remodeling. Nucleic Acids Research. 34 (15). p.pp. 4168-4180.

Linder, P. \& Jankowsky, E. (2011). From unwinding to clamping - the DEAD box RNA helicase family. Nature Reviews Molecular Cell Biology. [Online]. 12 (8). p.pp. 505-516. Available from: http://dx.doi.org/10.1038/nrm3154.

Linder, P. \& Lasko, P. (2006). Bent out of Shape: RNA Unwinding by the DEADBox Helicase Vasa. Cell. 125 (2). p.pp. 219-221.

Linding, R., Schymkowitz, J., Rousseau, F., Diella, F. \& Serrano, L. (2004). A comparative study of the relationship between protein structure and $\beta$ aggregation in globular and intrinsically disordered proteins. Journal of Molecular Biology. 342 (1). p.pp. 345-353.

Liu, N., Han, H. \& Lasko, P. (2009). Vasa promotes Drosophila germline stem cell differentiation by activating mei-P26 translation by directly interacting with a (U)-rich motif in its 3' UTR. Genes and Development. 23 (23). p.pp. 27422752.

Marlow, F. (2015). Primordial Germ Cell Specification and Migration. F1000Research. 4 (0). p.pp. 1-14.

Marlow, F.L. (2010). Maternal Control of Development in Vertebrates.

Miles, L.B. \& Verkade, H. (2014). TA-cloning vectors for rapid and cheap cloning of zebrafish transgenesis constructs. Zebrafish. 11 (3). p.pp. 281-282. 
Miller, K.E., Kim, Y., Huh, W., Park, H., Developmental, C. \& Program, B. (2016). Bimolecular fluorescence complementation (BiFC) analysis: advances and recent applications for genome-wide interaction studies. 427 (11). p.pp. 2039-2055.

Nakamura, S., Hira, S., Fujiwara, M., Miyagata, N., Tsuji, T., Kondo, A., Kimura, H., Shinozuka, Y., Hayashi, M., Kobayashi, S. \& Mukai, M. (2019). A truncated form of a transcription factor Mamo activates vasa in Drosophila embryos. Communications Biology. [Online]. 2 (1). p.pp. 1-13. Available from: http://dx.doi.org/10.1038/s42003-019-0663-4.

Nakamura, T. \& Extavour, C.G. (2016). The transcriptional repressor Blimp-1 acts downstream of BMP signaling to generate primordial germ cells in the cricket gryllus bimaculatus. Development (Cambridge). 143 (2). p.pp. 255263.

Norma J. Greenfield (2012). Using circular dichroism spectra to estimate protein secondary structure. ProQuest Dissertations and Theses. 1 (6). p.p. 218.

Notredame, C., Higgins, D.G. \& Heringa, J. (2000). T-coffee: A novel method for fast and accurate multiple sequence alignment. Journal of Molecular Biology. 302 (1). p.pp. 205-217.

O.H. Kaufman and F.L. Marlow (2017). Methods to study maternal regulation of germ cell specification in zebrafish. Physiology \& behavior. 176 (5). p.pp. 139-148.

Pahlich, S., Zakaryan, R.P. \& Gehring, H. (2006). Protein arginine methylation: Cellular functions and methods of analysis. Biochimica et Biophysica Acta Proteins and Proteomics. 1764 (12). p.pp. 1890-1903. 
Pek, J.W., Patil, V.S. \& Kai, T. (2012). piRNA pathway and the potential processing site, the nuage, in the Drosophila germline. Development Growth and Differentiation. 54 (1). p.pp. 66-77.

Popovic, M., Bialecka, M., Gomes Fernandes, M., Taelman, J., Van Der Jeught, M., De Sutter, P., Heindryckx, B. \& De Sousa Lopes, S.M.C. (2019). Human blastocyst outgrowths recapitulate primordial germ cell specification events. Molecular Human Reproduction. 25 (9). p.pp. 519-526.

Raz, E. (2003). Primordial germ-cell development: The zebrafish perspective. Nature Reviews Genetics. 4 (9). p.pp. 690-700.

Raz, E. (2000). The function and regulation of vasa-like genes in germ-cell development. Genome biology. 1 (3). p.pp. 1-6.

Riemer, S., Bontems, F., Krishnakumar, P., Gömann, J. \& Dosch, R. (2015). A functional Bucky ball-GFP transgene visualizes germ plasm in living zebrafish. Gene Expression Patterns. [Online]. 18 (1-2). p.pp. 44-52. Available from: http://dx.doi.org/10.1016/j.gep.2015.05.003.

Rogers, G.W., Richter, N.J., Lima, W.F. \& Merrick, W.C. (2001). Modulation of the Helicase Activity of elF4A by elF4B, elF4H, and elF4F. Journal of Biological Chemistry. 276 (33). p.pp. 30914-30922.

Roovers, E.F., Kaaij, L.J.T., Redl, S., Bronkhorst, A.W., Wiebrands, K., de Jesus Domingues, A.M., Huang, H.Y., Han, C.T., Riemer, S., Dosch, R., Salvenmoser, W., Grün, D., Butter, F., van Oudenaarden, A. \& Ketting, R.F. (2018). Tdrd6a Regulates the Aggregation of Buc into Functional Subcellular Compartments that Drive Germ Cell Specification. Developmental Cell. 46 (3). p.pp. 285-301.e9. 
Roy, A., Kucukural, A. \& Zhang, Y. (2010). I-TASSER: A unified platform for automated protein structure and function prediction. Nature Protocols. 5 (4). p.pp. 725-738.

Saitou, M. \& Yamaji, M. (2010). Germ cell specification in mice: Signaling, transcription regulation, and epigenetic consequences. Reproduction. 139 (6). p.pp. 931-942.

Santos, A.C. \& Lehmann, R. (2004). Germ cell specification and migration in Drosophila and beyond. Current Biology. 14 (14). p.pp. 578-589.

Schupbach, T. \& Wieschaus, E. (1986). Germline autonomy of maternal-effect mutations altering the embryonic body pattern of Drosophila. Developmental Biology. 113 (2). p.pp. 443-448.

Schüpbach, T. \& Wieschaus, E. (1986). Maternal-effect mutations altering the anterior-posterior pattern of the Drosophila embryo. Roux's Archives of Developmental Biology. 195 (5). p.pp. 302-317.

Sengoku, T., Nureki, O., Nakamura, A., Kobayashi, S. \& Yokoyama, S. (2006). Structural Basis for RNA Unwinding by the DEAD-Box Protein Drosophila Vasa. Cell. 125 (2). p.pp. 287-300.

Singh, H., Singh, S. \& Singh Raghava, G.P. (2019). Peptide Secondary Structure Prediction using Evolutionary Information. bioRxiv. [Online]. p.p. 558791. Available from:

http://biorxiv.org/content/early/2019/02/22/558791.abstract.

Sloan, K.E. \& Bohnsack, M.T. (2018). Unravelling the Mechanisms of RNA Helicase Regulation. Trends in Biochemical Sciences. [Online]. 43 (4). p.pp. 237-250. Available from: https://doi.org/10.1016/j.tibs.2018.02.001. 
Smith, J.L., Wilson, J.E. \& Macdonald, P.M. (1992). Overexpression of oskar directs ectopic activation of nanos and presumptive pole cell formation in Drosophila embryos. Cell. 70 (5). p.pp. 849-859.

Smith, L.D. (1966). The role of a 'germinal plasm' in the formation of primordial germ cells in Rana pipiens. Developmental Biology. 14 (2). p.pp. 330-347.

St Johnston, D., Beuchle, D. \& Nüsslein-Volhard, C. (1991). staufen, a gene required to localize maternal RNAs in the Drosophila egg. Cell. 66 (1). p.pp. $51-63$.

Styhler, S., Nakamura, A., Swan, A., Suter, B. \& Lasko, P. (1998). vasa is required for GURKEN accumulation in the oocyte, and is involved in oocyte differentiation and germline cyst development. Development. 125 (9). p.pp. $1569-1578$.

Tada, H., Mochii, M., Orii, H. \& Watanabe, K. (2012). Ectopic formation of primordial germ cells by transplantation of the germ plasm: Direct evidence for germ cell determinant in Xenopus. Developmental Biology. [Online]. 371 (1). p.pp. 86-93. Available from:

http://dx.doi.org/10.1016/j.ydbio.2012.08.014.

Tanabe, K. \& Kotani, M. (1974). Relationship between the amount of the 'germinal plasm' and the number of primordial germ cells in Xenopus laevis. Journal of Embryology and Experimental Morphology. 31 (1). p.pp. 89-98.

Tomancak, P., Guichet, A., Zavorszky, P. \& Ephrussi, A. (1998). Oocyte polarity depends on regulation of gurken by Vasa. Development. 125 (9). p.pp. 1723-1732. 
Di Tommaso, P., Moretti, S., Xenarios, I., Orobitg, M., Montanyola, A., Chang, J.M., Taly, J.F. \& Notredame, C. (2011). T-Coffee: A web server for the multiple sequence alignment of protein and RNA sequences using structural information and homology extension. Nucleic Acids Research. 39 (SUPPL. 2). p.pp. 13-17.

Vanzo, N.F. \& Ephrussi, A. (2002). Oskar anchoring restricts pole plasm formation to the posterior of the Drosophila oocyte. Development. 129 (15). p.pp. 3705-3714.

Villefranc, J.A., Amigo, J. \& Lawson, N.D. (2007). Gateway compatible vectors for analysis of gene function in the zebrafish. Developmental Dynamics. 236 (11). p.pp. 3077-3087.

Vourekas, A., Kirino, Y. \& Mourelatos, Z. (2010). Elective affinities: A TudorAubergine tale of germline partnership. Genes and Development. 24 (18). p.pp. $1963-1966$.

Wagner, D.S., Dosch, R., Mintzer, K.A., Wiemelt, A.P. \& Mullins, M.C. (2004). Maternal control of development at the midblastula transition and beyond: Mutants from the zebrafish II. Developmental Cell. 6 (6). p.pp. 781-790.

Wakahara, M. (1977). Partial characterization of 'primordial germ cell forming activity' localized in vegetal pole cytoplasm in anuran eggs. Journal of Embryology and Experimental Morphology. Vol. 39. p.pp. 221-233.

Wake, D.B., Wake, M.H. \& Specht, C.D. (2011). Homoplasy: From detecting pattern to determining process and mechanism of evolution. Science. 331 (6020). p.pp. 1032-1035.

Walgers, R., Lee, T.C. \& Cammers-Goodwin, A. (1998). An indirect chaotropic mechanism for the stabilization of helix conformation of peptides in 
aqueous trifluoroethanol and hexafluoro-2- propanol. Journal of the American Chemical Society. 120 (20). p.pp. 5073-5079.

Warkocki, Z., Schneider, C., Mozaffari-Jovin, S., Schmitzová, J., Höbartner, C., Fabrizio, P. \& Lührmann, R. (2015). The G-patch protein Spp2 couples the spliceosome-stimulated ATpase activity of the deah-box protein Prp2 to catalytic activation of the spliceosome. Genes and Development. 29 (1). p.pp. 94-107.

Xiol, J., Spinelli, P., Laussmann, M.A., Homolka, D., Yang, Z., Cora, E., Couté, Y., Conn, S., Kadlec, J., Sachidanandam, R., Kaksonen, M., Cusack, S., Ephrussi, A. \& Pillai, R.S. (2014). RNA clamping by Vasa assembles a piRNA amplifier complex on transposon transcripts. Cell. 157 (7). p.pp. 1698-1711.

Yoon, C., Kawakami, K. \& Hopkins, N. (1997). Zebrafish vasa homologue RNA is localized to the cleavage planes of 2- and 4-cell-stage embryos and is expressed in the primordial germ cells. Development. 124 (16). p.pp. 31573165.

Zhu, J., Zhang, D., Liu, X., Yu, G., Cai, X., Xu, C., Rong, F., Ouyang, G., Wang, J. \& Xiao, W. (2019). Zebrafish prmt5 arginine methyltransferase is essential for germ cell development. Development (Cambridge). 146 (20). 


\section{List of Figures}

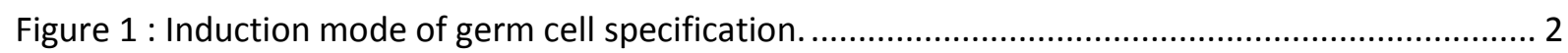

Figure 2: Inherited mode of germ cell specification. .................................................................... 2

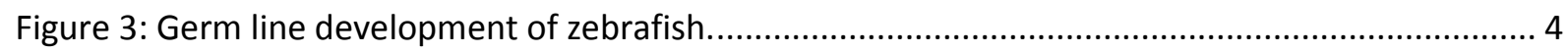

Figure 4: Drosophila Oskar is sufficient and necessary for germ cell formation. ................................. 6

Figure 5: Buc is required to organize germ plasm in zebrafish. .......................................................... 7

Figure 6: Phenotype of buc wild-type and buc mutant embryos...................................................... 8

Figure 7: Schematic representation of evolutionary conserved motifs in Buc. ................................... 9

Figure 8: Anti-Buc antibody specifically recognize Buc..................................................................... 9

Figure 9: Dynamic of Buc localization during oogenesis ................................................................. 10

Figure 10: Buc-GFP transgenic embryos mimics the dynamics of germ plasm localization. ................ 11

Figure 11: Schematic illustration of germ cell induction assay....................................................... 12

Figure 12: Buc and Oskar in germ cell induction assay. ................................................................... 13

Figure 13: Schematic illustration of general features of DEAD box RNA helicase. ............................. 15

Figure 14: Buc interacts with Vasa during germ cell specification..................................................... 17

Figure 15: Vasa induce ectopic germ cell in zebrafish. .................................................................. 18

Figure 16: Buc and Oskar are intrinsically disordered proteins. .................................................... 19

Figure 17: Schematic illustration of bimolecular fluorescence complementation assay (BiFC) assay. 22

Figure 18: Buc and Vasa interact in vivo during germ cell specification. ........................................... 23

Figure 19: Expression Vector maps and key core elements of BiFC gateway destination vectors....... 24

Figure 20: Identification of Buc-Vasa binding motif (Buc-VBM). ...................................................... 26

Figure 21: The Vasa-Buc binding motif (Vasa-BBM) is located in C-terminal region of Vasa. ............... 28

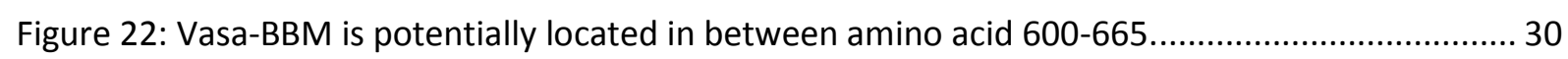

Figure 23: Vasa-BBM is located in amino acids 600-625.............................................................. 31

Figure 24: Buc-VBM and Vasa-BBM are required for Buc and Vasa interaction................................. 32

Figure 25: Buc $\triangle V B M-G F P$ and Vasa $\triangle B B M$ are expressed in vivo....................................................... 33

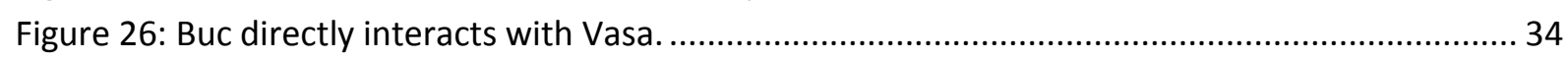

Figure 27: Pairwise amino acid sequence alignment of Drosophila and zebrafish Vasa. ..................... 35

Figure 28: Alignment of a predicted zebrafish Vasa structure with the structure solved for Drosophila

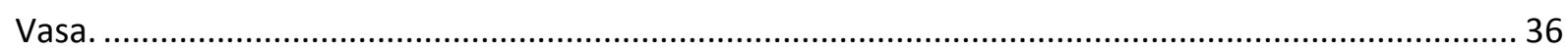

Figure 29: Illustration of Drosophila Vasa and Oskar LOTUS domain binding interface........................ 38

Figure 30: C-terminal extension of Drosophila Oskar LOTUS domain (eLOTUS) adopts an $\alpha$-helix

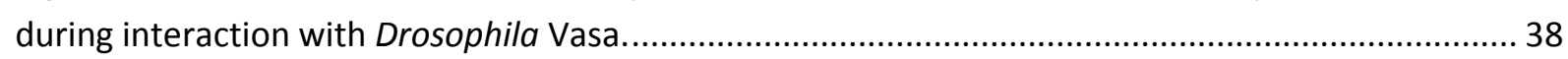

Figure 31: In silico secondary structure prediction for Buc-VBM................................................... 40

Figure 32: Secondary structure prediction for Buc-VBM (amino acid 363-400) using CD-spectroscopy.

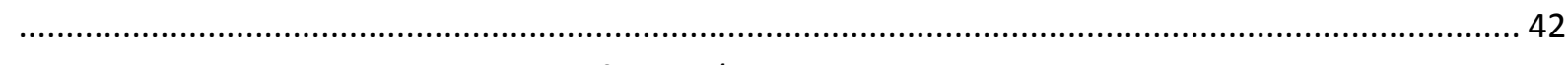

Figure 33: Schematic representation of NADH/LDH coupled ATPase assay......................................... 44

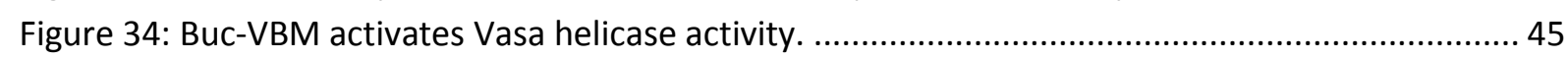

Figure 35: Buc-Vasa binding motifs and its intrinsically disordered regions are not sufficient for germ

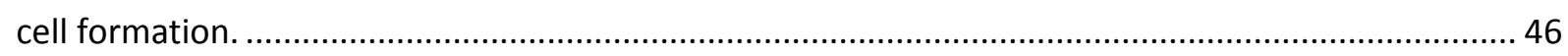

Figure 36: Buc-GFP larvae showed less germ cell after injection of Buc-VBM and Vasa-BBM into Buc-

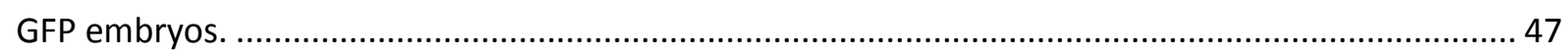

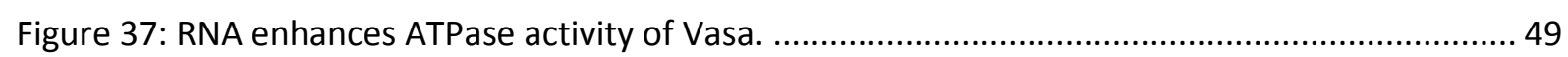


Figure 38: Alignment of Buc-VBM with Oskar eLOTUS domain...................................................... 50

Figure 39: Buc D379L mutation reduces the interaction with Vasa.................................................... 51

Figure 40: Secondary Structure of Vasa-BBM with exposed amino acids. .......................................... 52

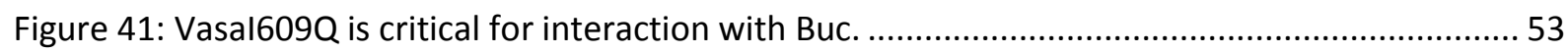

Figure 42: The 1609Q mutation does not reduce stability of Vasa in vivo. ......................................... 53

Figure 43: Vasa helicase activity is not required for germ cell induction. ......................................... 55

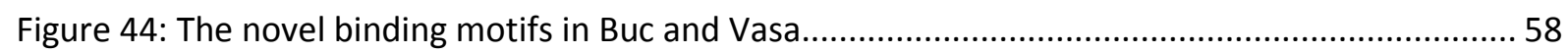

Figure 45: The Buc-VBM is highly conserved among vertebrates................................................... 59

Figure 46: Buc-VBM adopts secondary structure from its disordered state. .................................... 61

Figure 47: Hypothetical model illustrating potential role of Buc, Vasa, RNA germ cell specification. . 65

Figure 48: Hypothetical model illustrating potential role of Buc, Vasa, RNA and Tdrd6a during germ

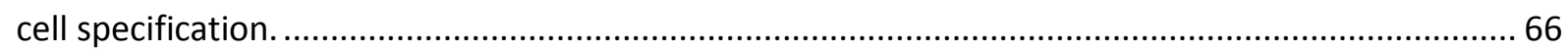

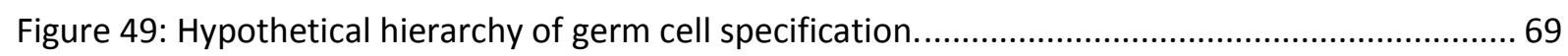

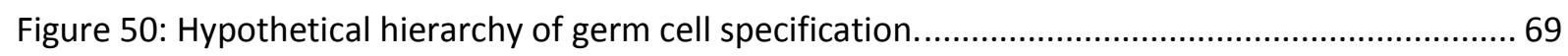




\section{List of Tables}

Table 1. C-score values for predicted zebrafish Vasa homology model ............................................... 36

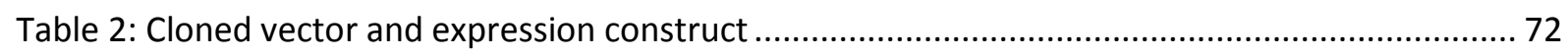

Table 3: Primers used for gateway cloning and In-Fusion cloning ................................................... 82

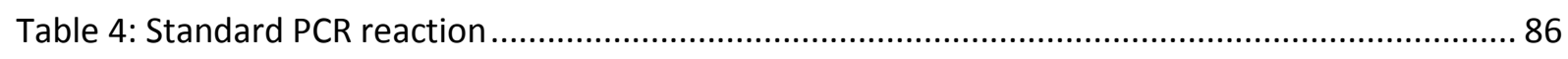

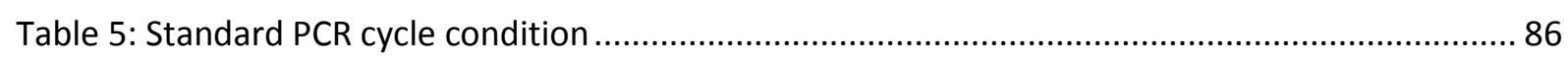

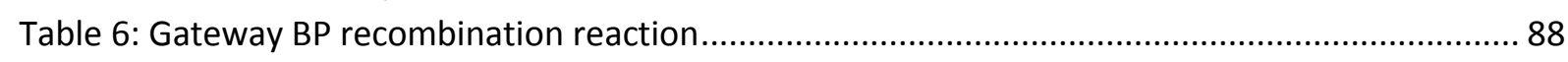

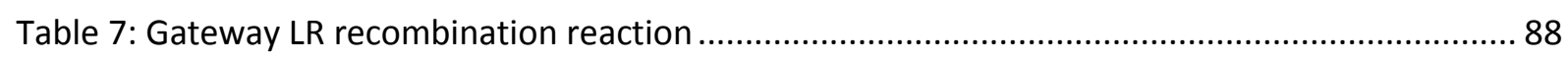

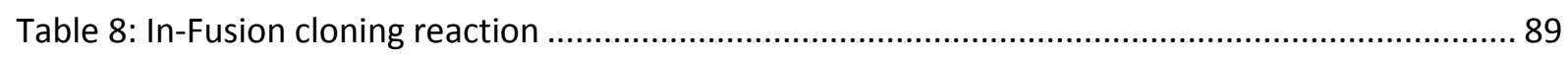

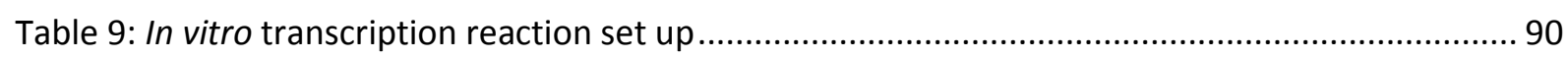




\title{
8. Appendix I-
}

\subsection{Pairwise sequence Alignment of Drosophila Vasa and zebrafish}

\section{Vasa}

\author{
MSA \\ The multiple sequence alignment result as produced by T-coffee. \\ T-COFFEE, Version_11.00.d625267 (2016-01-11 15:25:41 - Revision d625267 - Build 507 \\ Cedric Notredame \\ SCORE $=99$ \\ * BAD AVG GOOD \\ Dm Vasa
zf Vasa \\ cons $\quad \begin{array}{r}9 \\ \text { cons }\end{array}$ \\ Dm Vasa \\ $\mathrm{zf}_{\text {_Vasa }}^{-}$MDDWEEDQSPVVSCSSGFGLGSNGSDGGFKSFYTGGAGNDKSNSEGTEGSSWKMTGDSFRGRGGRGGSRGGR \\ cons \\ Dm_Vasa GGSGGEGGGYQGGNRDVFGRIGGGRGGGAGGYRGGNRDGGGFHGGRREGERDFRGGEGGFRGGQGGSRGGQG \\ $\mathrm{zf}_{\text {-Vasa }}^{-}$GGFSGFKSEIDENGSDGGWNGGESRGKGRGGFRGGFRSGSRDENDENGNDDGWKGGESRGRGRGGFGGGFRG \\ cons
}

Dm Vasa GSRGGQGGFRGGEGGFRGRLYENEDGDERRGRLDREERGGERRGRLDREERGGERGERGDGGFARRRRNEDD $\mathrm{zf}_{-}^{-}$Vasa GFRDGGNEDTGRRGFGRENNENGNDEGEGRGRGRGGFRGGFRDGGGDESGKRGFGRGGFRGRNEEVFSKVTT

cons

Dm_Vasa

INNNNNIVEDVERKREFYIPPEPSNDAIEIFSSGIASGIHFSKYNNIPVKVTGSDVPQPIQHFTSADLRDII $\mathrm{zf}_{-}^{-}$Vasa ADKLDQEGSENAGPKVVYVPPPPPEEESSIFSH - YATGINFDKYDDIL VDVSGSNPPKAIMTFEEAGLCDSL

cons

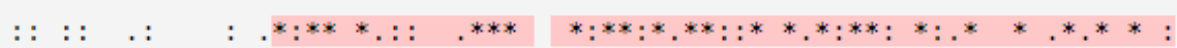

Dm Vasa

IDNVNKSGYKIPTPIOKCSIPVISSGRDLMACAOTGSGKTAAFLLPILSKLLEDPHELELG - . . . . - RPOV $\mathrm{zf}_{-}$Vasa SKNVSKSGYVKPTPVQKHGIPIISAGRDLMACAQTGSGKTAAFLLPILQRFMTDG - - - VAASKFSEIQEPEA

cons

$. * * . * * * * * * * *: * * . * *: * *: * * * * * * * * * * * * * * * * * * * * * * * *,::: *$

**: .

Dm_Vasa VIVSPTRELAIQIFNEARKFAFESYLKIGIVYGG - - TS - - FRHQNECITRGCHVVIATPGRLLDFVDRTFIT $\mathrm{zf}_{\text {-Vasa }}^{-}$IIVAPTRELINQIYLEARKFAYGTCVRPVVVYGGINTGYTIRE - - - -VLKGCNVLCATPGRLHDLIGRGKIG

cons

$: * *: * * * * * \quad * *: * * * * * *:::: \quad: * * * * * . \quad: * . \quad::^{* *}: *: * * * * * * *::$ : $^{*} *$

Dm Vasa FEDTRFVVLDEADRMLDMGFSEDMRRIMTHVTMR - - PEHQTLMFSATFPEE IQRMAGE FLK - NYVFVAIGIV $\mathrm{zf}_{\text {_Vasa }}$ LSKVRYLVLDEADRMLDMGEPEMRKL VASPGMPSKEERQTLMFSATYPEDIQRMAADFLKVDYIFLAVGVV

cons

$: \ldots *:: * * * * * * * * * * * * * .: * *:::: \quad * \quad *: * * * * * * * *: * *: * * * * * .: * * *::^{*}: *: *: *: *$

Dm_Vasa GGACSDVKQTIYEVNKYAKRSKLIEILSE - - QADGTIVFVETKRGADFLASFLSEKEFPTTSIHGDRLQSQR

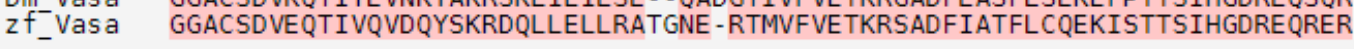

cons

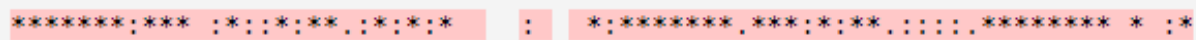

Dm_Vasa EQALRDFKNGSMKVLIATSVASRGLDIKNIKHVINYDMPSKIDDYVHRIGRTGRVGNNGRATSFFDPEKDRA $\mathrm{zf}_{\text {_ }}^{-}$Vasa EKALSDFRLGQCPVLVATSVAARGLDIEQVQHVVNFDMPSSIDEYVHRIGRTGRCGNTGRAVSFFNPESDTP

cons

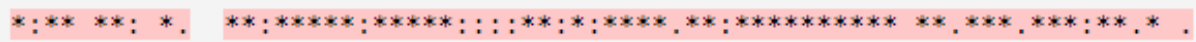

Dm_Vasa IAADLVKILEGSGQTVPDFLRTCGAGGDGGYSNQNFGGVDVRGRGNYVGDATNVEEEEQWD - . . . . . . . . $\mathrm{zf}_{\text {- Vasa }}^{-}$LARSLVKVLSGAQQVVPKWLEEVAFSAHGTTGFNPRGKVFASTDSRKGGSFKSDEPPPSQTSAPSAAAAADD

cons

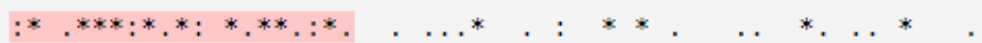

Dm_Vasa ....

$\mathrm{zf}_{\text {-Vasa }}$ EEWE 


\section{Appendix II-}

\subsection{Data file for ATPase assay p55}

\begin{tabular}{|r|r|r|r|}
\hline \multicolumn{1}{|l|}{ Time } & \multicolumn{1}{|l|}{ Vasa } & Buc+Vasa & Buc only \\
\hline 1 & 0 & 0 & 0 \\
\hline 2 & -0.00167 & 0.002667 & 0.003 \\
\hline 3 & -0.002 & 0.002 & 0 \\
\hline 4 & -0.00133 & 0.002667 & 0.001 \\
\hline 5 & -0.00133 & 0.002 & 0.001 \\
\hline 6 & -0.00133 & 0.001667 & 0 \\
\hline 7 & -0.004 & 0.001 & 0 \\
\hline 8 & -0.004 & -0.00133 & 0 \\
\hline 9 & -0.001 & -0.00133 & 0 \\
\hline 10 & -0.00167 & -0.004 & -0.001 \\
\hline 11 & -0.004 & -0.00567 & -0.001 \\
\hline 12 & -0.00333 & -0.00533 & 0 \\
\hline 13 & -0.00367 & -0.006 & 0 \\
\hline 14 & -0.005 & -0.009 & -0.001 \\
\hline 15 & -0.004 & -0.008 & -0.001 \\
\hline 16 & -0.00567 & -0.00867 & -0.001 \\
\hline 17 & -0.00467 & -0.00867 & -0.001 \\
\hline 18 & -0.006 & -0.01067 & -0.001 \\
\hline 19 & -0.006 & -0.00933 & -0.001 \\
\hline 20 & -0.008 & -0.011 & -0.001 \\
\hline 21 & -0.00633 & -0.01133 & -0.003 \\
\hline 22 & -0.00767 & -0.01267 & -0.003 \\
\hline 23 & -0.00567 & -0.011 & -0.001 \\
\hline 24 & -0.00767 & -0.014 & -0.001 \\
\hline 25 & -0.00833 & -0.01367 & -0.001 \\
\hline 26 & -0.00833 & -0.016 & -0.003 \\
\hline 27 & -0.01033 & -0.01633 & -0.001 \\
\hline 28 & -0.00867 & -0.01533 & -0.002 \\
\hline 29 & -0.01067 & -0.017 & -0.001 \\
\hline 30 & -0.01 & -0.017 & -0.003 \\
\hline 31 & -0.01033 & -0.01833 & -0.004 \\
\hline 32 & -0.00967 & -0.01767 & -0.003 \\
\hline 33 & -0.01 & -0.01767 & -0.002 \\
\hline 34 & -0.012 & -0.01867 & -0.004 \\
\hline 35 & -0.01133 & -0.019 & -0.003 \\
\hline 36 & -0.01133 & -0.02067 & -0.005 \\
\hline 37 & -0.01167 & -0.02167 & -0.004 \\
\hline 38 & -0.012 & -0.021 & -0.003 \\
\hline & & & \\
\hline
\end{tabular}




\begin{tabular}{|c|c|c|c|}
\hline 39 & -0.01467 & -0.022 & -0.005 \\
\hline 40 & -0.012 & -0.02033 & -0.005 \\
\hline 41 & -0.01233 & -0.02167 & -0.005 \\
\hline 42 & -0.01433 & -0.02433 & -0.005 \\
\hline 43 & -0.013 & -0.024 & -0.003 \\
\hline 44 & -0.01433 & -0.024 & -0.005 \\
\hline 45 & -0.015 & -0.02567 & -0.005 \\
\hline 46 & -0.01467 & -0.02467 & -0.005 \\
\hline 47 & -0.01767 & -0.02767 & -0.005 \\
\hline 48 & -0.01667 & -0.02733 & -0.005 \\
\hline 49 & -0.01433 & -0.02667 & -0.006 \\
\hline 50 & -0.01733 & -0.02833 & -0.008 \\
\hline 51 & -0.01767 & -0.02967 & -0.005 \\
\hline 52 & -0.01833 & -0.03033 & -0.007 \\
\hline 53 & -0.01867 & -0.03033 & -0.006 \\
\hline 54 & -0.018 & -0.03 & -0.005 \\
\hline 55 & -0.019 & -0.03133 & -0.008 \\
\hline 56 & -0.01867 & -0.033 & -0.005 \\
\hline 57 & -0.01933 & -0.03233 & -0.006 \\
\hline 58 & -0.01967 & -0.03333 & -0.007 \\
\hline 59 & -0.02033 & -0.03267 & -0.007 \\
\hline 60 & -0.019 & -0.034 & -0.007 \\
\hline 61 & -0.01967 & -0.03467 & -0.007 \\
\hline 62 & -0.02133 & -0.03433 & -0.007 \\
\hline 63 & -0.02267 & -0.03633 & -0.007 \\
\hline 64 & -0.02 & -0.03567 & -0.006 \\
\hline 65 & -0.02067 & -0.036 & -0.008 \\
\hline 66 & -0.024 & -0.038 & -0.008 \\
\hline 67 & -0.02167 & -0.037 & -0.008 \\
\hline 68 & -0.02367 & -0.03967 & -0.009 \\
\hline 69 & -0.024 & -0.03967 & -0.008 \\
\hline 70 & -0.02267 & -0.03967 & -0.008 \\
\hline 71 & -0.02333 & -0.04067 & -0.009 \\
\hline 72 & -0.024 & -0.04167 & -0.009 \\
\hline 73 & -0.02433 & -0.041 & -0.008 \\
\hline 74 & -0.02433 & -0.04233 & -0.01 \\
\hline 75 & -0.02567 & -0.042 & -0.009 \\
\hline 76 & -0.025 & -0.04267 & -0.01 \\
\hline 77 & -0.025 & -0.043 & -0.01 \\
\hline 78 & -0.02667 & -0.045 & -0.008 \\
\hline 79 & -0.026 & -0.045 & -0.012 \\
\hline 80 & -0.02633 & -0.04533 & -0.008 \\
\hline 81 & -0.028 & -0.04533 & -0.011 \\
\hline 82 & -0.02733 & -0.047 & -0.01 \\
\hline 83 & -0.02867 & -0.04767 & -0.01 \\
\hline
\end{tabular}




\begin{tabular}{|c|c|c|c|}
\hline 84 & -0.02867 & -0.04767 & -0.01 \\
\hline 85 & -0.029 & -0.04933 & -0.011 \\
\hline 86 & -0.02667 & -0.04733 & -0.011 \\
\hline 87 & -0.02833 & -0.04833 & -0.01 \\
\hline 88 & -0.03067 & -0.05 & -0.011 \\
\hline 89 & -0.031 & -0.05067 & -0.009 \\
\hline 90 & -0.03067 & -0.051 & -0.01 \\
\hline 91 & -0.03133 & -0.052 & -0.008 \\
\hline 92 & -0.03033 & -0.05233 & -0.013 \\
\hline 93 & \begin{tabular}{|r|}
-0.031 \\
\end{tabular} & -0.05233 & -0.009 \\
\hline 94 & -0.031 & -0.05333 & -0.011 \\
\hline 95 & -0.03033 & -0.05267 & -0.012 \\
\hline 96 & -0.03233 & -0.056 & -0.011 \\
\hline 97 & -0.03133 & -0.05467 & -0.008 \\
\hline 98 & -0.033 & -0.056 & -0.012 \\
\hline 99 & -0.03233 & -0.05533 & -0.011 \\
\hline 100 & -0.032 & -0.055 & -0.012 \\
\hline 101 & -0.034 & -0.05567 & -0.012 \\
\hline 102 & -0.034 & -0.058 & -0.011 \\
\hline 103 & -0.035 & -0.059 & -0.011 \\
\hline 104 & -0.03267 & -0.05967 & -0.011 \\
\hline 105 & -0.03667 & -0.06033 & -0.013 \\
\hline 106 & $\begin{array}{l}-0.036 \\
\end{array}$ & -0.06033 & -0.011 \\
\hline 107 & -0.03533 & -0.05933 & -0.012 \\
\hline 108 & -0.03467 & -0.06133 & -0.011 \\
\hline 109 & -0.03733 & -0.06233 & -0.013 \\
\hline 110 & -0.03667 & -0.06267 & -0.012 \\
\hline 111 & \begin{tabular}{|l|}
-0.037 \\
\end{tabular} & -0.06233 & -0.011 \\
\hline 112 & -0.03833 & -0.065 & -0.013 \\
\hline 113 & -0.038 & -0.065 & -0.011 \\
\hline 114 & -0.03833 & -0.06533 & -0.012 \\
\hline 115 & -0.03567 & -0.06433 & -0.013 \\
\hline 116 & -0.03967 & -0.065 & -0.013 \\
\hline 117 & -0.03867 & -0.066 & -0.015 \\
\hline 118 & -0.039 & -0.066 & -0.013 \\
\hline 119 & -0.04067 & -0.06733 & -0.014 \\
\hline 120 & -0.04 & -0.06767 & -0.015 \\
\hline 121 & -0.04033 & -0.068 & -0.013 \\
\hline 122 & -0.04133 & -0.07 & -0.013 \\
\hline 123 & -0.04167 & -0.06967 & -0.014 \\
\hline 124 & -0.04133 & -0.07 & -0.015 \\
\hline 125 & -0.04167 & -0.071 & -0.014 \\
\hline 126 & -0.04133 & -0.072 & -0.013 \\
\hline 127 & -0.04167 & -0.07133 & -0.015 \\
\hline 128 & -0.04267 & -0.07167 & -0.015 \\
\hline
\end{tabular}




\begin{tabular}{|r|r|r|r|}
\hline 129 & -0.04367 & -0.07267 & -0.015 \\
\hline 130 & -0.04267 & -0.07233 & -0.016 \\
\hline 131 & -0.042 & -0.074 & -0.015 \\
\hline 132 & -0.04267 & -0.074 & -0.014 \\
\hline 133 & -0.04433 & -0.07433 & -0.015 \\
\hline 134 & -0.044 & -0.07533 & -0.015 \\
\hline 135 & -0.044 & -0.075 & -0.014 \\
\hline 136 & -0.04333 & -0.07533 & -0.015 \\
\hline 137 & -0.043 & -0.07633 & -0.015 \\
\hline 138 & -0.04533 & -0.07733 & -0.015 \\
\hline 139 & -0.04533 & -0.07767 & -0.016 \\
\hline 140 & -0.04533 & -0.07767 & -0.016 \\
\hline 141 & -0.047 & -0.07867 & -0.015 \\
\hline 142 & -0.04633 & -0.08 & -0.015 \\
\hline 143 & -0.047 & -0.081 & -0.015 \\
\hline 144 & -0.04833 & -0.081 & -0.015 \\
\hline 145 & -0.047 & -0.08067 & -0.016 \\
\hline 146 & -0.048 & -0.082 & -0.015 \\
\hline 147 & -0.047 & -0.08233 & -0.018 \\
\hline 148 & -0.04767 & -0.08267 & -0.016 \\
\hline 149 & -0.04867 & -0.084 & -0.015 \\
\hline 150 & -0.048 & -0.08333 & -0.016 \\
\hline 151 & -0.04733 & -0.08433 & -0.015 \\
\hline 152 & -0.04967 & -0.08533 & -0.015 \\
\hline 153 & -0.04967 & -0.08467 & -0.015 \\
\hline 154 & -0.049 & -0.08433 & -0.017 \\
\hline 155 & -0.05 & -0.08633 & -0.018 \\
\hline 156 & -0.05067 & -0.08833 & -0.017 \\
\hline 157 & -0.052 & -0.088 & -0.019 \\
\hline 158 & -0.05033 & -0.08767 & -0.018 \\
\hline 159 & -0.05167 & -0.089 & -0.017 \\
\hline 160 & -0.05167 & -0.09033 & -0.018 \\
\hline 161 & -0.05133 & -0.08833 & -0.017 \\
\hline 162 & -0.053 & -0.09133 & -0.014 \\
\hline 163 & -0.05433 & -0.09133 & -0.017 \\
\hline 164 & -0.052 & -0.09167 & -0.018 \\
\hline 165 & -0.05267 & -0.092 & -0.016 \\
\hline 166 & -0.054 & -0.09233 & -0.017 \\
\hline 167 & -0.05367 & -0.093 & -0.016 \\
\hline 168 & -0.055 & -0.094 & -0.018 \\
\hline 169 & -0.05633 & -0.09333 & -0.017 \\
\hline 170 & -0.056 & -0.095 & -0.017 \\
\hline 171 & -0.05567 & -0.09467 & -0.018 \\
\hline 173 & -0.05633 & -0.097 & -0.017 \\
\hline & & & \\
\hline
\end{tabular}




\begin{tabular}{|c|c|c|c|}
\hline 174 & -0.056 & -0.09633 & -0.018 \\
\hline 175 & -0.05767 & -0.098 & -0.018 \\
\hline 176 & -0.057 & -0.097 & -0.019 \\
\hline 177 & -0.05733 & -0.09833 & -0.018 \\
\hline 178 & -0.05833 & -0.09767 & -0.018 \\
\hline 179 & -0.05633 & -0.09733 & -0.019 \\
\hline 180 & -0.057 & -0.099 & -0.019 \\
\hline 181 & -0.05767 & -0.09967 & -0.018 \\
\hline 182 & -0.05933 & -0.10167 & -0.019 \\
\hline 183 & -0.057 & -0.10033 & -0.017 \\
\hline 184 & -0.058 & -0.10167 & -0.018 \\
\hline 185 & -0.05933 & -0.102 & -0.019 \\
\hline 186 & -0.061 & -0.10333 & -0.019 \\
\hline 187 & -0.05933 & -0.103 & -0.019 \\
\hline 188 & -0.05967 & -0.10333 & -0.019 \\
\hline 189 & -0.061 & -0.10533 & -0.018 \\
\hline 190 & -0.06233 & -0.10467 & -0.018 \\
\hline 191 & -0.061 & -0.10533 & -0.019 \\
\hline 192 & -0.062 & -0.10633 & -0.018 \\
\hline 193 & -0.062 & -0.107 & -0.019 \\
\hline 194 & -0.05967 & -0.10633 & -0.02 \\
\hline 195 & -0.06367 & -0.10767 & -0.019 \\
\hline 196 & -0.064 & -0.10767 & -0.019 \\
\hline 197 & -0.064 & -0.10867 & -0.02 \\
\hline 198 & -0.06367 & -0.10967 & -0.019 \\
\hline 199 & -0.066 & -0.11133 & -0.018 \\
\hline 200 & -0.065 & -0.11167 & -0.02 \\
\hline 201 & -0.06533 & -0.11067 & -0.019 \\
\hline 202 & -0.06567 & -0.112 & -0.02 \\
\hline 203 & -0.06567 & -0.11167 & -0.019 \\
\hline 204 & -0.06633 & -0.11233 & -0.02 \\
\hline 205 & -0.06433 & -0.11167 & -0.02 \\
\hline 206 & -0.06633 & -0.114 & -0.02 \\
\hline 207 & -0.06733 & -0.11333 & -0.02 \\
\hline 208 & -0.06733 & -0.11533 & -0.02 \\
\hline 209 & -0.06767 & -0.11533 & -0.021 \\
\hline 210 & -0.068 & -0.11467 & -0.021 \\
\hline 211 & -0.06967 & -0.11667 & -0.02 \\
\hline 212 & -0.07033 & -0.11633 & -0.021 \\
\hline 213 & -0.06733 & -0.11733 & -0.02 \\
\hline 214 & -0.06767 & -0.117 & -0.021 \\
\hline 215 & -0.07067 & -0.11767 & -0.021 \\
\hline 216 & -0.071 & -0.119 & -0.021 \\
\hline 217 & -0.07 & -0.11733 & -0.022 \\
\hline 218 & -0.07033 & -0.11933 & -0.021 \\
\hline
\end{tabular}




\begin{tabular}{|c|c|c|c|}
\hline 219 & -0.069 & -0.11933 & -0.021 \\
\hline 220 & -0.06867 & -0.12 & -0.022 \\
\hline 221 & -0.07333 & -0.12233 & -0.022 \\
\hline 222 & -0.07133 & -0.12167 & -0.022 \\
\hline 223 & -0.07233 & -0.122 & -0.021 \\
\hline 224 & -0.07333 & -0.12267 & -0.022 \\
\hline 225 & -0.072 & -0.12333 & -0.022 \\
\hline 226 & -0.07333 & -0.12367 & -0.021 \\
\hline 227 & -0.07267 & -0.12333 & -0.022 \\
\hline 228 & -0.07233 & -0.124 & -0.021 \\
\hline 229 & -0.073 & -0.125 & -0.022 \\
\hline 230 & -0.07467 & -0.12567 & -0.022 \\
\hline 231 & -0.075 & -0.12567 & -0.024 \\
\hline 232 & -0.075 & -0.12633 & -0.022 \\
\hline 233 & -0.07433 & -0.12767 & -0.023 \\
\hline 234 & -0.076 & -0.12767 & -0.023 \\
\hline 235 & -0.075 & -0.12733 & -0.02 \\
\hline 236 & -0.07567 & -0.12767 & -0.022 \\
\hline 237 & -0.07633 & -0.129 & -0.022 \\
\hline 238 & -0.076 & -0.13033 & -0.024 \\
\hline 239 & -0.07733 & -0.13067 & -0.021 \\
\hline 240 & -0.07833 & -0.13067 & -0.024 \\
\hline 241 & -0.07967 & -0.13267 & -0.023 \\
\hline 242 & -0.07833 & -0.13167 & -0.023 \\
\hline 243 & -0.07867 & -0.13333 & -0.022 \\
\hline 244 & -0.07833 & -0.13367 & -0.025 \\
\hline 245 & -0.079 & -0.13333 & -0.023 \\
\hline 246 & -0.07767 & -0.13367 & -0.024 \\
\hline 247 & -0.07933 & -0.135 & -0.025 \\
\hline 248 & -0.08033 & -0.13533 & -0.025 \\
\hline 249 & -0.082 & -0.13567 & -0.025 \\
\hline 250 & -0.081 & -0.137 & -0.022 \\
\hline 251 & -0.08133 & -0.13733 & -0.026 \\
\hline 252 & -0.08167 & -0.13733 & -0.024 \\
\hline 253 & -0.08167 & -0.138 & -0.025 \\
\hline 254 & -0.081 & -0.137 & -0.026 \\
\hline 255 & -0.08367 & -0.139 & -0.025 \\
\hline 256 & -0.082 & -0.13967 & -0.025 \\
\hline 257 & -0.08267 & -0.14 & -0.023 \\
\hline 258 & -0.08167 & -0.13867 & -0.025 \\
\hline 259 & -0.084 & -0.14033 & -0.024 \\
\hline 260 & -0.08533 & -0.14167 & -0.027 \\
\hline 261 & -0.08367 & -0.141 & -0.025 \\
\hline 262 & -0.08567 & -0.14167 & -0.025 \\
\hline 263 & -0.08667 & -0.144 & -0.026 \\
\hline
\end{tabular}




\begin{tabular}{|c|c|c|c|}
\hline 264 & -0.08567 & -0.14367 & -0.024 \\
\hline 265 & -0.08567 & -0.14433 & -0.026 \\
\hline 266 & -0.086 & -0.14433 & -0.025 \\
\hline 267 & -0.086 & -0.145 & -0.025 \\
\hline 268 & -0.086 & -0.144 & -0.026 \\
\hline 269 & -0.08633 & -0.14533 & -0.025 \\
\hline 270 & -0.088 & -0.14733 & -0.025 \\
\hline 271 & -0.08833 & -0.147 & -0.025 \\
\hline 272 & -0.08833 & -0.14733 & -0.025 \\
\hline 273 & -0.08833 & -0.148 & -0.025 \\
\hline 274 & -0.09033 & -0.149 & -0.025 \\
\hline 275 & -0.08867 & -0.14833 & -0.027 \\
\hline 276 & -0.08967 & -0.14967 & -0.025 \\
\hline 277 & -0.091 & -0.15 & -0.026 \\
\hline 278 & -0.08967 & -0.15067 & -0.026 \\
\hline 279 & -0.09133 & -0.15133 & -0.025 \\
\hline 280 & -0.09133 & -0.15167 & -0.026 \\
\hline 281 & -0.093 & -0.15267 & -0.026 \\
\hline 282 & -0.091 & -0.152 & -0.028 \\
\hline 283 & -0.09367 & -0.154 & -0.027 \\
\hline 284 & -0.093 & -0.15367 & -0.028 \\
\hline 285 & -0.094 & -0.15433 & -0.027 \\
\hline 286 & -0.092 & -0.15433 & -0.025 \\
\hline 287 & -0.09433 & -0.15567 & -0.027 \\
\hline 288 & -0.094 & -0.15633 & -0.026 \\
\hline 289 & -0.09433 & -0.157 & -0.027 \\
\hline 290 & -0.09367 & -0.156 & -0.027 \\
\hline 291 & -0.093 & -0.15667 & -0.027 \\
\hline 292 & -0.09633 & -0.15867 & -0.025 \\
\hline 293 & -0.09633 & -0.15933 & -0.026 \\
\hline 294 & -0.098 & -0.15967 & -0.027 \\
\hline 295 & -0.09567 & -0.15933 & -0.026 \\
\hline 296 & -0.097 & -0.15933 & -0.026 \\
\hline 297 & -0.09633 & -0.16033 & -0.029 \\
\hline 298 & -0.09633 & -0.16033 & -0.027 \\
\hline 299 & -0.09867 & -0.162 & -0.028 \\
\hline 300 & -0.09567 & -0.162 & -0.027 \\
\hline 301 & -0.09833 & -0.16367 & -0.028 \\
\hline 302 & -0.098 & -0.16333 & -0.026 \\
\hline 303 & -0.099 & -0.16267 & -0.027 \\
\hline 304 & -0.098 & -0.16333 & -0.029 \\
\hline 305 & -0.10133 & -0.16367 & -0.029 \\
\hline 306 & -0.098 & -0.164 & -0.027 \\
\hline 307 & -0.10133 & -0.16467 & -0.027 \\
\hline 308 & -0.10067 & -0.167 & -0.028 \\
\hline
\end{tabular}




\begin{tabular}{|r|r|r|r|}
\hline 309 & -0.1 & -0.16633 & -0.029 \\
\hline 310 & -0.10033 & -0.16633 & -0.027 \\
\hline 311 & -0.101 & -0.16767 & -0.03 \\
\hline 312 & -0.101 & -0.168 & -0.029 \\
\hline
\end{tabular}




\section{Appendix III}

\subsection{Data for ATPase assay p55}

\begin{tabular}{|c|c|c|c|c|c|}
\hline Time (min) & Buc+Vasa+RNA & Buc+Vasa & Vasa+RNA & Buc & Vasa \\
\hline 1 & 0 & 0 & 0 & 0 & 0 \\
\hline 2 & $3.33 \mathrm{E}+01$ & $1.00 \mathrm{E}-03$ & $-6.67 E+01$ & 0 & $6.67 E+01$ \\
\hline 3 & $-0,00133$ & 0,00133 & $-0,00133$ & 0 & $3.33 E+01$ \\
\hline 4 & $-3.33 E+01$ & $1.00 \mathrm{E}-03$ & $-3.33 E+01$ & 0 & $3.33 \mathrm{E}+01$ \\
\hline 5 & $-0,00233$ & $6.67 E+01$ & $-6.67 E+01$ & 0 & $3.33 E+01$ \\
\hline 6 & $-0,00267$ & $-0,002$ & $-0,00133$ & 3.33E+01 & $6.67 \mathrm{E}+01$ \\
\hline 7 & $-0,00333$ & $-0,00167$ & $-0,00233$ & $6.67 \mathrm{E}+01$ & $3.33 \mathrm{E}+01$ \\
\hline 8 & $-0,00433$ & $-0,00167$ & $-0,002$ & $\begin{array}{r}-1.00 \mathrm{E}- \\
03\end{array}$ & $-0,00167$ \\
\hline 9 & $-0,005$ & $-0,00267$ & $-0,00133$ & $\begin{array}{r}-1.00 \mathrm{E}- \\
03 \\
\end{array}$ & $\begin{array}{r}-1.00 \mathrm{E}- \\
03 \\
\end{array}$ \\
\hline 10 & $-0,00533$ & $-0,00267$ & $-0,002$ & $\begin{array}{r}- \\
3.33 \mathrm{E}+01 \\
\end{array}$ & $-0,00167$ \\
\hline 11 & $-0,007$ & $-0,004$ & $-0,003$ & $-0,00133$ & $-0,00167$ \\
\hline 12 & $-0,00833$ & $-0,005$ & $-0,00333$ & $-0,00133$ & $-0,00267$ \\
\hline 13 & $-0,00733$ & $-0,00467$ & $-0,00167$ & $\begin{array}{r}- \\
6.67 \mathrm{E}+01\end{array}$ & $\begin{array}{r}-1.00 \mathrm{E}- \\
03 \\
\end{array}$ \\
\hline 14 & $-0,00833$ & $-0,005$ & $-0,002$ & $-0,00133$ & $-0,00233$ \\
\hline 15 & $-0,00867$ & $-0,00567$ & $-0,00433$ & $-0,00167$ & $-0,00267$ \\
\hline 16 & $-0,01167$ & $-0,00733$ & $-0,00467$ & $-0,00167$ & $-0,00367$ \\
\hline 17 & $-0,01133$ & $-0,00733$ & $-0,00433$ & $-0,00167$ & $-0,00267$ \\
\hline 18 & $-0,01233$ & $-0,00833$ & $-0,00433$ & $-0,00233$ & $-0,00333$ \\
\hline 19 & $-0,012$ & $-0,008$ & $-0,005$ & $-0,00133$ & $-0,003$ \\
\hline 20 & $-0,01233$ & $-0,00833$ & $-0,004$ & $3.33 E+01$ & $-0,00267$ \\
\hline 21 & $-0,01433$ & $-0,00933$ & $-0,00567$ & $-0,00267$ & $-0,00467$ \\
\hline 22 & $-0,013$ & $-0,00933$ & $-0,00467$ & $\begin{array}{r}-1.00 \mathrm{E}- \\
03 \\
\end{array}$ & $-0,00333$ \\
\hline 23 & $-0,016$ & $-0,01033$ & $-0,00533$ & $-0,00267$ & $-0,00433$ \\
\hline 24 & $-0,016$ & $-0,01267$ & $-0,00633$ & $-0,00133$ & $-0,00467$ \\
\hline 25 & $-0,01667$ & $-0,01267$ & $-0,00667$ & $-0,00333$ & $-0,00467$ \\
\hline 26 & $-0,01667$ & $-0,011$ & $-0,00567$ & $-0,00233$ & $-0,004$ \\
\hline 27 & $-0,01633$ & $-0,012$ & $-0,006$ & $-0,00233$ & $-0,00367$ \\
\hline 28 & $-0,01733$ & $-0,013$ & $-0,00733$ & $-0,00167$ & $-0,00467$ \\
\hline 29 & $-0,018$ & $-0,014$ & $-0,00633$ & $-0,00233$ & $-0,005$ \\
\hline 30 & $-0,01933$ & $-0,01467$ & $-0,00667$ & $-0,003$ & $-0,005$ \\
\hline
\end{tabular}




\begin{tabular}{|c|c|c|c|c|c|}
\hline 31 & $-0,02$ & $-0,01433$ & $-0,008$ & $-0,00233$ & $-0,00533$ \\
\hline 32 & $-0,02033$ & $-0,016$ & $-0,008$ & $-0,00267$ & $-0,00533$ \\
\hline 33 & $-0,02067$ & $-0,01533$ & $-0,008$ & $-0,00267$ & $-0,00533$ \\
\hline 34 & $-0,021$ & $-0,016$ & $-0,00733$ & $-0,00233$ & $-0,00567$ \\
\hline 35 & $-0,02267$ & $-0,01833$ & $-0,00967$ & $-0,00367$ & $-0,00667$ \\
\hline 36 & $-0,02167$ & $-0,01733$ & $-0,00867$ & $-0,00233$ & $-0,006$ \\
\hline 37 & $-0,023$ & $-0,01833$ & $-0,009$ & $-0,00333$ & $-0,00767$ \\
\hline 38 & $-0,02433$ & $-0,018$ & $-0,00967$ & $-0,00267$ & $-0,00633$ \\
\hline 39 & $-0,02567$ & $-0,02033$ & $-0,01067$ & $-0,00433$ & $-0,00767$ \\
\hline 40 & $-0,02533$ & $-0,01967$ & $-0,01$ & $-0,003$ & $-0,00667$ \\
\hline 41 & $-0,02633$ & $-0,02067$ & $-0,01067$ & $-0,00367$ & $-0,007$ \\
\hline 42 & $-0,02567$ & $-0,02$ & $-0,01033$ & $-0,003$ & $-0,007$ \\
\hline 43 & $-0,027$ & $-0,02067$ & $-0,01067$ & $-0,00367$ & $-0,00833$ \\
\hline 44 & $-0,026$ & $-0,02067$ & $-0,01$ & $-0,003$ & $-0,00667$ \\
\hline 45 & $-0,028$ & $-0,02167$ & $-0,011$ & $-0,003$ & $-0,00767$ \\
\hline 46 & $-0,028$ & $-0,02267$ & $-0,012$ & $-0,00233$ & $-0,008$ \\
\hline 47 & $-0,02833$ & $-0,023$ & $-0,011$ & $-0,00333$ & $-0,008$ \\
\hline 48 & $-0,03067$ & $-0,024$ & $-0,01233$ & $-0,00367$ & $-0,009$ \\
\hline 49 & $-0,03033$ & $-0,02333$ & $-0,013$ & $-0,003$ & $-0,00867$ \\
\hline 50 & $-0,03$ & $-0,02367$ & $-0,01233$ & $-0,00333$ & $-0,00833$ \\
\hline 51 & $-0,03167$ & $-0,02533$ & $-0,013$ & $-0,003$ & $-0,009$ \\
\hline 52 & $-0,03267$ & $-0,02567$ & $-0,014$ & $-0,003$ & $-0,00933$ \\
\hline 53 & $-0,034$ & $-0,02733$ & $-0,015$ & $-0,006$ & $-0,01067$ \\
\hline 54 & $-0,03333$ & $-0,02667$ & $-0,01467$ & $-0,00467$ & $-0,01$ \\
\hline 55 & $-0,03467$ & $-0,02733$ & $-0,01433$ & $-0,00367$ & $-0,01067$ \\
\hline 56 & $-0,035$ & $-0,02833$ & $-0,015$ & $-0,00533$ & $-0,00933$ \\
\hline 57 & $-0,03533$ & $-0,029$ & $-0,01533$ & $-0,00567$ & $-0,01067$ \\
\hline 58 & $-0,036$ & $-0,02833$ & $-0,015$ & $-0,004$ & $-0,01067$ \\
\hline 59 & $-0,03667$ & $-0,02967$ & $-0,016$ & $-0,00467$ & $-0,011$ \\
\hline 60 & $-0,037$ & $-0,03$ & $-0,01533$ & $-0,00467$ & $-0,011$ \\
\hline 61 & $-0,03633$ & $-0,02867$ & $-0,01567$ & $-0,00433$ & $-0,01033$ \\
\hline 62 & $-0,03733$ & $-0,03$ & $-0,015$ & $-0,004$ & $-0,01033$ \\
\hline 63 & $-0,03833$ & $-0,03067$ & $-0,016$ & $-0,00367$ & $-0,01067$ \\
\hline 64 & $-0,04033$ & $-0,033$ & $-0,018$ & $-0,005$ & $-0,013$ \\
\hline 65 & $-0,04$ & $-0,033$ & $-0,017$ & $-0,005$ & $-0,01267$ \\
\hline 66 & $-0,04133$ & $-0,033$ & $-0,01867$ & $-0,005$ & $-0,013$ \\
\hline 67 & $-0,04067$ & $-0,03267$ & $-0,01733$ & $-0,00367$ & $-0,01167$ \\
\hline 68 & $-0,04167$ & $-0,03333$ & $-0,019$ & $-0,005$ & $-0,01167$ \\
\hline 69 & $-0,042$ & $-0,03367$ & $-0,01767$ & $-0,004$ & $-0,01233$ \\
\hline 70 & $-0,04333$ & $-0,03467$ & $-0,01833$ & $-0,005$ & $-0,013$ \\
\hline 71 & $-0,04333$ & $-0,03367$ & $-0,019$ & $-0,00533$ & $-0,013$ \\
\hline 72 & $-0,04433$ & $-0,03633$ & $-0,019$ & $-0,00567$ & $-0,015$ \\
\hline 73 & $-0,04433$ & $-0,03567$ & $-0,01933$ & $-0,00567$ & $-0,013$ \\
\hline 74 & $-0,044$ & $-0,03533$ & $-0,01933$ & $-0,00433$ & $-0,01233$ \\
\hline 75 & $-0,04567$ & $-0,036$ & $-0,01933$ & $-0,00533$ & $-0,013$ \\
\hline
\end{tabular}




\begin{tabular}{|c|c|c|c|c|c|}
\hline 76 & $-0,04633$ & $-0,03733$ & $-0,021$ & $-0,00533$ & $-0,014$ \\
\hline 77 & $-0,04633$ & $-0,037$ & $-0,02033$ & $-0,00533$ & $-0,01533$ \\
\hline 78 & $-0,04567$ & $-0,03767$ & $-0,02067$ & $-0,00433$ & $-0,014$ \\
\hline 79 & $-0,047$ & $-0,03867$ & $-0,02033$ & $-0,00533$ & $-0,01433$ \\
\hline 80 & $-0,04833$ & $-0,03933$ & $-0,022$ & $-0,00533$ & $-0,01533$ \\
\hline 81 & $-0,049$ & $-0,04$ & $-0,02167$ & $-0,00567$ & $-0,01567$ \\
\hline 82 & $-0,04933$ & $-0,03967$ & $-0,022$ & $-0,006$ & $-0,01567$ \\
\hline 83 & $-0,05033$ & $-0,04033$ & $-0,02167$ & $-0,00633$ & $-0,016$ \\
\hline 84 & $-0,05033$ & $-0,04067$ & $-0,02167$ & $-0,00533$ & $-0,015$ \\
\hline 85 & $-0,05067$ & $-0,041$ & $-0,02267$ & $-0,00533$ & $-0,01533$ \\
\hline 86 & $-0,052$ & $-0,04167$ & $-0,024$ & $-0,00567$ & $-0,01567$ \\
\hline 87 & \begin{tabular}{|l|}
$-0,052$ \\
\end{tabular} & $-0,04233$ & $-0,02333$ & $-0,006$ & $-0,01533$ \\
\hline 88 & $-0,05233$ & $-0,04333$ & $-0,02333$ & $-0,006$ & $-0,01667$ \\
\hline 89 & $-0,05333$ & $-0,04167$ & $-0,023$ & $-0,00533$ & $-0,016$ \\
\hline 90 & $-0,054$ & $-0,04333$ & $-0,02333$ & $-0,006$ & $-0,01633$ \\
\hline 91 & $-0,054$ & $-0,04433$ & $-0,02467$ & $-0,00533$ & $-0,01733$ \\
\hline 92 & $-0,05367$ & $-0,04433$ & $-0,02333$ & $-0,00533$ & $-0,01567$ \\
\hline 93 & $-0,05433$ & $-0,04367$ & $-0,024$ & $-0,00533$ & $-0,016$ \\
\hline 94 & $-0,056$ & $-0,045$ & $-0,025$ & $-0,00567$ & $-0,01733$ \\
\hline 95 & $-0,056$ & $-0,046$ & $-0,02467$ & $-0,00567$ & $-0,01733$ \\
\hline 96 & $-0,05533$ & $-0,04567$ & $-0,024$ & $-0,006$ & $-0,01633$ \\
\hline 97 & $-0,05767$ & $-0,04667$ & $-0,026$ & $-0,00633$ & $-0,01767$ \\
\hline 98 & $-0,058$ & $-0,04733$ & $-0,02633$ & $-0,007$ & $-0,01867$ \\
\hline 99 & \begin{tabular}{|l|}
$-0,059$ \\
\end{tabular} & $-0,04767$ & $-0,027$ & $-0,007$ & $-0,01833$ \\
\hline 100 & $-0,05933$ & $-0,04733$ & $-0,02667$ & $-0,006$ & $-0,01867$ \\
\hline 101 & $-0,059$ & $-0,04867$ & $-0,02533$ & $-0,00633$ & $-0,018$ \\
\hline 102 & $-0,06$ & $-0,04867$ & $-0,02767$ & $-0,006$ & $-0,01867$ \\
\hline 103 & $-0,05967$ & $-0,04833$ & $-0,02767$ & $-0,007$ & $-0,01933$ \\
\hline 104 & $-0,061$ & $-0,04967$ & $-0,026$ & $-0,006$ & $-0,01767$ \\
\hline 105 & $-0,06233$ & $-0,05$ & $-0,027$ & $-0,00633$ & $-0,01867$ \\
\hline 106 & $-0,06167$ & $-0,05$ & $-0,02733$ & $-0,00633$ & $-0,018$ \\
\hline 107 & $-0,06433$ & $-0,05233$ & $-0,02833$ & $-0,007$ & $-0,02$ \\
\hline 108 & $-0,063$ & $-0,05133$ & $-0,02733$ & $-0,00633$ & $-0,01967$ \\
\hline 109 & $-0,064$ & $-0,05233$ & $-0,02967$ & $-0,008$ & $-0,02067$ \\
\hline 110 & $-0,064$ & $-0,05267$ & $-0,02967$ & $-0,00733$ & $-0,02$ \\
\hline 111 & $-0,06467$ & $-0,05267$ & $-0,029$ & $-0,00667$ & $-0,02$ \\
\hline 112 & $-0,06567$ & $-0,05167$ & $-0,029$ & $-0,00667$ & $-0,01967$ \\
\hline 113 & $-0,065$ & $-0,05267$ & $-0,02867$ & $-0,00667$ & $-0,01933$ \\
\hline 114 & $-0,06567$ & $-0,05367$ & $-0,02933$ & $-0,007$ & $-0,02$ \\
\hline 115 & $-0,067$ & $-0,05433$ & $-0,029$ & $-0,006$ & $-0,02$ \\
\hline 116 & $-0,06833$ & $-0,05467$ & $-0,03$ & $-0,00733$ & $-0,02133$ \\
\hline 117 & $-0,06667$ & $-0,05467$ & $-0,031$ & $-0,007$ & $-0,021$ \\
\hline 118 & $-0,06833$ & $-0,05533$ & $-0,02933$ & $-0,00733$ & $-0,021$ \\
\hline 119 & $-0,06867$ & $-0,05567$ & $-0,03033$ & $-0,007$ & $-0,02167$ \\
\hline 120 & $-0,06867$ & $-0,05567$ & $-0,03067$ & $-0,006$ & $-0,02033$ \\
\hline
\end{tabular}




\begin{tabular}{|c|c|c|c|c|c|}
\hline 121 & $-0,06933$ & $-0,05633$ & $-0,03167$ & $-0,007$ & $-0,021$ \\
\hline 122 & $-0,06967$ & $-0,05633$ & $-0,032$ & $-0,007$ & $-0,02067$ \\
\hline 123 & $-0,06933$ & $-0,05667$ & $-0,03133$ & $-0,007$ & $-0,021$ \\
\hline 124 & $-0,07067$ & $-0,05767$ & $-0,031$ & $-0,00667$ & $-0,021$ \\
\hline 125 & $-0,07267$ & $-0,05867$ & $-0,033$ & $-0,00733$ & $-0,02233$ \\
\hline 126 & $-0,07133$ & $-0,05833$ & $-0,032$ & $-0,00667$ & $-0,02133$ \\
\hline 127 & $-0,07233$ & $-0,059$ & $-0,032$ & $-0,00733$ & $-0,02167$ \\
\hline 128 & $-0,07267$ & $-0,05967$ & $-0,032$ & $-0,008$ & $-0,02167$ \\
\hline 129 & $-0,074$ & $-0,06067$ & $-0,03467$ & $-0,00767$ & $-0,02333$ \\
\hline 130 & $-0,075$ & $-0,06067$ & $-0,034$ & $-0,008$ & $-0,02267$ \\
\hline 131 & $-0,074$ & $-0,06033$ & $-0,03333$ & $-0,00667$ & $-0,02233$ \\
\hline 132 & $-0,07467$ & $-0,06133$ & $-0,03367$ & $-0,007$ & $-0,02333$ \\
\hline 133 & $-0,07667$ & $-0,063$ & $-0,03533$ & $-0,00767$ & $-0,02367$ \\
\hline 134 & $-0,077$ & $-0,06233$ & $-0,03467$ & $-0,008$ & $-0,02367$ \\
\hline 135 & $-0,07633$ & $-0,06267$ & $-0,035$ & $-0,00733$ & $-0,02333$ \\
\hline 136 & $-0,077$ & $-0,06233$ & $-0,035$ & $-0,00767$ & $-0,024$ \\
\hline 137 & $-0,07733$ & $-0,06167$ & $-0,03433$ & $-0,007$ & $-0,02433$ \\
\hline 138 & $-0,078$ & $-0,063$ & $-0,035$ & $-0,00733$ & $-0,025$ \\
\hline 139 & $-0,07933$ & $-0,065$ & $-0,03733$ & $-0,009$ & $-0,02567$ \\
\hline 140 & $-0,078$ & $-0,06367$ & $-0,03567$ & $-0,00733$ & $-0,02367$ \\
\hline 141 & $-0,079$ & $-0,06433$ & $-0,03667$ & $-0,00633$ & $-0,024$ \\
\hline 142 & $-0,08$ & $-0,06533$ & $-0,037$ & $-0,008$ & $-0,02467$ \\
\hline 143 & $-0,08$ & $-0,06533$ & $-0,03633$ & $-0,00833$ & $-0,02433$ \\
\hline 144 & $-0,082$ & $-0,066$ & $-0,03733$ & $-0,00833$ & $-0,02533$ \\
\hline 145 & $-0,08233$ & $-0,06633$ & $-0,03767$ & $-0,00867$ & $-0,02567$ \\
\hline 146 & $-0,08267$ & $-0,06633$ & $-0,03767$ & $-0,00833$ & $-0,02567$ \\
\hline 147 & $-0,083$ & $-0,06733$ & $-0,03867$ & $-0,00833$ & $-0,02633$ \\
\hline 148 & $-0,083$ & $-0,06767$ & $-0,03833$ & $-0,008$ & $-0,02567$ \\
\hline 149 & $-0,08467$ & $-0,06833$ & $-0,04$ & $-0,00933$ & $-0,02667$ \\
\hline 150 & $-0,085$ & $-0,06933$ & $-0,03933$ & $-0,01033$ & $-0,027$ \\
\hline 151 & $-0,084$ & $-0,067$ & $-0,038$ & $-0,008$ & $-0,026$ \\
\hline 152 & $-0,08567$ & $-0,06967$ & $-0,03933$ & $-0,00933$ & $-0,027$ \\
\hline 153 & $-0,08533$ & $-0,06967$ & $-0,04$ & $-0,00767$ & $-0,027$ \\
\hline 154 & $-0,08633$ & $-0,07$ & $-0,04033$ & $-0,00867$ & $-0,02767$ \\
\hline 155 & $-0,08767$ & $-0,07067$ & $-0,04067$ & $-0,009$ & $-0,028$ \\
\hline 156 & $-0,08733$ & $-0,071$ & $-0,041$ & $-0,009$ & $-0,02767$ \\
\hline 157 & $-0,088$ & \begin{tabular}{|l|}
$-0,07$ \\
\end{tabular} & $-0,04033$ & $-0,008$ & $-0,02667$ \\
\hline 158 & $-0,08833$ & $-0,07167$ & $-0,042$ & $-0,00867$ & $-0,028$ \\
\hline 159 & $-0,08867$ & $-0,07133$ & $-0,04133$ & $-0,00833$ & $-0,02733$ \\
\hline 160 & $-0,08867$ & $-0,07167$ & $-0,04167$ & $-0,008$ & $-0,02833$ \\
\hline 161 & $-0,08867$ & $-0,07133$ & $-0,041$ & $-0,00833$ & $-0,027$ \\
\hline 162 & $-0,09033$ & $-0,07333$ & $-0,04333$ & $-0,00967$ & $-0,029$ \\
\hline 163 & $-0,09$ & $-0,07333$ & $-0,042$ & $-0,00833$ & $-0,02867$ \\
\hline 164 & $-0,091$ & $-0,07333$ & $-0,04233$ & $-0,00967$ & $-0,02933$ \\
\hline 165 & $-0,092$ & $-0,07433$ & $-0,044$ & $-0,01$ & $-0,029$ \\
\hline
\end{tabular}




\begin{tabular}{|c|c|c|c|c|c|}
\hline 166 & $-0,09133$ & $-0,07433$ & $-0,04267$ & $-0,00933$ & $-0,028$ \\
\hline 167 & $-0,09233$ & $-0,07367$ & $-0,04233$ & $-0,00967$ & $-0,02933$ \\
\hline 168 & $-0,09267$ & $-0,07633$ & $-0,04467$ & $-0,00967$ & $-0,02967$ \\
\hline 169 & $-0,093$ & $-0,076$ & $-0,044$ & $-0,00933$ & $-0,03$ \\
\hline 170 & $-0,094$ & $-0,077$ & $-0,044$ & $-0,01033$ & $-0,02933$ \\
\hline 171 & $-0,09433$ & \begin{tabular}{|l|}
$-0,07733$ \\
\end{tabular} & $-0,044$ & $-0,01067$ & $-0,03067$ \\
\hline 172 & $-0,09467$ & $-0,076$ & $-0,04367$ & $-0,00967$ & $-0,03$ \\
\hline 173 & $-0,09567$ & $-0,07733$ & $-0,04467$ & $-0,00933$ & $-0,03067$ \\
\hline 174 & $-0,095$ & $-0,07767$ & $-0,04533$ & $-0,009$ & $-0,03033$ \\
\hline 175 & $-0,09633$ & $-0,07833$ & $-0,045$ & $-0,00967$ & $-0,031$ \\
\hline 176 & $-0,097$ & $-0,07767$ & $-0,04533$ & $-0,01067$ & $-0,03067$ \\
\hline 177 & $-0,09667$ & $-0,079$ & $-0,04633$ & $-0,01033$ & $-0,03$ \\
\hline 178 & $-0,09767$ & $-0,079$ & $-0,047$ & $-0,00967$ & $-0,03133$ \\
\hline 179 & $-0,09833$ & $-0,08033$ & $-0,04733$ & $-0,01033$ & $-0,031$ \\
\hline 180 & $-0,09767$ & $-0,07967$ & $-0,04633$ & $-0,00967$ & $-0,031$ \\
\hline 181 & $-0,09833$ & $-0,07933$ & $-0,046$ & $-0,009$ & $-0,03067$ \\
\hline 182 & $-0,09967$ & $-0,081$ & $-0,04667$ & $-0,01067$ & $-0,03133$ \\
\hline 183 & $-0,10033$ & $-0,081$ & $-0,04667$ & $-0,01067$ & $-0,03133$ \\
\hline 184 & $-0,09933$ & $-0,08033$ & $-0,04733$ & $-0,01033$ & $-0,03133$ \\
\hline 185 & $-0,101$ & $-0,082$ & $-0,04833$ & $-0,01$ & $-0,03267$ \\
\hline 186 & $-0,10167$ & $-0,08267$ & $-0,05067$ & $-0,011$ & $-0,03367$ \\
\hline 187 & $-0,10233$ & $-0,083$ & $-0,04933$ & $-0,01033$ & $-0,03333$ \\
\hline 188 & $-0,102$ & $-0,08367$ & $-0,04733$ & $-0,01067$ & $-0,033$ \\
\hline 189 & \begin{tabular}{|l|}
$-0,102$ \\
\end{tabular} & $-0,08367$ & $-0,04733$ & $-0,01167$ & $-0,03333$ \\
\hline 190 & $-0,10067$ & $-0,082$ & $-0,046$ & $-0,00867$ & $-0,03067$ \\
\hline 191 & $-0,10133$ & $-0,08267$ & $-0,047$ & $-0,00933$ & $-0,03233$ \\
\hline 192 & $-0,10067$ & $-0,08267$ & $-0,047$ & $-0,00933$ & $-0,032$ \\
\hline 193 & $-0,10167$ & $-0,08367$ & $-0,04667$ & $-0,00833$ & $-0,032$ \\
\hline 194 & $-0,102$ & $-0,083$ & $-0,047$ & $-0,00967$ & $-0,03133$ \\
\hline 195 & $-0,103$ & $-0,08467$ & $-0,048$ & $-0,00933$ & $-0,03167$ \\
\hline 196 & $-0,10367$ & $-0,084$ & $-0,047$ & $-0,00933$ & $-0,03233$ \\
\hline 197 & $-0,104$ & $-0,084$ & $-0,04733$ & $-0,00933$ & $-0,033$ \\
\hline 198 & $-0,105$ & $-0,08667$ & $-0,04967$ & $-0,01033$ & $-0,03333$ \\
\hline 199 & $-0,10567$ & $-0,086$ & $-0,04867$ & $-0,00967$ & $-0,03267$ \\
\hline 200 & $-0,106$ & $-0,086$ & $-0,04967$ & $-0,01033$ & $-0,03367$ \\
\hline 201 & $-0,10533$ & $-0,08633$ & $-0,049$ & $-0,00933$ & $-0,03333$ \\
\hline 202 & $-0,10633$ & $-0,08667$ & $-0,04933$ & $-0,00967$ & $-0,03333$ \\
\hline 203 & $-0,106$ & $-0,08667$ & $-0,04933$ & $-0,00967$ & $-0,03333$ \\
\hline 204 & $-0,10767$ & $-0,08733$ & $-0,051$ & $-0,01033$ & $-0,034$ \\
\hline 205 & $-0,10767$ & $-0,08833$ & $-0,05033$ & $-0,01067$ & $-0,03467$ \\
\hline 206 & $-0,10733$ & $-0,08733$ & $-0,05067$ & $-0,01067$ & $-0,03467$ \\
\hline 207 & $-0,10867$ & $-0,08833$ & $-0,05133$ & $-0,01033$ & $-0,035$ \\
\hline 208 & $-0,10967$ & $-0,09$ & $-0,05167$ & $-0,011$ & $-0,03533$ \\
\hline 209 & $-0,109$ & $-0,089$ & $-0,05133$ & $-0,011$ & $-0,03533$ \\
\hline 210 & $-0,109$ & $-0,089$ & $-0,05133$ & $-0,01$ & $-0,03433$ \\
\hline
\end{tabular}




\begin{tabular}{|c|c|c|c|c|c|}
\hline 211 & $-0,11033$ & $-0,08933$ & $-0,052$ & $-0,01133$ & $-0,03533$ \\
\hline 212 & $-0,10967$ & $-0,08967$ & $-0,051$ & $-0,00967$ & $-0,034$ \\
\hline 213 & $-0,111$ & $-0,091$ & $-0,05233$ & $-0,00967$ & $-0,03467$ \\
\hline 214 & $-0,11233$ & $-0,09167$ & $-0,05367$ & $-0,011$ & $-0,03633$ \\
\hline 215 & $-0,112$ & $-0,091$ & $-0,05267$ & $-0,01$ & $-0,03533$ \\
\hline 216 & $-0,11233$ & $-0,09167$ & $-0,053$ & $-0,01$ & $-0,03667$ \\
\hline 217 & $-0,11333$ & $-0,092$ & $-0,054$ & $-0,01167$ & $-0,036$ \\
\hline 218 & $-0,113$ & $-0,09133$ & $-0,053$ & $-0,01067$ & $-0,036$ \\
\hline 219 & $-0,113$ & $-0,09133$ & $-0,05267$ & $-0,009$ & $-0,03467$ \\
\hline 220 & $-0,11467$ & $-0,092$ & $-0,055$ & $-0,011$ & $-0,03733$ \\
\hline 221 & $-0,11467$ & $-0,09267$ & $-0,05467$ & $-0,01133$ & $-0,036$ \\
\hline 222 & $-0,11467$ & $-0,09267$ & $-0,055$ & $-0,01133$ & $-0,037$ \\
\hline 223 & $-0,11467$ & $-0,09333$ & $-0,05467$ & $-0,01033$ & $-0,037$ \\
\hline 224 & $-0,116$ & $-0,09333$ & $-0,05467$ & $-0,01$ & $-0,036$ \\
\hline 225 & $-0,11633$ & $-0,095$ & $-0,055$ & $-0,01033$ & $-0,03733$ \\
\hline 226 & $-0,11667$ & $-0,09367$ & $-0,055$ & $-0,01033$ & $-0,037$ \\
\hline 227 & $-0,11633$ & $-0,09433$ & $-0,055$ & $-0,00967$ & $-0,037$ \\
\hline 228 & $-0,11733$ & $-0,09433$ & $-0,056$ & $-0,01033$ & $-0,03733$ \\
\hline 229 & $-0,11767$ & $-0,095$ & $-0,05533$ & $-0,01067$ & $-0,03733$ \\
\hline 230 & $-0,117$ & $-0,09533$ & $-0,05667$ & $-0,01033$ & $-0,03833$ \\
\hline 231 & $-0,11767$ & $-0,09567$ & $-0,056$ & $-0,01$ & $-0,03633$ \\
\hline 232 & $-0,11867$ & $-0,09533$ & $-0,05633$ & $-0,01067$ & $-0,03733$ \\
\hline 233 & $-0,11867$ & $-0,096$ & $-0,05633$ & $-0,00933$ & $-0,03767$ \\
\hline 234 & $-0,12067$ & $-0,09767$ & $-0,058$ & $-0,01133$ & $-0,039$ \\
\hline 235 & $-0,12033$ & $-0,09733$ & $-0,05733$ & $-0,011$ & $-0,03867$ \\
\hline 236 & $-0,121$ & $-0,09833$ & $-0,05733$ & $-0,01167$ & $-0,038$ \\
\hline 237 & $-0,12167$ & $-0,097$ & $-0,057$ & $-0,01133$ & $-0,03833$ \\
\hline 238 & $-0,122$ & $-0,099$ & $-0,059$ & $-0,012$ & $-0,03933$ \\
\hline 239 & $-0,12167$ & $-0,09933$ & $-0,059$ & $-0,01133$ & $-0,03933$ \\
\hline 240 & $-0,12267$ & $-0,09867$ & $-0,05867$ & $-0,01133$ & $-0,03867$ \\
\hline 241 & $-0,123$ & $-0,09933$ & $-0,05933$ & $-0,01133$ & $-0,039$ \\
\hline 242 & $-0,12267$ & $-0,099$ & $-0,059$ & $-0,01033$ & $-0,03833$ \\
\hline 243 & $-0,123$ & $-0,099$ & $-0,059$ & $-0,01033$ & $-0,03867$ \\
\hline 244 & $-0,125$ & $-0,101$ & $-0,06$ & $-0,01167$ & $-0,03967$ \\
\hline 245 & $-0,125$ & $-0,101$ & $-0,06033$ & $-0,01167$ & $-0,041$ \\
\hline 246 & $-0,12467$ & $-0,10067$ & $-0,06033$ & $-0,011$ & $-0,03967$ \\
\hline 247 & $-0,12533$ & $-0,10033$ & $-0,06133$ & $-0,01067$ & $-0,03933$ \\
\hline 248 & $-0,12533$ & $-0,1$ & $-0,06$ & $-0,01067$ & $-0,03967$ \\
\hline 249 & $-0,12633$ & $-0,101$ & $-0,06133$ & $-0,01167$ & $-0,041$ \\
\hline 250 & $-0,12833$ & $-0,103$ & $-0,062$ & $-0,013$ & $-0,042$ \\
\hline 251 & $-0,12733$ & $-0,102$ & $-0,061$ & $-0,012$ & $-0,04033$ \\
\hline 252 & $-0,12733$ & $-0,103$ & $-0,06133$ & $-0,012$ & $-0,041$ \\
\hline 253 & $-0,128$ & $-0,10267$ & $-0,06167$ & $-0,011$ & $-0,04133$ \\
\hline 254 & $-0,12867$ & $-0,10333$ & $-0,062$ & $-0,01133$ & $-0,04133$ \\
\hline 255 & $-0,129$ & $-0,10367$ & $-0,063$ & $-0,013$ & $-0,04233$ \\
\hline
\end{tabular}




\begin{tabular}{|c|c|c|c|c|c|}
\hline 256 & $-0,12833$ & $-0,10333$ & $-0,06167$ & $-0,01033$ & $-0,04067$ \\
\hline 257 & $-0,12967$ & $-0,10367$ & $-0,06233$ & $-0,01133$ & $-0,04167$ \\
\hline 258 & $-0,13067$ & $-0,10367$ & $-0,063$ & $-0,01133$ & $-0,04167$ \\
\hline 259 & $-0,131$ & $-0,10433$ & $-0,06367$ & $-0,012$ & $-0,042$ \\
\hline 260 & $-0,13133$ & $-0,105$ & $-0,06367$ & $-0,011$ & $-0,042$ \\
\hline 261 & $-0,13233$ & $-0,105$ & $-0,06433$ & $-0,01167$ & $-0,043$ \\
\hline 262 & $-0,13167$ & $-0,106$ & $-0,06433$ & $-0,013$ & $-0,04233$ \\
\hline 263 & $-0,13333$ & $-0,10533$ & $-0,064$ & $-0,012$ & $-0,04267$ \\
\hline 264 & $-0,13233$ & $-0,105$ & $-0,06333$ & $-0,011$ & $-0,04167$ \\
\hline 265 & $-0,13233$ & $-0,10633$ & $-0,06433$ & $-0,01133$ & $-0,04233$ \\
\hline 266 & $-0,13333$ & $-0,107$ & $-0,065$ & $-0,01267$ & $-0,043$ \\
\hline 267 & $-0,13333$ & $-0,10633$ & $-0,06533$ & $-0,01167$ & $-0,04267$ \\
\hline 268 & $-0,134$ & $-0,10667$ & $-0,06433$ & $-0,011$ & $-0,042$ \\
\hline 269 & $-0,13433$ & $-0,10667$ & $-0,065$ & $-0,01133$ & $-0,043$ \\
\hline 270 & $-0,134$ & $-0,10733$ & $-0,06633$ & $-0,012$ & $-0,044$ \\
\hline 271 & $-0,136$ & $-0,108$ & $-0,06667$ & $-0,01167$ & $-0,04333$ \\
\hline 272 & $-0,13567$ & $-0,10667$ & $-0,06567$ & $-0,011$ & $-0,043$ \\
\hline 273 & $-0,136$ & $-0,10733$ & $-0,066$ & $-0,01167$ & $-0,043$ \\
\hline 274 & $-0,13633$ & $-0,10833$ & $-0,066$ & $-0,01033$ & $-0,04433$ \\
\hline 275 & $-0,13667$ & $-0,10767$ & $-0,066$ & $-0,011$ & $-0,04333$ \\
\hline 276 & $-0,13733$ & $-0,10933$ & $-0,067$ & $-0,01133$ & $-0,04367$ \\
\hline 277 & $-0,13767$ & $-0,109$ & $-0,067$ & $-0,011$ & $-0,04467$ \\
\hline 278 & $-0,13833$ & $-0,10867$ & $-0,06767$ & $-0,01167$ & $-0,044$ \\
\hline 279 & $-0,138$ & $-0,109$ & $-0,06633$ & $-0,011$ & $-0,04267$ \\
\hline 280 & $-0,13867$ & $-0,10833$ & $-0,06733$ & $-0,01067$ & $-0,04433$ \\
\hline 281 & $-0,14$ & $-0,111$ & $-0,068$ & $-0,01233$ & $-0,045$ \\
\hline 282 & $-0,13933$ & $-0,10933$ & $-0,06667$ & $-0,01067$ & $-0,04467$ \\
\hline 283 & $-0,14067$ & $-0,11067$ & $-0,06833$ & $-0,01133$ & $-0,046$ \\
\hline 284 & $-0,14067$ & $-0,11133$ & $-0,06833$ & $-0,01133$ & $-0,045$ \\
\hline 285 & $-0,14233$ & $-0,11267$ & $-0,069$ & $-0,01233$ & $-0,045$ \\
\hline 286 & $-0,14167$ & $-0,11133$ & $-0,069$ & $-0,012$ & $-0,04367$ \\
\hline 287 & $-0,141$ & $-0,11033$ & $-0,069$ & $-0,01133$ & $-0,04567$ \\
\hline 288 & $-0,14233$ & $-0,112$ & $-0,06967$ & $-0,011$ & $-0,04567$ \\
\hline 289 & $-0,14333$ & $-0,11267$ & $-0,07067$ & $-0,01167$ & $-0,04567$ \\
\hline 290 & $-0,14433$ & $-0,11333$ & $-0,07033$ & $-0,01267$ & $-0,046$ \\
\hline 291 & $-0,14333$ & $-0,113$ & $-0,069$ & $-0,011$ & $-0,04533$ \\
\hline 292 & $-0,14367$ & $-0,11267$ & $-0,07$ & $-0,01133$ & $-0,04533$ \\
\hline 293 & $-0,14367$ & $-0,113$ & $-0,06967$ & $-0,011$ & $-0,04533$ \\
\hline 294 & $-0,14433$ & $-0,11333$ & $-0,071$ & $-0,012$ & $-0,04633$ \\
\hline 295 & $-0,14433$ & $-0,11333$ & $-0,07$ & $-0,01067$ & $-0,045$ \\
\hline 296 & $-0,14533$ & $-0,11533$ & $-0,072$ & $-0,012$ & $-0,04667$ \\
\hline 297 & $-0,14567$ & $-0,11367$ & $-0,07133$ & $-0,012$ & $-0,04567$ \\
\hline 298 & $-0,14567$ & $-0,11433$ & $-0,07033$ & $-0,01133$ & $-0,045$ \\
\hline 299 & $-0,14533$ & $-0,11433$ & $-0,07067$ & $-0,01$ & $-0,047$ \\
\hline 300 & $-0,14567$ & $-0,114$ & $-0,06967$ & $-0,01067$ & $-0,046$ \\
\hline
\end{tabular}




\begin{tabular}{|c|c|c|c|c|c|}
\hline 301 & $-0,146$ & $-0,11433$ & $-0,073$ & $-0,012$ & $-0,04567$ \\
\hline 302 & $-0,14667$ & $-0,11633$ & $-0,07167$ & $-0,01167$ & $-0,047$ \\
\hline 303 & $-0,148$ & $-0,11667$ & $-0,07333$ & $-0,012$ & $-0,048$ \\
\hline 304 & $-0,14833$ & $-0,11633$ & $-0,07333$ & $-0,01233$ & $-0,04633$ \\
\hline 305 & $-0,14767$ & $-0,115$ & $-0,07167$ & $-0,011$ & $-0,046$ \\
\hline 306 & $-0,14833$ & $-0,11533$ & $-0,07367$ & $-0,011$ & $-0,047$ \\
\hline 307 & $-0,14933$ & $-0,116$ & $-0,073$ & $-0,01133$ & $-0,04767$ \\
\hline 308 & $-0,14833$ & $-0,11633$ & $-0,07233$ & $-0,011$ & $-0,04733$ \\
\hline 309 & $-0,14967$ & $-0,116$ & $-0,07333$ & $-0,01133$ & $-0,047$ \\
\hline 310 & $-0,15$ & $-0,117$ & $-0,07333$ & $-0,01167$ & $-0,04667$ \\
\hline 311 & $-0,151$ & $-0,11733$ & $-0,07433$ & $-0,01233$ & $-0,04833$ \\
\hline 312 & $-0,15167$ & $-0,11867$ & $-0,076$ & $-0,01233$ & $-0,04867$ \\
\hline 313 & $-0,15133$ & $-0,11833$ & $-0,07567$ & $-0,012$ & $-0,04867$ \\
\hline 314 & $-0,152$ & $-0,119$ & $-0,074$ & $-0,012$ & $-0,04833$ \\
\hline 315 & $-0,15333$ & $-0,12033$ & $-0,07733$ & $-0,013$ & $-0,049$ \\
\hline 316 & $-0,15233$ & $-0,11833$ & $-0,07433$ & $-0,012$ & $-0,04867$ \\
\hline 317 & $-0,15367$ & $-0,12$ & $-0,078$ & $-0,01267$ & $-0,05$ \\
\hline 318 & $-0,15233$ & $-0,11867$ & $-0,076$ & $-0,011$ & $-0,04867$ \\
\hline 319 & $-0,154$ & $-0,121$ & $-0,078$ & $-0,01333$ & $-0,05$ \\
\hline 320 & $-0,15433$ & $-0,12033$ & $-0,076$ & $-0,01267$ & $-0,05$ \\
\hline 321 & $-0,154$ & $-0,12$ & $-0,076$ & $-0,012$ & $-0,04867$ \\
\hline 322 & $-0,15467$ & $-0,12167$ & $-0,07833$ & $-0,01233$ & $-0,04933$ \\
\hline 323 & $-0,15533$ & $-0,12133$ & $-0,07867$ & $-0,01233$ & $-0,05033$ \\
\hline 324 & $-0,155$ & $-0,121$ & $-0,07667$ & $-0,01167$ & $-0,04967$ \\
\hline 325 & $-0,155$ & $-0,12067$ & $-0,07767$ & $-0,01133$ & $-0,049$ \\
\hline 326 & $-0,15667$ & $-0,121$ & $-0,078$ & $-0,012$ & $-0,05$ \\
\hline 327 & $-0,156$ & $-0,12167$ & $-0,07833$ & $-0,01267$ & $-0,05$ \\
\hline 328 & $-0,15733$ & $-0,12267$ & $-0,077$ & $-0,01233$ & $-0,05$ \\
\hline 329 & $-0,157$ & $-0,12167$ & $-0,07967$ & $-0,01233$ & $-0,04967$ \\
\hline 330 & $-0,15733$ & $-0,12267$ & $-0,07967$ & $-0,01267$ & $-0,05067$ \\
\hline 331 & $-0,15733$ & $-0,12267$ & $-0,07933$ & $-0,01233$ & $-0,05033$ \\
\hline 332 & $-0,15767$ & $-0,12267$ & $-0,07867$ & $-0,01167$ & $-0,05067$ \\
\hline 333 & $-0,158$ & $-0,122$ & $-0,08$ & $-0,01133$ & $-0,05133$ \\
\hline 334 & $-0,15833$ & $-0,12467$ & $-0,08$ & $-0,01233$ & $-0,05033$ \\
\hline 335 & $-0,15867$ & $-0,12367$ & $-0,07867$ & $-0,01167$ & $-0,051$ \\
\hline 336 & $-0,15933$ & $-0,124$ & $-0,08133$ & $-0,01233$ & $-0,05167$ \\
\hline 337 & $-0,16067$ & $-0,12467$ & $-0,08067$ & $-0,01267$ & $-0,052$ \\
\hline 338 & $-0,15933$ & $-0,12333$ & $-0,07867$ & $-0,01167$ & $-0,05033$ \\
\hline 339 & $-0,15933$ & $-0,124$ & $-0,08$ & $-0,012$ & $-0,05133$ \\
\hline 340 & $-0,16067$ & $-0,12433$ & $-0,081$ & $-0,011$ & $-0,05067$ \\
\hline 341 & $-0,16167$ & $-0,12467$ & $-0,08$ & $-0,01267$ & $-0,05167$ \\
\hline 342 & $-0,16133$ & $-0,12433$ & $-0,07967$ & $-0,012$ & $-0,051$ \\
\hline 343 & $-0,16167$ & $-0,12467$ & $-0,081$ & $-0,012$ & $-0,05167$ \\
\hline 344 & $-0,163$ & $-0,12633$ & $-0,08333$ & $-0,01333$ & $-0,052$ \\
\hline 345 & $-0,161$ & $-0,125$ & $-0,07967$ & $-0,01133$ & $-0,05133$ \\
\hline
\end{tabular}




\begin{tabular}{|c|c|c|c|c|c|}
\hline 346 & $-0,16367$ & $-0,12733$ & $-0,084$ & $-0,013$ & $-0,05333$ \\
\hline 347 & $-0,162$ & $-0,12567$ & $-0,08133$ & $-0,01167$ & $-0,053$ \\
\hline 348 & $-0,16233$ & $-0,125$ & $-0,08067$ & $-0,01067$ & $-0,05133$ \\
\hline 349 & $-0,164$ & $-0,12733$ & $-0,08433$ & $-0,01233$ & $-0,05333$ \\
\hline 350 & $-0,16367$ & $-0,12633$ & $-0,08267$ & $-0,012$ & $-0,05233$ \\
\hline 351 & $-0,16367$ & $-0,12733$ & $-0,08233$ & $-0,013$ & $-0,05233$ \\
\hline 352 & $-0,16433$ & $-0,127$ & $-0,083$ & $-0,012$ & $-0,052$ \\
\hline 353 & $-0,166$ & $-0,12733$ & $-0,08267$ & $-0,013$ & $-0,053$ \\
\hline 354 & $-0,16633$ & $-0,12933$ & $-0,084$ & $-0,01333$ & $-0,05467$ \\
\hline 355 & $-0,16533$ & $-0,12767$ & $-0,08533$ & $-0,01333$ & $-0,054$ \\
\hline 356 & $-0,167$ & $-0,12767$ & $-0,08433$ & $-0,012$ & $-0,054$ \\
\hline 357 & $-0,166$ & $-0,12833$ & $-0,08467$ & $-0,01233$ & $-0,05367$ \\
\hline 358 & $-0,16667$ & $-0,129$ & $-0,08433$ & $-0,01267$ & $-0,05367$ \\
\hline 359 & $-0,16667$ & $-0,12867$ & $-0,08433$ & $-0,01233$ & $-0,05367$ \\
\hline 360 & $-0,16767$ & $-0,129$ & $-0,08433$ & $-0,01267$ & $-0,054$ \\
\hline 361 & $-0,16933$ & $-0,13$ & $-0,085$ & $-0,01333$ & $-0,05433$ \\
\hline 362 & $-0,168$ & $-0,12833$ & $-0,084$ & $-0,01167$ & $-0,053$ \\
\hline 363 & $-0,169$ & $-0,13$ & $-0,086$ & $-0,01233$ & $-0,05467$ \\
\hline 364 & $-0,16867$ & $-0,12933$ & $-0,085$ & $-0,01233$ & $-0,05467$ \\
\hline 365 & $-0,16967$ & $-0,12867$ & $-0,08533$ & $-0,01233$ & $-0,05333$ \\
\hline 366 & $-0,16933$ & $-0,13033$ & $-0,08533$ & $-0,01233$ & $-0,05433$ \\
\hline 367 & $-0,17133$ & $-0,13167$ & $-0,08733$ & $-0,014$ & $-0,05633$ \\
\hline 368 & $-0,17067$ & $-0,13$ & $-0,086$ & $-0,01267$ & $-0,055$ \\
\hline 369 & $-0,17133$ & $-0,13133$ & $-0,08667$ & $-0,013$ & $-0,05567$ \\
\hline 370 & $-0,17033$ & $-0,12967$ & $-0,086$ & $-0,01133$ & $-0,05433$ \\
\hline 371 & $-0,171$ & $-0,13033$ & $-0,08633$ & $-0,01233$ & $-0,05433$ \\
\hline 372 & $-0,17067$ & $-0,131$ & $-0,08667$ & $-0,01267$ & $-0,05533$ \\
\hline 373 & $-0,173$ & $-0,132$ & $-0,08733$ & $-0,01267$ & $-0,05567$ \\
\hline 374 & $-0,17267$ & $-0,132$ & $-0,08733$ & $-0,01267$ & $-0,05467$ \\
\hline 375 & $-0,172$ & $-0,13133$ & $-0,088$ & $-0,01233$ & $-0,05467$ \\
\hline 376 & $-0,173$ & $-0,13167$ & $-0,08833$ & $-0,01333$ & $-0,05567$ \\
\hline 377 & $-0,17367$ & $-0,132$ & $-0,08733$ & $-0,01233$ & $-0,05567$ \\
\hline 378 & $-0,17367$ & $-0,13267$ & $-0,08867$ & $-0,01233$ & $-0,05567$ \\
\hline 379 & $-0,175$ & $-0,13233$ & $-0,089$ & $-0,013$ & $-0,05633$ \\
\hline 380 & $-0,17567$ & $-0,13367$ & $-0,08967$ & $-0,01333$ & $-0,05667$ \\
\hline 381 & $-0,175$ & $-0,13367$ & $-0,08933$ & $-0,013$ & $-0,05733$ \\
\hline 382 & $-0,176$ & $-0,13433$ & $-0,09$ & $-0,01367$ & $-0,05733$ \\
\hline 383 & $-0,17567$ & $-0,13367$ & $-0,08967$ & $-0,013$ & $-0,057$ \\
\hline 384 & $-0,17633$ & $-0,134$ & $-0,089$ & $-0,01267$ & $-0,057$ \\
\hline 385 & $-0,177$ & $-0,13533$ & $-0,09$ & $-0,01367$ & $-0,05667$ \\
\hline 386 & $-0,177$ & $-0,13367$ & $-0,09$ & $-0,01233$ & $-0,05767$ \\
\hline 387 & $-0,17767$ & $-0,13467$ & $-0,08967$ & $-0,013$ & $-0,057$ \\
\hline 388 & $-0,177$ & $-0,13467$ & $-0,08933$ & $-0,01233$ & $-0,05667$ \\
\hline 389 & $-0,178$ & $-0,135$ & $-0,09033$ & $-0,01367$ & $-0,05667$ \\
\hline 390 & $-0,17867$ & $-0,13533$ & $-0,09133$ & $-0,013$ & $-0,05733$ \\
\hline
\end{tabular}




\begin{tabular}{|c|c|c|c|c|c|}
\hline 391 & $-0,17867$ & $-0,137$ & $-0,09167$ & $-0,014$ & $-0,058$ \\
\hline 392 & $-0,17833$ & $-0,135$ & $-0,091$ & $-0,01333$ & $-0,05833$ \\
\hline 393 & $-0,18033$ & $-0,136$ & $-0,092$ & $-0,01333$ & $-0,058$ \\
\hline 394 & $-0,17967$ & $-0,13567$ & $-0,09067$ & $-0,01333$ & $-0,057$ \\
\hline 395 & $-0,17867$ & $-0,13567$ & $-0,09167$ & $-0,013$ & $-0,05767$ \\
\hline 396 & $-0,17933$ & $-0,136$ & $-0,092$ & $-0,013$ & $-0,05833$ \\
\hline 397 & $-0,18067$ & $-0,13567$ & $-0,092$ & $-0,01367$ & $-0,05833$ \\
\hline 398 & $-0,18133$ & $-0,137$ & $-0,09133$ & $-0,01233$ & $-0,05733$ \\
\hline 399 & $-0,17967$ & $-0,136$ & $-0,09167$ & $-0,01233$ & $-0,05733$ \\
\hline 400 & $-0,18233$ & $-0,137$ & $-0,09233$ & $-0,013$ & $-0,059$ \\
\hline 401 & $-0,182$ & $-0,13767$ & $-0,09333$ & $-0,01433$ & $-0,058$ \\
\hline 402 & $-0,18267$ & $-0,13767$ & $-0,094$ & $-0,01367$ & $-0,059$ \\
\hline 403 & $-0,18233$ & $-0,13767$ & $-0,094$ & $-0,014$ & $-0,05933$ \\
\hline 404 & $-0,182$ & $-0,13833$ & $-0,09333$ & $-0,01233$ & $-0,059$ \\
\hline 405 & $-0,18467$ & $-0,14$ & $-0,095$ & $-0,01533$ & $-0,05967$ \\
\hline 406 & $-0,18433$ & $-0,13833$ & $-0,09433$ & $-0,01367$ & $-0,05967$ \\
\hline 407 & $-0,184$ & $-0,13933$ & $-0,09467$ & $-0,01467$ & $-0,05967$ \\
\hline 408 & $-0,186$ & $-0,13967$ & $-0,096$ & $-0,015$ & $-0,06033$ \\
\hline 409 & $-0,18467$ & $-0,13833$ & $-0,09467$ & $-0,01333$ & $-0,059$ \\
\hline 410 & $-0,18567$ & $-0,14033$ & $-0,096$ & $-0,014$ & $-0,06$ \\
\hline 411 & $-0,18433$ & $-0,138$ & $-0,094$ & $-0,012$ & $-0,059$ \\
\hline 412 & $-0,186$ & $-0,13933$ & $-0,095$ & $-0,01267$ & $-0,05933$ \\
\hline 413 & $-0,18667$ & $-0,14033$ & $-0,09633$ & $-0,01467$ & $-0,06033$ \\
\hline 414 & $-0,18667$ & $-0,14033$ & $-0,09533$ & $-0,01333$ & $-0,06$ \\
\hline 415 & $-0,18833$ & $-0,14167$ & $-0,098$ & $-0,01467$ & $-0,062$ \\
\hline 416 & $-0,18733$ & $-0,13967$ & $-0,09633$ & $-0,015$ & $-0,06$ \\
\hline 417 & $-0,188$ & $-0,141$ & $-0,097$ & $-0,01467$ & $-0,06167$ \\
\hline 418 & $-0,189$ & $-0,14133$ & $-0,098$ & $-0,01433$ & $-0,06133$ \\
\hline 419 & $-0,18933$ & $-0,14267$ & $-0,09767$ & $-0,015$ & $-0,06167$ \\
\hline 420 & $-0,18933$ & $-0,14167$ & $-0,098$ & $-0,01367$ & $-0,06133$ \\
\hline 421 & $-0,189$ & $-0,14233$ & $-0,09767$ & $-0,015$ & $-0,061$ \\
\hline 422 & $-0,18933$ & $-0,143$ & $-0,09733$ & $-0,015$ & $-0,061$ \\
\hline 423 & $-0,18867$ & $-0,14133$ & $-0,09767$ & $-0,01367$ & $-0,06067$ \\
\hline 424 & $-0,18967$ & $-0,14167$ & $-0,09833$ & $-0,01333$ & $-0,06167$ \\
\hline 425 & $-0,191$ & $-0,143$ & $-0,09933$ & $-0,01433$ & $-0,062$ \\
\hline 426 & $-0,19167$ & $-0,143$ & $-0,09933$ & $-0,01533$ & $-0,06167$ \\
\hline 427 & $-0,19167$ & $-0,143$ & $-0,09867$ & $-0,014$ & $-0,06133$ \\
\hline 428 & $-0,19233$ & $-0,14333$ & $-0,09967$ & $-0,015$ & $-0,062$ \\
\hline 429 & $-0,19167$ & $-0,142$ & $-0,09867$ & $-0,01333$ & $-0,06033$ \\
\hline 430 & $-0,193$ & $-0,14467$ & $-0,10033$ & $-0,015$ & $-0,06333$ \\
\hline 431 & $-0,193$ & $-0,144$ & $-0,10033$ & $-0,01467$ & $-0,062$ \\
\hline 432 & $-0,19233$ & $-0,14367$ & $-0,09967$ & $-0,01433$ & $-0,06233$ \\
\hline 433 & $-0,194$ & $-0,14267$ & $-0,09933$ & $-0,01433$ & $-0,06167$ \\
\hline 434 & $-0,19333$ & $-0,14367$ & $-0,10033$ & $-0,015$ & $-0,06267$ \\
\hline 435 & $-0,19467$ & $-0,14433$ & $-0,10033$ & $-0,01433$ & $-0,06333$ \\
\hline
\end{tabular}




\begin{tabular}{|c|c|c|c|c|c|}
\hline 436 & $-0,194$ & $-0,14433$ & $-0,101$ & $-0,01533$ & $-0,06167$ \\
\hline 437 & $-0,19333$ & $-0,144$ & $-0,10067$ & $-0,01433$ & $-0,06233$ \\
\hline 438 & $-0,194$ & $-0,144$ & $-0,10067$ & $-0,01433$ & $-0,06233$ \\
\hline 439 & $-0,19533$ & $-0,14467$ & $-0,10133$ & $-0,015$ & $-0,062$ \\
\hline 440 & $-0,19667$ & $-0,14633$ & $-0,10333$ & $-0,01533$ & $-0,06367$ \\
\hline 441 & $-0,19533$ & $-0,145$ & $-0,10133$ & $-0,014$ & $-0,06233$ \\
\hline 442 & $-0,19667$ & $-0,145$ & $-0,10267$ & $-0,01467$ & $-0,06367$ \\
\hline 443 & $-0,19733$ & $-0,146$ & $-0,10267$ & $-0,01433$ & $-0,06333$ \\
\hline 444 & $-0,19633$ & $-0,14567$ & $-0,10167$ & $-0,01433$ & $-0,06267$ \\
\hline 445 & $-0,197$ & $-0,14633$ & $-0,10267$ & $-0,01433$ & $-0,063$ \\
\hline 446 & $-0,197$ & $-0,14533$ & $-0,10233$ & $-0,014$ & $-0,063$ \\
\hline 447 & $-0,19767$ & $-0,14633$ & $-0,10267$ & $-0,01533$ & $-0,06433$ \\
\hline 448 & $-0,198$ & $-0,14633$ & $-0,10433$ & $-0,015$ & $-0,064$ \\
\hline 449 & $-0,199$ & $-0,14633$ & $-0,10333$ & $-0,014$ & $-0,065$ \\
\hline 450 & $-0,19867$ & $-0,14633$ & $-0,10233$ & $-0,01433$ & $-0,064$ \\
\hline 451 & $-0,19833$ & $-0,14567$ & $-0,104$ & $-0,014$ & $-0,06333$ \\
\hline 452 & $-0,19967$ & $-0,14733$ & $-0,10367$ & $-0,015$ & $-0,06467$ \\
\hline 453 & $-0,19967$ & $-0,14733$ & $-0,10467$ & $-0,015$ & $-0,064$ \\
\hline 454 & $-0,20033$ & $-0,148$ & $-0,10467$ & $-0,01467$ & $-0,06433$ \\
\hline 455 & $-0,20067$ & $-0,148$ & $-0,10533$ & $-0,015$ & $-0,06433$ \\
\hline 456 & $-0,20067$ & $-0,14733$ & $-0,10367$ & $-0,01467$ & $-0,064$ \\
\hline 457 & $-0,201$ & $-0,14767$ & $-0,10433$ & $-0,01533$ & $-0,064$ \\
\hline 458 & $-0,20133$ & $-0,14833$ & $-0,10567$ & $-0,01533$ & $-0,06533$ \\
\hline 459 & $-0,20133$ & $-0,14833$ & $-0,10533$ & $-0,015$ & $-0,066$ \\
\hline 460 & $-0,202$ & $-0,14833$ & $-0,10633$ & $-0,01533$ & $-0,066$ \\
\hline 461 & $-0,20267$ & $-0,14933$ & $-0,10667$ & $-0,01633$ & $-0,066$ \\
\hline 462 & $-0,20367$ & $-0,149$ & $-0,10567$ & $-0,01533$ & $-0,066$ \\
\hline 463 & $-0,20367$ & $-0,14967$ & $-0,10667$ & $-0,01667$ & $-0,06633$ \\
\hline 464 & $-0,203$ & $-0,14867$ & $-0,10633$ & $-0,015$ & $-0,06633$ \\
\hline 465 & $-0,204$ & $-0,14967$ & $-0,10767$ & $-0,01567$ & $-0,066$ \\
\hline 466 & $-0,20467$ & $-0,15033$ & $-0,10733$ & $-0,01667$ & $-0,06567$ \\
\hline 467 & $-0,20333$ & $-0,14833$ & $-0,10667$ & $-0,01467$ & $-0,06567$ \\
\hline 468 & $-0,205$ & $-0,15$ & $-0,10733$ & $-0,01567$ & $-0,06667$ \\
\hline 469 & $-0,20567$ & $-0,151$ & $-0,10833$ & $-0,01633$ & $-0,06767$ \\
\hline 470 & $-0,20567$ & $-0,15067$ & $-0,107$ & $-0,01533$ & $-0,06667$ \\
\hline 471 & $-0,20633$ & $-0,15033$ & $-0,10833$ & $-0,01567$ & $-0,06667$ \\
\hline 472 & $-0,20567$ & $-0,15033$ & $-0,10767$ & $-0,015$ & $-0,06667$ \\
\hline 473 & $-0,206$ & $-0,15067$ & $-0,10833$ & $-0,01467$ & $-0,066$ \\
\hline 474 & $-0,20667$ & $-0,151$ & $-0,10833$ & $-0,01567$ & $-0,06633$ \\
\hline 475 & $-0,20667$ & $-0,151$ & $-0,10833$ & $-0,01467$ & $-0,06667$ \\
\hline 476 & $-0,20633$ & $-0,151$ & $-0,10867$ & $-0,01467$ & $-0,066$ \\
\hline 477 & $-0,20767$ & $-0,15167$ & $-0,10967$ & $-0,01533$ & $-0,06633$ \\
\hline 478 & $-0,20833$ & $-0,15167$ & $-0,10967$ & $-0,01567$ & $-0,06667$ \\
\hline 479 & $-0,20933$ & $-0,15233$ & $-0,11067$ & $-0,017$ & $-0,06767$ \\
\hline 480 & $-0,209$ & $-0,15133$ & $-0,11033$ & $-0,01533$ & $-0,068$ \\
\hline
\end{tabular}




\begin{tabular}{|c|c|c|c|c|c|}
\hline 481 & $-0,21$ & $-0,153$ & $-0,11067$ & $-0,01633$ & $-0,06767$ \\
\hline 482 & $-0,209$ & $-0,152$ & $-0,111$ & $-0,01567$ & $-0,06767$ \\
\hline 483 & $-0,21$ & $-0,15267$ & $-0,111$ & $-0,01567$ & $-0,06733$ \\
\hline 484 & $-0,21033$ & $-0,15167$ & $-0,11067$ & $-0,01667$ & $-0,06833$ \\
\hline 485 & $-0,21267$ & $-0,15367$ & $-0,11133$ & $-0,01733$ & $-0,06867$ \\
\hline 486 & $-0,20967$ & $-0,15167$ & $-0,11067$ & $-0,015$ & $-0,067$ \\
\hline 487 & $-0,21$ & $-0,15233$ & $-0,111$ & $-0,01567$ & $-0,06733$ \\
\hline 488 & $-0,21233$ & $-0,15367$ & $-0,11133$ & $-0,01733$ & $-0,06867$ \\
\hline 489 & $-0,212$ & $-0,15367$ & $-0,11233$ & $-0,01633$ & $-0,069$ \\
\hline 490 & $-0,21267$ & $-0,15333$ & $-0,11133$ & $-0,01667$ & $-0,069$ \\
\hline 491 & $-0,212$ & $-0,15267$ & $-0,112$ & $-0,016$ & $-0,06933$ \\
\hline 492 & $-0,21333$ & $-0,15433$ & $-0,11267$ & $-0,01667$ & $-0,06967$ \\
\hline 493 & $-0,214$ & $-0,15433$ & $-0,11433$ & $-0,017$ & $-0,071$ \\
\hline 494 & $-0,21333$ & $-0,15533$ & $-0,11333$ & $-0,01667$ & $-0,069$ \\
\hline 495 & $-0,214$ & $-0,15467$ & $-0,11367$ & $-0,01633$ & $-0,069$ \\
\hline 496 & $-0,21467$ & $-0,155$ & $-0,114$ & $-0,01767$ & $-0,07$ \\
\hline 497 & $-0,21467$ & $-0,15533$ & $-0,11433$ & $-0,017$ & $-0,06933$ \\
\hline 498 & $-0,21633$ & $-0,15567$ & $-0,11433$ & $-0,01767$ & $-0,07$ \\
\hline 499 & $-0,21433$ & $-0,154$ & $-0,11367$ & $-0,016$ & $-0,06933$ \\
\hline 500 & $-0,216$ & $-0,15567$ & $-0,11367$ & $-0,017$ & $-0,07067$ \\
\hline 501 & $-0,21567$ & $-0,15567$ & $-0,11367$ & $-0,01667$ & $-0,07$ \\
\hline 502 & $-0,21567$ & $-0,15467$ & $-0,11367$ & $-0,01567$ & $-0,06967$ \\
\hline 503 & $-0,21567$ & $-0,15433$ & $-0,114$ & $-0,01467$ & $-0,06967$ \\
\hline 504 & $-0,216$ & $-0,15533$ & $-0,114$ & $-0,016$ & $-0,07$ \\
\hline 505 & $-0,217$ & $-0,15567$ & $-0,115$ & $-0,01633$ & $-0,07067$ \\
\hline 506 & $-0,21767$ & $-0,15667$ & $-0,11533$ & $-0,01667$ & $-0,07033$ \\
\hline 507 & $-0,21767$ & $-0,156$ & $-0,11567$ & $-0,01767$ & $-0,07033$ \\
\hline 508 & $-0,21733$ & $-0,155$ & $-0,115$ & $-0,01567$ & $-0,06933$ \\
\hline 509 & $-0,21833$ & $-0,15667$ & $-0,116$ & $-0,01633$ & $-0,07133$ \\
\hline 510 & $-0,21867$ & $-0,15767$ & $-0,11633$ & $-0,016$ & $-0,07133$ \\
\hline 511 & $-0,219$ & $-0,15733$ & $-0,117$ & $-0,01667$ & $-0,071$ \\
\hline 512 & $-0,21967$ & $-0,15833$ & $-0,11767$ & $-0,01733$ & $-0,07133$ \\
\hline 513 & $-0,21967$ & $-0,157$ & $-0,11733$ & $-0,01767$ & $-0,072$ \\
\hline 514 & $-0,22033$ & $-0,15767$ & $-0,117$ & $-0,01833$ & $-0,071$ \\
\hline 515 & $-0,221$ & $-0,15733$ & $-0,11767$ & $-0,01767$ & $-0,07233$ \\
\hline 516 & $-0,22133$ & $-0,15833$ & $-0,11767$ & $-0,01767$ & $-0,07233$ \\
\hline 517 & $-0,22033$ & $-0,15767$ & $-0,117$ & $-0,01667$ & $-0,07133$ \\
\hline 518 & $-0,221$ & $-0,15867$ & $-0,11767$ & $-0,01733$ & $-0,07133$ \\
\hline 519 & $-0,22133$ & $-0,158$ & $-0,11767$ & $-0,017$ & $-0,072$ \\
\hline 520 & $-0,221$ & $-0,15867$ & $-0,11833$ & $-0,017$ & $-0,07267$ \\
\hline & -- & -- & -- & & \\
\hline
\end{tabular}

